\title{
Plasma-enhanced Chemical Vapor Deposition OF SILICON DIOXIDE
}

Optimizing dielectric films through plasma characterization

Arjen Boogaard 
Samenstelling promotiecommissie:

\begin{tabular}{|c|c|c|}
\hline Voorzitter: & prof. dr. ir. A.J. Mouthaan & Universiteit Twente \\
\hline Secretaris: & prof. dr. ir. A.J. Mouthaan & \\
\hline Promotor: & prof. dr. ir. R.A.M. Wolters & Universiteit Twente \\
\hline $\begin{array}{l}\text { Assistent } \\
\text { promotor: }\end{array}$ & dr. A.Y. Kovalgin & Universiteit Twente \\
\hline Referenten: & dr. J. Holleman & Universiteit Twente \\
\hline & dr. ir. J.H. Klootwijk & Philips Research \\
\hline Leden: & prof. dr. ing. A.J.H.M. Rijnders & Universiteit Twente \\
\hline & prof. dr. J. Schmitz & Universiteit Twente \\
\hline & prof. dr. C.I.M. Beenakker & $\begin{array}{l}\text { Technische Universiteit } \\
\text { Delft }\end{array}$ \\
\hline & prof. dr. hab. inż. B. Majkusiak & Politechnika Warszawska \\
\hline & prof. dr. ir. M.C.M. van de Sanden & $\begin{array}{l}\text { Technische Universiteit } \\
\text { Eindhoven }\end{array}$ \\
\hline
\end{tabular}

Title: Plasma-enhanced chemical vapor deposition of silicon dioxide: optimizing dielectric films through plasma characterization Author: Arjen Boogaard ISBN: 978-90-365-3130-6 DOI: $10.3990 / 1.9789036531306$

Foto omslag: toegangshek in de vorm van een computerchip van de Oranjekerk in Amsterdam.

Ontwerp omslag en binnenwerk: Jos Smeets, Deventer (www.quixote.nl) Figuren binnenwerk: Ihor Brunets, Arjen Boogaard

The research was supported by the Dutch Technology Foundation STW (project "Low temperature semiconductor device fabrication" TEL.6358) and carried out in the Semiconductor Components group, MESA+ Institute for Nanotechnology, University of Twente, The Netherlands. 


\title{
Plasma-enhanced Chemical Vapor Deposition OF SILICON DIOXIDE
}

Optimizing dielectric films through plasma characterization

\author{
Arjen Boogaard
}

\section{PROEFSCHRIFT}

ter verkrijging van de graad van doctor aan de Universiteit Twente,

op gezag van de Rector Magnificus, prof. dr. H. Brinksma,

volgens besluit van het College voor Promoties

in het openbaar te verdedigen

op woensdag 12 januari 2011 om 16:45 uur

door

Arjen Boogaard

geboren op 10 september 1971

te Valkenisse 
Dit proefschrift is goedgekeurd door: prof. dr. ir. R.A.M. Wolters (promotor) dr. A.Y. Kovalgin (assistent promotor)

Copyright (c) 2011 by Arjen Boogaard, Nijmegen, the Netherlands All rights reserved. No part of this publication may be reproduced, stored in a retrieval system, or transmitted, in any form or by any means, electronic, mechanical, photocopying, recording or otherwise, in whole or in part without the prior written permission of the copyright owner. 


\section{Contents}

$\begin{array}{ll}\text { List of symbols and acronyms ix } & \text { ix }\end{array}$

1 Introduction $\quad \mathbf{1}$

1.1 Motivation and scientific challenges . . . . . . . . . . . . . . . 1

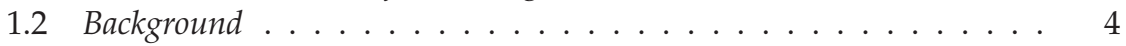

1.2.1 Developments in plasma sources . . . . . . . . . . 5

1.2 .2 PECVD ...................... 7

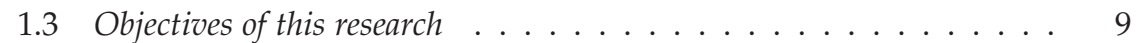

1.4 Outline of the thesis ...................... 9

I THE PLASMA STATE 11

2 Introduction $\quad 13$

3 Aspects of plasma physics 15

3.1 General ........................... 15

3.2 Particle collisions . . . . . . . . . . . . . . . . . 20

3.3 Evaluation of criteria and domains . . . . . . . . . . . . . . . . . 22

3.4 Energy transfer . . . . . . . . . . . . . . . . . . 24

4 Experimental equipment 29

4.1 ICPECVD system . . . . . . . . . . . . . . . . . . . . . . . 29

4.1.1 The variable axial magnetic field . . . . . . . . . . . . . . 31

4.1 .2 The magnetic multipole wall . . . . . . . . . . . 31

4.1.3 Impedance matching networks . . . . . . . . . . . . 32

4.1.4 External oscillator . . . . . . . . . . . . . . 33

4.1.5 Typical plasma operation conditions . . . . . . . . . . . . 35

4.1 .6 Wafer temperature control ............. 35

4.1 .7 Radiation . . . . . . . . . . . . . . . 36 
4.1 .8 Gas conduction . . . . . . . . . . . . . . . . 37

4.1 .9 Heat fluxes from plasma . . . . . . . . . . . . . . . 38

4.1.10 Heat generation due to chemical reactions . . . . . . . 39

4.2 Langmuir probe . . . . . . . . . . . . . . . . . . . . . . . 40

4.3 Optical Emission Spectroscopy (OES) . . . . . . . . . . . . . 45

4.3.1 Corona versus saha equilibrium . . . . . . . . . 46

4.3.2 Line intensities from ground states in corona equilibrium 47

4.3.3 Excitation from non-ground states . . . . . . . . . . . 50

4.3 .4 Line ratios . . . . . . . . . . . . . . . 52

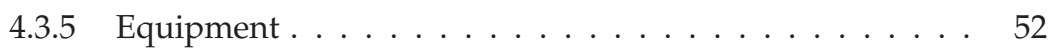

5 Experimental characterization of the discharge 55

5.1 Introduction . . . . . . . . . . . . . . . . . . . . . 55

5.2 Argon plasmas ..................... 56

5.2.1 First verification of LP measurements . . . . . . . . . . 61

5.2.2 The variable axial magnetic field . . . . . . . . . . 61

5.2.3 Second verification of the LP measurements . . . . . . . 64

5.2.4 Estimate of the density of metastable atoms . . . . . . . 69

$5.3 \mathrm{Ar}-\mathrm{N}_{2}, \mathrm{Ar}-\mathrm{N}_{2} \mathrm{O}$ and $\mathrm{Ar}-\mathrm{SiH}_{4}$ plasmas . . . . . . . . . . . 69

5.4 Conclusions . . . . . . . . . . . . . . . . . . . 70

6 Utilization of the measured plasma parameters 75

6.1 Introduction . . . . . . . . . . . . . . . . . . . . . . 75

6.2 Modeling the plasma composition . . . . . . . . . . . . 75

6.2.1 Reactions involving $\mathrm{SiH}_{4}$ and $\mathrm{H}_{2} \ldots \ldots \ldots \ldots . \ldots 76$

6.2.2 Reactions between $\mathrm{SiH}_{4}$ and $\mathrm{Ar} \ldots \ldots \ldots$. . . . . 77

6.2.3 Reactions involving $\mathrm{N}_{2} \mathrm{O}, \mathrm{N}_{2}, \mathrm{O}_{2}$, and $\mathrm{Ar} \ldots \ldots . .77$

6.2.4 Reactions involving $\mathrm{SiH}_{4}$, and $\mathrm{N}_{2} \mathrm{O} \ldots \ldots \ldots \ldots$

6.3 Typical modeling conditions . . . . . . . . . . . . . 78

6.4 Impact of EEDF on silane-based plasmas . . . . . . . . . . 78

6.5 Predicting film properties by modeling . . . . . . . . . . . . . 81

6.5 .1 Silane fraction of $0.8 \% \ldots \ldots \ldots \ldots$. . . . . . . 84

6.5 .2 Silane fraction of $0.08 \% \ldots \ldots \ldots \ldots$

6.6 Conclusions . . . . . . . . . . . . . . . . . 85 
II THE SOLID STATE $\quad 87$

$\begin{array}{llr}7 & \text { Introduction } & 89\end{array}$

8 Experimental 93

8.1 Introduction . . . . . . . . . . . . . . . . . . . . . 93

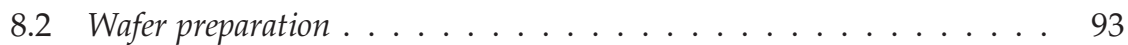

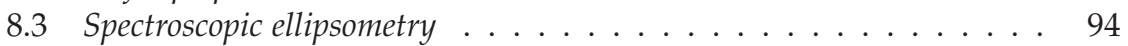

8.4 Electrical characterization . . . . . . . . . . . . . 95

8.4.1 Capacitance-voltage $(C-V)$ characteristics . . . . . . . 95

8.4.2 Effect of oxide fixed charge and interface traps . . . . . 97

8.4.3 Current-voltage $(I-V)$ characteristics . . . . . . . . 98

9 Characterization of the film properties 101

9.1 Deposition rate and material density . . . . . . . . . . . . . . . 101

9.1.1 Silane fraction of $0.8 \% \ldots \ldots \ldots 10 \ldots$

9.1 .2 Silane fraction of $0.08 \% \ldots \ldots \ldots 2$

9.2 Leakage current . . . . . . . . . . . . . . . . . . . . . . . . . . . . 102

9.2.1 Silane fraction of $0.8 \% \ldots \ldots \ldots . \ldots . \ldots 102$

9.2.2 Silane fraction of $0.08 \% \ldots \ldots \ldots$. . . . . . . . . 104

9.3 Capacitance-voltage characteristics . . . . . . . . . . . . . . . . . 106

9.3.1 Silane fraction of $0.8 \% \ldots \ldots \ldots \ldots$

9.3.2 Silane fraction of $0.08 \% \ldots \ldots$. . . . . . . . . 108

9.4 Summarizing the correlations between predicted and measured film

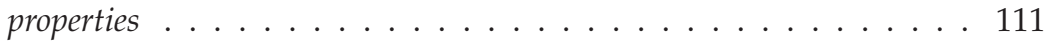

9.5 Utilization in electronic devices . . . . . . . . . . . . . . . . . . . . 111

9.6 Conclusions . . . . . . . . . . . . . . . . . . . . . 114

10 Net negative charge in $\mathrm{SiO}_{2}$ dielectric layers $\quad 117$

10.1 Introduction . . . . . . . . . . . . . . . . . . . . . . . 117

10.2 Results and discussion . . . . . . . . . . . . . . . . . . . . . . . 118

10.2.1 The electronic nature of the negative charge . . . . . . . . 124

10.2.2 The physical nature of the negative charge . . . . . . . . . 124

10.2.3 C $-V$ measurements of $\mathrm{SiO}_{2}$ :O layers . . . . . . . . . . . . 127

10.2.4 Preliminary conclusions and recommendations . . . . . 131

10.3 Conclusions . . . . . . . . . . . . . . . . . . 131

11 Conclusions and recommendations 133

11.1 Conclusions . . . . . . . . . . . . . . . . . . . . . . . . . . . . . . . . . . . . .

11.2 Recommendations for further research . . . . . . . . . . . . . . . 135 
Summary

Samenvatting

Author biography

List of publications

US patents

A Calculation of line intensity ratios

B Line intensities caused by Ar 1s 5 -metastables

Dankwoord 


\section{List of symbols and acronyms}

\begin{tabular}{|c|c|c|}
\hline Symbol & Description & (value) [unit] \\
\hline$A$ & $\begin{array}{l}\text { spontaneous emission rate or Ein- } \\
\text { stein A coefficient }\end{array}$ & {$\left[\mathrm{s}^{-1}\right]$} \\
\hline$B$ & magnetic flux density & [T] \\
\hline$C_{\mathrm{ox}}$ & oxide capacitance & {$\left[\mathrm{F} / \mathrm{cm}^{2}\right]$} \\
\hline$C_{\mathrm{s}}$ & semiconductor capacitance & {$\left[\mathrm{F} / \mathrm{cm}^{2}\right]$} \\
\hline$D_{\mathrm{a}}$ & ambipolar diffusion coefficient & {$\left[\mathrm{m}^{2} / \mathrm{s}\right]$} \\
\hline$D_{\mathrm{e}, \mathrm{i}}$ & $\begin{array}{l}\text { diffusion constant of the electrons } \\
\text { or ions }\end{array}$ & {$\left[\mathrm{m}^{2} / \mathrm{s}\right]$} \\
\hline$D_{\text {it }}$ & Interface trap density & [states $\cdot \mathrm{eV}^{-1} \mathrm{~cm}^{-2}$ ] \\
\hline$E_{\mathrm{F}}$ & Fermi energy or Fermi level & {$[\mathrm{eV}]$} \\
\hline$E_{\mathrm{Ox}}$ & oxide electric field & {$[\mathrm{V} / \mathrm{m}]$} \\
\hline$f(\theta)$ & $\begin{array}{l}\text { electrical activation fraction of im- } \\
\text { plantation dose }\end{array}$ & \\
\hline$f_{v, \mathrm{e}}$ & $\begin{array}{l}\text { velocity or energy distribution } \\
\text { function }\end{array}$ & \\
\hline$h$ & Planck's constant & $6.626 \cdot 10^{-34} \mathrm{~J} \cdot \mathrm{s}$ \\
\hline$H_{\mathrm{f}}$ & reaction enthalpy & {$[\mathrm{J} / \mathrm{mol}]$} \\
\hline I & current & [A] \\
\hline I & $\begin{array}{l}\text { intensity of a particular emission } \\
\text { line }\end{array}$ & {$[\mathrm{W} / \mathrm{sr}]$} \\
\hline$I_{\mathrm{e} 0}, I_{\mathrm{i} 0}$ & electron or ion saturation current & [A] \\
\hline$J$ & current density & {$\left[\mathrm{A} / \mathrm{cm}^{2}\right]$} \\
\hline$k$ & reaction rate constant & {$\left[\mathrm{m}^{3} \cdot \mathrm{s}^{-1}\right]$} \\
\hline
\end{tabular}




\begin{tabular}{|c|c|c|}
\hline Symbol & Description & (value) [unit] \\
\hline$k$ & wave number & {$\left[\mathrm{m}^{-1}\right]$} \\
\hline$k_{\mathrm{iz}}$ & rate constant for ionization & {$\left[\mathrm{m}^{3} \cdot \mathrm{s}^{-1}\right]$} \\
\hline$k_{\mathrm{m}}$ & mean thermal conductivity & {$\left[\mathrm{J} \cdot \mathrm{m}^{-1} \cdot \mathrm{s}^{-1} \cdot \mathrm{K}^{-1}\right]$} \\
\hline$k_{\text {rec }}$ & rate constant for recombiation & {$\left[\mathrm{m}^{3} \cdot \mathrm{s}^{-1}\right]$} \\
\hline$K_{\mathrm{ox}}$ & oxide dielectric constant & \\
\hline$k T_{\mathrm{e}}$ & characteristic electron energy & {$[\mathrm{eV}]$} \\
\hline$m_{\mathrm{e}}$ & electron mass & $9.109 \cdot 10^{-31} \mathrm{~kg}$ \\
\hline$n$ & Index of refraction & \\
\hline$N_{\mathrm{e}, \mathrm{i}, \mathrm{g}}$ & electron, ion, or neutral density & {$\left[\mathrm{m}^{-3}\right]$} \\
\hline$Q$ & throughput & {$\left[\right.$ Torr $\cdot$ liter $\cdot \mathrm{s}^{-1}$ ] } \\
\hline$Q_{\text {bulk }}$ & oxide bulk charge & {$\left[\mathrm{C} / \mathrm{cm}^{2}\right]$} \\
\hline$Q_{\mathrm{f}}$ & $\begin{array}{l}\text { Fixed charge near the } \mathrm{Si}-\mathrm{SiO}_{2} \text { in- } \\
\text { terface }\end{array}$ & {$\left[\mathrm{cm}^{-2}\right]$} \\
\hline$Q_{\mathrm{s}}$ & substrate charge & {$\left[\mathrm{C} / \mathrm{cm}^{2}\right]$} \\
\hline$R_{\mathrm{amb}}$ & $\begin{array}{l}\text { loss rate of ions and electrons due } \\
\text { to ambipolar diffusion }\end{array}$ & {$\left[\mathrm{s}^{-1}\right]$} \\
\hline$R_{\mathrm{p}}$ & projected range & [nm] \\
\hline$R_{\text {rec }}$ & recombination rate & {$\left[\mathrm{s}^{-1}\right]$} \\
\hline$S$ & $\begin{array}{l}\text { production rate of ions and elec- } \\
\text { trons }\end{array}$ & {$\left[\mathrm{s}^{-1}\right]$} \\
\hline$S$ & pumping speed & {$[1 / \mathrm{s}]$} \\
\hline$T_{\mathrm{ox}}$ & thickness of the oxide & [nm] \\
\hline$V_{\mathrm{f}}$ & floating potential & [V] \\
\hline$V_{\mathrm{FB}}$ & flatband voltage & [V] \\
\hline$V_{\mathrm{G}}$ & gate voltage & [V] \\
\hline$V_{\text {ox }}$ & potential drop across the oxide & [V] \\
\hline$V_{\mathrm{p}}$ & plasma potential & [V] \\
\hline$\alpha$ & $\begin{array}{l}\text { thermal accommodation coeffi- } \\
\text { cient }\end{array}$ & \\
\hline$\alpha_{\mathrm{iz}}$ & ionization degree & \\
\hline$\Gamma$ & particle flux & {$\left[\mathrm{m}^{-2} \cdot \mathrm{s}^{-1}\right]$} \\
\hline $\begin{array}{l}\Delta R_{p} \\
\varepsilon\end{array}$ & $\begin{array}{l}\text { projected straggle } \\
\text { emission coefficient due to radia- } \\
\text { tion }\end{array}$ & [nm] \\
\hline$\varepsilon_{0}$ & permittivity of vacuum & $8.854 \cdot 10^{-12} \mathrm{~F} / \mathrm{m}$ \\
\hline$\theta$ & implantation dose & [ions $\left./ \mathrm{cm}^{2}\right]$ \\
\hline$\lambda_{\mathrm{D}}$ & $\begin{array}{l}\text { Debye length or shielding dis- } \\
\text { tance }\end{array}$ & [m] \\
\hline
\end{tabular}




\begin{tabular}{lll}
\hline Symbol & Description & (value) $[\mathrm{unit}]$ \\
\hline$\lambda_{\mathrm{m}}$ & $\begin{array}{l}\text { mean free path between collisions } \\
\text { mobility constant of the electrons }\end{array}$ & {$[\mathrm{m}]$} \\
$\mu_{\mathrm{e}, \mathrm{i}}$ & $\left.\left[\mathrm{m}^{2} / \mathrm{\textrm {V }} \cdot \mathrm{s}\right)\right]$ \\
& $\begin{array}{l}\text { (e) or ions (i) } \\
v\end{array}$ & {$[\mathrm{~Hz}]$} \\
$\rho$ & collision frequency & {$\left[\mathrm{C} / \mathrm{cm}^{3}\right]$} \\
$\rho_{\text {ion }}$ & charge density & {$\left[\mathrm{ions} / \mathrm{cm}^{3}\right]$} \\
$\rho_{\mathrm{ox}}$ & ion density in the oxide & {$\left[\mathrm{C} / \mathrm{cm}^{3}\right]$} \\
$\sigma$ & charge density in the oxide & {$\left[\mathrm{m}^{2}\right]$} \\
& cross section of a particular colli- \\
$\sigma_{\mathrm{B}}$ & sion process & \\
$\tau$ & Stefan-Boltzmann constant & $5.67 \cdot 10^{-8} \mathrm{~W} \cdot \mathrm{m}^{-1} \cdot \mathrm{K}^{-4}$ \\
$\tau$ & mean time between collisions & {$[\mathrm{s}]$} \\
$v_{\mathrm{B}}$ & residence time & {$[\mathrm{s}]$} \\
$v_{\mathrm{e}}$ & Bohm velocity & {$\left[\mathrm{m} \cdot \mathrm{s}^{-1}\right]$} \\
$\phi$ & electron velocity & {$\left[\mathrm{m} \cdot \mathrm{s}^{-1}\right]$} \\
$\Phi$ & photon flux & {$[\mathrm{photons} / \mathrm{s}]$} \\
$\varphi_{\mathrm{ms}}$ & potential & {$[\mathrm{V}]$} \\
$\Psi_{\mathrm{s}}$ & metal-semiconductor workfunc- & {$[\mathrm{V}]$} \\
$\omega$ & tion difference & \\
$\omega_{\mathrm{pe}} / \omega_{\mathrm{pi}}$ & semiconductor surface potential & {$[\mathrm{V}]$} \\
\hline & oscillation frequency & {$[\mathrm{Hz}]$} \\
& electron/ion plasma frequency & {$[\mathrm{Hz}]$} \\
\hline
\end{tabular}




\begin{tabular}{ll}
\hline Acronym & Description \\
\hline AES & Auger electron spectroscopy \\
ALD & atomic layer deposition \\
CCD & charge-coupled device \\
CCP & capacitively coupled plasma \\
CL & cathodoluminescence \\
CMOS & complementary metal-oxide-semiconductor \\
CVD & chemical vapor deposition \\
dc & direct current \\
ECR & electron cyclotron resonance \\
EEDF & electron energy distribution function \\
ETP & expanding thermal plasma \\
FN & Fowler-Nordheim (tunneling current) \\
HDRP & high-density remote plasma \\
HF & high-frequency or Hydrofluoric acid \\
ICP & inductively coupled plasma \\
ICPECVD & inductively-coupled plasma-enhanced chemical vapor depo- \\
& sition \\
LP & Langmuir probe \\
LPCVD & low-pressure chemical vapor deposition \\
LSS & Lindhard, Scharff, Schiott \\
MB & Maxwell-Boltzmann (not: Mercedes Benz) \\
MEMS & Micro-electro-mechanical systems \\
MOEMS & Micro-opto-electromechanical systems \\
MOS & metal oxide semiconductor \\
NIR & near-infrared \\
NVM & non-volatile memory \\
OES & Optical emission spectroscopy \\
PECVD & plasma-enhanced chemical vapor deposition \\
PID & proportional-integral-derivative \\
PL & photoluminescence \\
PMA & post-metal anneal \\
PO & plasma oxidation \\
POA & post-oxidation anneal \\
QS & quasi-static \\
RESURF & reduced surface field \\
rf & radio frequency \\
RP & remote plasma \\
\hline &
\end{tabular}




\begin{tabular}{ll}
\hline Acronym & Description \\
\hline RTO & rapid thermal oxidation \\
SE & spectroscopic ellipsometry \\
SEM & scanning electron microscopy \\
SHG & second-harmonic generation \\
TEM & transmission electron microscopy \\
TEOS & Tetraethyl orthosilicate \\
TFT & thin-film transistor \\
TRG-OES & trace rare gasses optical emission spectroscopy \\
XPS & x-ray photoelectron spectroscopy \\
XRD & x-ray diffraction \\
XRR & x-ray reflectometry \\
\hline
\end{tabular}




\section{Introduction}

\subsection{Motivation and scientific challenges}

The development of electronic integrated circuits has had and still has a large impact on society, enabling a highly automated world. Most of the electronic integrated circuits used today are made in complementary metal-oxide-semiconductor (CMOS) technology. The whole of CMOS industry has been made possible by the unique properties of silicon dioxide: the only native oxide of a common semiconductor which is stable in water and at elevated temperatures, an excellent electrical insulator, a good dielectric, a mask to common diffusing species and capable of forming a nearly perfect electrical interface with its substrate when it is grown by thermal oxidation (at $800-1100^{\circ} \mathrm{C}$ ) [1]. Deposited silicon dioxide, manufactured by chemical vapor deposition (CVD), is almost as old as thermal growth on the substrate, and has been employed in various ways in IC fabrication due to its familiarity, versatility and reliability [2]. Lowpressure chemical vapor deposition (LPCVD), and plasma-enhanced chemical vapor deposition (PECVD) are the major CVD methods nowadays. While LPCVD is operated between $600-800^{\circ} \mathrm{C}$, PECVD is typically applied at $400^{\circ} \mathrm{C}$ and lower temperatures [2].

Metal interconnects are introduced at some stage in the CMOS fabrication process (the so-called "back-end of line"). Thermal stability demands that the inter-metal dielectrics (often silicon dioxides) are deposited with a maximum temperature of about $400^{\circ} \mathrm{C}$ [3], and PECVD allows just that. Rather than relying solely on thermal energy to initiate and sustain chemical reactions, PECVD uses an rf-induced glow discharge to transfer energy into the 
reactant gases, allowing the substrate to remain at a lower temperature (i.e., $150-400^{\circ} \mathrm{C}$ ) than in LPCVD. Lower substrate temperature is the major advantage of PECVD, and in fact, PECVD provides a method of depositing films on substrates that do not have the thermal stability to accept coating by other methods.

Other depositing techniques are developed to form silicon-based films at low substrate temperatures: photon-induced CVD, catalytic CVD, (reactive) sputtering, jet vapor deposition, pulsed-laser deposition, (plasma assisted) atomic layer deposition, sol-gel techniques, and the like [4]. PECVD processes are often selected over competing techniques because they offer (one of) the following advantages: (a) high purity films can be achieved; (b) great variety of compositions can be deposited; (c) some films cannot be deposited with adequate film properties by any other method; and (d) good economy and process control are possible for many films. That is why PECVD is nowadays one of the key sectors of the industrial production of silicon-based films, notably, CMOS, thin film transistors, display technology and solar cells [2].

However, the temperature reduction by PECVD has its price in a deteriorated layer quality. Reactions are not completed (i.e. not all the reaction products can desorb from the film), and highly energetic plasma particles such as ions, electrons and photons bombard the surface of the wafer continuously. These bombardments can improve the properties of the film, but they can cause many types of damage to the growing film and to the underlying layers when they are too severe.

The term "layer quality" used above refers here to some of the electrical and physical characteristics of the silicon dioxide layers: leakage current, dielectric strength, fixed and mobile oxide charge, defect density at the interface with silicon, etch rate and index of refraction. The quality of thermally grown silicon dioxide layers act as the benchmark. Important properties of CVD and thermal silicon dioxide are summarized in Table 1.1.

The industrial sector stimulates research and development of the deposition processes and their physical chemistry. Enhancement of the layer quality, reduction of plasma induced damage and lower deposition temperatures are important issues. R\&D efforts bear on many disciplines: reactor design, fluid mechanics, plasma physics and chemistry, gas-surface interactions to mention but a few. In this context, it must be noted that the engineering and optimization of reactors and deposition processes is much too costly and timeconsuming to be left to the sole approach of trial and error, whereby various recipes are tested and then flow rates, temperatures and discharge currents adjusted while growth rates and film quality are being monitored. The impor- 


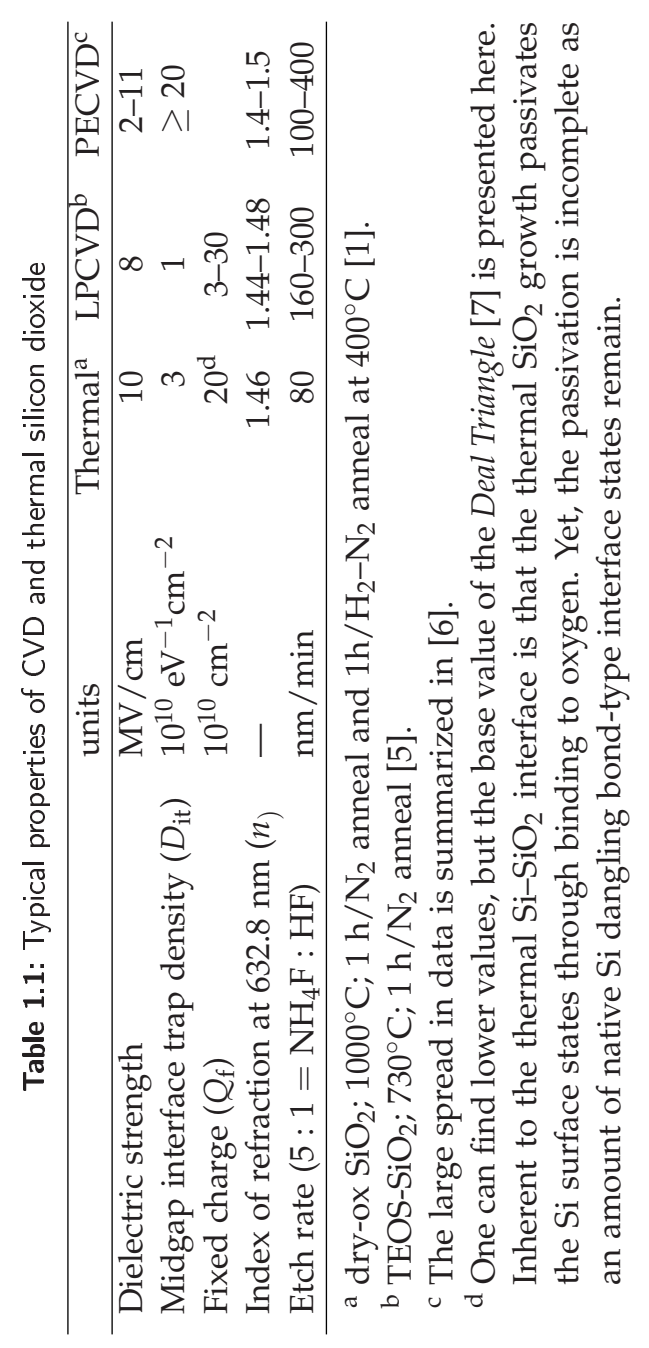


tance of fully integrated PECVD simulators (Virtual Reactors) is recognized by various research groups and scientific software companies [8].

Modeling and simulation of the PECVD process is essentially a multidisciplinary endeavor and the state-of-the-art is far from satisfactory. Models used to obtain important plasma parameters are detailed and sophisticated but do still not accurately describe e.g. plasma non-uniformities and electron energy distribution functions (EEDFs) for every type of reactor and all process conditions [9-15]. Yet academic research laboratories would greatly benefit from these models. Indeed, it is precisely in these places that some of the key expertise is found. They have the competence needed to study the complex chemical reactions; they understand discharge mechanisms and can propose and test new precursors. The applied mathematics laboratories are now in a position to cooperate with them fruitfully and to allow them to accomplish great progress, by offering advanced open tools of simulation. The work presented in this thesis was launched along this line.

The goal we set out to reach was to contribute to PECVD technology by showing its capability to fabricate high-quality silicon dioxide gate dielectrics. Our second objective was to contribute to a more exact understanding of PECVD technology by showing that an accurate description of plasma parameters and chemistry can assist researchers to find optimal process conditions. We defined a specific PECVD process - the deposition of silicon dioxide films from Ar$\mathrm{N}_{2} \mathrm{O}-\mathrm{SiH}_{4}$ plasmas - to mention a few points that are of general interest. First, we demonstrate that we can deposit high-quality silicon dioxide dielectrics. These sound layers were characterized quite extensively since they show features which are of direct importance for industry and academic communities. We also demonstrate that we forecast at which process conditions these sound films are formed. This forecast is based on modeling of chemical reactions in silane-containing plasmas, which again relies on the measurement of correct plasma parameters. We show that the use of an incorrect EEDF, which, at first glance, deviates only slightly from the actual distribution, can cause strong disagreements between the actual processes and the model. Virtual Reactor PECVD simulators can benefit from this knowledge by allowing experimental data of the plasma state into their code, while it might encourage physicists to work on more precise plasma models.

\subsection{Background}

This subchapter provides a short overview of gas discharges and PECVD processes to guide the reader through the topics treated in this thesis (plasma 


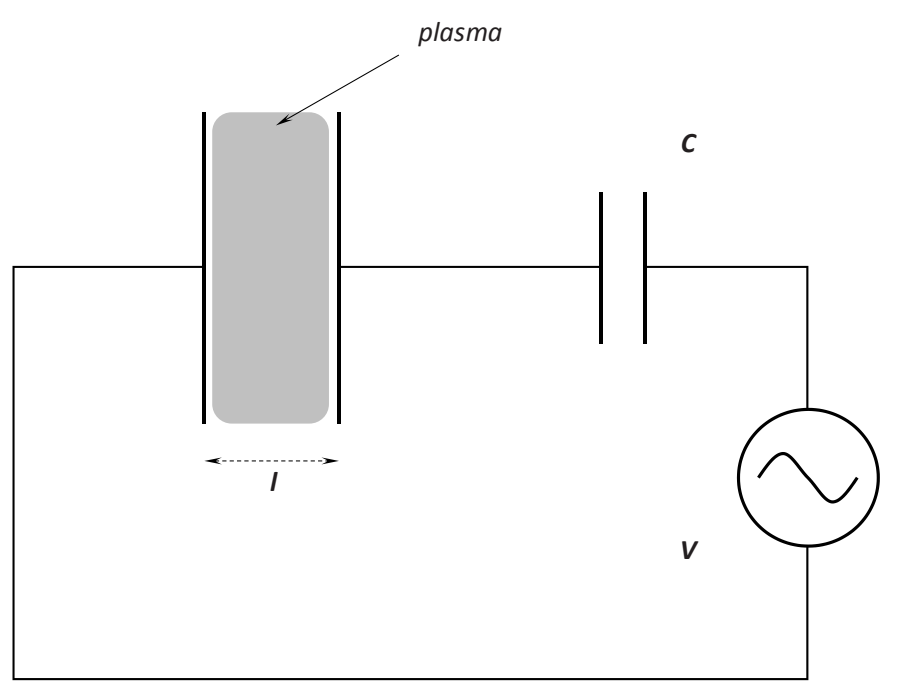

Figure 1.1: Schematic drawing of a capacitively coupled rf plasma.

characterization, the related modeling as well as the characterization and application of the deposited layers).

\subsubsection{Developments in plasma sources}

Plasma is ionized gas consisting of a mixture of free electrons, ions and neutrals (depending on the degree of ionization) and it is formed when sufficient energy is dissipated in a gas [16]. More than $90 \%$ of matter in the universe exists in the plasma state $[17,18]$ though nature rarely produces plasma on the earth's surface. In nature plasma is formed when gas is exposed to high temperatures (inside of stars) or high energy radiation (UV radiation and cosmic ray particles in the ionosphere). Under laboratory conditions however, where such high energies are not easily available, ionized gas is generated using high electric field. This is known as cold plasma or electric discharge. Electrical discharge is the most practical means of creating and sustaining low temperature plasma in the laboratory and many methods of coupling electrical energy into gases to generate a plasma have been developed using both direct current (dc) as well as alternating current (ac) power sources.

The most commonly used plasma reactor of today is the capacitively coupled radio-frequency (rf) discharge. The reactor consists of two parallel 
electrodes, separated at a distance $l$, see Figure 1.1. The substrate is placed on one of these electrodes. The most common rf frequency is $13.56 \mathrm{MHz}$, an allowed frequency which does not interfere with radio and television broadcasting bands. The gap between the electrodes is filled with a gas. At 13.56 $\mathrm{MHz}$, the ions are not able to follow the electromagnetic field, whereas the electrons are. Therefore, the electromagnetic energy will be mainly absorbed by the electrons, which can ionize the neutrals. The ions are much slower than the electrons due to their mass. This results in a faster loss of negative charge to the electrodes. The resulting potential difference between the electrodes and the plasma creates an electric field region at the edge of the plasma. This region, the sheath, accelerates the ions and slows down the electrons, balancing the fluxes of positively and negatively charges particles to the electrodes. As a result, the ions will reach the electrodes, and therefore the substrate, with a high energy. Most of the surface chemistry occurs due to the high energy ion bombardment. A higher electron density results in a higher rate of electron-stimulated reactions in the gas phase. To produce more dense plasma, the electrode potential has to increase. Simultaneously the ion energy at the electrode surface increases, which can cause unwanted effects, such as film damage. The higher plasma density also increases radical interactions which gives rise to the formation of particles in the gas phase (gas-phase nucleation). These limitations cause a relatively narrow process window for many applications and have led to the development of remote plasma (RP) processes [19].

Here, the substrate is placed outside (remote from) the plasma generation region and is separately biased from the electronic circuit that generates the plasma. Thus, separate rf sources are used to control both the plasma density and the ion bombarding energy. On top of that, plasma sources are developed which can operate at lower pressures but can induce higher plasma densities, i.e., high density remote plasma (HDRP) reactors [19]. The operational conditions and the resulting plasma parameters are shown in Table 1.2. They are compared with the values for the capacitively coupled plasma (CCP) sources.

Figure 1.2 shows some examples of high density remote plasma reactors. First, the electron cyclotron resonance (ECR) plasma reactor is shown [21]. Here, one or more permanent magnets surround the plasma chamber to create a dc, axially varying magnetic field. Microwave power is injected into the plasma through a dielectric window. This power is absorbed by the electrons, which can ionize the gas in the chamber. The helicon source uses a dc magnetic field in combination with an rf driven antenna [22]. The excited helicon wave interacts with the electrons in the chamber, resulting in a transfer of energy. The Expanding Thermal Plasma (ETP) technique [20] operates in a quite different way. 
Table 1.2: Typical values for CCP and HDRP sources [19-22].

\begin{tabular}{llcc}
\hline parameter & units & CCP source & HDRP source \\
\hline Pressure $p$ & $\mathrm{~Pa}$ & $1-100$ & $0.05-20$ \\
Applied power $P$ & $\mathrm{~W}$ & $50-2000$ & $100-5000$ \\
Frequency $f$ & $\mathrm{MHz}$ & $0.05-13.56$ & $0-2450$ \\
Plasma density $N$ & $\mathrm{~m}^{-3}$ & $10^{15}-10^{17}$ & $10^{15}-10^{18}$ \\
Electron temperature $k T_{\mathrm{e}}$ & $\mathrm{eV}$ & $1-5$ & $0.3-7$ \\
Ion energy $\varepsilon_{\mathrm{i}}$ & $\mathrm{eV}$ & $200-1000$ & $20-500$ \\
Ionization degree $\alpha_{\mathrm{iz}}$ & - & $10^{-6}-10^{-3}$ & $10^{-4}-10^{-1}$ \\
\hline
\end{tabular}

The ETP setup consists of a high-pressure plasma source and a low-pressure chamber. The dc plasma source is a cascaded arc. In this plasma source reactive species are created that can be used for downstream precursor gas dissociation for plasma deposition. The plasma in the arc is thermal with an electron density of $\sim 10^{22} \mathrm{~m}^{-3}$ and with an electron and heavy particle temperature of both $\sim 1 \mathrm{eV}$. The plasma emanates from the cascaded arc source through a nozzle and expands into the deposition chamber which is typically at a pressure of $20 \mathrm{~Pa}$. Due to the large difference in pressure between the arc and the chamber, the plasma expands subsonically. Eventually, the electron temperature is reduced to $0.3 \mathrm{eV}$ and the electron density to $\sim 10^{17}-10^{19} \mathrm{~m}^{-3}$. The last source in Figure 1.2 is the inductively coupled plasma (ICP) source [21]. The cylindrical coil and the plasma together act as a transformer, where the plasma is the secondary, single turn, conductor. The power is inductively coupled through a dielectric window without using external magnets. This source is used in this thesis. Inductively coupled discharges are now the leading plasma sources for plasma processing because of the simplicity of concept, easy scalability, and no requirement of constant magnetic field, which is necessary in ECR and helicon discharges $[19,22]$.

\subsubsection{PECVD}

A typical PECVD reactor is a low-pressure plasma chamber of a few liters in volume, through which silane gas $\left(\mathrm{SiH}_{4}\right)$, often diluted with other precursors and argon, is pumped at a certain flow rate. A gas mixture enters the reactor at room temperature. A substrate for film deposition is located in the chamber. The substrate can be part of the electrical circuit that drives the discharge (direct PECVD) or is electrically separated from the electronic circuit that generates the plasma (remote PECVD). An rf oscillating voltage is applied to an electrode inside or outside the chamber. A number of intertwined processes occur in a 

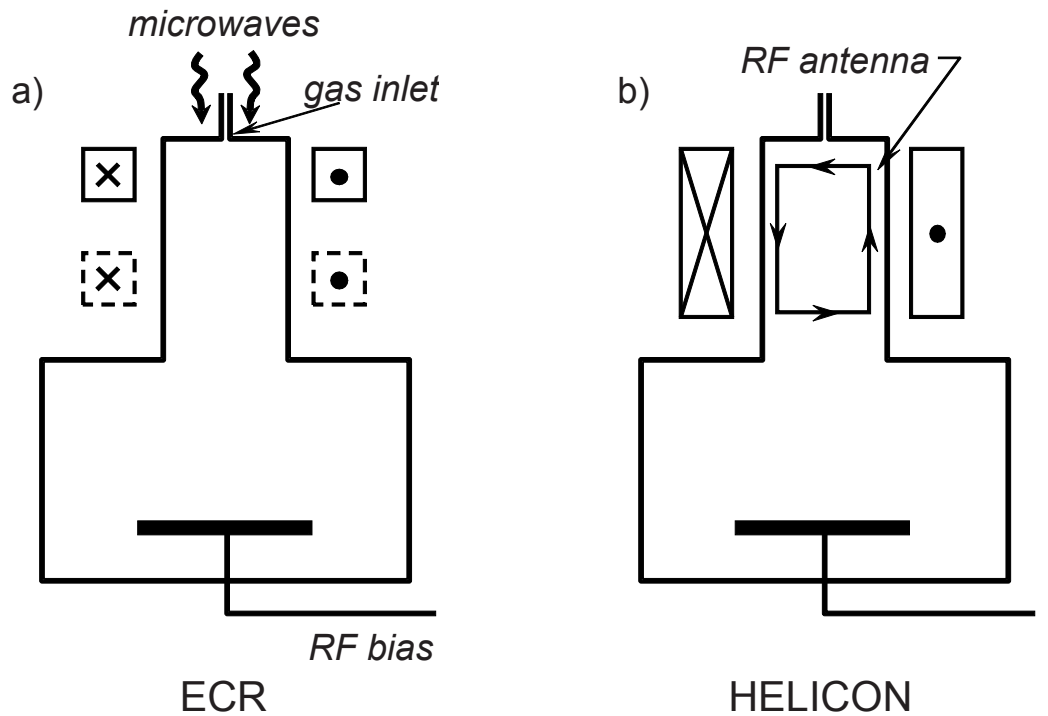

HELICON

c)

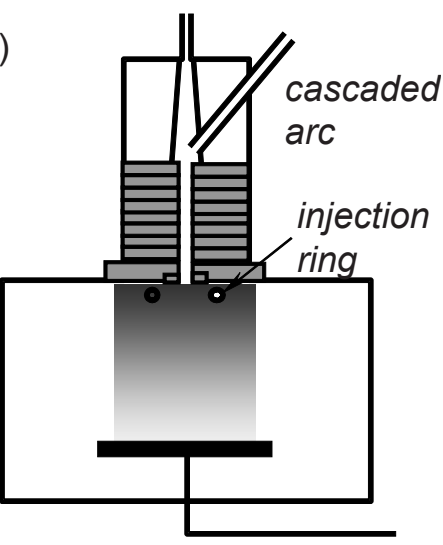

ETR

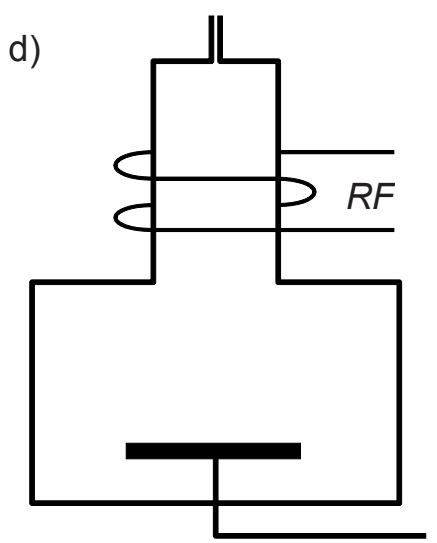

Inductive

Figure 1.2: Four examples of HDRP sources. 
PECVD reactor, which can be simplified by the following scheme:

1. A glow discharge initiates the ionization and dissociation processes by electron impact.

2. Gas phase reaction and transport of the reactive species by diffusion to the wafer surface.

3. Adsorption of reactive species on the wafer surface sites and the reaction of these species with surface atoms or with other adsorbed species to form a reaction product.

4. Surface diffusion of the adsorbed species and reaction products. Nuclei grow into islands and islands merge into a continuous thin film.

5. Desorption of the volatile reaction products and transport of byproducts away from the growth region.

Ion bombardment aids processes 3,4 and 5. The quality of the growing films depends, among others, on the percentage of different species contributing to the growth. A deeper insight into this aspect of the deposition processes is another aspect of this thesis.

\subsection{Objectives of this research}

The aim is to deposit silicon dioxide layers with excellent quality at substrate temperatures of $150^{\circ} \mathrm{C}$ in a remote ICPECVD reactor. A combination of theory and empirical approaches was used. Motivated by the important role of plasmas in technology and the need for simulations to understand the associated complex processes, it was attempted to combine in one research plasma characterization, chemical modeling and the (electrical) properties of the deposited films. In this way, we hope to gain insight into the deposition processes, to contribute to the optimization of PECVD reactors, and to enable the fabrication of electronic devices at low substrate temperatures.

\subsection{Outline of the thesis}

This thesis is divided in two parts. Part I is devoted to the plasma state. The ICPECVD system is introduced and the plasma it produces is characterized with respect to the electron density and electron energy distribution function. These parameters were used to model the composition of $\mathrm{Ar}-\mathrm{N}_{2} \mathrm{O}-\mathrm{SiH}_{4}$ plasmas. Attempts were made to predict the electrical and physical properties of deposited $\mathrm{SiO}_{2}$ films from the modeled plasma composition. The accuracy of the modeling was tested for a particular process window. This window was 
chosen such that minimal variations in total pressure (1-6 Pa) and initial silane concentration $(0.08-0.8 \%)$ resulted in predicted maximum variations in plasma composition. Part II of this thesis is devoted to the deposition and characterization of these films. Correlations between predicted and measured properties are drawn. Besides that, the layers with the best dielectric properties are employed in thin film transistors to confirm the quality of the films. 
PART I

THE PLASMA STATE 


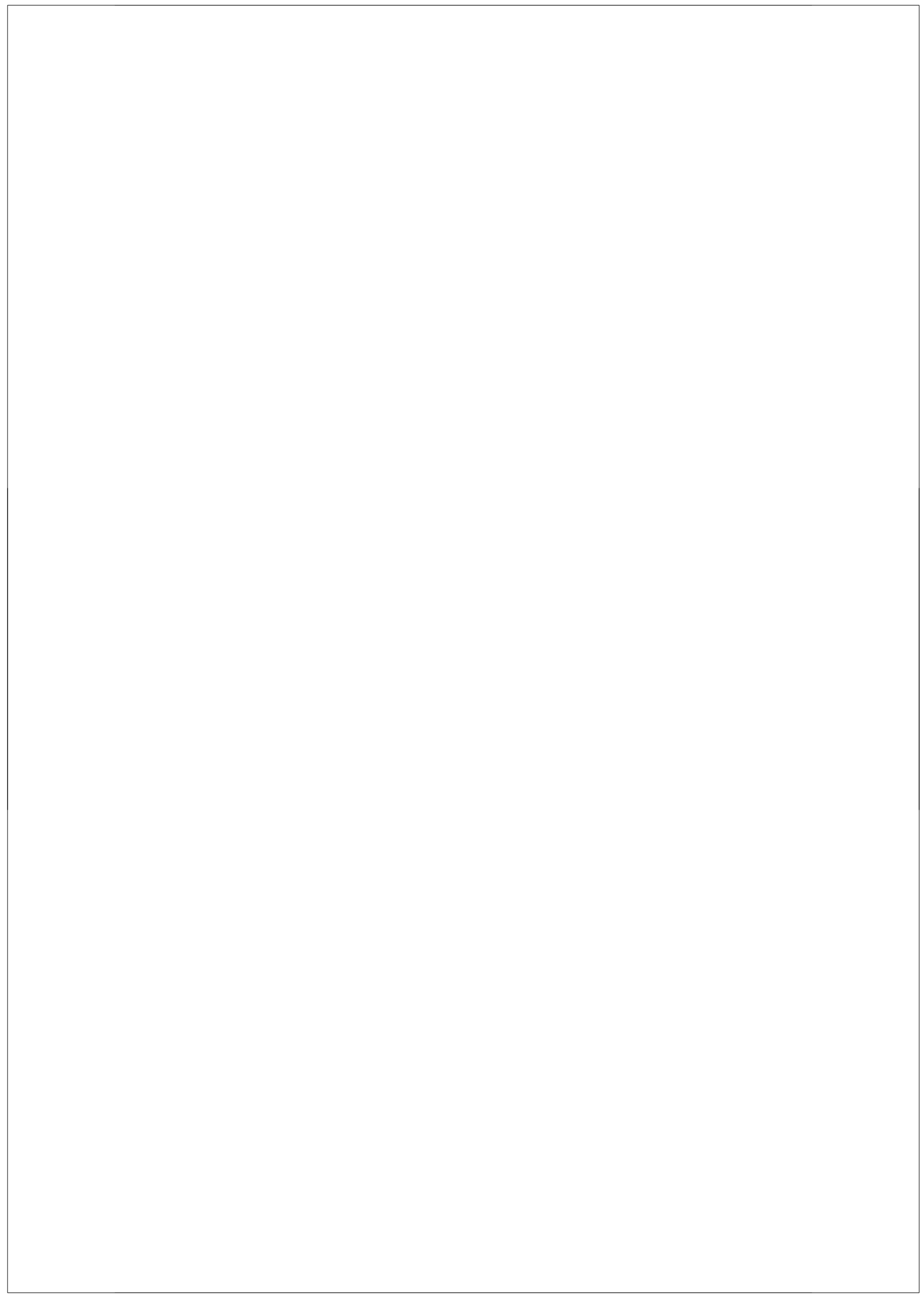




\section{Introduction}

An overview is given of the relevant plasma physics and the various aspects of plasma analysis via Langmuir probe and optical emission measurements. The plasma reactor is introduced and characterized with respect to electron energy distribution functions (EEDF) and electron densities. These two parameters are of prime importance for the modeling and optimization of chemical and physical processes in plasma reactors. An approach is developed to obtain EEDFs for plasmas with silane precursor for thin film deposition of silicon dioxide from $\mathrm{Ar}-\mathrm{N}_{2} \mathrm{O}-\mathrm{SiH}_{4}$ mixtures. This approach allows for modeling of depositing plasmas where experimental data are used as input parameters, thus minimizing modeling errors. The latter is essential for a better understanding and optimization of the deposition process. Promising results of this approach are demonstrated. 


\section{Aspects of plasma physics}

An overview is given of the relevant plasma physics. Various plasma parameters are calculated and evaluated for an argon discharge at process conditions typical for HDRP sources (cf. Table 1.2).

\subsection{General}

A plasma is a quasi-neutral gas of neutrals, ions, and electrons which exhibits collective behavior $[16,23]$. To classify the various plasma configurations, the ionization degree $\alpha_{\mathrm{iz}}$ is often used:

$$
\alpha_{\mathrm{iz}}=\frac{N_{\mathrm{i}}}{N_{\mathrm{i}}+N_{\mathrm{g}}}
$$

where $N_{\mathrm{i}}$ and $N_{\mathrm{g}}$ are the ion and neutral gas densities in the plasma. This quantity is unity for a fully ionized plasma (e.g. thermo nuclear plasmas [24]) and much smaller (in the order of $10^{-2}$ to $10^{-6}$ ) for weakly ionized plasmas. The low-pressure discharges created in standard reactors are all weakly ionized plasmas. The neutrals, ions and electrons all have their own energy distribution. Direct Monte Carlo simulation of electron kinetics showed that at pressures higher than 0.1 Torr (13 Pa) we can use a gas-dynamic approach for electron and ion description [14,25]. Typical pressures in this research are somewhat lower (1-6 Pa) but for now we will use the same approach and discuss the validity later.

Because the energy exchange due to elastic collisions between neutrals and electrons and between ions and electrons is very inefficient due to the 
large mass ratio, the electrons have a much higher average energy than the ions and neutrals. At sufficiently high electron-electron collision frequencies, the velocities $v_{\mathrm{e}}$ of the electrons (with mass $m_{\mathrm{e}}$ ) are distributed according to the Maxwell-Boltzmann distribution function $f_{v}$ with a characteristic temperature $T_{\mathrm{e}}[26]$ :

$$
f_{v}\left(v_{\mathrm{e}}\right) \cdot \mathrm{d} v_{\mathrm{e}}=\frac{4}{\sqrt{\pi}}\left(\frac{m_{\mathrm{e}}}{2 k T_{\mathrm{e}}}\right)^{3 / 2} v_{\mathrm{e}}^{2} \exp \left(-\frac{m_{\mathrm{e}} v_{\mathrm{e}}^{2}}{2 k T_{\mathrm{e}}}\right) \cdot \mathrm{d} v_{\mathrm{e}} .
$$

Here $k$ is the Boltzmann constant and $e$ is the electron charge. According to the relation above, $N_{\mathrm{e}} f_{v}\left(v_{\mathrm{e}}\right) \mathrm{d} v_{\mathrm{e}}$ represents the number of electrons having a velocity in the interval $\left[v_{\mathrm{e}}, v_{\mathrm{e}}+\mathrm{d} v_{\mathrm{e}}\right]$. The average electron velocity is found by integration:

$$
\left\langle v_{\mathrm{e}}\right\rangle=\int_{0}^{\infty} v_{\mathrm{e}} f_{v}\left(v_{\mathrm{e}}\right) \mathrm{d} v_{\mathrm{e}}=\sqrt{\frac{8 k T_{\mathrm{e}}}{\pi m_{\mathrm{e}}}},
$$

and can be evaluated to measure $9.5 \cdot 10^{5} \mathrm{~m} / \mathrm{s}$ for a distribution with a characteristic temperature of $2 \mathrm{eV}$. The maximum of $f_{v}\left(v_{\mathrm{e}}\right)$ is given by $\frac{1}{2} m_{\mathrm{e}} v_{\mathrm{e}}^{2}=k T_{\mathrm{e}}$. The distribution function can also be expressed in terms of energy:

$$
f_{\mathrm{E}}\left(E_{\mathrm{e}}\right) \cdot \mathrm{d} E_{\mathrm{e}}=\frac{2}{\sqrt{\pi}}\left(\frac{1}{k T_{\mathrm{e}}}\right)\left(\frac{E_{\mathrm{e}}}{k T_{\mathrm{e}}}\right)^{1 / 2} \exp \left(-\frac{E_{\mathrm{e}}}{k T_{\mathrm{e}}}\right) \cdot \mathrm{d} E_{\mathrm{e}} .
$$

The maximum of the energy distribution is found at $E_{e}=\frac{1}{2} k T_{\mathrm{e}}$. The average energy is $\frac{3}{2} k T_{\mathrm{e}}$. Typical electron temperatures in low-pressure plasmas vary from 104 to $105 \mathrm{~K}$. This corresponds to kinetic energies of $1-10 \mathrm{eV}$. These are much higher than the temperature of the neutrals (e.g. $300 \mathrm{~K} \approx 0.026 \mathrm{eV}$ ) or ions (e.g. $500 \mathrm{~K} \approx 0.04 \mathrm{eV}$ ).

The random flux $\Gamma$ of particles in an arbitrary direction [27] is given by

$$
\Gamma_{\mathrm{e}, \mathrm{i}}=\frac{1}{4} N_{\mathrm{e}, \mathrm{i}}\left\langle v_{\mathrm{e}, \mathrm{i}}\right\rangle,
$$

with $N_{\mathrm{e}, \mathrm{i}}$ the density and $\left\langle v_{\mathrm{e}, \mathrm{i}}\right\rangle$ the average velocity of the electrons (e) and ions (i). Since the average velocity of electrons is much larger than that of the ions, an electrically insulated object in the plasma will initially be bombarded with more electrons than ions. Therefore, the object will be charged negatively, until the resulting electric field is strong enough to balance the electron and ion fluxes. The region where electrons are repelled and ions are attracted is called "the sheath". A sheath is present around every insulated object in contact with the 
plasma, like walls and probes. The equilibrium potential of the object is called the floating potential, $V_{\mathrm{f}}$. The definition of this potential is only meaningful if it is compared with the plasma potential, $V_{\mathrm{p}}$. When the object is at floating potential, the ion and electron flux balance. The electron flux is smaller than the electron thermal flux, $N_{\mathrm{e}} \frac{1}{4}\left\langle v_{\mathrm{e}}\right\rangle$, due to the retarding field, while the ion flux is determined by the thermal flux at the edge of the sheath [28]:

$$
\frac{1}{4} N_{\mathrm{e}}\left\langle v_{\mathrm{e}}\right\rangle \exp \left(-\frac{e\left(V_{\mathrm{p}}-V_{\mathrm{f}}\right)}{k T_{\mathrm{e}}}\right)=N_{\mathrm{i}} \frac{1}{4}\left\langle v_{\mathrm{i}}\right\rangle,
$$

resulting in

$$
V_{\mathrm{p}}-V_{\mathrm{f}}=\frac{k T_{\mathrm{e}}}{2 e} \ln \left(\frac{m_{\mathrm{i}}}{m_{\mathrm{e}}} \frac{T_{\mathrm{e}}}{T_{\mathrm{i}}}\right) .
$$

This relation can be refined when the Bohm sheath criterion is taken into account [29]. According to this criterion, ions have to be pre-accelerated up to an energy $\frac{1}{2} k T_{\mathrm{e}}$ before they reach the plasma-sheath boundary, in order to maintain a positive space charge everywhere in the sheath [29]. This implies the existence of a presheath region with negligible space charge $\left(N_{\mathrm{i}} \approx N_{\mathrm{e}}\right)$ but with a potential drop of $V=k T_{\mathrm{e}} / 2 e$ relative to the plasma. When $N_{\mathrm{e}}$ represents the electron density in the bulk, the density $N_{\mathrm{e}}(d)$ at the plasma sheath boundary is then given by the Boltzmann relation:

$$
N_{\mathrm{e}}(d)=N_{\mathrm{e}} \mathrm{e}^{-V / k T_{\mathrm{e}}}=N_{\mathrm{e}} \mathrm{e}^{-0.5} \approx 0.6 N_{\mathrm{e}}
$$

The ions accelerate in the presheath to the Bohm velocity,

$$
v_{\mathrm{B}}=\sqrt{\frac{k T_{\mathrm{e}}}{m_{\mathrm{i}}}},
$$

and because $N_{\mathrm{i}}(d) \approx N_{\mathrm{e}}(d)$, the ion flux is given by

$$
\Gamma_{\mathrm{i}} \approx 0.6 N_{\mathrm{i}} v_{\mathrm{B}}
$$

When the Bohm velocity is used on the right hand side of equation (3.6) instead of the thermal ion velocity, equation (3.7) alters in

$$
V_{\mathrm{p}}-V_{\mathrm{f}}=\frac{k T_{\mathrm{e}}}{2 e} \ln \left(\frac{m_{\mathrm{i}}}{2 \pi m_{\mathrm{e}}}\right)
$$

For argon, this results in $V_{\mathrm{p}}-V_{\mathrm{f}}=4.7 k T_{\mathrm{e}} / e$. 
To investigate the influence of a small charged object (e.g. an ion) on a homogeneous plasma, the one-dimensional Poisson equation is used to calculate the space dependence of the potential $\Phi(z)$ [29]:

$$
\frac{\mathrm{d}^{2} \Phi}{\mathrm{d} z^{2}}=-\frac{e\left(N_{\mathrm{i}}-N_{\mathrm{e}}\right)}{\varepsilon_{0}},
$$

where $\varepsilon_{0}$ is the permittivity of vacuum.

According to Boltzmann, the electron density is given by

$$
N_{\mathrm{e}}(z)=N_{\mathrm{e}}^{0} \exp \left(-\frac{e \Phi(z)}{k T_{\mathrm{e}}}\right)
$$

and the ion density is supposed to be constant:

$$
N_{\mathrm{i}}(z)=N_{\mathrm{i}}^{0},
$$

where $N_{\mathrm{i} 0}=N_{\mathrm{e} 0}=N_{0}$ are the original ion and electron densities in the plasma. Substituting this in the Poisson equation and assuming only small potential variations $\left(e \Phi(z) \ll k T_{\mathrm{e}}\right)$ :

$$
\frac{\mathrm{d}^{2} \Phi(z)}{\mathrm{d} z^{2}}=\frac{e N_{0}}{\varepsilon_{0}} \frac{e \Phi(z)}{k T_{\mathrm{e}}}
$$

This has an exponentially decaying solution:

$$
\Phi(z)=\Phi_{0} \exp \left(-\frac{z}{\lambda}\right)
$$

where $\Phi_{0}$ is the initial potential of the undisturbed plasma and

$$
\lambda=\sqrt{\frac{\varepsilon_{0} k T_{\mathrm{e}}}{e^{2} N_{0}}} \equiv \lambda_{\mathrm{D}},
$$

with $\pi_{\mathrm{D}}$ as the Debye length. This length parameter is the typical distance over which small potential variations stretch out over the plasma. The Debye length is $0.1 \mathrm{~mm}$ in a typical plasma with $k T_{\mathrm{e}}=2 \mathrm{eV}$ and an electron density of $10^{16}$ $\mathrm{m}^{-3}$. The Coulomb interaction between two particles (normally proportional to $r^{-1}$ ), is actually screened by the other charged particles [30]. This is called "Debye shielding", and $\lambda_{\mathrm{D}}$ "shielding distance". For length scales $l \gg \lambda_{\mathrm{D}}$, the plasma is quasi-neutral. 
When a disturbance in the plasma causes a small displacement $\zeta$ of the electrons with respect to the ions, the net space charge density on the edges is $e N_{\mathrm{e}} \zeta$ [29]. The resulting electric field is given by

$$
E=\frac{e N_{\mathrm{e}} \zeta}{\varepsilon_{0}} .
$$

The response of the electrons is

$$
m_{\mathrm{e}} \frac{\mathrm{d}^{2} \zeta}{\mathrm{d} t^{2}}=-e E
$$

or

$$
\frac{\mathrm{d}^{2} \zeta}{\mathrm{d} t^{2}}=-\omega_{\mathrm{pe}}^{2} \zeta
$$

This corresponds to an oscillatory motion with angular frequency $\omega_{\text {pe }}$ given by

$$
\omega_{\mathrm{pe}}=\sqrt{\frac{e^{2} N_{\mathrm{e}}}{\varepsilon_{0} m_{\mathrm{e}}}},
$$

which is called the electron plasma frequency. When the non-infinite ion mass $m_{\mathrm{i}}$ is taken into account, the oscillation frequency changes to

$$
\omega=\sqrt{\omega_{\mathrm{pe}}^{2}+\omega_{\mathrm{pi}}^{2}},
$$

with

$$
\omega_{\mathrm{pi}}=\sqrt{\frac{e^{2} N_{\mathrm{i}}}{\varepsilon_{0} m_{\mathrm{i}}}}
$$

the ion plasma frequency. In a low pressure argon plasma, with typical ion and electron densities of $10^{16} \mathrm{~m}^{-3}$, the electron and ion plasma frequencies are $900 \mathrm{MHz}$ and $3.31 \mathrm{MHz}$, respectively. This shows that the electrons can easily follow the typical electric field of $13.56 \mathrm{MHz}$, whereas ions cannot.

For charged particles in a plasma, the momentum balance equation can be reduced to the so-called drift-diffusion equation $[29,31]$ :

$$
\Gamma_{\mathrm{e}, \mathrm{i}}= \pm \mu_{\mathrm{e}, \mathrm{i}} N_{\mathrm{e}, \mathrm{i}} E-D_{\mathrm{e}, \mathrm{i}} \nabla N_{\mathrm{e}, \mathrm{i}}
$$

where the plus or minus sign in the drift term depends on the charge of the particle, $E$ is the local electric field, and $\mu_{\mathrm{e}, \mathrm{i}}$ and $D_{\mathrm{e}, \mathrm{i}}$ are the macroscopic mobility and diffusion constants of the electrons or ions, respectively. The mobility,

$$
\mu=\frac{|q|}{m v_{\mathrm{m}}},
$$


depends on the electric charge $q$ and the momentum transfer collision frequency $v_{\mathrm{m}}$. The diffusion coefficient $D$ can be related to the particle temperature through the Einstein relation:

$$
\frac{D}{\mu}=\frac{k T}{e} .
$$

In a stationary plasma, besides the densities, also the fluxes of ions and electrons must be equal. Evaluating the drift-diffusion equation results in

$$
\Gamma=-\frac{\mu_{\mathrm{e}} D_{\mathrm{i}}+\mu_{\mathrm{i}} D_{\mathrm{e}}}{\mu_{\mathrm{i}}+\mu_{\mathrm{e}}} \nabla N=-D_{\mathrm{a}} \nabla N,
$$

with $D_{\mathrm{a}}$ the ambipolar diffusion coefficient. In the plasma treated here, $\mu_{\mathrm{e}} \gg \mu_{\mathrm{i}}$, so using equations (3.26) and (3.27):

$$
D_{\mathrm{a}} \approx D_{\mathrm{i}}\left(1+\frac{T_{\mathrm{e}}}{T_{\mathrm{i}}}\right) \approx \mu_{\mathrm{i}} \frac{k T_{\mathrm{e}}}{e} .
$$

This indicates that the ambipolar diffusion coefficient is in first approximation determined by the mobility of the ions and the temperature of the electrons.

\subsection{Particle collisions}

Collisions between particles are a means of exchanging energy until the volume of gas attains a steady mean energy. When the particles are atoms or molecules, the collision is visualized as that between hard billiard balls; the total kinetic energy is conserved and the trajectory of each particle will usually be different before and after collision. Such collisions are called elastic collisions. However, a collision between particles may also result in a change of the internal energy of one or both particles and such collisions are called inelastic collisions. Examples of these are dissociation, excitation, ionization, and recombination.

Successful theoretical analysis of discharges depends on detailed knowledge of the energy exchange mechanisms and the onset energies of each inelastic collision. We shall consider particle collisions briefly in the following section. The treatise of Lieberman and Lichtenberg [29] will be followed here.

When a beam of particles with velocity $v$ and density $N$ interacts with a group of particles in rest with density $N_{\mathrm{g}}$, only a fraction of the incoming particles will collide. The initial flux $\Gamma=N v$ changes with $\mathrm{d} \Gamma$ over a distance $\mathrm{d} x$ according to

$$
\mathrm{d} \Gamma=-\sigma(v) \Gamma N_{\mathrm{g}} \mathrm{d} x .
$$


The proportionality constant $\sigma$ is the cross section, which is determined by the collision process. When $\sigma$ is independent on particle velocity, equation (3.29) can be solved:

$$
\Gamma=\Gamma_{0} \exp \left(-\frac{x}{\lambda_{\mathrm{m}}}\right),
$$

where $\Gamma_{0}$ is the initial (uncollided) flux at $x=0$; the mean free path $\lambda_{\mathrm{m}}$ is given by

$$
\lambda_{\mathrm{m}}=\frac{1}{N_{\mathrm{g}} \sigma} \text {. }
$$

Often the mean time between collisions $\tau$ is used:

$$
\tau=\frac{\lambda_{\mathrm{m}}}{v}
$$

or the inverse:

$$
v=\frac{1}{\tau}=N_{\mathrm{g}} \sigma v
$$

which is the collision frequency. When this frequency is expressed per unit of particle density, the rate constant $k$ is obtained:

$$
k=\frac{v}{N_{g}}=\sigma v .
$$

The cross section is often a function of velocity for inelastic collisions. Then equation (3.34) must be replaced with the integration over the velocity distribution function $f_{v}(v)$ :

$$
k=\int \sigma(v) v f_{v}(v) \mathrm{d} v
$$

The cross sections of elastic collisions are almost independent of the velocity and related to the radii of the particles. Two particles, having radius $r$, collide with each other if their centers come within a distance of $2 r$. In principle, the cross section of elastic collision, $\sigma_{\mathrm{el}}$, is then defined as $4 \pi r^{2}\left(\mathrm{~m}^{2}\right)$ [32]. Since the radius of an argon atom is $1.8 \cdot 10^{-10} \mathrm{~m}, \sigma_{\mathrm{el}}$ is approximately equal to $4 \cdot 10^{-19} \mathrm{~m}^{2}$ for elastic argon-argon collisions, fairly close to the measured value of $\sigma_{\mathrm{el}}=5.6 \cdot 10^{-19} \mathrm{~m}^{2}$ [33]. The cross section of elastic electron-argon collisions is about $2.5 \cdot 10^{-19} \mathrm{~m}^{2}$ [34]. At a typical pressure range of $1-10 \mathrm{~Pa}$ and $300 \mathrm{~K}$, the number of molecules in a gas is $2.4 \cdot 10^{20}-2.4 \cdot 10^{21} \mathrm{~m}^{-3}$, so the mean free path of argon atoms is $0.7-7 \mathrm{~mm}$ and of electrons $1.7-17 \mathrm{~mm}$ in between elastic collisions (equation (3.31)). 


\subsection{Evaluation of criteria and domains}

It is now possible to show that our standard argon discharge at typical HDRP conditions ( $c f$. Table 1.2) fulfills the basic requirements of quasi-neutrality. One can also study electron production processes and the various loss processes on forehand. An evaluation of these processes relies on a calculation of the characteristic electron temperature as a function of pressure, which is given in this section. It will be shown that recombination processes within the plasma volume can be ignored at typical process conditions. Thus, particles generated in the plasma will eventually reach the walls and the substrate and transfer their energies to them. An impression of the order of magnitude of the effects involved will be given in section 3.4 and will later be used in section 4.1.6.

An essential condition to achieve quasi-neutrality is that the dimensions of the plasma (or reactor) are much larger than the shielding distance (see below equation (3.17)), that is

$$
l \gg \lambda_{\mathrm{D}},
$$

where $l$ is the smallest dimension of the system. This criterion is easily obeyed since we calculated $\lambda_{\mathrm{D}}$ to be $0.1 \mathrm{~mm}$ (eq. (3.17)), and therefore $N_{\mathrm{e}}=N_{\mathrm{i}}$ and one speaks of plasma density, dispensing with the electron and ion densities. However, a velocity distribution function was used (equation (3.13)), and because of that enough charged particles should be present in this shielding distance or it would not have been a statistically sound concept. The crucial parameter here is the number of particles in a Debye sphere $N_{\mathrm{D}}$. This number must be larger than unity,

$$
N_{\mathrm{D}}=\frac{4}{3} \pi N_{\mathrm{i}} \lambda_{\mathrm{D}}^{3} \gg 1
$$

$N_{\mathrm{D}}$ amounts to $4 \cdot 10^{5}$ with a plasma density $N_{\mathrm{i}}$ of $10^{16} \mathrm{~m}^{-3}$.

The fact that charged particles behave collectively $[16,35]$ means that plasmas can support a wide variety of wave motions and oscillations. We noticed earlier that one such basic oscillation, the electron plasma frequency, $\omega_{\text {pe, }}$ arose if a group of electrons was slightly displaced with respect to the ions under influence of electromagnetic forces. Electromagnetic interactions dominate over the processes of ordinary gas kinetics when the electron plasma frequency (plasma oscillations of electrons) is high compared to the electron-neutral collision frequency (i.e., frequency of collisions between electrons and neutral particles). The elastic electron-neutral collision frequency, $v_{e}$, can be evaluated to be $57 \mathrm{MHz}$ for a typical plasma with a characteristic electron temperature of $2 \mathrm{eV}$ $\left(v_{\mathrm{e}}=9.5 \cdot 10^{5} \mathrm{~m} / \mathrm{s}\right.$, see equation (3.3)), and a pressure of $1 \mathrm{~Pa}\left(N_{\mathrm{g}}=2.4 \cdot 10^{20}\right.$ 
$\mathrm{m}^{-3}$ and $\sigma_{\mathrm{el}}=2.5 \cdot 10^{-19} \mathrm{~m}^{2}$, see equation (3.35)). This value is indeed much lower than the electron plasma frequency, $900 \mathrm{MHz}$, which was calculated for the same conditions (equation (3.21)). At $10 \mathrm{~Pa}$, however, the electron-neutral collision frequency approaches the plasma frequency. This means a loss of forward momentum during an electron oscillation and hence the momentum balance (equation (3.27)) is influenced. Furthermore, electrons will lose more energy per time interval due to the larger number of elastic collisions.

This decrease in electron temperature at higher pressures can also be calculated from an evaluation of the ion-electron production and loss processes $[36,37]$. We will assume a characteristic electron temperature of $k T_{\mathrm{e}}=2 \mathrm{eV}$ and $N_{\mathrm{e}}=N_{\mathrm{i}}=10^{16} \mathrm{~m}^{3}$ at a pressure of $1 \mathrm{~Pa}$, and will calculate the electron temperature at $10 \mathrm{~Pa}$.

The total production rate $S\left(\mathrm{~s}^{-1}\right)$ of ions and electrons is

$$
S=k_{\mathrm{iz}} N_{\mathrm{g}} N_{\mathrm{e}} V
$$

with $k_{\mathrm{iz}}$ the ionization rate constant, $N_{\mathrm{g}}$ the gas density, $N_{\mathrm{e}}$ the electron density and $V$ the volume of the plasma.

The loss mechanisms of charge particles are ambipolar flux to the walls and recombination. The rate constant for radiative recombination:

$$
\mathrm{Ar}^{+}+\mathrm{e}^{-} \rightarrow \mathrm{Ar}+h v
$$

$k_{\text {rec }}$, is $10^{-19} \mathrm{~m}^{3} \mathrm{~s}^{-1}$ (for an electron temperature of $2 \mathrm{eV}$ [38]). The radiative recombination rate for our typical ion density of $10^{16} \mathrm{~m}^{3}$ and a volume of 68 liters is $R_{\text {rec }}=k_{\text {rec }} N_{\mathrm{e}}^{2} V=10^{12} \mathrm{~s}^{-1}$.

The rate constant for three-body recombination:

$$
\mathrm{Ar}^{+}+2 \mathrm{e}^{-} \rightarrow \mathrm{Ar}+\mathrm{e}^{-}
$$

is in the order of $10^{-24} \mathrm{~m}^{3} \mathrm{~s}^{-1}$ (for $k T_{\mathrm{e}}=2 \mathrm{eV}$ and $N_{\mathrm{e}}=10^{16} \mathrm{~m}^{3}$ ) and can be neglected [29]. The ambipolar diffusion is typically determined by the flux of charged particles to the walls, having the Bohm velocity (equation (3.9)). The ambipolar loss rate is given by

$$
R_{\mathrm{amb}}=0.6 N_{\mathrm{e}} v_{\mathrm{B}} A,
$$

where $A$ is the reactor wall surface area. For $v_{B}=2.2 \cdot 10^{3} \mathrm{~ms}^{-1}$, a plasma density of $10^{16} \mathrm{~m}^{-3}$, and a wall surface of $1 \mathrm{~m}^{2}, R_{\mathrm{amb}}=2 \cdot 10^{19} \mathrm{~s}^{-1}$. Therefore, the contribution of recombination to the total loss of charged particles can be neglected. 
The loss equals the production in steady state, so after combining equations (3.38) and (3.39):

$$
\frac{k_{\mathrm{iz}}}{v_{\mathrm{B}}}=\frac{0.6 \mathrm{~A}}{N_{\mathrm{g}} V},
$$

The left-hand side of equation (3.40) depends only on electron temperature, whereas the right-hand side depends only on gas pressure. Therefore, the electron temperature can be solved as a function of pressure when $k_{\mathrm{iz}}$ is calculated by integration of the total ionization cross section [32] over the velocity distribution function (equation (3.35)), and equation (3.9) is used for $v_{\mathrm{B}}$. If a Maxwellian distribution with a characteristic electron temperature of $\mathbf{2} \mathbf{~ e V}$ is assumed at a pressure of $1 \mathrm{~Pa}$, one obtains an electron temperature of $\mathbf{1 . 6} \mathbf{e V}$ at $10 \mathrm{~Pa}$, using equation (3.40). Other authors also found this relation of electron temperature with total pressure [39].

\subsection{Energy transfer}

The energy dissipation in the plasma reactor is estimated in this section. It is assumed that all absorbed electromagnetic power is transferred to the electrons, which is reasonable, because of the high mass of the ions (see equation (3.23)). Particles generated in the plasma will eventually reach the walls and the substrate and transfer their energies to them. An impression of the order of magnitude of the effects involved will be obtained by considering a standard argon discharge. The global model of Lieberman and Gottscho [22] is extended to do so.

The energy of the electrons is lost by several processes:

1. Elastic collisions with atoms: The transferred energy per elastic collision is given by [32]

$$
E_{\mathrm{el}}=\frac{2 m_{\mathrm{Ar}} m_{\mathrm{e}}}{\left(m_{\mathrm{e}}+m_{\mathrm{Ar}}\right)^{2}} \frac{3}{2} k T_{\mathrm{e}} \cong \frac{2 m_{\mathrm{e}}}{m_{\mathrm{Ar}}} \frac{3}{2} k T_{\mathrm{e}},
$$

where $m_{\mathrm{Ar}}$ is the argon mass. The corresponding energy loss per second is given by

$$
P_{\mathrm{el}}=N_{\mathrm{e}} N_{\mathrm{g}} V k_{\mathrm{el}} \frac{3 m_{\mathrm{e}}}{m_{\mathrm{Ar}}} k T_{\mathrm{e}},
$$

where $k_{\mathrm{el}}$ is the rate constant for elastic electron-neutral collisions. 
2. Excitation: the energy loss $E_{\text {exc }}$ is at least the difference between the ground state and the first (4s) excited state of argon $(11.56 \mathrm{eV})$, and the corresponding energy loss per second is given by

$$
P_{\text {exc }}=N_{\mathrm{e}} N_{\mathrm{g}} V k_{\mathrm{exc}} E_{\mathrm{exc}} \text {. }
$$

3. Ionization: the energy loss $E_{\mathrm{iz}}$ is at least the ionization energy of $15.8 \mathrm{eV}$, and hence:

$$
P_{\mathrm{iz}}=N_{\mathrm{e}} N_{\mathrm{g}} V k_{\mathrm{iz}} E_{\mathrm{iz}} .
$$

The appropriate $k_{\mathrm{exc}}$ and $k_{\mathrm{iz}}$ can be calculated by integrating the corresponding total excitation and ionization cross sections [32] over the Maxwellian electron energy distribution function (equation (3.35)), while $k_{\text {el }}$ can be obtained from equation (3.34). Recombination is neglected as argued before.

4. Kinetic loss from ions and electrons escaping to the wall. When an ion is lost to a floating wall, its kinetic energy is determined by the difference between the plasma and floating potential, see equation (3.11), and by the acceleration in the presheath to $\frac{1}{2} k T_{\mathrm{e}}$, and measures: $\alpha k T_{\mathrm{e}}$; with $\alpha=4.7+0.5=5.2$ for argon. Ions are also neutralized upon collision with walls and hence lose at least their ionization energy of $15.8 \mathrm{eV}$ [40]. A neutralization probability of one is assumed. The electrons lose energies of $2 k T_{\mathrm{e}}$ when they are absorbed by the wall [29]. This value can be obtained by integration of the Maxwellian distribution. This value is higher than the average energy, because only the fast electrons are lost and the slow electrons reflect in the sheath. The energy loss to the walls by ions and electrons is given by

$$
\begin{aligned}
& P_{\text {wall,ions }}=0.6 N_{\mathrm{i}} v_{\mathrm{B}} A\left(\alpha k T_{\mathrm{e}}+E_{\mathrm{iz}}\right), \\
& P_{\text {wall,electrons }}=0.6 N_{\mathrm{e}} v_{\mathrm{B}} A 2 k T_{\mathrm{e}} .
\end{aligned}
$$

Two other mechanisms of energy dissipation are considered:

5. Relaxation of excited argon atoms by wall collisions [41]. The energy loss is at least $11.56 \mathrm{eV}$ and is determined by the thermal flux to the wall, hence

$$
P_{\text {wall,Ar* }}=\frac{1}{4} N_{\mathrm{Ar}^{*}}\left\langle v_{\mathrm{Ar}^{*}}\right\rangle A E_{\mathrm{exc}},
$$

where $N_{\text {Ar* }}$ is the density of excited argon atoms, and wherein the average thermal velocity, $\left\langle v_{\mathrm{Ar}^{*}}\right\rangle$, is determined by equation (3.3). Again, a relaxation probability of one is assumed. 
6. Radiative relaxation. Excited argon atoms will spontaneously return to the ground state in one or several transitions. Each transition is accompanied by the emission of a photon of a specific energy, equal to the energy difference between the quantum levels. Unfortunately, the calculation of absolute photon fluxes in plasmas is a complicated task [42,43], and there are virtually no measurements of absolute photon fluxes in high-density plasmas available [22]. On the other hand, relaxation is just the inverse of excitation, and therefore their powers should equal:

$$
P_{\text {exc }}=P_{\text {relaxation }}
$$

Several relaxation processes can occur: radiative relaxation, $P_{\text {rad }}$, relaxation at the reactor walls, $P_{\text {wall,Ar* }}$ (described above), and relaxation of metastable argon atoms, $P_{\text {metastable. Inserting these processes in equation }}$ (3.48) yields:

$$
P_{\text {exc }}=P_{\text {rad }}+P_{\text {wall,Ar* }}+P_{\text {metastable }} .
$$

The latter two processes are nonradiative [44-46]. The power for nonradiative relaxation of metastable argon atoms is roughly one fifth of the power for radiative relaxation, based on the ratio of the cross sections for the formation of metastables to the total excitation cross section [32]. Other sources of radiation in a plasma include radiative recombination and radiative transitions between different $\mathrm{Ar}^{+}$levels. However, the rate constant for radiative recombination was calculated above to be insignificant. Radiative transitions between $\mathrm{Ar}^{+}$levels do appear but densities and hence peak intensities are typically much lower than intensities of excited argon levels [47-49]. Thus, radiative relaxation is the main source of radiation, and $P_{\text {rad }}$ can be calculated from equation (3.49).

The various energy losses are presented in Table 3.1 for two electron densities at a argon pressure of $1 \mathrm{~Pa}$ and a characteristic temperature of $2 \mathrm{eV}$, and for a pressure of $10 \mathrm{~Pa}$ and $k T_{\mathrm{e}}=1.6 \mathrm{eV}$ (see equation (3.40)). One can observe from Table 3.1 that most of the absorbed energy is lost to the walls. One can observe also that $3-7 \%$ of the energy is transferred into radiation. The calculated values were confirmed by optical emission measurements in the spectral range of 250-1100 nm (see section 4.3.5 for details. For example, while $P_{\text {rad }}$ was calculated to measure $7 \mathrm{~W}\left(\right.$ at $\left.1 \mathrm{~Pa}, N_{\mathrm{e}}=1 \cdot 10^{16} \mathrm{~m}^{-3}, k T_{\mathrm{e}}=2 \mathrm{eV}\right)$, the measured value was $8.3 \mathrm{~W}$ (at approx. the same process conditions). 
Table 3.1: Energy losses of several typical argon plasmas.

$V=68 \mathrm{I}, A=1 \mathrm{~m}^{2},\left\langle v_{\text {th }, A r^{*}}\right\rangle=400 \mathrm{~m} / \mathrm{s}$ (eq. (3.3));

at $1 \mathrm{~Pa}: N_{\mathrm{g}}=2.4 \cdot 10^{20} \mathrm{~m}^{-3}, k_{\mathrm{el}}=2.4 \cdot 10^{-13} \mathrm{~m}^{3} \mathrm{~s}^{-1}$ (eq. (3.34)), $k_{\mathrm{iz}}=1.4 \cdot 10^{-17}$ $\mathrm{m}^{3} \mathrm{~s}^{-1}$ [32], $k_{\text {exc }}=6.4 \cdot 10^{-17} \mathrm{~m}^{3} \mathrm{~s}^{-1}$ [32];

at $10 \mathrm{~Pa}: N_{\mathrm{g}}=2.4 \cdot 10^{21} \mathrm{~m}^{-3}, k_{\mathrm{el}}=2.1 \cdot 10^{-13} \mathrm{~m}^{3} \mathrm{~s}^{-1}$ (eq. (3.34)), $k_{\mathrm{iz}}=1.2 \cdot 10^{-18}$ $\mathrm{m}^{3} \mathrm{~s}^{-1}$ [32], $k_{\text {exc }}=8.5 \cdot 10^{-18} \mathrm{~m}^{3} \mathrm{~s}^{-1}$ [32].

\begin{tabular}{llll}
\hline process conditions: & & & \\
\hline$p$ & $(\mathrm{~Pa})$ & 1 & 10 \\
$N_{\mathrm{e}}$ & $\left(\mathrm{m}^{-3}\right)$ & $10^{16}$ & $10^{16}$ \\
$k T_{\mathrm{e}}$ & $(\mathrm{eV})$ & 2.0 & 1.6 \\
\hline calculated values: & & & \\
\hline$P_{\text {el }}$ & $(\mathrm{W})$ & 0.5 & 3.5 \\
$P_{\text {exc }}$ & $(\mathrm{W})$ & 19 & 26 \\
$P_{\text {iz }}$ & $(\mathrm{W})$ & 6 & 5 \\
$P_{\text {wall,ions }}$ & $(\mathrm{W})$ & 55 & 44 \\
$P_{\text {wall,electrons }}$ & $(\mathrm{W})$ & 8 & 6 \\
$P_{\text {wall,Ar* }}$ & $(\mathrm{W})$ & 8 & 13 \\
$P_{\text {rad }}$ & $(\mathrm{W})$ & 7 & 8 \\
$P_{\text {total }}$ & $(\mathrm{W})$ & 103.5 & 105.5 \\
\hline
\end{tabular}




\section{Experimental equipment}

\subsection{ICPECVD system}

Figure 4.1 schematically shows the system set-up. The inductively coupled plasma source (supplied by Alcatel Micro Machining Systems; $13.56 \mathrm{MHz}$, max. electric power of $2 \mathrm{~kW}$ ) is placed on top of the chamber (also supplied by Alcatel and commercially available as an Adixen "I-Speeder" AMS 200). Quoted electric powers are measured at the output of the rf generator (RF power products, model FR20S), and are not corrected for losses.

The wafer is placed in the load lock on a molybdenum susceptor. After a pump-down cycle, the wafer is transferred into the chamber by an automated arm, and positioned on the chuck. The chuck is situated at the bottom of this chamber. The chuck can be heated up to $400^{\circ} \mathrm{C}$, but the targeted deposition temperatures should not exceed $150^{\circ} \mathrm{C}$. The temperature is controlled by a PID (proportional-integral-derivative) controller via a thermo-couple inserted in the chuck. The wafer is mechanically clamped to the chuck. Argon backing pressure of $1 \mathrm{kPa}$ (10 mbar) between both the susceptor and the chuck, and between the wafer and the susceptor, guarantees a good heat transfer between the chuck and the wafer $[50,51]$, see section 4.1 .6 for more details. The chuck can be rf-biased (ENI 13.56 MHz rf generator ACG-3b) and can be moved upwards into the chamber, i.e., towards the plasma source. Furthermore, in Figure 4.1, two gas inlets are shown: one above the plasma source and one below, in the chamber. An extensive gas distribution system (not shown in the figure) will supply gases to the deposition system, optionally in a pulsed mode. Mass flow controllers (Bronkhorst High-Tech B.V.) are used to control the gas flows. The 


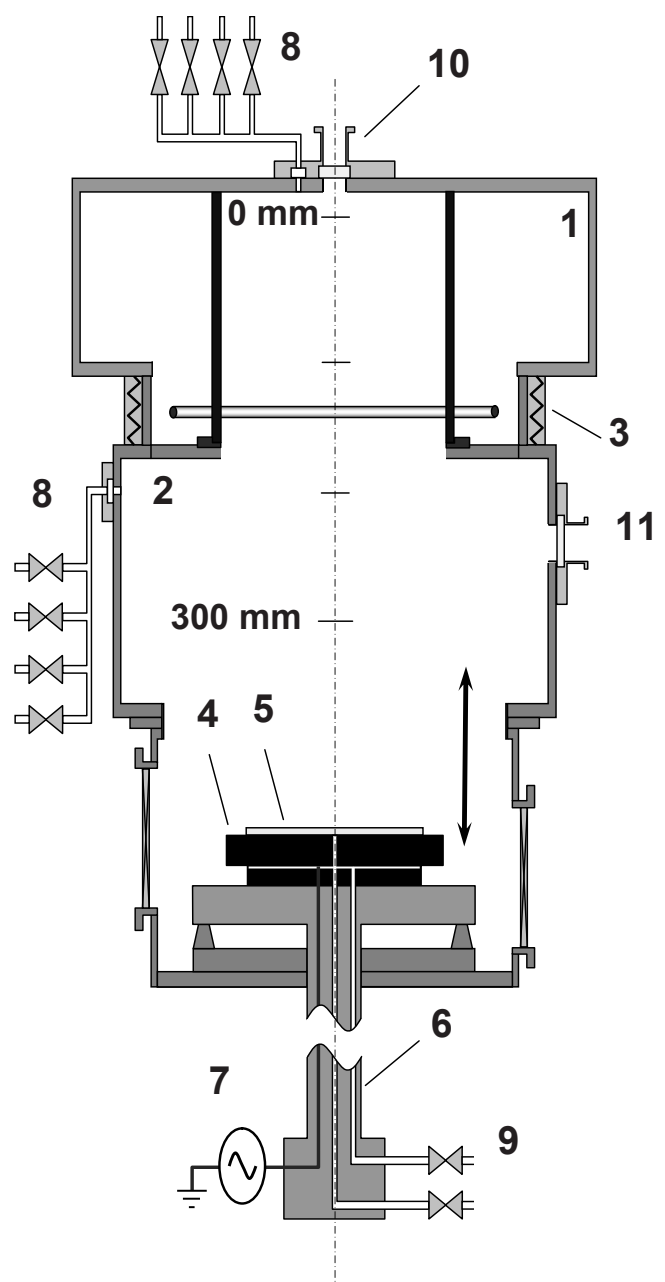

Figure 4.1: A schematic drawing of the remote-plasma CVD system: (1) ICP source, (2) chamber, (3) dc coil to introduce axial magnetic field, (4) chuck and susceptor, (5) wafer, (6) motor-driven arm, (7) rf-source for chuck biasing, (8) gas-inlets, (9) gas-inlet to control pressure between wafer, susceptor and chuck, (10) port to introduce Langmuir probe, (11) quartz viewport; the gas distribution system, load lock and vacuum pumps are not shown. 
system is evacuated with an oil-free turbo molecular pump (Adixen ATH 1300 $\mathrm{M}$, effective argon pumping speed $800 \mathrm{l} / \mathrm{min}$ ) backed by a dry pump (Adixen ACP $40 \mathrm{G})$; base pressure is $5 \cdot 10^{-5} \mathrm{~Pa}\left(5 \cdot 10^{-7} \mathrm{mbar}\right)$ or lower. A set of Pfeiffer capacitance gauges are used to accurately measure the process pressures (Pfeiffer CMR 261 and CMR 263), whereas a combined cold wall and Pirani gauge (Pfeiffer PKR 261) measures from base pressure to atmospheric pressure.

Although the base pressure is $5 \cdot 10^{-5} \mathrm{~Pa}$, the pressure in the chamber rises to $8 \cdot 10^{-2} \mathrm{~Pa}$ as soon as the backing pressure between the wafer and chuck is set. This corresponds to an argon gas leakage into the chamber of $5 \mathrm{sccm}$, as has been obtained by pressure measurements as a function of calibrated gas flows.

To measure the volume of the chamber, the chamber was evacuated and the throttle valve between the chamber and the pump was closed. At time $t_{1}=0$ and pressure $p_{1}$ a known flow of argon gas $(20-50 \mathrm{sccm}$, equivalent to $0.254-0.635$ Torr $\cdot 1 \cdot \mathrm{s}^{-1}$ ) was allowed into the chamber and the resulting pressure $p_{2}$ was noted at time $t_{2}$. The volume of the chamber was calculated from quotient of the measured $\Delta t=t_{2}-t_{1}$ and $\Delta p=p_{2}-p_{1}$, multiplied by the gas flow. The volume thus obtained measured 68 liters.

\subsubsection{The variable axial magnetic field}

The ICP-source is also equipped with an external dc coil (item (3) in Figure 4.1, see also Figure 4.2 for a more detailed view) which can induce an axial magnetic field $B$. Combination with the radial electric field $E_{\mathrm{rf}}$ of the ICP-source will then cause an oscillatory azimuthal motion of the plasma electrons (the well-known $E \times B$ drift). Due to the Lorentz force, the length of the electron trajectory is increased, which results in more collisions and better ionization and power absorption efficiency [33]. Increases of electron densities by a factor of ten or more have been reported $[52,53]$. Since the ions do not move appreciably on the time scale of the rf period, they do not exhibit this azimuthal motion. The maximum magnetic flux density that can be applied (by setting the current through the coil) is $9.5 \mu \mathrm{T}$.

\subsubsection{The magnetic multipole wall}

The use of permanent magnets to confine the charged particles in a plasma has found a variety of applications $[54,55]$. The principle idea is that a local magnetic field, parallel and close to the plasma boundary, will reflect charge particles moving outwards. Such fields can be generated by placing permanent magnets with their north and south poles in alternating order along the discharge confining walls, see Figure 4.3 . Such arrangements are generally called 


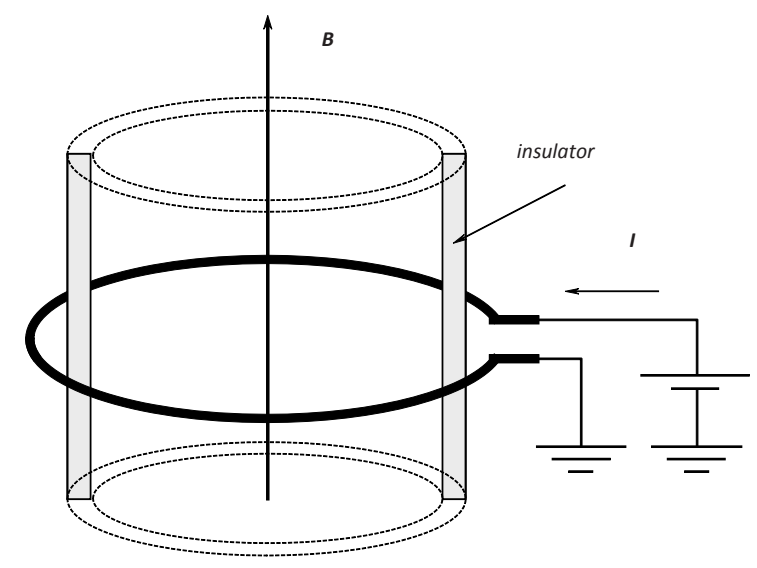

Figure 4.2: dc coil to introduce axial magnetic field

cusp shaped or multipole fields. Different field configurations have been employed, like checkerboard and line cusp geometries [56]. The improvement of density and homogeneity of plasma by this technique are well established [57]. The latter was in fact the reason why these magnets were mounted to the reactor chamber by Alcatel [58]. The outer chamber wall has been equipped with a line cusp arrangement, consisting of 14 vertical rows of magnets parallel to the vertical chamber axis.

\subsubsection{Impedance matching networks}

If the antenna were directly connected to the $13.56 \mathrm{MHz}$ rf power source, the power transfer from the source to antenna would be inefficient because of impedance mismatch $[59,60]$. Preferably, the plasma has to absorb all of the power transmitted from the source. To achieve this, the source impedance must be equal to the conjugate of the (complex) plasma load impedance. The impedance of an antenna is sensitive to plasma changes and always becomes complex [60]. To eliminate the imaginary part of the plasma load, to obtain the characteristic impedance of $50 \Omega$, the matching network should have an output impedance which equals the conjugate of the antenna impedance. Tunable capacitors can re-tune the circuit's impedance towards or to the characteristic impedance. Two commercially available automated matching networks are used in this set-up (an ENI MWH-5-01 between the chuck bias source and antenna, and a Sairem CBA PL/M between ICP source and antenna) comprised 


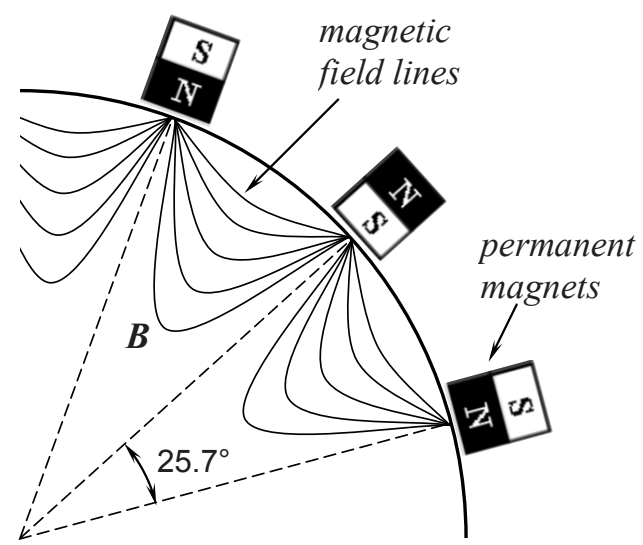

Figure 4.3: Permanent magnets mounted on the outside of the reactor chamber wall.

of tuning units and a control unit. The tuning units contain the matching components (fixed inductor and variable capacitors), two dc motor servos, and rf sensors to provide feedback to the dc motor servos. The control unit provides controls for manual and remote operation of the tuning unit.

\subsubsection{External oscillator}

The frequencies of our rf power sources are controlled by quartz crystal oscillators operating at a resonance frequency of $13.56 \mathrm{MHz}$. Small-frequency oscillations (a few hertz) could be observed in our plasma when both the ICP-source and the chuck bias were operated at the same time. This was caused by small differences in the resonance frequencies of the quartz crystal oscillators of the two rf power sources. This can be explained by the interference of the two electromagnetic waves. Consider two electromagnetic waves $E_{1}$ and $E_{2}$ propagating outwards from the sources 1 and 2 and creating an oscillating electric field with amplitude $E$ :

$$
E(x, t)=E_{1}(x, t)+E_{2}(x, t)=E_{1} \sin \left(k_{1} x-\omega_{1} t\right)+E_{2} \sin \left(k_{2} x-\omega_{2} t\right),
$$

where $k_{1,2}$ and $\omega_{1,2}$ are the wave numbers and angular frequencies of the waves of sources 1 and 2, respectively. Applying the trigonometric prosthaphaeresis 


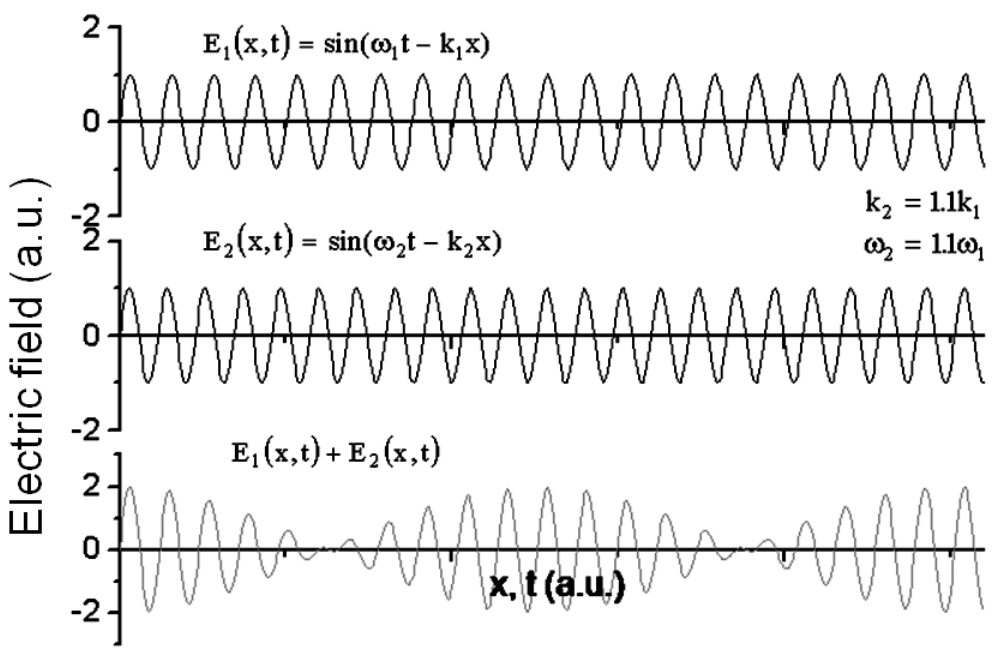

Figure 4.4: Two electromagnetic waves and their interference signal.

relation $^{1} \sin \alpha+\sin \beta=2 \sin \left(\frac{\alpha+\beta}{2}\right) \cos \left(\frac{\alpha-\beta}{2}\right)$ gives

$$
\begin{aligned}
& E(x, t)=2\left(E_{1}+E_{2}\right) \sin \left[\frac{1}{2}\left(k_{1}+k_{2}\right)\right.\left.x-\frac{1}{2}\left(\omega_{1}+\omega_{2}\right) t\right] \\
& \times \cos \left[\frac{1}{2}\left(k_{1}-k_{2}\right) x-\frac{1}{2}\left(\omega_{1}-\omega_{2}\right) t\right] .
\end{aligned}
$$

When analyzing equation (4.2) in the frequency-domain, one can observe that using two sources with different frequencies results in an electric field with an angular frequency $\omega_{+}=\frac{1}{2}\left(\omega_{1}+\omega_{2}\right)$, superimposed on an oscillation with a frequency $\omega_{-}=\frac{1}{2}\left(\omega_{1}-\omega_{2}\right)$. This is sketched in Figure 4.4 for a (hypothetical) situation where $\omega_{2}=1.1 \cdot \omega_{1}$. A frequency difference of $\leq 10 \mathrm{~Hz}$ between the two $13.56 \mathrm{MHz}$ sources would be more typical for our situation (but not easy to show in a figure). Such frequency differences are much lower than the ion plasma frequency (equation (3.23)) and will therefore influence ambipolar diffusion, floating potentials, and the dc-bias on the wafer $(10 \mathrm{~Hz}$ is considered dc here). One can synchronize both sources to avoid this.

1. Mathematicians consider this as a valid but otherwise truly obscure product-sum relation and it could just as well be forgotten... until needed by us! (According to David E. Joyce, Professor of Mathematics and Computer Science, Clark University, on http://www.clarku.edu/ djoyce/trig/identities.html.) 
An external master oscillator was used to synchronize both sources (Hüttinger Elektronik MOP2/13). This oscillator has the additional advantage that the phase of one of the two sources can be controlled as well. Two electromagnetic waves $E_{1}$ and $E_{2}$ with one angular frequency $\omega_{0}$ create an oscillating electric field with amplitude $E$ :

$$
E(x, t)=E_{1}(x, t)+E_{2}(x, t)=E_{1} \sin \left(k_{0} x-\omega_{0} t\right)+E_{2} \sin \left(k_{0} x-\omega_{0} t+\Phi\right),
$$

where $\Phi$ is the phase constant as set by the phase shifter of the master oscillator. Applying the trigonometric relation used in equation (4.2) also to equation (4.3) gives

$$
E(x, t)=2\left(E_{1}+E_{2}\right) \sin \left(k_{0} x-\omega_{0} t+\frac{1}{2} \Phi\right) \cos \left(\frac{1}{2} \Phi\right) .
$$

Equation (4.4) maximizes when $\Phi=0$, and minimizes for $\Phi=180^{\circ}$.

\subsubsection{Typical plasma operation conditions}

Argon was used as a carrier gas, and the process pressure ranged from 1 to $20 \mathrm{~Pa}$, while the argon flow was between $100-500 \mathrm{sccm}$. The process pressure was controlled by a feedback loop to the throttle valve. Flows of 10 to $100 \mathrm{sccm}$ of precursor gases, such as nitrogen $\left(\mathrm{N}_{2}\right)$, nitrous oxide $\left(\mathrm{N}_{2} \mathrm{O}\right)$, and $\mathrm{SiH}_{4}$ or $\mathrm{Ar}-\mathrm{SiH}_{4}\left(2 \%\right.$ silane $\left(\mathrm{SiH}_{4}\right)$ in argon), were added to the argon carrier gas to study their effect on the electron energy distribution and electron density of the plasma. The Ar- $\mathrm{SiH}_{4}-\mathrm{N}_{2} \mathrm{O}$ mixtures were used to deposit silicon oxide. The electric power supplied to the plasma source (i.e. rf-coil) was between 100 and $1000 \mathrm{~W}$, but typically $300 \mathrm{~W}$ at deposition conditions.

\subsubsection{Wafer temperature control}

Deposition processes are a careful balance of absorption, desorption, surface migration, and chemical reactions. A change of wafer temperature can disturb this balance. It is therefore important to analyze by which mechanisms wafers gain and lose energy during plasma processing. Moreover, since the thermocouple measures the temperature of the chuck and not that of the wafer directly, it is important to estimate to what extent the measured temperature represents the actual wafer temperature. Let us consider a typical process scheme to introduce this issue. As stated earlier, argon-backing pressure of $1 \mathrm{kPa}$ should transfer heat from the heated chuck to the (initially cold) wafer. The wafer reaches an equilibrium temperature $T_{\mathrm{W}}$ with its surroundings after a certain 
period. The plasma is ignited and the energy flux from the plasma will transfer heat to the wafer. Furthermore, chemical reactions during the deposition of silicon dioxide will also heat the wafer due to their exothermic nature. We will estimate how these processes influence $T_{\mathrm{w}}$. The heat transport between wafer and chuck will first be addressed. The model of Hasper et al. [50] was adopted to do so. Afterwards, heat fluxes from plasma and deposition processes will be discussed.

The heat transport between wafer and chuck can be described by four mechanisms; these are radiation, gas conduction, gas convection, and solidsolid conduction. The heat transfer due to the gas flow can be neglected since the gas flow between the chuck and wafer measured only $5 \mathrm{sccm}$ (due to leakage). Typical chamber pressures and gas flows are also too low to cause significant cooling by gas convection [50]. Heat conduction through solid-solid contact is neglected as well since only small areas on the wafer's outer perimeter are mechanically clamped to the susceptor. Therefore, only heat transport by radiation and gas conduction are considered.

\subsubsection{Radiation}

The Stefan-Boltzmann law [61-63] gives the heat flux from a grey body by radiation:

$$
P_{\text {rad }}=\varepsilon \sigma_{\mathrm{B}} T^{4}
$$

where $\varepsilon$ is the emission coefficient, $\sigma_{\mathrm{B}}$ the Stefan-Boltzmann constant $\left(\sigma_{\mathrm{B}}=\right.$ $5.67 \cdot 10^{-8} \mathrm{~W} / \mathrm{m}^{2} \cdot \mathrm{K}^{4}$ ), and $T$ the temperature of the grey body. For two parallel surfaces of the same area facing each other (e.g. wafer and susceptor), the energy flux per unit area due to radiation from the hot (temperature $T_{1}$ and emission coefficient $\varepsilon_{1}$ ) to the cold surface (temperature $T_{2}$ and emission coefficient $\varepsilon_{2}$ ) can be calculated [50] as

$$
P_{\text {rad }}=\left(\frac{\varepsilon_{1} \varepsilon_{2}}{\varepsilon_{1}+\varepsilon_{2}-\varepsilon_{1} \varepsilon_{2}}\right) \sigma_{\mathrm{B}}\left(T_{1}^{4}-T_{2}^{4}\right) .
$$

The pre-factor between brackets on the right hand side of equation (4.6) is a result of the radiative coupling between the two parallel surfaces [50]. This equation can be used to determine the energy flux between the susceptor and the wafer, with $\varepsilon_{1}=0.1$ (molybdenum [64]) and $\varepsilon_{2}=0.3$ (silicon [65]). The heated wafer will lose heat by radiation to the chamber, according to

$$
P_{\text {loss rad }}=\varepsilon_{\mathrm{Si}} \sigma_{\mathrm{B}}\left(T_{\mathrm{W}}^{4}-T_{0}^{4}\right)
$$


where $\varepsilon_{\mathrm{Si}}$ is the emission coefficient of the wafer $\left(\varepsilon_{\mathrm{Si}}=0.3[65]\right)$, and $T_{\mathrm{W}}$ and $T_{0}$ the temperature of the wafer and the chamber, respectively. No radiative coupling between the chamber and the wafer is assumed [65]. In vacuum (i.e., no backing pressure!) the temperature of the wafer is only determined by radiation, and one can calculate the wafer temperature, since at thermal equilibrium:

$$
P_{\text {rad }}-P_{\text {loss rad }}=0 \text {. }
$$

Setting the temperature of the susceptor at $423 \mathrm{~K}\left(150^{\circ} \mathrm{C}\right)$, the temperature of the chamber at $300 \mathrm{~K}\left(23^{\circ} \mathrm{C}\right)$, one can calculate a steady-state wafer temperature of $340 \mathrm{~K}\left(67^{\circ} \mathrm{C}\right)$. This temperature would have been closer to the susceptor's temperature if a susceptor material (or coating) with a higher emission coefficient would have been chosen.

The heat transfer by gas particles has to be considered also, when a backing pressure between the wafer and susceptor is established.

\subsubsection{Gas conduction}

The second mechanism for heat transfer is gas conduction. The energy flux per unit area for two parallel surfaces at a distance $d$ can be described [66] by

$$
P_{\mathrm{c}}=\frac{k_{\mathrm{m}}\left(T_{1}-T_{2}\right)}{d+2 g},
$$

where $k_{\mathrm{m}}$ is the mean thermal conductivity over the considered temperature range $\left(k_{\mathrm{m}}=1.1 \cdot 10^{-2} \mathrm{~J} \cdot \mathrm{m}^{-1} \cdot \mathrm{s}^{-1} \cdot \mathrm{K}^{-1}\right.$ for argon between $\left.300-500 \mathrm{~K}[50,66]\right)$, $d$ is the distance between the susceptor and the wafer, and is estimated to be $100 \mu \mathrm{m}$, and coefficient $g$ is equal to

$$
g=\frac{2-\alpha}{\alpha} \frac{9 \gamma-5}{2(\gamma+1)} \lambda
$$

where $\alpha$ is the thermal accommodation coefficient, $\gamma$ is the ratio of specific heat at constant pressure to that at constant volume $\left(\gamma=\frac{5}{3}\right.$ for monoatomic gases [66]), and $\lambda$ is the molecular mean free path. The thermal accommodation coefficient $\alpha$ is defined as the fractional extent to which molecules that reflect on a solid surface have their mean energy adjusted towards the temperature of that surface. The transferred energy per elastic collision is related to the masses of the gas $\left(m_{\mathrm{g}}\right)$ and surface molecules $\left(m_{\mathrm{s}}\right)$ [67] (see equation (3.41), for a similar approach),

$$
\alpha=\frac{4 m_{\mathrm{g}} m_{\mathrm{s}}}{\left(m_{\mathrm{g}}+m_{\mathrm{s}}\right)^{2}} .
$$


This leads to $\alpha_{\mathrm{Si}-\mathrm{Ar}}=0.7$ for argon to silicon, and $\alpha_{\mathrm{Mo}-\mathrm{Ar}}=0.83$ for argon to molybdenum. The value of the accommodation coefficient for the two surfaces can be calculated [67] with

$$
\alpha=\frac{\alpha_{\mathrm{Si}-\mathrm{Ar}} \alpha_{\mathrm{Mo}-\mathrm{Ar}}}{\alpha_{\mathrm{Si}-\mathrm{Ar}}+\alpha_{\mathrm{Mo}-\mathrm{Ar}}-\alpha_{\mathrm{Si}-\mathrm{Ar}} \alpha_{\mathrm{Mo}-\mathrm{Ar}}} .
$$

The mean free path for argon atoms can be calculated by equation (3.31), and measures $\lambda=7 \mu \mathrm{m}$ at $1 \mathrm{kPa}$ (the pressure between the susceptor and the wafer) and $0.7-7 \mathrm{~mm}$ at typical process pressures (1-10 Pa). The value of coefficient $g$ can now be calculated, and hence the heat transfer by gas conduction can be included in the calculation of the wafer temperature since at thermal equilibrium:

$$
P_{\text {rad }}+P_{\mathrm{c}}-P_{\text {loss rad }}=0 .
$$

Setting the temperature of the susceptor at $423 \mathrm{~K}\left(150^{\circ} \mathrm{C}\right)$, the temperature of the chamber at $300 \mathrm{~K}\left(23^{\circ} \mathrm{C}\right)$, and the backing pressure at $1 \mathrm{kPa}$ (at $d=100 \mu \mathrm{m})$, one can calculate a steady-state wafer temperature of $419 \mathrm{~K}$ $\left(146^{\circ} \mathrm{C}\right.$ ). For a susceptor temperature of $400^{\circ} \mathrm{C}$ (otherwise the same conditions), one will find a wafer temperature of $370^{\circ} \mathrm{C}$, comparable to measurements of Van Graven and Wolters [51].

Heat fluxes from plasma and deposition processes have to be addressed as well when a plasma is ignited.

\subsubsection{Heat fluxes from plasma}

Wafers gain energy by the energy flux from the plasma. Various energy fluxes from the plasma to the chamber walls were considered in section 3.4. They are summarized in Table 3.1 and Figure 4.5. These fluxes also apply to a wafer at floating potential and are repeated here. For a typical argon plasma at $1 \mathrm{~Pa}$, wafers gain energy by absorbing kinetic and potential energy of argon ions (55 $\left.\mathrm{W} / \mathrm{m}^{2}\right)$, kinetic energy of electrons $\left(8 \mathrm{~W} / \mathrm{m}^{2}\right)$, potential energy of excited argon atoms $\left(8 \mathrm{~W} / \mathrm{m}^{2}\right)$, and radiation from the plasma $\left(7 \mathrm{~W} / \mathrm{m}^{2}\right)$. A bare silicon wafer will reflect about $38 \%$ of the incoming radiation (at normal incidence, one can simplify the Fresnel reflection coefficients to

$$
R=\left(\frac{n_{\text {vacuum }}-n_{\mathrm{Si}}}{n_{\text {vacuum }}+n_{\mathrm{Si}}}\right)^{2},
$$

with $n$ as the refractive index, $n_{\text {vacuum }}=1$ and $n_{\mathrm{Si}}=4.2$ [68]). The growing film of silicon dioxide on top of the wafer will act as anti-reflective coating and 


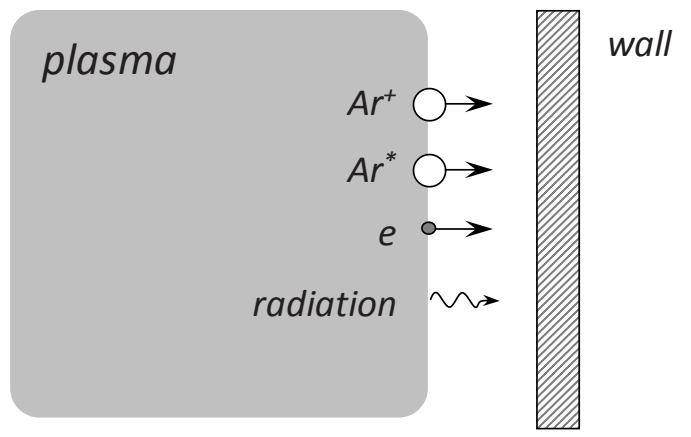

Figure 4.5: Heat fluxes towards a wall.

enhance absorption of radiation. In this thesis, the thickness of deposited silicon dioxide layers did typically not exceed $50 \mathrm{~nm}$, therefore the reflection decreased from $38 \%$ (at $0 \mathrm{~nm}$ ) to $24 \%$ (at $50 \mathrm{~nm}$ ) (calculated by using the Fresnel equations and a standard matrix calculation technique [69]). So, roughly $5 \mathrm{~W} / \mathrm{m}^{2}$ of the incoming radiation will be absorbed by the wafer. To conclude, a total energy flux of $P_{\text {plasma }}=76 \mathrm{~W} / \mathrm{m}^{2}$ is absorbed by the wafer due to the plasma processes at an argon pressure of $1 \mathrm{~Pa}$.

\subsubsection{Heat generation due to chemical reactions}

The wafer will also absorb energy because of the formation of silicon dioxide on its surface since this is an exothermic reaction. At constant pressure, the heatformation can be calculated from the reaction enthalpy $H_{\mathrm{f}}$ [70]. The enthalpy $H_{\mathrm{f}}$ for any reaction can be calculated by subtracting the enthalpies of formation of the reactants from those of the products $[70,71]$. In our case, silane $\left(\mathrm{SiH}_{4}\right)$ and nitrous oxide $\left(\mathrm{N}_{2} \mathrm{O}\right)$ are introduced in the plasma as the deposition precursors. As will be shown in section $6.4, \mathrm{Si}$ and $\mathrm{O}$ radicals are the dominant species from which silicon dioxide is formed:

$$
\mathrm{Si}(\mathrm{g})+2 \mathrm{O}(\mathrm{g}) \rightarrow \mathrm{SiO}_{2}(\mathrm{~s})
$$

and $H_{\mathrm{f}}=-1850 \mathrm{~kJ} / \mathrm{mol}$ and the reaction is exothermic (enthalpies of formation were found in $[64,71]$ ). This is equal to $H_{\mathrm{f}}=-70 \mathrm{~kJ} / \mathrm{cm}^{3}$ (with $\mathrm{SiO}_{2}$ mass density of $\rho=2.27 \mathrm{~g} / \mathrm{cm}^{3}$ and molar mass $M=60 \mathrm{~g} / \mathrm{mol}$ ). The power generated on the wafer depends on the deposition rate. If one assumes a deposition rate of $1 \mathrm{~nm} / \mathrm{s}$, then one can easily calculate that the formation of $\mathrm{SiO}_{2}$ adds a power of $P_{\text {depo }}=70 \mathrm{~W} / \mathrm{m}^{2}$ onto the wafer. 
Finally, the heat fluxes from the plasma and the heat exchange due to chemical reactions can be included in the calculation of the wafer temperature at thermal equilibrium:

$$
P_{\text {rad }}+P_{\mathrm{c}}+P_{\text {plasma }}+P_{\text {depo }}-P_{\text {loss rad }}=0 \text {. }
$$

At typical process conditions, this leads to a new equilibrium wafer temperature of $421 \mathrm{~K}\left(148^{\circ} \mathrm{C}\right)$. This temperature is only slightly higher than the one without plasma and deposition conditions $\left(146^{\circ} \mathrm{C}\right)$. This indicates that the wafer temperature is efficiently maintained during processing. Moreover, one can also calculate that one has to change plasma conditions or deposition rates drastically to influence the wafer temperature by e.g. more than $10^{\circ} \mathrm{C}$. One can conclude that the wafer temperature is stable for a wide range of process conditions, and the heat transfer between the wafer and the chuck is sufficient. This is maintained mainly by gas conduction (i.e., argon backing-pressure). We calculated earlier that if no backing pressure was applied (i.e., no gas conduction), the temperature of the wafer would be $67^{\circ} \mathrm{C}$. Applying plasma at deposition conditions would raise this equilibrium temperature by 42 degrees, i.e. up to $109^{\circ} \mathrm{C}$. Furthermore, the wafer temperature would be much more vulnerable to changes in process conditions without backing pressure (gas conduction).

We can conclude that the temperature control designed well and the measured chuck temperature represents the actual wafer temperature closely.

\subsection{Langmuir probe}

An rf-compensated Langmuir probe (Scientific Systems SmartProbe ${ }^{\mathrm{TM}}$ ) was used to determine the properties of the electrically charged particles in the plasma as function of position in the plasma. An exploded view of the Langmuir probe is shown in Figure 4.6. The active part of the Langmuir probe is a tungsten wire, $0.19 \mathrm{~mm}$ in diameter and $10 \mathrm{~mm}$ in length, which is inserted into the plasma. The tip is connected to an acquisition unit via a coaxial cable that is shielded from the plasma by an alumina shaft. The shaft length is 470 $\mathrm{mm}$ and its diameter is $9.5 \mathrm{~mm}$. The shaft is mounted onto a linear drive system so that the probe can be moved in vertical direction. The drive system is placed on top of the ICP source (see Figure 4.1). This system consists of vacuum bellows, a stepping motor, and a precision ball screw arrangement. Typically, probe current is measured at bias voltages ranging from -40 to $+40 \mathrm{~V}$. The drive system enables one to measure plasma parameters vertically through the plasma-generation zone, downwards to the chuck (i.e., at locations between 0 


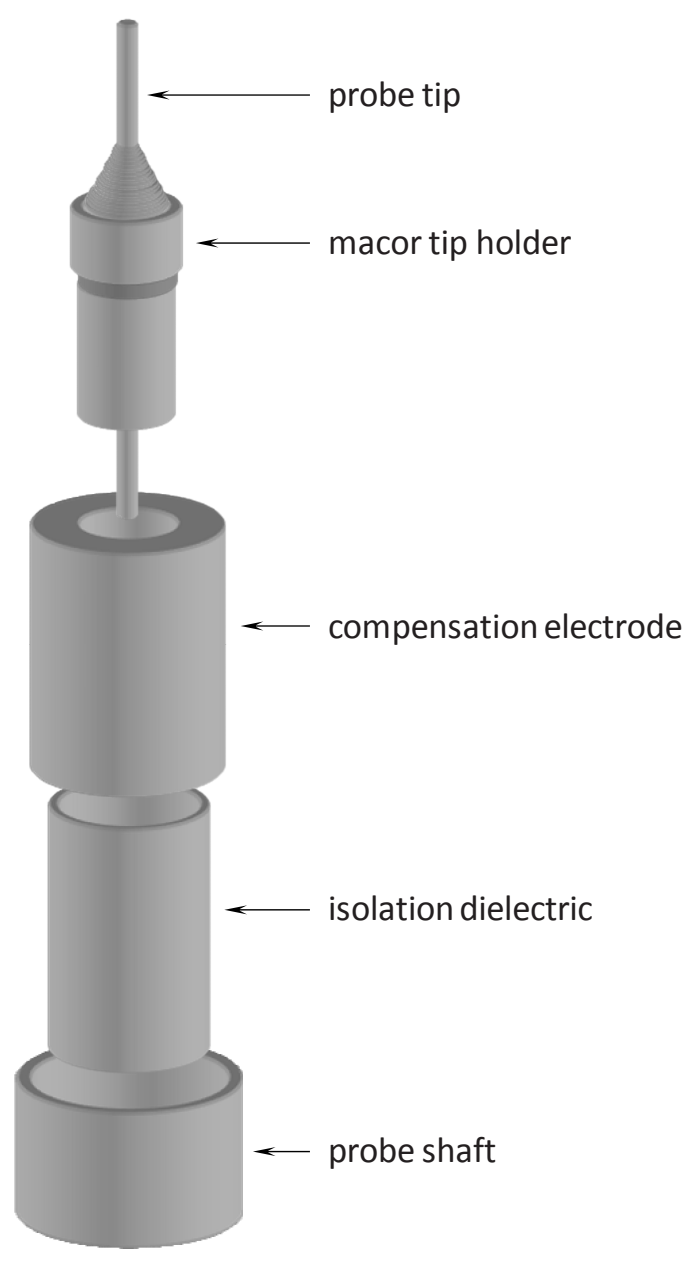

Figure 4.6: Exploded view of the Langmuir probe. 
and $300 \mathrm{~mm}$ on the vertical axis shown in Figure 4.1). However, the horizontal optical axis, used for monitoring the OES spectra, crosses the vertical axis at 240 $\mathrm{mm}$. Therefore, the presented OES data are compared with the Langmuir-probe data measured at this position.

The problems associated with Langmuir probes in $\mathrm{rf}$ plasmas are well known and described [72-77]. Hirsch [73] concluded that, for non-compensated probes, the apparent distribution of electrons (as measured by the probe) is a function of $\mathrm{rf}$ interactions in the probe sheath rather than electron energy distribution in plasma. Additionally, Paranjpe et al. [74] showed that the rf voltage across the probe-plasma sheath caused a time dependent variation of the plasma potential, which very much affected the measured electron energy distribution. The most common solution to minimize this $\mathrm{rf}$ distortion is provided by the manufacturers of this probe [76], and it involves increasing the probeto-ground impedance with respect to the plasma-to-probe-sheath impedance. This ensures that the rf voltage appears across the probe-to-ground impedance and not across the plasma-to-probe-sheath impedance. The probe-to-ground impedance is increased by introducing a pair of inductors that are self-resonant with the driving rf frequency of $13.56 \mathrm{MHz}$. The inductors are situated close to the probe tip to eliminate effects of stray capacitance [76]. A compensation electrode in contact with the plasma is capacitively coupled to the probe tip. The large capacitance and low impedance of this electrode reduces the probeto-plasma-sheath impedance [76].

A current, $I$, will be drawn from plasma when a dc bias, $V$, is applied to the probe. This will result in an $I-V$ curve as shown in Figure 4.7. The plasma potential $\left(V_{\mathrm{p}}\right)$ is defined as the voltage at the transition point between the electron retarding and the electron saturation parts of the curve, i.e., where the second derivative is zero $[78,79]\left(V_{p}=16.6 \mathrm{~V}\right.$ in Figure 4.7$)$. When the probe potential is lower than the plasma potential, only those electrons can be collected that have a thermal velocity satisfying $\frac{1}{2} m_{e} v_{\mathrm{e}}^{2}>\left(V_{\mathrm{p}}-V\right)$. If the ion current and electron current are equal in magnitude, we find a zero net current. The voltage at which this occurs is called the floating potential $\left(V_{\mathrm{f}}\right)$. Only positive ions are collected far below the floating potential, and we obtain the so-called ion saturation part of the probe $I-V$ curve. Above the plasma potential, only electrons are collected, and we measure the so-called electron saturation part of the probe $I-V$ curve. In practice, this current does not saturate (see Figure 4.7). The nonsaturation is explained by a current collected to a probe of increasing effective area, due to expansion of the sheath as a function of voltage [80]. Figure 4.7 also shows the curve as derived from Laframboise's theory [80-82]. One can observe from this figure that measurement and theory deviate especially 


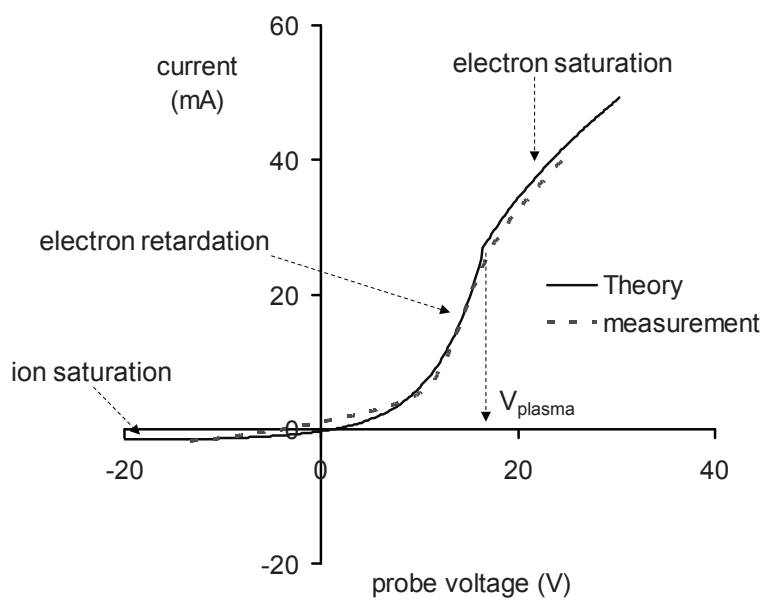

Figure 4.7: Typical Langmuir-probe measurement in our deposition system in argon plasma at $1 \mathrm{~Pa}$. The measured data were fitted according to Laframboise's theory.

in the electron retardation-part of the curve. The measured floating potential, for example, is more negative than the one obtained by theory. This will be explained in section 5.2.1.

Laframboise's theory was derived analogous to equations $(3.6,3.7,3.8$, $3.9,3.10,3.11$ ) in section 3 , under the assumption that the electron velocity distribution function is Maxwellian. From equation (3.6) the probe current, $I$, is found to be

$$
I=I_{\mathrm{e} 0} \exp \left(-\frac{e\left(V_{\mathrm{p}}-V\right)}{k T_{\mathrm{e}}}\right)-I_{\mathrm{i} 0}, \quad V<V_{\mathrm{p}},
$$

where $I_{\mathrm{e} 0}$ is the electron saturation current, and $I_{\mathrm{i} 0}$ is the ion saturation current. The electron saturation current (i.e., the current at $V=V_{\mathrm{p}}$ ) is determined by the thermal flux of the electrons, and can be obtained by substituting equation (3.3) in equation (3.5):

$$
I_{\mathrm{e} 0}=A e \frac{1}{4} N_{\mathrm{e}}\left\langle v_{\mathrm{e}}\right\rangle=A e N_{\mathrm{e}} \sqrt{\frac{k T_{\mathrm{e}}}{2 \pi m_{\mathrm{e}}}},
$$

where $A$ is the surface area of the probe. The ion saturation current is derived 
from equation (3.10):

$$
I_{\mathrm{i} 0} \approx 0.6 A N_{\mathrm{i}} v_{\mathrm{B}} \approx 0.6 A e N_{\mathrm{i}} \sqrt{\frac{k T_{\mathrm{e}}}{m_{\mathrm{Ar}}}} .
$$

The characteristic electron temperature $\left(k T_{\mathrm{e}}\right)$ can be either determined from equation (4.15) by plotting the slope of the $\ln (I)-V$ curve, or by substitution of the measured plasma and floating potential in equation (3.11). The $k T_{\mathrm{e}}$ thus found can be substituted in equations (4.16), (4.17) to calculate the electron and ion densities from the measured saturation currents.

However, a low-pressure discharge often has an electron energy distribution function (EEDF) that departs from a Maxwellian. The EEDF can be described more accurately by a two-temperature Maxwellian (or bi-Maxwellian) [83-85] at certain conditions, while Druyvesteyn and Penning showed that the energy distribution function of the electrons is sometimes better approximated by one which is nowadays well-known as the Druyvesteyn distribution [86,87], i.e., $f_{E}(E) \propto \exp \left[-E^{2} /\left(k T_{\mathrm{e}}\right)\right]$. In comparison with Maxwellian distribution, Druyvesteyn distribution is characterized by depletion in the high-energy region and a shift of the maximum towards a higher energy ${ }^{2}$. Godayk et al. have argued that the use of standard techniques (as describe above) for analyzing probe data can lead to considerable errors in the resulting plasma parameters if the EEDF is non-Maxwellian [88]. These authors compared the results of applying various probe diagnostic techniques to non-Maxwellian plasmas [88]. Measurements were made in a $13.56 \mathrm{MHz}$ capacitive rf discharge in argon. At low pressures, typically $4 \mathrm{~Pa}$, the EEDF is observed to be bi-Maxwellian, indicating a cold electron swarm having a characteristic energy of $0.5 \mathrm{eV}$ and a density of $4.2 \cdot 10^{15} \mathrm{~m}^{-3}$ together with a hot group having a characteristic energy of $3.4 \mathrm{eV}$ and a lower density of $2.4 \cdot 10^{14} \mathrm{~m}^{-3}$. On the contrary, at higher pressures of $40 \mathrm{~Pa}$ the EEDF becomes the Druyvesteyn type, with a mean temperature of $3.4 \mathrm{eV}$ and a density of $2.9 \cdot 10^{15} \mathrm{~m}^{-3}$.

Further, the plasma parameters obtained from the energy distribution measurements were compared to those obtained by using conventional procedures assuming a Maxwellian distribution. The methods considered were the Druyvesteyn procedure involving the differentiation of the probe characteristic, and the classical procedure (as described above) applied to the electron retardation part of the probe characteristic. It was observed that the application of the classical methods to probe characteristics obtained in non-Maxwellian plasmas might lead to significant error by a factor of 2 to 5 . The degree of departure

2. To appreciate the qualitative difference between Maxwellian and Druyvesteyn distribution functions, the EEDFs of both types are available on: www.arjenboogaard.nl/druyvesteyn.html. 
of the EEDF from the Maxwellian in a particular discharge, and the resulting errors, are unpredictable. Based on this analysis, the Druyvesteyn procedure was used in this thesis, and will be introduced below.

The Druyvesteyn $[75,89,90]$ extension of the Langmuir and Mott-Smith probe theory [91-95] allows determination of the electron energy spectrum, regardless of its shape. Druyvesteyn showed that the EEDF can be obtained from expression:

$$
N(\varepsilon)=N_{\mathrm{e}} f_{\mathrm{E}}(E)=\frac{2}{e^{2} A} \sqrt{\frac{2 m_{\mathrm{e}} \varepsilon}{e}} \frac{\mathrm{d}^{2} I}{\mathrm{~d} V^{2}},
$$

where $N(\varepsilon)$ is the number of electrons within the energy domain $e(\varepsilon+\mathrm{d} \varepsilon)$ $\mathrm{eV}, V$ is the probe voltage, $\varepsilon$ is the probe voltage with respect to the plasma potential $V_{\mathrm{p}},\left(\varepsilon=V_{\mathrm{p}}-V\right), A$ is the probe surface area. The second derivative of the $I-V$ curve is derived numerically and averaged over 1000 measurements at each point on the $I-V$ curve to reduce noise $[75,96]$.

The electron density, $N_{\mathrm{e}}$, can be obtained from the integral of $N(\varepsilon)$,

$$
N_{\mathrm{e}}=\int_{0}^{\infty} N(\varepsilon) \mathrm{d} \varepsilon
$$

An evaluation of problems encountered in EEDF-measurements in inductively-coupled plasmas and some remedies to avoid them are reviewed in Godyak et al. [75], and in Scanlan and Hopkins [97].

\subsection{Optical Emission Spectroscopy (OES)}

Among a variety of optical diagnostic techniques for plasmas, the spectroscopy of emission induced by the plasma is the most widespread method due to its nonintrusiveness and easy implementation. For example, various types of OES measurements can yield electron temperatures, gas temperatures, plasma densities and species concentrations [98]. The underlying mechanism in most of these plasmas that leads to the excitation of the atoms and molecules is electronimpact excitation. Before describing the diagnostic technique that was used to measure plasma properties, a brief overview is provided of the underlying assumptions.

The ground state electron configuration of argon is $1 s^{2} 2 s^{2} 2 p^{6} 3 s^{2} 3 p^{6}$, which will be abbreviated as $3 \mathrm{p}^{6}$. Excited neutral levels are formed by exciting one of the $3 p$ electrons into an excited orbital (i.e., $n l$ ). Any given $3 p^{5} n l$ configuration gives rise to a number of energy levels. Since Ar does not conform 


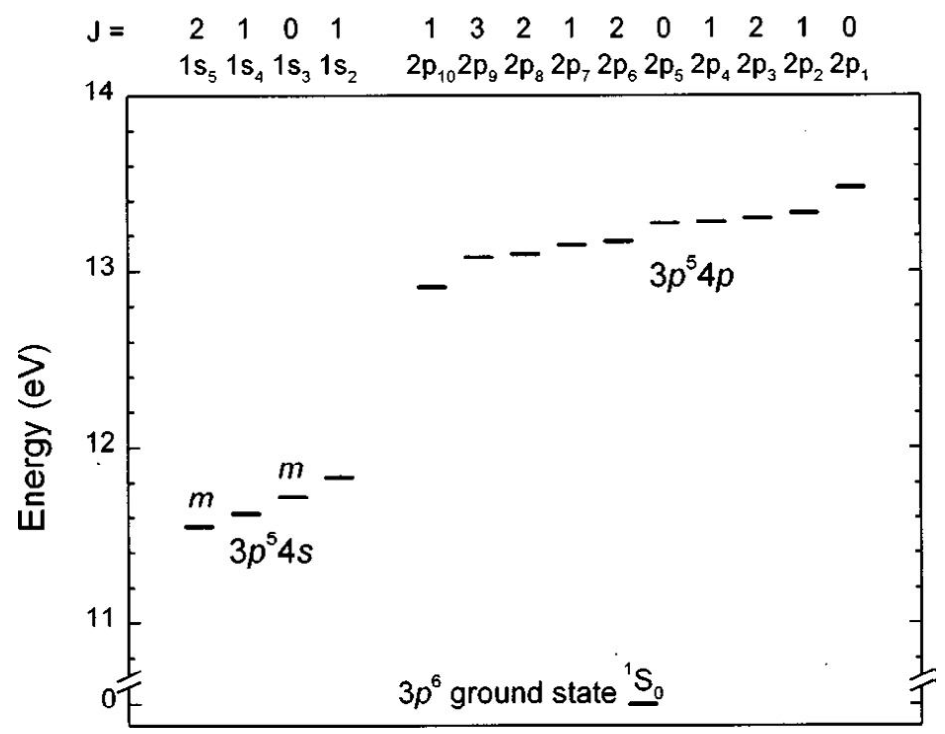

Figure 4.8: Simplified Argon energy level diagram; $m$ denotes metastable state.

to any of the standard coupling schemes (i.e., L-S, jj) [99], the excited levels of Ar are usually designated by either Paschen's notation [100], or by Racah's notation [101]. For simplicity, in this thesis we will generally designate atomic energy levels with Paschen's notation. Paschen's notation is strictly empirical: the lowest excited Ar energy $3 \mathrm{p}^{5} 4 \mathrm{~s}$ level is called the $1 \mathrm{~s}_{5}$, the second highest $1 s_{4}$, etc., see Figure 4.8. The next Ar configuration $3 p^{5} 4 p$ consists of ten levels. In Paschen's notation these are designated (from highest to lowest energy) the $2 \mathrm{p}_{1}$ to $2 \mathrm{p}_{10}$ levels.

\subsubsection{Corona versus saha equilibrium}

Bulk properties of a plasma can be described in terms of the interactions between atoms (and molecules), electrons, ions and photons. In a low-density plasma, the number density of atoms is low enough to safely neglect excitedstate atom-atom and excited-state ion-atom collisions. Furthermore, in a weakly ionized plasma the electron and ion densities are low in comparison with the atomic density. Thus, the primary channels for excitation/de-excitation of atoms are with electrons and photons. In corona equilibrium [101-103], applicable at low electron densities, atomic excitation is solely through electron impact 
excitation of ground state atoms and de-excitation is solely through photon decay. At the opposite extreme, in Saha-Boltzmann equilibrium, the electron density is so high that recombination or relaxation by electron collisions dominates radiative decays. In fact, by the principle of detailed balance the excitation and de-excitation rates are equal, so the excited state populations are independent of collision cross sections [104].

Although plasma conditions in this thesis are not typically low-density or weakly ionized, they do certainly not fulfill Saha equilibrium conditions. The figure of merit here is the radiative lifetime of the excited state compared to the mean time between electron-excited argon collisions. The latter can be calculated from equation (3.32), and measures $\tau \approx 0.3 \mathrm{~s}$ at typical process pressures conditions. Typical radiative lifetimes are $20-40 \mathrm{~ns}$ for $3 p^{5} 4 p \rightarrow 3 p^{5} 4 s$ emissions (700-1150 nm wavelength) [105-107], and 200-500 ns for $3 \mathrm{p}^{5} \mathrm{np} \rightarrow$ $3 \mathrm{p}^{5} 4 \mathrm{~s}(n=6,7)$ emissions (300-390 nm) [108]. Consequently, an excited atom is not likely to undergo an electron-atom collision before it decays radiatively. Hence, Saha conditions are not met, and the corona model will be used to describe plasma emissions.

\subsubsection{Line intensities from ground states in corona equilibrium}

Consider atoms in a small plasma volume excited by electron-atom collisions into an excited level $i$ (sketched in Figure 4.9). These atoms subsequently decay to one of the lower states $l$ (see Figure 4.10). In corona equilibrium, the electron excitation rate and photon decay rate are equal. The photon flux for a particular $i \rightarrow j$ transition, $\Phi_{i j}$, is equal to [109]

$$
\Phi_{i j}=\frac{A_{i j}}{\sum_{l<i} A_{i l}} k_{0 i} N_{0} N_{\mathrm{e}},
$$

where $N_{0}$ is the density of ground state atoms, $N_{\mathrm{e}}$ is the electron density, $k_{0 i}$ is the reaction rate constant of the ground state excitation into level $i$, and $A$ is the spontaneous emission rate. One can observe from the equation above that the photon flux from a particular emission depends upon both the excitation rate into the level, $k_{0 i}$, and the so-called branching fraction, $A_{i j} / \sum_{l<i} A_{i l}$, of the decays out of the level. Most excitation levels have more than one decay channel, see Figure 4.10. The likelihood of it decaying to a particular mode is known as its branching ratio or branching fraction for that decay mode. Generally speaking, prominent emission lines also have a more favorable branching fraction. A good example of this is the excitation into the $\operatorname{Ar}\left(2 \mathrm{p}^{9}\right)$ level (see Figure 4.8). This leads to a prominent emission line at $811.5 \mathrm{~nm}$, which is the only decay channel for this level [106]. 


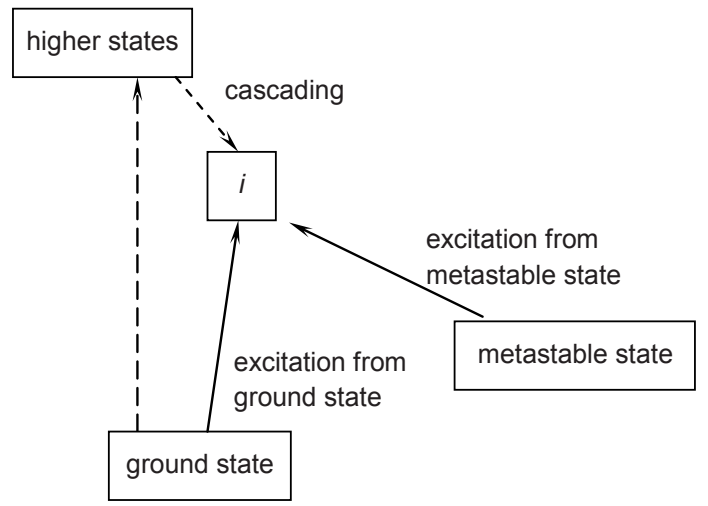

Figure 4.9: Schematic of processes that populate the levels.

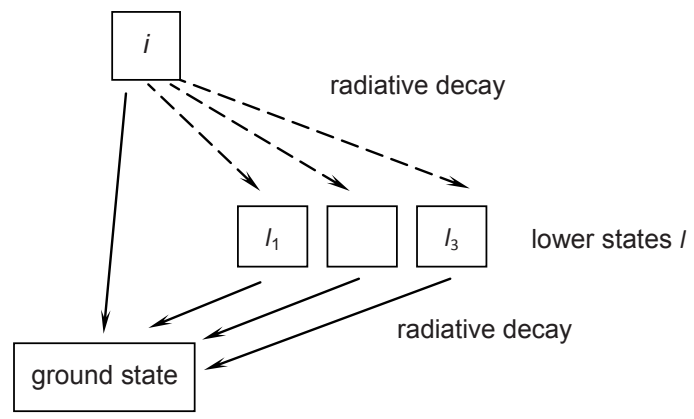

Figure 4.10: Schematic of processes that depopulate the levels. 
In section 3.2 it was explained that the rate constant can be found by the integration of the cross section over the velocity distribution function, $f_{v}(v)$. Equation (3.35) can be inserted into equation (4.20) to yield

$$
\Phi_{i j}=\frac{A_{i j}}{\sum_{l<i} A_{i l}} N_{0} N_{\mathrm{e}} \int \sigma_{0 i}(v) v f_{v}(v) \mathrm{d} v .
$$

Or expressed in terms of energy:

$$
\Phi_{i j}=\frac{A_{i j}}{\sum_{l<i} A_{i l}} N_{0} N_{\mathrm{e}} \sqrt{\frac{2}{m_{\mathrm{e}}}} \int \sigma_{0 i}(E) \sqrt{E} f_{\mathrm{E}}(E) \mathrm{d} E,
$$

where $m_{e}$ is the electron mass, $\sigma_{0 i}(E)$ is the ground state excitation cross section into level $i$ as a function of electron energy $E$, and $f_{\mathrm{E}}(E)$ is the electron energy distribution function.

It is important to note that different experimental and theoretical techniques yield different types of cross sections. Those differences can have large impact on calculations made by the equations above. One can speak of many different types of electron-atom excitation cross sections: optical, emission, apparent, direct, etc, a fact that can lead to some confusion. It is not wise to overlook the substantial difference between apparent cross sections measured in optical experiments [110] and direct cross sections measured by energy-loss electron spectroscopy [111]. To illustrate this, consider again excitation into the $\operatorname{Ar}\left(2 p_{9}\right)$ level (the $811.5 \mathrm{~nm}$ emission line). The apparent excitation cross section for this level has a peak cross section of $13 \cdot 10^{-18} \mathrm{~cm}^{2}$ at $18 \mathrm{eV}$, while the direct excitation cross section is only $5.6 \cdot 10^{-18} \mathrm{~cm}^{2}$ [106], i.e., less than half the apparent cross section. The difference between the two is explained by the effect that many levels receive a substantial fraction of their population from cascades of higher levels, see Figure 4.9. This cascading effect is included in the optical measurement of the apparent cross section, and not in the measurement of the direct cross section. Again taking the $\operatorname{Ar}\left(2 p_{9}\right)$ level as an example, the cascade contribution from the $4 \mathrm{~d}^{4}$ level, contributes $1.3 \cdot 10^{-18} \mathrm{~cm}^{2}$ to this total, while the contribution from the $3 \mathrm{~d}^{4}$ level at $18 \mathrm{eV}$ is even larger, $5.0 \cdot 10^{-18} \mathrm{~cm}^{2}$ [112]. Thus, the apparent cross section is the sum of the direct excitation cross section and the cascade cross section. Finally, the $i \rightarrow j$ optical emission cross section, $\sigma_{i j}^{\mathrm{Opt}}$, is related to the apparent cross section, $\sigma_{0 i}^{\mathrm{App}}$, but includes the branching ratio [108]:

$$
\sigma_{i j}^{\mathrm{Opt}}(E)=\frac{A_{i j}}{\sum_{l<i} A_{i l}} \sigma_{0 i}^{\mathrm{App}}(E) .
$$


Plasma diagnostic techniques are at an advantage using optical emission cross sections since individual transition intensities are the observed quantities. Using the optical emission cross section, equation (4.22) simplifies to

$$
\Phi_{i j}=N_{0} N_{\mathrm{e}} \sqrt{\frac{2}{m_{\mathrm{e}}}} \int \sigma_{i j}^{\mathrm{Opt}}(E) \sqrt{E} f_{\mathrm{E}}(E) \mathrm{d} E .
$$

Note that $\Phi_{i j}$ is the photon flux radiated into all directions from a small volume element of the plasma. The observed photon flux (photons $\cdot \mathrm{s}^{-1}$ ) is the integral of this photon flux over the line of sight of the optical detection system weighted by the solid angle of the optical system (photons $\cdot \mathrm{s}^{-1} \cdot \mathrm{sr}^{-1}$ ), typically absorbed into the catchall constant $C$. An intensity value $\left(\mathrm{W} \cdot \mathrm{sr}^{-1}\right)$ is obtained if we multiply the photon flux by the energy per photon: $h v_{i j}$, where $h$ is Planck's constant, and $v_{i j}$ is the frequency of transition $i \rightarrow j$.

So far, we have made no assumptions regarding the functional form of the EEDF, $f_{\mathrm{E}}(E)$. In practice, one of several standard functional (Maxwellian, biMaxwellian or Druyvesteyn) forms is typically assumed. Combining the simplifications of a Maxwellian distribution ( $c f$. equation (3.4)) with constant $C$, the line intensity, $I_{i j}$, incident on the detector for a particular $i \rightarrow j$ emission line is equal to

$$
I_{i j}=C h v_{i j} N_{0} N_{\mathrm{e}}\left(k T_{\mathrm{e}}\right)^{-3 / 2} \int \sigma_{i j}^{\mathrm{Opt}}(E) \exp \left(\frac{-E}{k T_{\mathrm{e}}}\right) E \mathrm{~d} E .
$$

\subsubsection{Excitation from non-ground states}

The corona model, and therefore equation (4.24), should be handled with care however, since another basic assumption, "atomic excitation is solely through electron impact excitation of ground state atoms", is not always met, see Figure 4.9. Some levels populate from nearby states as well. The $3 p^{5} 4 p \rightarrow 3 p^{5} 4$ s emissions ( $2 \mathrm{p} \rightarrow 1 \mathrm{~s}$ in Paschen's notation) for example, which lie in the 700-1150 $\mathrm{nm}$ wavelength range, are the most intense lines of the argon spectra in the visible/near-IR. The population dynamics of these levels in plasmas may be described by equation (4.24) since the excitation cross sections into the $3 \mathrm{p}^{5} 4 \mathrm{p}$ levels are relatively large $\left(\sim 10^{-17}-10^{-18} \mathrm{~cm}^{2}\right)$ [105-107]. In contrast, levels close to the ionization energy have smaller excitation cross sections from the ground state, but large electron-impact excitation cross sections to and from nearby excited states, due to the small energy differences between these levels. Typical examples are many of the $3 p^{5} n p \rightarrow 3 p^{5} 4 s(n=6,7)$ emission lines (300-390 nm) (4p $\rightarrow 1$ s and 5p $\rightarrow 1$ s in Paschen's notation) [108].

Also the $3 p^{5} 4 p \rightarrow 3 p^{5} 4$ s emissions as described above have to be handled with care. The $J=0\left(1 \mathrm{~s}_{3}\right.$ in Paschen's notation) and $J=2\left(1 \mathrm{~s}_{5}\right)$ levels of the 
$3 \mathrm{p}^{5} 4 \mathrm{~s}$ configuration are metastable with lifetimes over $1 \mathrm{~s}$. Cross sections have been measured for excitation out of these levels into the $3 p^{5} 4 p$ levels $[113,114]$. These cross sections are generally much larger $\left(10^{-19}-10^{-20} \mathrm{~m}^{2}\right)$ than the corresponding cross sections out of the ground state $\left(\sim 10^{-21}-10^{-22} \mathrm{~m}^{2}\right)$. Excitation of metastable states would lead to increased emissions over those predicted from electron-impact excitation of the ground state; in fact, it can lead to a significant fraction of the emissions for some levels in a discharge $[115,116]$. Cross section measurements and plasma modeling and experiments however point to a minuscule role for metastable excitation of the $n p_{1}, n p_{5}(J=0)$ levels. The emissions from these levels can usually be assumed completely free of a metastable atom excitation contribution. In fact, the Ar emission lines from these safe levels $(750.4,751.5,425.9,451.1 \mathrm{~nm})$ are the ones most often used in line-ratio measurements [117]. Alternatively, the modeled line intensity can include a contribution from both metastable and ground state excitation (replacing $N_{0}$ and $\sigma_{i j}^{\mathrm{Opt}}$ by the appropriate values) since the metastable excitation cross sections are known [118]. An advantage of this approach is that the onset energy from metastable levels is much lower $(\sim 2 \mathrm{eV})$ than the ground state onset $(>11.56 \mathrm{eV})$; thus, the analysis stretches out over a much wider range of the EEDF.

Two-step excitation has to be accounted for when studying ion line intensities as well. An excited $\mathrm{Ar}^{+}$level can be reached by either the one-step process,

$$
\mathrm{Ar}+\mathrm{e}^{-}(E>35 \mathrm{eV}) \rightarrow \mathrm{Ar}^{+*},
$$

or the two-step process,

$$
\begin{aligned}
& \mathrm{Ar}+\mathrm{e}^{-}(E>15.5 \mathrm{eV}) \rightarrow \mathrm{Ar}^{+} \\
& \mathrm{Ar}^{+}+\mathrm{e}^{-}(E>19.5 \mathrm{eV}) \rightarrow \mathrm{Ar}^{+*}
\end{aligned}
$$

For a typical plasma ionization fraction, $\alpha_{\mathrm{iz}}=3 \cdot 10^{-3}$, the cross section results of Zapesochnyi et al. [119] would predict that the two-step contribution from excitation of ions will contribute more than half of the total photon flux on the $488.0 \mathrm{~nm} \mathrm{Ar}^{+*}$ emission line for all electron temperatures below $7 \mathrm{eV}$. Fuller et al. [120] even found that under their conditions (i.e., a $\mathrm{Cl}_{2}-\mathrm{Ar}$ ICP plasma) the contribution of one-step excitation of Ar to produce $\mathrm{Ar}^{+*}$ was unimportant. While the possibility of two-step excitation of ions is sometimes mentioned in the analysis of line-ratio measurements, there have been few attempts to correct for this effect [121,122]. Nonetheless, there are two relatively easy ways to address this problem within the confines of the corona model. First, the use 
of ion lines can be avoided. Second, if ion lines are used, the modeled signal for the ion line should include the two-step contribution [120,123],

$$
I_{i j}^{\text {Obs }}=I_{i j}^{\text {one step }}+I_{i j}^{\text {two step }},
$$

where $I_{i j}^{\text {Obs }}$ is the measured line intensity, $I_{i j}^{\text {one step }}$ and $I_{i j}^{\text {two step }}$ are the calculated intensities using the one-step and two-step excitation mechanisms, respectively.

\subsubsection{Line ratios}

A single plasma intensity measurement cannot uniquely determine the electron temperature since the photon flux in equation (4.24) depends upon both the electron density and EEDF. To overcome these difficulties, it is common to measure the line ratio of two emission lines, which is only dependent on the EEDF.

There have been several attempts to use OES to measure $k T_{\mathrm{e}}$ or EEDF. The general approach is to compare relative intensities of emission lines that have different excitation energies [39, 47-49,124-129]. Typically, two emission lines are compared, and the EEDF is assumed to be Maxwellian:

$$
\frac{I_{i j}}{I_{a b}}=\frac{h v_{i j} \int_{E_{1}}^{\infty} \sigma_{i j}^{\mathrm{Opt}}(E) \exp \left(-E / k T_{\mathrm{e}}\right) E \mathrm{~d} E}{h v_{a b} \int_{E_{2}}^{\infty} \sigma_{\mathrm{ab}}^{\mathrm{Opt}}(E) \exp \left(-E / k T_{\mathrm{e}}\right) E \mathrm{~d} E},
$$

where $I_{i j}$ and $I_{a b}$ are the intensities of emission lines assigned to transitions $i \rightarrow j$, and $a \rightarrow b$, while $v_{i j}, v_{a b}$, and $E_{1}$ and $E_{2}$ are their corresponding frequencies and onset energies, respectively. The sensitivity of this method strongly depends on the difference in excitation energies of the chosen transition lines, or their cross sections must be radically different.

There are several publications on a method to measure electron temperatures by the so-called trace rare gases optical emission spectroscopy (TRG-OES) (see Donnelly [130] and references therein). In this method, small amounts of each of five noble gases are added to the plasma (about 1\% each, so $5 \%$ in total), and $k T_{\mathrm{e}}$ is determined from the relative intensities of about 20 emission lines. In this work, we used the two-emission-line approach, both for its relative simplicity, and to avoid the addition of other gases than our precursors to the plasma.

\subsubsection{Equipment}

The spectra were acquired using an optical fiber positioned at the quartz window mounted on the chamber wall (item (11) in Figure 4.1). A setup with a 
small acceptance angle (a few degrees) was chosen such that only light propagated that entered the fiber within a small acceptance cone. This enabled us to perform measurements localized to the Langmuir Probe. The fiber output was connected to an Avantes AVS-SD2000 spectrophotometer with an optical resolution of $0.5 \mathrm{~nm}$. The measured spectral range was $250-1100 \mathrm{~nm}$. A calibrated halogen lamp HL-2000 has been used as reference for absolute measurements.

Figure 4.11 shows typical emission spectra obtained from pure Ar, Ar$\mathrm{N}_{2} \mathrm{O}$, and $\mathrm{Ar}-\mathrm{SiH}_{4}$ plasmas. There are several observations that can be made from these spectra. First, in the spectral range of 560 to $700 \mathrm{~nm}$, we observe Lewis-Rayleigh afterglow of nitrogen. This is typically observed for $\mathrm{Ar}-\mathrm{N}_{2} \mathrm{O}$ [131] and $\mathrm{Ar}-\mathrm{N}_{2}$ plasmas [132-135]. Second, the addition of $\mathrm{SiH}_{4}$ to Ar plasma results in an extra lines at $656 \mathrm{~nm}$, which can be ascribed to Balmer- $\alpha$ emission from atomic hydrogen $(\mathrm{H} \alpha)[134,135]$. Other emission lines due to $\mathrm{SiH}_{4}$ dissociation are found at $486 \mathrm{~nm}(\mathrm{H} \beta), 414 \mathrm{~nm}\left(\mathrm{SiH}^{*}\right)$, and at $255 \mathrm{~nm}$ and $288 \mathrm{~nm}$ (both $\mathrm{Si}^{*}$ ) [135]. $\mathrm{Si}^{*}$ (emitting at 288 and $255 \mathrm{~nm}$ ) and $\mathrm{SiH}^{*}$ (emitting at $414 \mathrm{~nm}$ ) are excited states that are formed by dissociation of $\mathrm{SiH}_{4}$ (i.e., one-step process), whereas the Balmer alpha $\mathrm{H} \alpha(656 \mathrm{~nm})$ and Balmer beta line $\mathrm{H} \beta(486 \mathrm{~nm})$ are associated with atomic hydrogen. From the recorded spectra, the intensity of the chosen lines is quantified after subtraction of the background signal. 


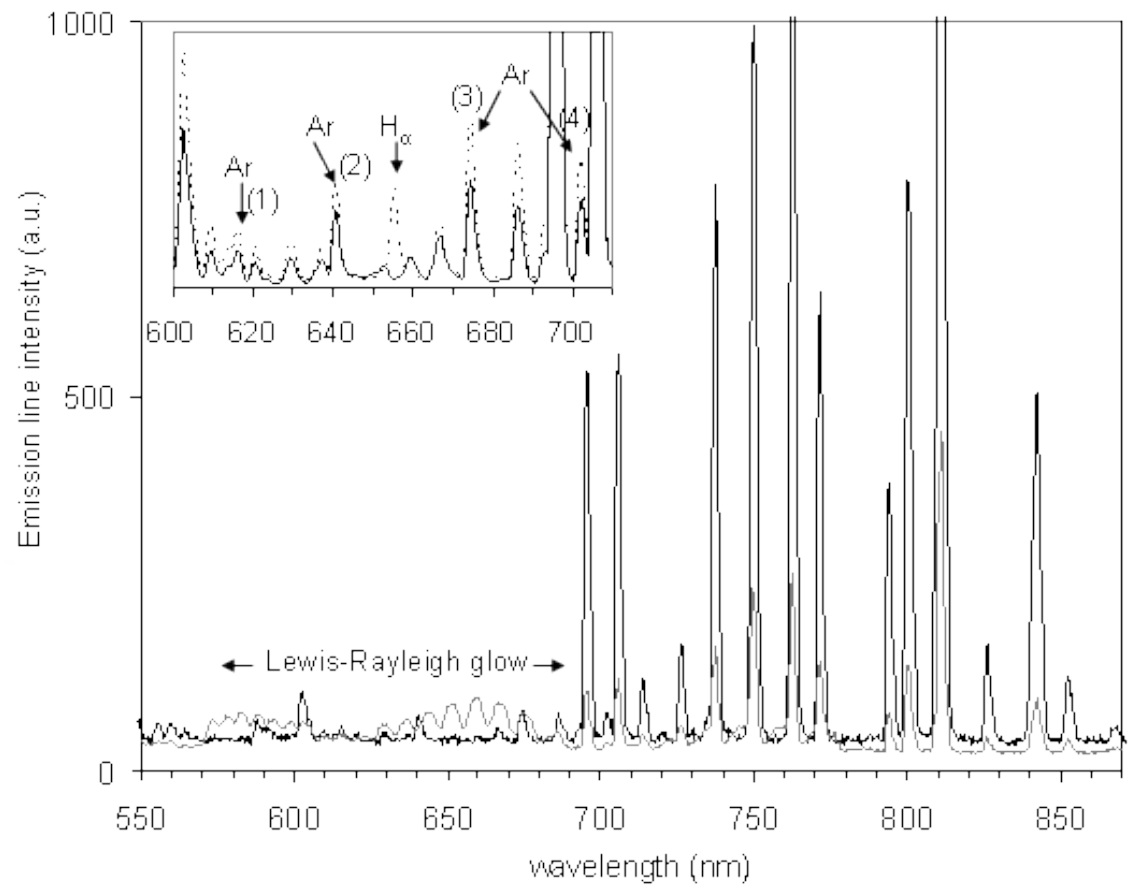

Figure 4.11: Typical $\mathrm{Ar}, \mathrm{Ar}-\mathrm{N}_{2} \mathrm{O}$, and $\mathrm{Ar}-\mathrm{SiH}_{4}$ plasma emission spectra recorded at a position of $240 \mathrm{~mm}$ on the vertical axis shown in Figure $4.1 ; p=1 \mathrm{~Pa}$, ICP power $300 \mathrm{~W}$. The insert shows spectra of pure $\mathrm{Ar}$ and $\mathrm{Ar}-\mathrm{SiH}_{4}$ plasmas within a smaller wavelength-range. (-) Ar plasma (Ar flow $200 \mathrm{sccm}$ ); (-) Ar- $\mathrm{N}_{2} \mathrm{O}$ plasma (Ar flow: $200 \mathrm{sccm}, \mathrm{N}_{2} \mathrm{O}$ flow: $44 \mathrm{sccm}$ ); (- - ) $\mathrm{Ar}_{-} \mathrm{SiH}_{4}$ plasma (100 sccm of $\mathrm{Ar}+100 \mathrm{sccm}$ $\mathrm{SiH}_{4}-$ Ar flow ( $2 \% \mathrm{SiH}_{4}$ in $\mathrm{Ar}$ ). 


\section{Experimental characterization of the discharge}

\subsection{Introduction}

Knowing electron densities $\left(N_{\mathrm{e}}\right)$ and electron energy distribution functions (EEDF) is of prime importance for the modeling and optimization of chemical and physical processes in plasma reactors [9-15]. Assuming, for example, a Maxwell-Boltzmann energy distribution for electrons in high-density plasma reactors operating at low gas pressures (e.g., ECR and ICP reactors) can result in significant modeling and interpretation errors because a fraction of electrons might deviate from thermal equilibrium [88]. One normally uses a Langmuirprobe to obtain EEDFs, but these measurements are hardly possible in depositing plasmas due to the formation of deposits on the probe (see Perry et al. [136] and references therein). One can also use optical emission spectroscopy (OES) to obtain characteristic electron temperatures $\left(k T_{\mathrm{e}}\right)$ in plasmas from line ratio measurements, see section 4.3. To do so, one should first determine the functional form of the EEDF by a Langmuir probe measurement. Equation (4.27) can be used to obtain $k T_{\mathrm{e}}$ from the measured line rations if the EEDF appears to be a single Maxwellian. A similar equation can be derived for Druyvesteyn functions. However, an EEDF is not well described by a $k T_{\mathrm{e}}$ value when it deviates from a Maxwellian or Druyvesteyn description. When this is the case, the line ratio method can still be used to verify the EEDF measurements performed by a Langmuir probe. The approach is to numerically calculate the line intensities from the experimentally obtained EEDFs and optical cross sections. The measured and calculated ratios of two emission lines are then compared. The measured EEDF is assumed to be accurately described by de LP measurement 
if the two ratios coincide.

A method was developed to obtain full-range EEDFs for plasmas with silane precursor for thin film deposition of silicon oxide (from $\mathrm{Ar}-\mathrm{N}_{2} \mathrm{O}-\mathrm{SiH}_{4}$ mixtures). Our method comprises three steps. First, EEDFs of (non-depositing) Ar-plasmas were measured by a Langmuir-probe, while line ratios were obtained by OES under the same conditions. Second, we verified that, in cases of small additions of other (still non-depositing) gases, Langmuir-probe and OES methods would give the same results. Therefore, non-depositing precursors $\left(\mathrm{N}_{2}\right.$ and $\left.\mathrm{N}_{2} \mathrm{O}\right)$ were mixed into the Ar-plasma. Third, small amounts of silane were added to $\mathrm{Ar}, \mathrm{Ar}-\mathrm{N}_{2}$, and $\mathrm{Ar}-\mathrm{N}_{2} \mathrm{O}$ plasmas, and the change in line ratios was monitored by OES. At a certain "threshold" concentration of silane, line ratios started to deviate from that of plasmas without silane. Below that concentration, we assume that EEDFs measured in the plasmas without silane can be used.

A number of researchers have compared $k T_{\mathrm{e}}$ values obtained with both techniques for various plasmas $[39,48,49,125-128]$. Our method adds to this literature in (i) its uniqueness to obtain full-range EEDFs, regardless their shape, and (ii) its applicability in depositing plasmas.

The method allows for modeling of depositing plasmas where experimental data of non-depositing are used as input parameters, and can thus minimize modeling errors. The latter is essential for a better understanding and optimization of the deposition process.

A large part of this work was published in $[137,138]$.

\subsection{Argon plasmas}

A typical example of an EEDF in Ar plasma is shown in Figure 5.1. It is important to note that zero energy at the $x$-axis means that the probe is at plasma potential. Similarly, $50 \mathrm{eV}$ corresponds to a negative probe potential of $-50 \mathrm{~V}$ with respect to plasma potential. The energies are therefore always measured with respect to plasma potential. The vertical axis depicts the number of electrons within the energy domain $e(\varepsilon+\mathrm{d} \varepsilon)$. The straight lines correspond to the Maxwell-Boltzmann approximation of the energy distribution for a system in thermal equilibrium. One can see that the largest fraction of the electrons in this plasma is indeed in thermal equilibrium. However, we also measure a fraction of electrons ( $\sim 0.5 \%$ of their total concentration) in the energy range $20-50 \mathrm{eV}$ that deviate from thermal equilibrium.

It is to be expected from theoretical considerations that a part of the energy distribution is non-Maxwellian. The Maxwell-Boltzmann distribution was 
derived for a system of particles in an equilibrium situation free from the action of external forces [139]. A (rf) plasma is not in complete equilibrium since electrons can gain energy between collisions under the influence of the electromagnetic field. There are three main theories that can describe the appearance of fast electrons in rf discharges. The first theory involves elastic collisions of electrons with argon atoms at an appropriate time with respect to the phase of the electric field. If an electron makes an elastic collision, reversing its motion at the exact moment the field changes direction, then its velocity and energy will continue to increase [140-142]. A second group of theories claims that the secondary electrons, which are emitted from the walls and are accelerated across the positive ion sheath into the plasma, act as an additional supply of electrons. If the electric field changes its direction at the required time, this can lead to an efficient ionization [143]. Emission of electrons from surfaces can be induced by photons (photo-emission), the electric field (field-induced emission), or by incoming ions $[45,46,144]$. The third theory attributes energy gain of electrons to surf riding (or wave riding) of electrons on the oscillating edge of the plasma sheath $[45,145,146]$.

None of these theories however was able to explain the appearance of the high-energy electron tail. At this time, it is not clear which process causes the appearance of fast electrons in our system. A detailed analysis of these mechanisms is required, including electromagnetic modeling of our plasma system, for further understanding. However, some general remarks concerning modeling can already be made.

Although electrons with energy of $15.76 \mathrm{eV}$ can ionize argon atoms to $\mathrm{Ar}^{+}$[32], the ionization cross section is still very small $\left(\sim 1 \cdot 10^{-22} \mathrm{~m}^{2}\right)$. The ionization cross section increases rapidly for electrons with energies higher than the onset energy, until a maximum $\left(\sim 2 \cdot 10^{-20} \mathrm{~m}^{2}\right)$ is reached at energies between 40 and $200 \mathrm{eV}$ [32]. Furthermore, an electron in a gas may take part in several processes, such as elastic scattering, excitation, ionization, and attachment. There is a certain probability for each of these processes to take place, expressed as an individual collision cross section. The total cross section is just the sum of the individual cross sections. For electrons in argon plasma, the total collision cross section peaks at about $14 \mathrm{eV}$ [147]. This can play a role since it appears to be the value at which our EEDF starts to deviate from the Maxwell-Boltzmann equation (see Figure 5.1).

These fast electrons can significantly influence the plasma composition, which makes them important for the chemical modeling. This is illustrated in Table 5.1. It shows the reaction rate constants of excitation and ionization in argon plasma for both the Maxwell-Boltzmann and experimentally mea- 


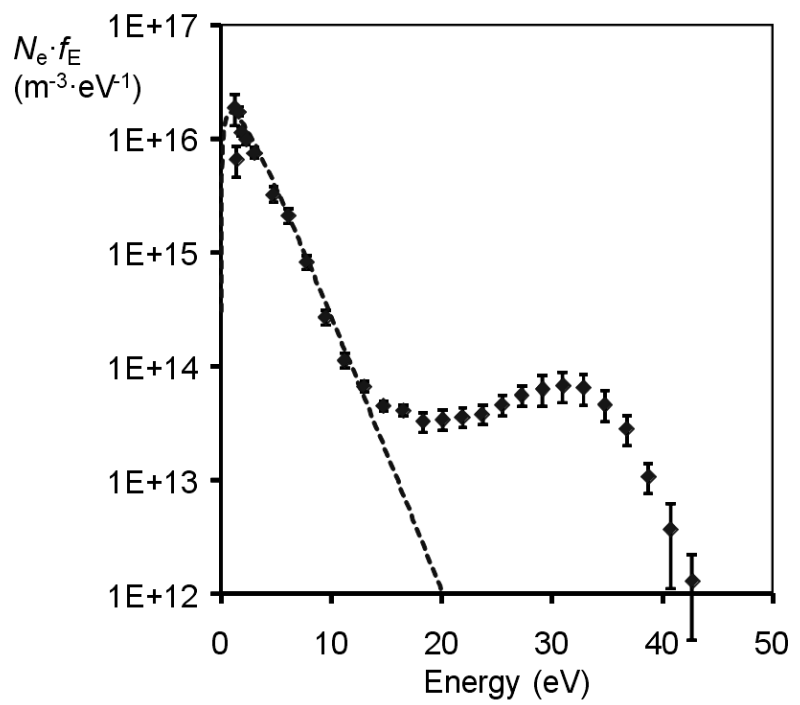

Figure 5.1: EEDF for Ar plasma at $1 \mathrm{~Pa}$. Probe position $80 \mathrm{~mm}$; ICP power $300 \mathrm{~W}$. The symbols represent the measurements and the dotted line represent the MaxwellBoltzmann distribution fitted to the measurement (fitting parameters: $N_{\mathrm{e}}=5 \cdot 10^{16}$ $\mathrm{m}^{-3}$ and $\left.k T_{\mathrm{e}}=1.7 \mathrm{eV}\right)$.

sured energy distribution at $1 \mathrm{~Pa}$. The appropriate $k_{\mathrm{exc}}$ and $k_{\mathrm{iz}}$ were calculated from equation (3.35) by numerical integration of the corresponding excitation and ionization cross sections [32] and the experimental or Maxwellian EEDF from Figure 5.1. The reaction rate constant for excitation is in our case 20 times higher than Maxwell-Boltzmann distribution predicts. For ionization, which has higher onset energy, the difference between the Maxwell-Boltzmann distribution and experiment becomes orders of magnitude. To successfully model chemical reactions in plasma, we certainly have to measure the actual EEDF, especially since it turns out to be non-Maxwellian.

Figure 5.2 shows the electron energy distribution at several pressures $(1,6$ and $12 \mathrm{~Pa})$ and at several probe locations in the system (i.e., $80 \mathrm{~mm}, 180$ $\mathrm{mm}$ and $240 \mathrm{~mm}$ ). Comparing the energy distributions of the slow electrons at different pressures, one can see that the mean electron energy decreases with increasing pressure. This can be explained by the shorter mean free path at higher pressures that reduces the acceleration time between two collisions. Furthermore, the peak corresponding to fast electrons shifts to lower energies 
Table 5.1: Rate constants of excitation and direct ionization in an Ar-plasma with their onset energies $\left(E_{0}\right)$ [32]. Rate constants are calculated for the energy distribution measured at $1 \mathrm{~Pa}$ and for the Maxwell-Boltzmann distribution (cf. equation (3.4)) with a characteristic temperature of $1.7 \mathrm{eV}$ (see Figure 5.1).

\begin{tabular}{lrrr}
\hline \multirow{2}{*}{ Reaction } & & \multicolumn{2}{c}{ rate constant $\left(\mathrm{m}^{3} \mathrm{~s}^{-1}\right)$} \\
\cline { 3 - 4 } $\mathrm{e}+\mathrm{Ar} \rightarrow \mathrm{Ar}^{*}+\mathrm{e}$ & 11.56 & $2.1 \cdot 10^{-16}$ & $1.8 \cdot 10^{-17}$ \\
$\mathrm{e}+\mathrm{Ar} \rightarrow \mathrm{Ar}^{+}+2 \mathrm{e}$ & 15.76 & $2.2 \cdot 10^{-16}$ & $3.1 \cdot 10^{-18}$ \\
\hline
\end{tabular}

at higher pressures. This could also be caused by the decreased mean free path by which the fast electrons can gain less energy from the electric field.

With respect to the EEDF at $1 \mathrm{~Pa}$ (see Figure 5.2; $1 \mathrm{~Pa}$ ), it appears that the tail of fast electrons shifts to higher energies with an increasing distance from the ICP-source: compare the rhombic symbols (measured at $80 \mathrm{~mm}$, which is also the position of the ICP coil) with the triangles (measured on $180 \mathrm{~mm}$ ) and the circular symbols $(240 \mathrm{~mm})$. This observed effect is quite opposite of what is desired for a remote plasma system, namely a minimization of the substrate bombardment with fast electrons. At higher pressures this shift to higher energies decreases (Figure 5.2; $6 \mathrm{~Pa}$ ), or disappears (Figure 5.2; $12 \mathrm{~Pa}$ ).

The observed tail's shift (at $1 \mathrm{~Pa}$ ) towards higher energies with respect to the distance from the ICP-source will also affect the difference between the plasma and floating potentials, $V_{\mathrm{p}}-V_{\mathrm{f}}$. This is shown in Figure 5.3 for an argon plasma at $1 \mathrm{~Pa}$, where these two potentials are plotted as a function of the position of the probe. One can observe that the floating potential shifts to more negative values, while the plasma potential increases in relation with the distance from the ICP-source (at least to a position of $280 \mathrm{~mm})$. Hence, $\left(V_{\mathrm{p}}-V_{\mathrm{f}}\right)$ increases in relation with the distance from the ICP source. This is in line with theory since a higher average electron velocity will increase the electron flux to the probe sheath (cf. equation (3.5)) and a higher electric field is needed to balance the electron and ion fluxes (cf. equation (3.11)).

It was introduced in section 4.1 that the ion bombardment onto the chuck could be increased by applying an rf bias. Applying an rf bias results in a decrease of the floating potential with respect to the plasma potential (although it is then called "dc bias" or "dc shift") and hence an increase of $\left(V_{\mathrm{p}}-V_{\mathrm{f}}\right)$ [148]. From Figure 5.3 we can observe that choosing a proper position of the chuck is yet another way to control the ion bombardment. 


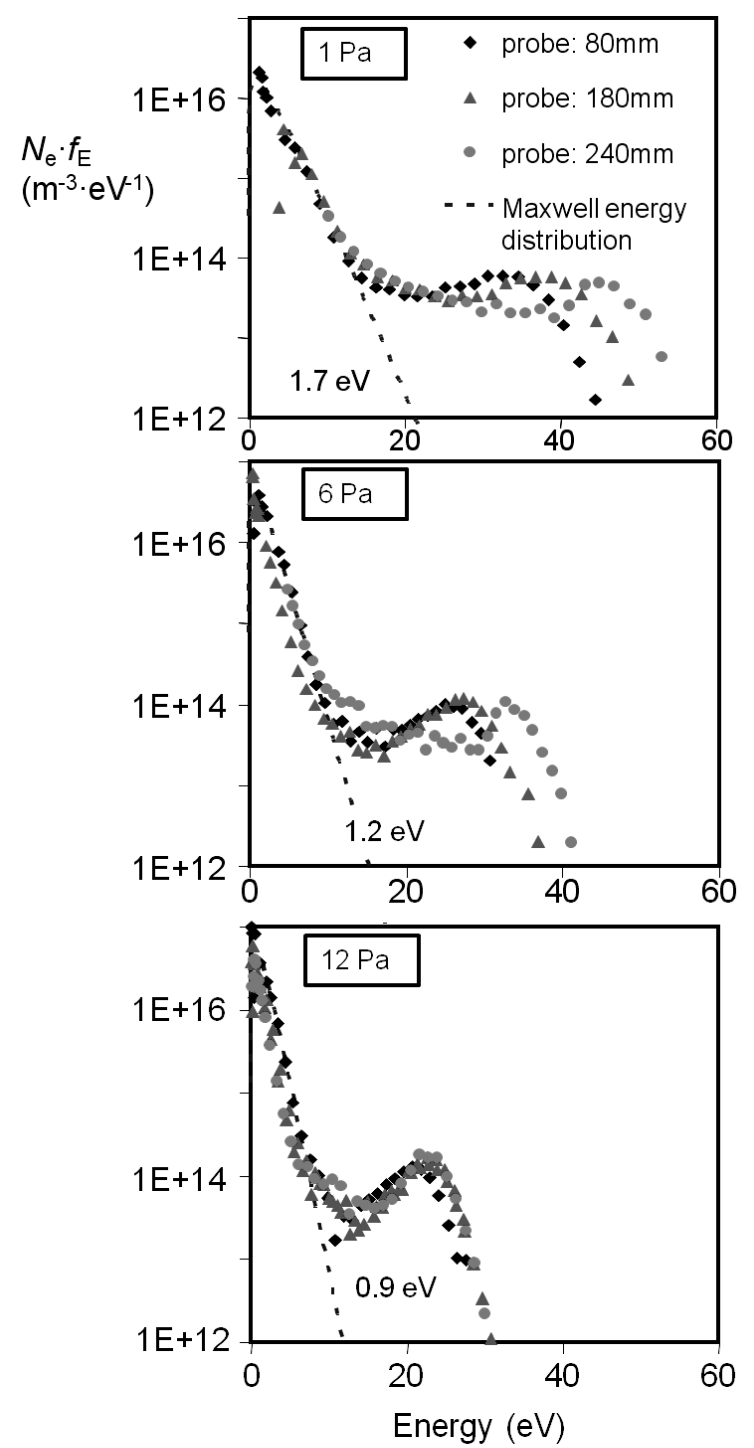

Figure 5.2: EEDFs for Ar plasmas at several pressures and probe positions. ICP power: $300 \mathrm{~W}$. The symbols represent the measurements and the dotted lines represent the Maxwell-Boltzmann distribution fitted to those measurements. Characteristic electron energies used for the Maxwell-Boltzmann distributions are listed in the figures. 


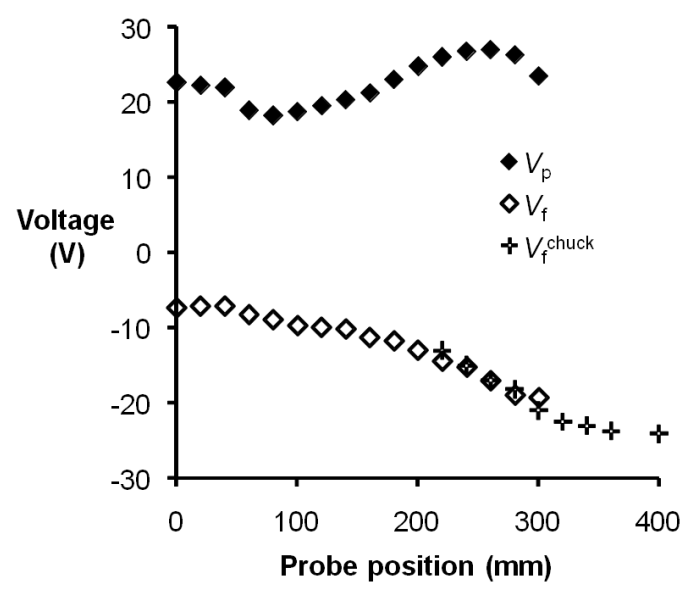

Figure 5.3: Plasma and floating potentials at several probe positions. ICP power: 300 $\mathrm{W} ; 1 \mathrm{~Pa}$ total argon pressure. The rhombic symbols represent the potentials deduced from the Langmuir probe measurements, whereas the plus signs represent the chuckbias measurements.

\subsubsection{First verification of LP measurements}

One can use the rf bias equipment to check whether or not the Langmuir probe measures proper floating potentials. This potential arises on every electrically floating object that is in contact with the plasma. So, a "dc bias" measured on the chuck without an rf bias applied (via a large capacitance), is actually the same floating potential as derived from the Langmuir probe measurements. The plus signs in Figure 5.3 represent the measured $V_{\mathrm{f}}^{\text {chuck }}$ as a function of the position of the chuck in an argon plasma. These measurements coincide with the Langmuir-probe measurements and hence the Langmuir probe measurements pass their first verification test.

\subsubsection{The variable axial magnetic field}

The ionization degree of the plasma can be increased by applying an external axial magnetic field. Due to the Lorentz force, the length of the electron trajectory is increased, which results in more collisions and better ionization efficiency, as was explained in section 4.1.1. The influence of a magnetic field is shown in Figure 5.4. In these experiments we measured the electron density at several positions in the system ranging from $0 \mathrm{~mm}$ to $300 \mathrm{~mm}$ along the axis shown in Figure 4.1. The highest electron density can be seen at the rf coil 


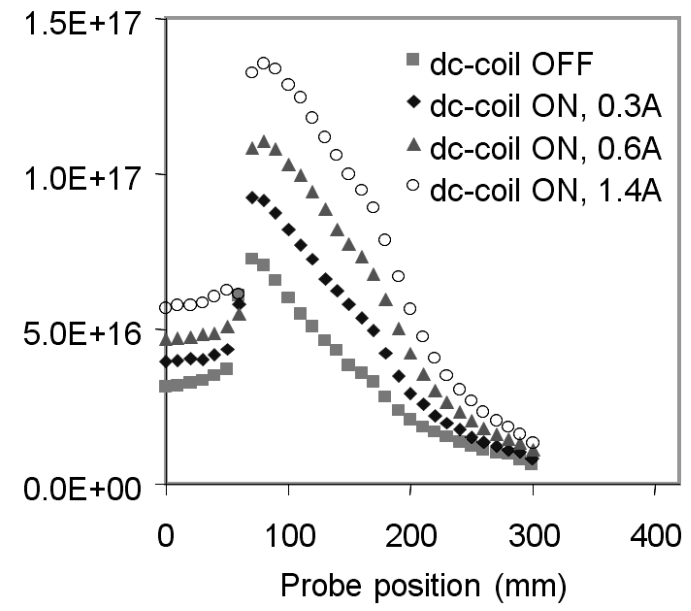

Figure 5.4: Electron densities in Ar plasma (1 Pa, $300 \mathrm{~W}$ ICP power) versus probe locations in the system, plotted for several magnetic field strengths. ( $\mathbf{\square}) B=0 \mathrm{~T}$; ( $)$ $B=2 \mu \mathrm{T} ;(\boldsymbol{\Delta}) B=4 \mu \mathrm{T} ;(\circ) B=9.5 \mu \mathrm{T}$. rf-coil is positioned at $80 \mathrm{~mm}$.

position (i.e., $80 \mathrm{~mm}$ ), with a maximum electron density of $N_{\mathrm{e}}=7 \cdot 10^{16} \mathrm{~m}^{-3}$ without an external magnetic field. The density of argon atoms at $1 \mathrm{~Pa}$ can be calculated by Avogadro's law to be $N_{\mathrm{g}}=2.4 \cdot 10^{20} \mathrm{~m}^{-3}$ [34]; assuming that the ion density $\left(N_{\mathrm{i}}\right)$ roughly equals the electron density, the maximum ionization degree, $\alpha_{\mathrm{iz}}$, of this plasma is $2.9 \cdot 10^{-4}$ at these conditions (see equation (3.1)). Then, a gradual decrease in electron density can be seen with increasing distance from the rf coil towards the chuck. This decrease is caused by capture of electrons on the reactor walls. Electron density increases as the intensity of the axial magnetic field is increased, which is in line with theory. Measurements show that the electron density increases from $N_{\mathrm{e}}=7 \cdot 10^{16} \mathrm{~m}^{-3}$ without magnetic field to $N_{\mathrm{e}}=1.4 \cdot 10^{17} \mathrm{~m}^{-3}$ with an external magnetic flux density of $9.5 \mu \mathrm{T}$.

The EEDF is also affected by the external magnetic field; this is shown Figure 5.5. The EEDFs in Figure 5.5a were measured without an external magnetic field at three positions in the chamber. Figure 5.5b and Figure 5.5c show the energy distributions at the same positions but in the presence of a magnetic field. By increasing the field, the distributions shift to lower energies. This can be explained as follows. Electron trajectories are deviated by a Lorentz force, which is proportional to the product of magnetic field intensity and electron 


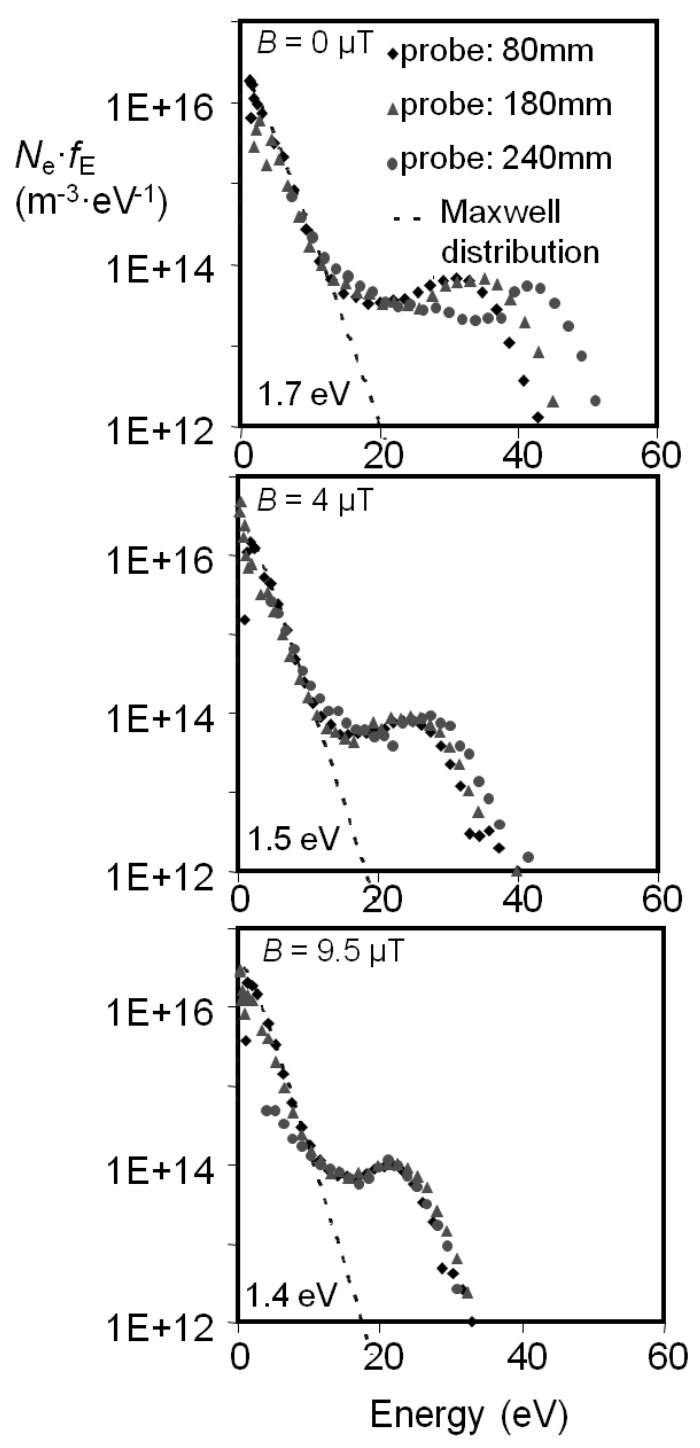

Figure 5.5: EEDFs for Ar plasmas (1 Pa, $300 \mathrm{~W}$ ICP power) for several magnetic flux densities and probe positions. The symbols correspond to measurements and the dotted line represents the Maxwell-Boltzmann distribution fitted to those measurements. Characteristic electron energies used for Maxwell-Boltzmann distributions are listed in the figures. 
velocity. So, fast electrons are influenced more effectively in such plasma than slow electrons. This will result in more collisions for fast electrons.

\subsubsection{Second verification of the LP measurements}

It was illustrated in Table 5.1 that fast electrons significantly influence the reaction rate constants of excitation and ionization. Based on these calculations, it was argued that these fast electrons are very important for the plasma composition and they should not be ignored. One can also state however, that their existence should be verified before we conclude on them, especially since the shape of the measured fast-electron tails could not be described by a known theory.

The reader should recall that these fast electrons also influence the emission spectrum of a plasma since the photon flux from a particular emission depends upon the excitation rate (cf. equation (4.20)). That is why OES can be used to verify the EEDF measurements as performed by the Langmuir probe. The approach was to calculate line intensities from the experimentally obtained EEDFs and the corresponding optical cross sections. Afterwards, ratios of two emission lines were calculated, and compared with the measured line ratios. The chosen transitions are tabulated in Table 5.2. This table also shows the excitation processes used to calculate the line intensities. The reader is referred to section 4.3 for an analysis on these processes. An example on how to calculate these ratios is given in Appendix A. The emission lines were chosen such that there was no overlap with lines from other plasma species and their optical cross sections were available. Figure 5.6 shows the cross sections used to calculate line intensities. The sensitivity of the line ratio method strongly depends on the difference in cross sections of the chosen transition lines (see section 4.3.4). In this light, one should not solely use the transitions in Figure 5.6b to calculate line ratios (i.e., lines $I_{3}-I_{5}$ in Table 5.2), but better include a transition from Figure 5.6c (lines $I_{1}$ and $I_{2}$ in Table 5.2) into the calculation. This is illustrated in Figure 5.7 where one can observe that the ratio $I_{1} / I_{4}$ is a much steeper function of the electron energy than the ratio $I_{4} / I_{5}$. Hence, ratio $I_{1} / I_{4}$ is more sensitive to changes in the EEDF than $I_{4} / I_{5}$. Ratio $I_{1} / I_{4}$ corresponds to ionized argon $\mathrm{Ar}^{+}$and excited argon Ar. The $\mathrm{Ar}^{*}$ and $\mathrm{Ar}^{+*}$ emissions are characteristic to the shape of EEDF in the region above $14 \mathrm{eV}$.

Several calculated and measured line ratios at different pressures are plotted in Figure 5.8. A quick look at this figure immediately reveals a large difference in line ratios calculated from the entire-range experimental EEDF and the corresponding MB-part only. Generally, the ratios calculated from the MB-part are 1-2 orders of magnitude smaller than the ones calculated from 


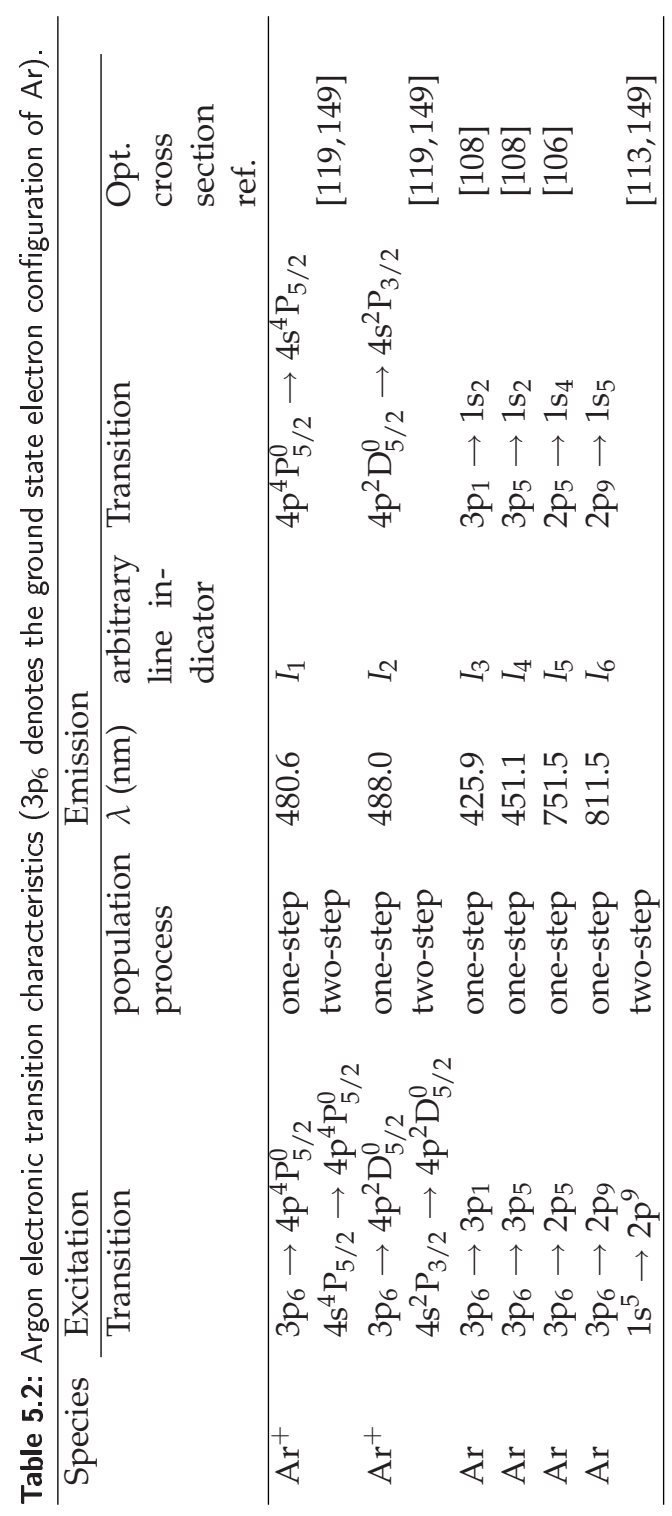



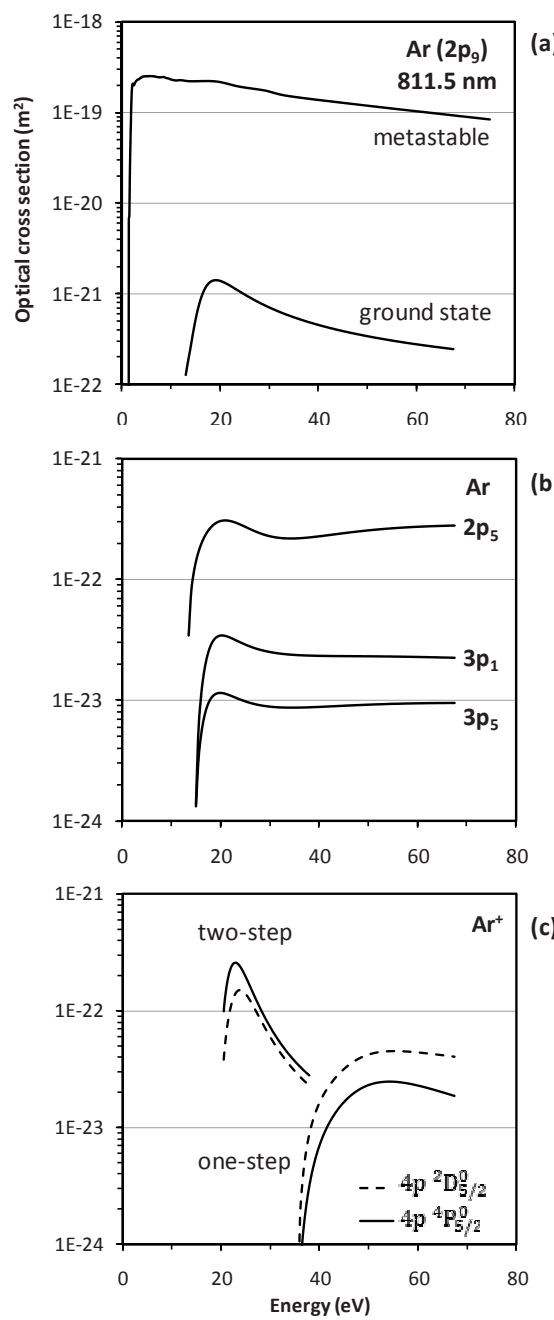

Figure 5.6: Optical cross sections of the chosen transitions. Data was taken from (a) $[113,149]$; (b) $[106,108]$; (c) $[119,149]$; (see Table 5.2 for details). 


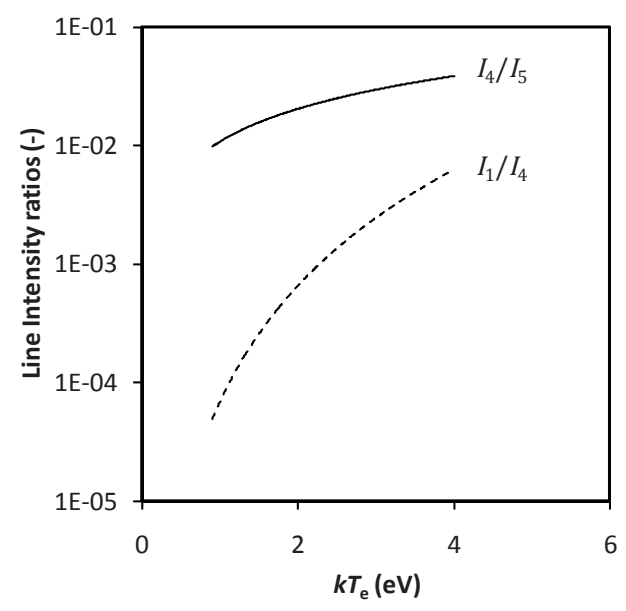

Figure 5.7: Typical Line intensity ratios as a function of the characteristic energy of a Maxwell-Boltzmann distribution. See Table 5.2 for details. Line ratios were calculated by equation (A.6) in Appendix A.

the entire EEDF. As argued before, this can be explained by the contribution of the fast electrons in the entire-range EEDF. The excitation of $\mathrm{Ar}^{+}$increases when the density of fast electrons increases, and consequently the photon decay rate increases. This results in a larger $\mathrm{Ar}^{+}$line intensity and hence a larger ratio $I_{\mathrm{Ar}^{+}} / I_{\mathrm{Ar}}$. One can also observe that the ratios calculated from the MBparts decrease by increasing the total pressure from $1 \mathrm{~Pa}$ to 6 and $12 \mathrm{~Pa}$ ( $c f$. Figure 5.8a-c). This is mainly due to a decrease of the characteristic electron energy in relation with the pressure (i.e., $1.7 \mathrm{eV}$ at $1 \mathrm{~Pa}$; $1.2 \mathrm{eV}$ at $6 \mathrm{~Pa}$, and $0.9 \mathrm{eV}$ at $12 \mathrm{~Pa}$ ). Again, there are fewer electrons available to excite $\mathrm{Ar}^{+}$for a distribution with a lower characteristic electron energy. A decreasing ratio $I_{\mathrm{Ar}^{+}} / I_{\mathrm{Ar}}$ can also be observed when one compares the ratio calculated from the entire-range experimental EEDF at $1 \mathrm{~Pa}$ with the ratios at 6 or $12 \mathrm{~Pa}$. This is easily understood when one compares the measured EEDFs at these pressures: the fast electrons extend to much higher energies at $1 \mathrm{~Pa}$ than at 6 or $12 \mathrm{~Pa}$. Hence, their contribution to excitation is larger at $1 \mathrm{~Pa}$, which results in larger ratios.

The calculated ratios should be compared with the measured ratios to find out if (or which of) the calculations reflect(s) the plasma composition the best. It is immediately clear from Figure 5.8 that the calculations based on 

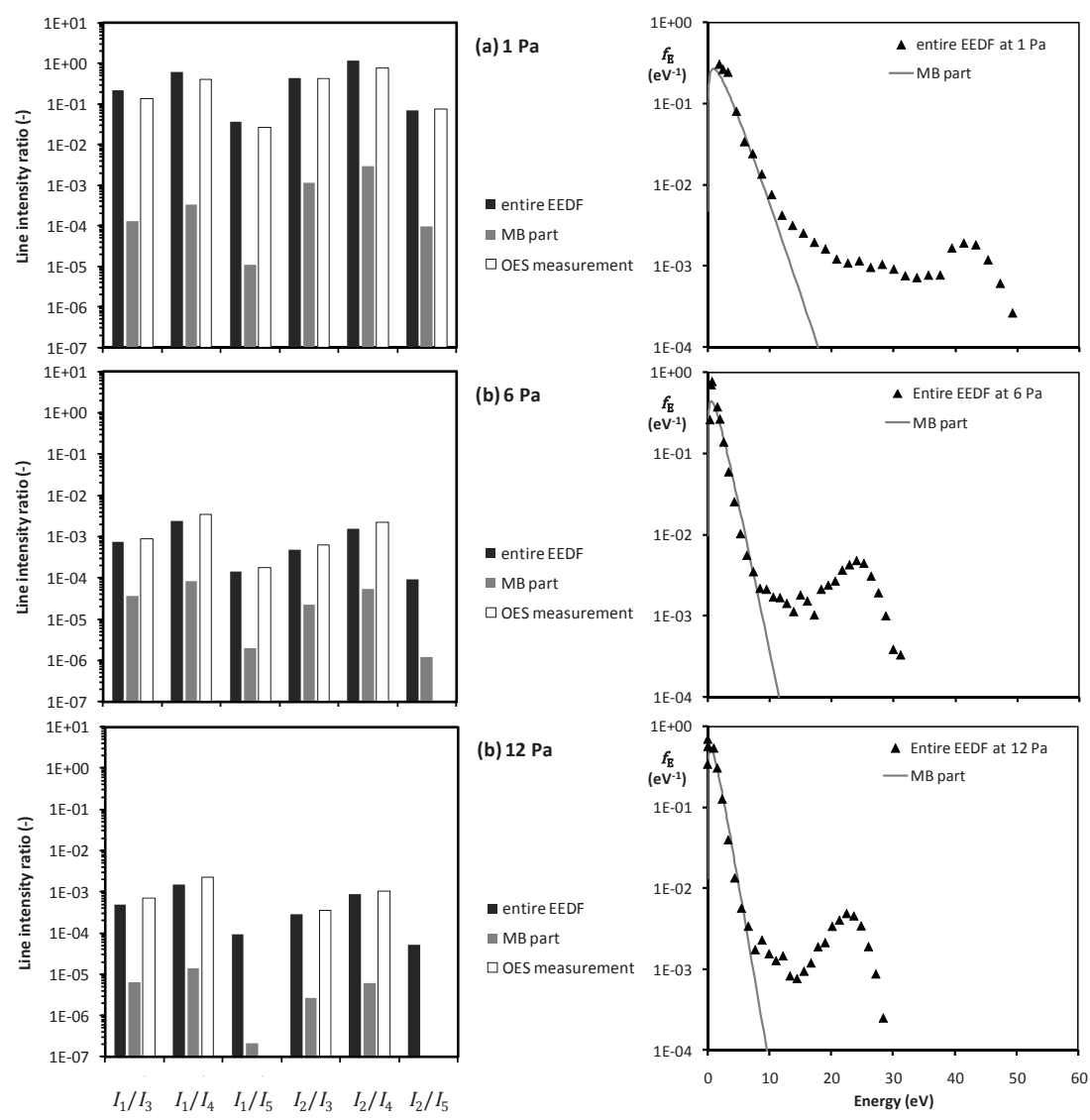

(b) $12 \mathrm{~Pa}$

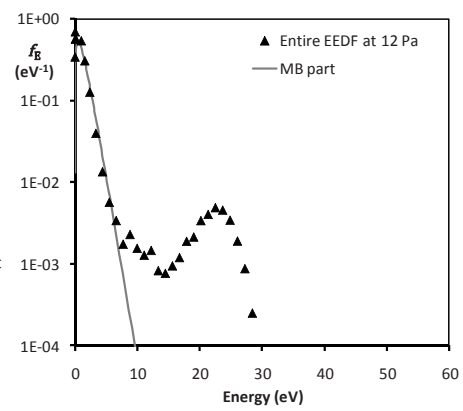

Figure 5.8: Calculated and measured line intensity ratios for several Ar lines. EEDFs presented at the right part of the figure were used as input for the calculations. See Table 5.2 for details. Line ratios were calculated by equation (A.6) in Appendix A. OES spectra and EEDFs were taken at $240 \mathrm{~mm}$. The calculated ratios include the entirerange experimental EEDF (i.e., MB part + high-energy tail) and the corresponding MB-part only. Ratios smaller than $10^{-4}$ could not be measured correctly; they fell outside the detector's linear range. 
the entire EEDF follow the measured ratios more closely than the calculations based on the MB-part only. The differences between the measured ratios and the calculations based on the entire EEDF are 15-40\%, whereas the measured and the MB-calculated ratios differ by orders of magnitude. An experimental error of $15-40 \%$ is actually surprisingly accurate considering the reported uncertainties in the cross sections ( $\leq 15 \%$, see references in Table 5.2). One can conclude that the EEDFs are measured correctly, including the fast-electron tail. The tail contributes largely to the plasma composition and ignoring it would cause strong disagreements between the actual plasma processes and the modeled ones.

\subsubsection{Estimate of the density of metastable atoms}

The line intensity ratio of the $811.5-\mathrm{nm}$ emission $2 \mathrm{p}_{9}$ level $\left(I_{6}\right.$ in Table 5.2$)$ could not be included in the EEDF analysis of the preceding section since this level is partly populated from the $1 \mathrm{~s}_{5}$ metastable level and the density of these metastables was unknown. Alternatively, the measured line intensity from this level can be used to estimate the density of these metastables, see B. The modeled line intensity ratio can be tuned to the measured one by choosing the proper $N_{\mathrm{Ar}\left(1 \mathrm{~s}_{5}\right)}$. This results in $N_{\mathrm{Ar}\left(1 \mathrm{~s}_{5}\right)}=4 \cdot 10^{15} \mathrm{~m}^{-3}$ at $1 \mathrm{~Pa}$. Unfortunately, the ratios could not be measured correctly at higher pressures, as they fell outside the detector's linear range (i.e., the intensity of the ionized argon line was too small with respect to the $811.5-\mathrm{nm}$ emission line).

\subsection{Ar- $\mathrm{N}_{2}, \mathrm{Ar}-\mathrm{N}_{2} \mathrm{O}$ and $\mathrm{Ar}-\mathrm{SiH}_{4}$ plasmas}

In this section, we demonstrate the influence of the addition of nitrogen and nitrous oxide on the EEDF. As seen in Figure 5.9, a small amount of $\mathrm{N}_{2}$ or $\mathrm{N}_{2} \mathrm{O}$, added to Ar plasma, decreases the total number of slow (MB) electrons. However, the tail of fast electrons remains unchanged. This is in agreement with our OES measurements in $\mathrm{Ar}-\mathrm{N}_{2} \mathrm{O}$ plasmas (see Figure 5.10), showing no change in line ratios for the same plasma compositions. The $\mathrm{Ar}^{+*}$ and $\mathrm{Ar}^{*}$ emissions are characteristic to the shape of EEDF in the region above $14 \mathrm{eV}$ $[48,128]$. This energy range, according to Figure 5.9 , is not significantly affected by the addition of $\mathrm{N}_{2}$ or $\mathrm{N}_{2} \mathrm{O}$.

However, the question arises whether EEDFs obtained for these plasmas are similar to those of plasmas with small additions of silane. Since direct Langmuir-probe measurements are not possible in silane-containing plasmas, line ratios were measured by OES only. The results are presented in Figure 5.10. Line ratios remain unaffected up to a 8 vol. \% of silane. One can conclude that 
the fast-electron tail of an argon plasma remained unchanged upon addition of 8 vol.\% of silane. It has however been calculated that the number of slow electrons increases when one adds silane to argon plasmas, and this effect is missed by the OES method. Pfau [150] calculated (based on MB-statistics and collision rate data) a two-times higher density of (slow) MB-electrons in a $\mathrm{Ar}-5 \% \mathrm{SiH}_{4}$ plasma compared to a pure argon plasma. Similar results were obtained by the calculations of Nagpal and Garscadden [151], and the analyses of Kurachi and Nakamura [152]. At this point, the reader should recall that the emission of the Ar 811.5-nm line stretches out to these slow electrons because this level is also populated by $1 \mathrm{~s}_{5}$-metastables (see section 4.3 .3 and B. Inserting this increased $N_{\mathrm{e}}$ into the calculation of the $\operatorname{Ar}\left(1 \mathrm{~s}_{5}\right)$ metastable state, while simultaneously keeping the number of fast electrons constant, one can estimate that the addition of 2 vol.\% of silane to argon plasma would increase $I_{6}$ by only $8 \%$. This increase was not measurable; probably due to the experimental error of the OES measurements, that was already $40 \%$. To be on the safe side, 0.8 vol. $\%$ was used as the highest silane fraction in this thesis. It increases the experimental error by only $3 \%$. Hence, the original full-range experimental EEDF obtained without silane was considered as valid for the silane-containing plasma as well. See B for details.

Summarizing our findings, we can conclude that adding silane to an argon plasma (at $1 \mathrm{~Pa}$ ) will not affect the fast-electron tail when the concentration is below 8 vol.\%, but, more importantly, will also not significantly affect the slow electrons when the density is kept below 2 vol.\%. This is an important result for chemical modeling, because actual electron densities and EEDFs obtained from $\operatorname{Ar}-\left(\mathrm{N}_{2}\right.$ or $\left.\mathrm{N}_{2} \mathrm{O}\right)$ plasmas can be used as input parameters to model electron-stimulated reactions in $\mathrm{Ar}-\left(\mathrm{N}_{2}\right.$ or $\left.\mathrm{N}_{2} \mathrm{O}\right)-\mathrm{SiH}_{4}$ plasmas. Donnelly et al. [153] reported that they added $1 \%$ each of the noble gases (i.e., $\mathrm{He}$, $\mathrm{Ne}, \mathrm{Ar}, \mathrm{Kr}, \mathrm{Xe} ; 5 \%$ in total) to determine $k T_{\mathrm{e}}$ in $\mathrm{Cl}_{2}$ plasmas at a pressure of 2.7 StateplacePa. Desai et al. [126] reported that Langmuir probe and OES data agreed with each other for Ar plasmas up to a pressure of $20 \mathrm{~Pa}$; while Kang et al. did the same for pressures up to $12 \mathrm{~Pa}$ [84]. These pressure range and precursor fractions are in the same range as the results presented in this work.

\subsection{Conclusions}

The electron energy distribution function is one of the most important characteristics of plasma reactors. In modern high-density plasma reactors operating at very low gas pressures, EEDFs can strongly deviate from the widely assumed 


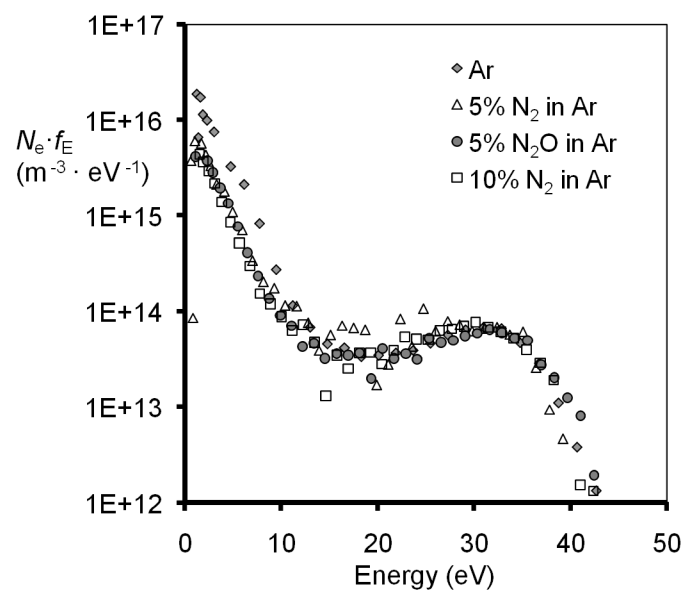

Figure 5.9: EEDFs for $\mathrm{Ar}-\mathrm{N}_{2}$ plasma and $\mathrm{Ar}-\mathrm{N}_{2} \mathrm{O}$ plasma (Probe position $80 \mathrm{~mm}$; ICP power $300 \mathrm{~W} ; p=1 \mathrm{~Pa}$ ).

Maxwell-Boltzmann distribution. This deviation will result in significant modeling and interpretation errors.

We presented our results on a series of Langmuir-probe measurements which were carried out to characterize a remote ICPECVD system. We measured EEDFs and mean electron temperatures in non-depositing $\mathrm{Ar}-\mathrm{N}_{2}$ and $\mathrm{Ar}-\mathrm{N}_{2} \mathrm{O}$ plasmas. The EEDFs exhibited two different components: a large part of slow electrons, following Maxwell-Boltzmann energy distributions, and a smaller part (about $0.5 \%$ of total electron density) of fast electrons in the energy range from 20 to $50 \mathrm{eV}$, strongly deviating from Maxwell-Boltzmann distribution. In spite of their low concentration, these fast electrons were of prime importance for plasma processes.

Characteristic energy of the slow electrons decreases with increasing gas pressure; from $k T_{\mathrm{e}}=1.8 \mathrm{eV}$ at $1 \mathrm{~Pa}$ to $k T_{\mathrm{e}}=0.9 \mathrm{eV}$ at $12 \mathrm{~Pa}$. The fast electron tail shifts to lower energies when the pressure is increased (i.e., from $33 \mathrm{eV}$ at $1 \mathrm{~Pa}$ to $22 \mathrm{eV}$ at $12 \mathrm{~Pa}$ ). Similar effects can be observed when an external axial magnetic field is applied to the plasma; $k T_{\mathrm{e}}$ of slow electrons decreases from $1.7 \mathrm{eV}$ to $1.4 \mathrm{eV}$ (at $1 \mathrm{~Pa}$ ) when a magnetic field of $9.5 \mu \mathrm{T}$ is applied, and the fast electron tail shifts from $32 \mathrm{eV}$ to $22 \mathrm{eV}$.

With respect to the EEDF at $1 \mathrm{~Pa}$, it appears that the tail of fast electrons shifts to higher energies with an increasing distance from the ICP-source. At 


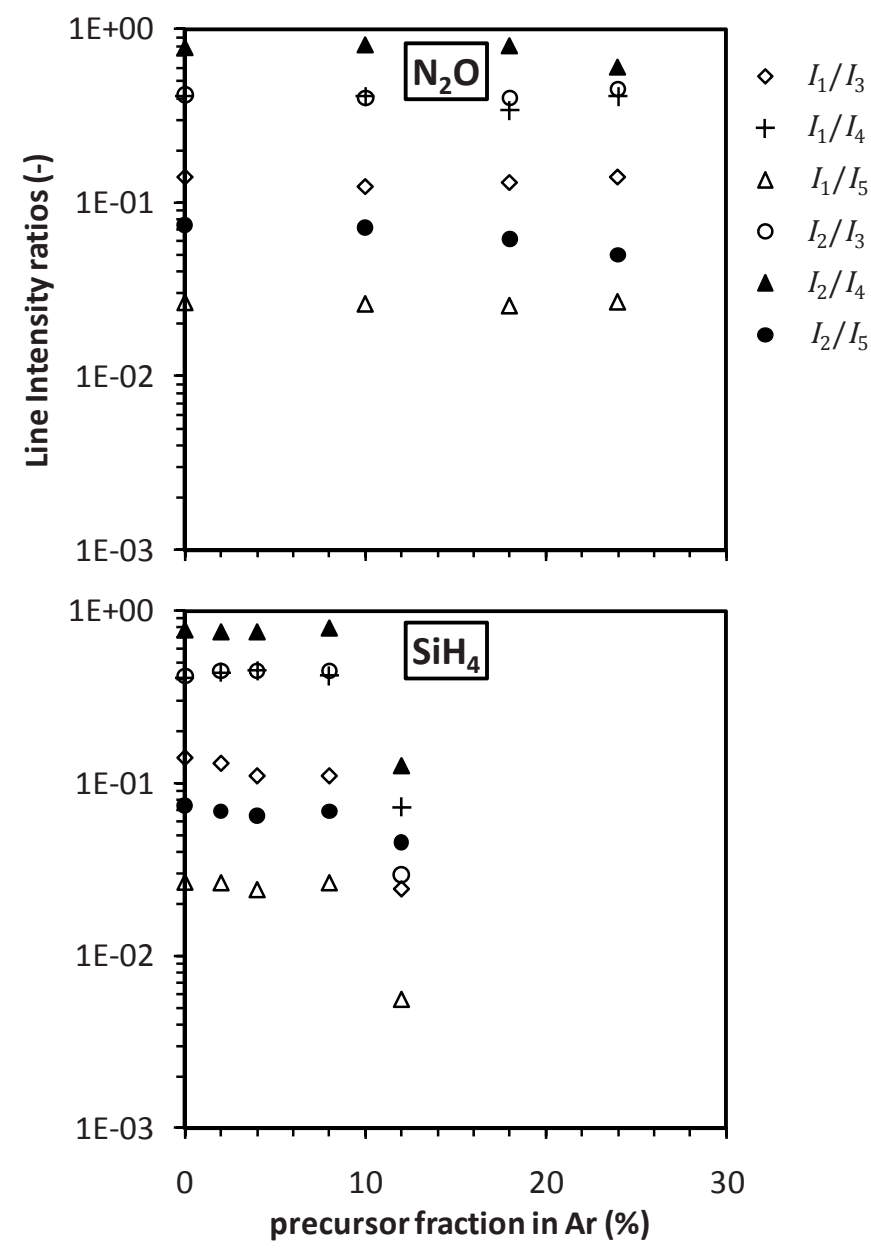

Figure 5.10: Line intensity ratios measured by OES for argon plasmas with several precursor gases at $1 \mathrm{~Pa}$. ICP power $300 \mathrm{~W}$. See Table 5.2 for details on the transitions $I_{1}-I_{5}$. 
higher pressures this shift to higher energies decreases (6 Pa), or disappears (12 $\mathrm{Pa}$ ). This results in pressure range of 1-6 $\mathrm{Pa}$ in which the ion bombardment can be controlled by choosing a proper position of the chuck rather then by applying a seperate rf bias.

We further developed a route to obtain EEDFs for depositing plasmas with silane, using a combination of the Langmuir-probe method and optical emission spectroscopy. Line intensities measured by OES were mainly related to the fast electrons, and therefore reflected their energies. Our method comprised three steps. First, EEDFs of Ar plasmas were measured by a Langmuir probe, while line intensity ratios were obtained by OES under the same conditions. Second, we verified that, with small additions of $\mathrm{N}_{2}$ and $\mathrm{N}_{2} \mathrm{O}$, Langmuir probe and OES methods gave similar results. Third, small amounts of silane were added to $\mathrm{Ar}, \mathrm{Ar}-\mathrm{N}_{2}$, and $\mathrm{Ar}-\mathrm{N}_{2} \mathrm{O}$ plasmas, and the change in line ratios was monitored by OES only. Below a certain "threshold" concentration of silane, it is possible to use the EEDFs measured in the plasmas without silane. This allows chemical modeling of depositing plasmas, even though a Langmuir probe measurement cannot be carried out in depositing plasmas. 


\section{Utilization of the measured plasma parameters}

\subsection{Introduction}

The purpose of this chapter is to demonstrate that modeling of gas-phase reactions can contribute to understand properties of $\mathrm{SiO}_{2}$ films deposited at different deposition conditions in $\mathrm{Ar}-\mathrm{N}_{2} \mathrm{O}-\mathrm{SiH}_{4}$ plasmas. The plasma composition will be calculated for several total gas pressures and silane fractions. Relations will be drawn between plasma composition and the expected deposition rate, and the electrical properties of the films. This chapter summarizes the work published in [154-156].

To model electron-stimulated reactions in plasma, one should know the reaction cross sections, partial pressures of reactants, electron densities and electron energy distribution functions (EEDF). Langmuir-probe measurements provided the EEDF and electron densities. Langmuir-probe measurements were shown in the preceding chapter. It was demonstrated that only a correctly measured EEDF could explain line intensity ratios as measured with OES. Intuitively, the same will hold for other electron-stimulated reactions in $\mathrm{Ar}-\mathrm{N}_{2} \mathrm{O}-$ $\mathrm{SiH}_{4}$ plasma.

\subsection{Modeling the plasma composition}

The model is introduced in Figure 6.1. A choice was made from the reactions known from literature. The high-pressure regime was not considered because that is normally not suitable for deposition of high-quality materials (due to the 


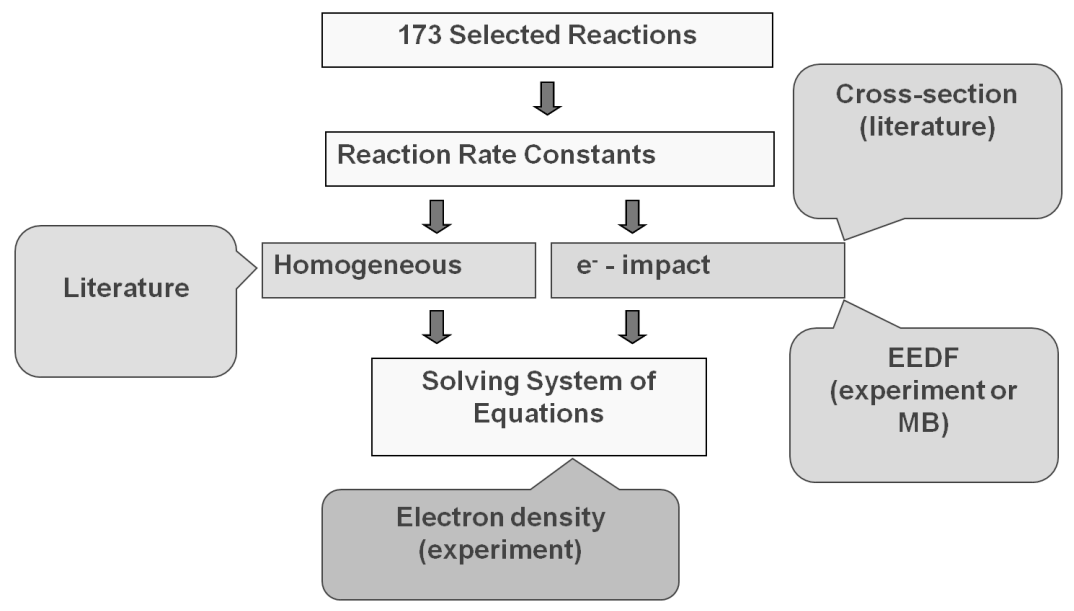

Figure 6.1: The chemical model.

gas-phase nucleation). Only electron-impact and secondary homogeneous reactions with reaction rates larger than $10^{-16} \mathrm{~m}^{3} / \mathrm{s}$ were selected. The densities of particles were obtained as a function of the reaction time by resolving the system of non-linear differential equations, $\mathrm{d} C_{\mathrm{i}} / \mathrm{d} t$. The reaction rate constants of electron-impact reactions were calculated by equation (3.35). It was already clear from section 4.3.3 that plasma excitation could not be described solely by electron-impact reactions. The same holds for other reactions in plasma. That is why secondary homogeneous reactions were also considered. The selected reactions have been summarized in [155] (which also shows the original references), and are introduced shortly in the next sections.

\subsubsection{Reactions involving $\mathrm{SiH}_{4}$ and $\mathrm{H}_{2}$}

Electron-impact reactions involving silicon and/or hydrogen-containing species are numerous. Hydrogen is not directly fed into our reactor, but species are formed by dissociation of $\mathrm{SiH}_{4}$. Electron-impact reactions can be divided in six categories:

1. Dissociative excitation of $\mathrm{SiH}_{x}(x=1-4)$ (10 reactions),

$$
\mathrm{e}+\mathrm{SiH}_{x} \rightarrow \mathrm{SiH}_{x-1}+\mathrm{H}_{y}+\mathrm{e} \quad(y=1,2)
$$

2. Dissociative ionization of $\mathrm{SiH}_{x}(x=1-4)$ (19 reactions),

$$
\mathrm{e}+\mathrm{SiH}_{4} \rightarrow \mathrm{SiH}_{x-1}^{+}+\mathrm{H}_{y}+2 \mathrm{e} \quad(y=1,2)
$$


3. Dissociative excitation of $\mathrm{SiH}_{\mathrm{x}}^{+}(x=1-3)$ (10 reactions),

$$
\mathrm{e}+\mathrm{SiH}_{x}^{+} \rightarrow \mathrm{SiH}_{\mathrm{x}-1}^{+}+\mathrm{H}_{y}+\mathrm{e} \quad(y=1,2)
$$

4. Dissociative recombination of $\mathrm{SiH}_{x}^{+}$(8 reactions),

$$
\mathrm{e}+\mathrm{SiH}_{x}^{+} \rightarrow \mathrm{SiH}_{x-1}+\mathrm{H}_{y}+2 \mathrm{e} \quad(y=1,2)
$$

5. Electron collisions with hydrogen (6 reactions),

$$
\mathrm{e}+\mathrm{H}_{2} \rightarrow \mathrm{H}+\mathrm{H}^{*}+\mathrm{e}
$$

6. Dissociative excitation of disilane and trisilane (3 reactions).

Secondary homogeneous reactions involve:

7. Charge exchange of $\mathrm{SiH}_{x}$ with $\mathrm{H}^{+}$(8 reactions),

8. Other homogeneous reactions (47 reactions), including e.g., dissociative recombination of $\mathrm{Si}_{2} \mathrm{H}_{1-5}^{+}$species.

\subsubsection{Reactions between $\mathrm{SiH}_{4}$ and $\mathrm{Ar}$}

Argon metastables and argon ions can also react with silane. Three reactions are considered:

9. Dissociative excitation of $\mathrm{SiH}_{4}$ by metastable Ar,

$$
\mathrm{Ar}^{*}+\mathrm{SiH}_{4} \rightarrow \mathrm{SiH}_{2}+2 \mathrm{H}+\mathrm{Ar}
$$

10. Dissociative ionization of $\mathrm{SiH}_{4}$ by $\mathrm{Ar}^{+}$(2 reactions),

$$
\begin{aligned}
& \mathrm{Ar}^{+}+\mathrm{SiH}_{4} \rightarrow \mathrm{SiH}_{3}^{+}+\mathrm{H}+\mathrm{Ar} \\
& \mathrm{Ar}^{+}+\mathrm{SiH}_{4} \rightarrow \mathrm{SiH}_{2}^{+}+2 \mathrm{H}+\mathrm{Ar}
\end{aligned}
$$

\subsubsection{Reactions involving $\mathrm{N}_{2} \mathrm{O}, \mathrm{N}_{2}, \mathrm{O}_{2}$, and $\mathrm{Ar}$}

These reactions include:

11. Dissociative excitation of $\mathrm{N}_{2} \mathrm{O}, \mathrm{N}_{2}$ and $\mathrm{O}_{2}$ species (6 reactions), e.g.,

$$
\mathrm{e}+\mathrm{N}_{2} \mathrm{O} \rightarrow \mathrm{N}_{2}+\mathrm{O}\left({ }^{3} \mathrm{P}\right)+\mathrm{e}
$$

12. Homogeneous reactions (17 reactions), e.g.,

$$
\begin{aligned}
& \mathrm{Ar}^{+}+\mathrm{N}_{2} \rightarrow \mathrm{N}_{2}^{+}+\mathrm{Ar} \\
& \mathrm{Ar}^{*}+\mathrm{O}_{2} \rightarrow \mathrm{O}+\mathrm{O}+\mathrm{Ar}
\end{aligned}
$$




\subsubsection{Reactions involving $\mathrm{SiH}_{4}$, and $\mathrm{N}_{2} \mathrm{O}$}

For depositing plasmas, 7 homogeneous reactions between $\mathrm{SiH}_{x}(x=0-4)$ species and $\mathrm{N}, \mathrm{NH}$ or $\mathrm{NH}_{2}$ radicals, and 21 homogeneous reactions between various $\mathrm{Si}$ - and oxygen-containing species were considered.

Based on a set of 173 reactions in total, densities of 43 reactive species were modeled, including $\mathrm{SiH}_{x}$ radicals, and $\mathrm{SiH}_{x}^{+}(x=0-3)$ ions, polysilanes, $\mathrm{SiO}, \mathrm{SiN}, \mathrm{SiH}_{3} \mathrm{O}, \mathrm{SiH}_{2} \mathrm{O}, \mathrm{HSiO}$, etc., as well as atomic hydrogen, nitrogen and oxygen. As a practical application, we used our simulations to predict (in terms of $\mathrm{SiH}_{x}$ and $\mathrm{H}$ concentration) the influence of $\mathrm{SiH}_{4} / \mathrm{N}_{2} \mathrm{O}$ gas-flow ratio and total gas pressure on film electrical properties. See [155] for details and more references therein.

\subsection{Typical modeling conditions}

Boundary conditions of chemical modeling were set to match the process conditions mentioned in section 4.1.5. On top of that, the silane concentration had to be below $2 \mathrm{vol} \%$, such that it did not influence the EEDF (see section 5.3). We focused on the effects of total pressure and silane fraction on deposition kinetics and film properties.

Briefly, argon was used as a carrier gas, the process pressure ranged from 1 to $6 \mathrm{~Pa}$, while the total flow was fixed at $244 \mathrm{sccm}$. A flow of $44 \mathrm{sccm}$ of nitrous oxide $\left(\mathrm{N}_{2} \mathrm{O}\right)$ was added to the carrier gas in the plasma zone, while 100 or 10 sccm of $\mathrm{Ar}-\mathrm{SiH}_{4}$ mixture (2\% silane $\left(\mathrm{SiH}_{4}\right)$ in argon) was added downstream of the plasma. This resulted in an $\mathrm{N}_{2} \mathrm{O}$ fraction of 18 volume $\%$ and in a $\mathrm{SiH}_{4}$ fraction of 0.8 or $0.08 \%$, respectively.

\subsection{Impact of EEDF on silane-based plasmas}

Comparing the calculated (see equation (3.35)) reaction rate constants listed in Table 6.1, one can notice a strong impact of the used electron energy distribution function on the rate constants. When the (experimentally measured) entire-range EEDF is applied, the calculated constants are on 1-5 orders of magnitude higher compared to the use of the corresponding MB-parts only (cf. Figure 5.2), i.e., neglecting the high-energy tail. This result is completely in line with the differences in calculated line ratios between the two EEDFs ( $c f$. Figure 5.8). The entire range EEDF clearly enables much faster electron-impact reactions compared to the MB-only EEDF, which strongly affects the densities of the calculated species in plasma. This is illustrated in Figure 6.2, where the 


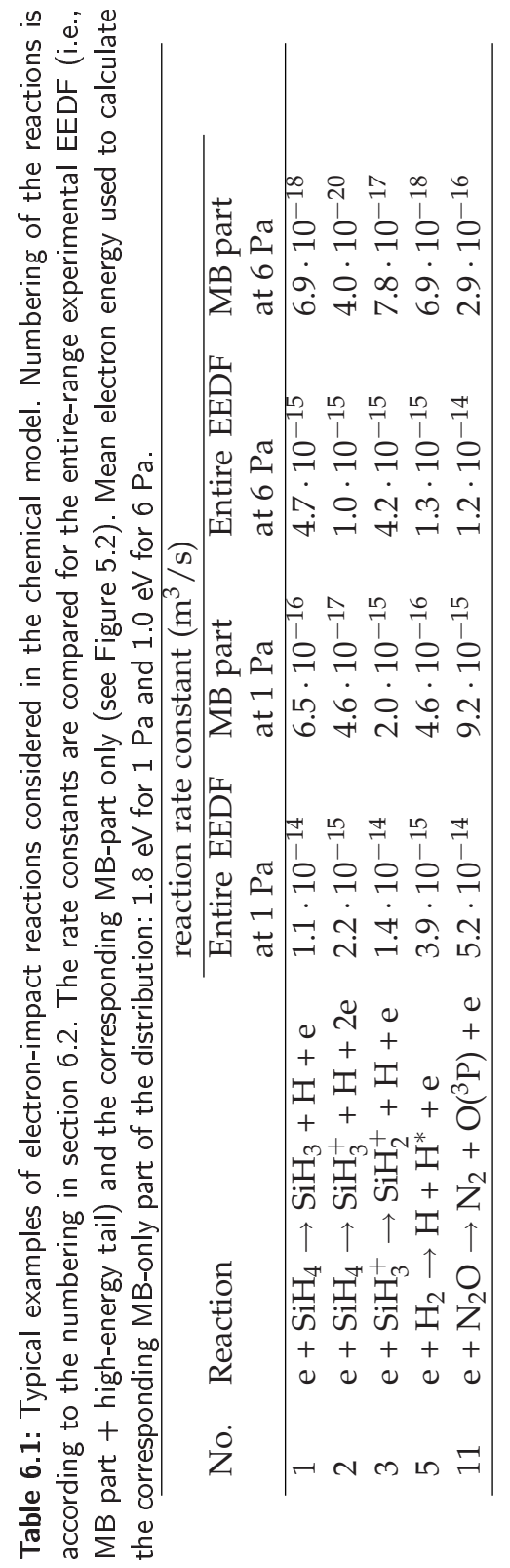


densities of several silicon species and hydrogen are plotted as a function of time. One can observe from this figure that silane decays very fast in our plasmas due to the presence of (high-energy) tail electrons. After 0.3 seconds of reaction time, silane is totally converted to atomic $\mathrm{Si}$ an $\mathrm{H}$ radicals at a initial silane fraction of $0.08 \%$, whereas at an initial silane fraction of $0.8 \%, \mathrm{SiH}_{2}$ and $\mathrm{SiH}$ reach significantly high densities at reaction times of $0.4 \mathrm{~s}$ and longer. Clearly, reaction time and initial silane fraction are parameters that create different plasma compositions, and hence deposition kinetics and layer qualities. The calculation of the reaction time is explained below.

The reaction time is determined by the residence time, $\tau$, of a gas in the system, which is a function of the volume of the chamber, $V$, (liter) and the pumping speed, $S$, (liter $\cdot \mathrm{s}^{-1}$ ) [34]. Since $S$ is not measured directly, we use instead:

$$
\tau=\frac{V}{S}=\frac{p V}{p S}=\frac{p V}{Q},
$$

where $p$ is the total pressure, and $Q$ is the throughput (in Torr $\cdot$ liter $\cdot \mathrm{s}^{-1}$ ). $Q$ is alternatively known as the flow rate, particularly when referring to the inlet rate of a reaction gas. The residence time is found to be $1 \mathrm{~s}$, for the conditions in Figure 6.2 ( $p=6 \mathrm{~Pa}$ (45 mTorr); $Q=244 \mathrm{sccm}=3.1$ Torr $\cdot$ liter $\cdot \mathrm{s}^{-1}$, and $V=681$ (see section 4.1). While the inlet rate was fixed, the pressure was set by throttling the pumping speed. Finally, the density of species can be found at the calculated $\tau$.

Figure 6.3 shows the calculated densities, based on the entire-range EEDF. For a $\mathrm{SiH}_{4}$-fraction of 0.08 volume\%, the densities of atomic $\mathrm{Si}$ and $\mathrm{H}$ are dominant over the entire pressure range. At a $\mathrm{SiH}_{4}$-fraction of 0.8 volume $\%$, the densities of atomic $\mathrm{Si}$ and $\mathrm{H}$ are dominant at $1 \mathrm{~Pa}$, while $\mathrm{SiH}_{3}, \mathrm{SiH}_{2}, \mathrm{SiH}$, and $\mathrm{Si}$ are dominating precursors at $6 \mathrm{~Pa}$. The density of atomic $\mathrm{H}$ is significantly lower at $6 \mathrm{~Pa}$ compared with $1 \mathrm{~Pa}$. In the case of using the MB-only EEDF, the simulations indicate a less strong dependence of plasma composition in relation with pressure, as depicted in Figure 6.4. One observes only subtle changes in the (relative) densities of the several Si species if one compares the densities at 1 and $6 \mathrm{~Pa}$ for the silane fraction of $0.8 \%$.

Oxygen radials $\left(\mathrm{O}\left({ }^{3} \mathrm{P}\right)\right.$ and $\left.\mathrm{O}\left({ }^{1} \mathrm{D}\right)\right)$ are important deposition precursor as well. Their total density is calculated to be about $4 \cdot 10^{19} \mathrm{~m}^{3}$ at all process conditions of Figure 6.3 [156]. This leads to a density ratio of atomic oxygen to silicon-species of $10-20$ at a silane fraction of $0.8 \%$, and of 200 at $0.08 \%$ silane. This will result in a flux of atomic oxygen towards the substrate that is 10-200 times higher than the flux of $\mathrm{Si}$ species.

Also volatile $\mathrm{SiO}$ monoxide can reach appreciable densities but is not 
included into the considerations, since the reactivity of $\mathrm{SiO}$ with the surface of a depositing silicon oxide film has been measured to be near zero for surface temperatures of $25-500^{\circ} \mathrm{C}$ [157].

\subsection{Predicting film properties by modeling}

A few predictions concerning layer properties of deposited $\mathrm{SiO}_{2}$ can be made from the modeled plasma composition. First of all, the density of the several silane radicals determines the deposition rate and oxide quality, since the atomic $\mathrm{O}$ density is constant over all process conditions. The film mass density, and the concentration of charge-trapping centers (e.g. Si dangling bonds) can be considered in relation with relative plasma-densities of atomic hydrogen, Si-containing radicals and their sticking probabilities.

The mass density of the film is influenced by sticking probabilities of dominating Si-containing radicals. It is known that $\mathrm{SiH}_{3}$ radicals have the longest lifetime before they react at the surface. They have a smaller sticking probability $\left(0.28\right.$ below $400^{\circ} \mathrm{C}$ according to [158]) and can therefore experience several adsorption sites before reacting $[159,160]$. This leads to a conformal coverage and appropriate film density. In contrast, the sticking probability of Si radicals is close to unity (on an $a-\mathrm{Si}: \mathrm{H}$ surface) even at room temperature [161,162]. As they can react on contact, they are not able to migrate over the film; their dominant flux to the surface will lead to the formation of films with less appropriate chemical network, resulting in a lower mass density.

The leakage current of the oxide is strongly influenced by the material density and the concentration of charge-trapping centers. A lower density at the same physical thickness is caused by nano- or micro-voids and will result in a higher leakage current. Traps can also enhance this current. The fixed positive oxide charge is related to the concentration of charge-trapping centers. These centers can appear after ionizing for example Si dangling bonds and forming $\mathrm{Si}^{+}$ions.

It is known that atomic hydrogen passivates the traps when e.g. an aluminum gate is present $[163,164]$. In this light, any ambient, containing $\mathrm{H}$ atoms can provide the required passivation of the traps by forming the relatively stable $\equiv \mathrm{Si}-\mathrm{H}$ and $\equiv \mathrm{Si}-\mathrm{OH}$ bonds. However, an exposure to $\mathrm{H}$-containing plasma can also have the opposite effect if the so-called atomic hydrogen abstraction dominates $[165,166]$. Hydrogen abstraction is an interaction of the adsorbed (chemically bonded) $\mathrm{H}$ atom with a free $\mathrm{H}$ atom impinging on the surface, which results in the $\mathrm{H}_{2}$ desorption and thus leaving an unsaturated bond, i.e. a trap. A high $\mathrm{H}$-density in plasma can therefore have a negative effect on the 

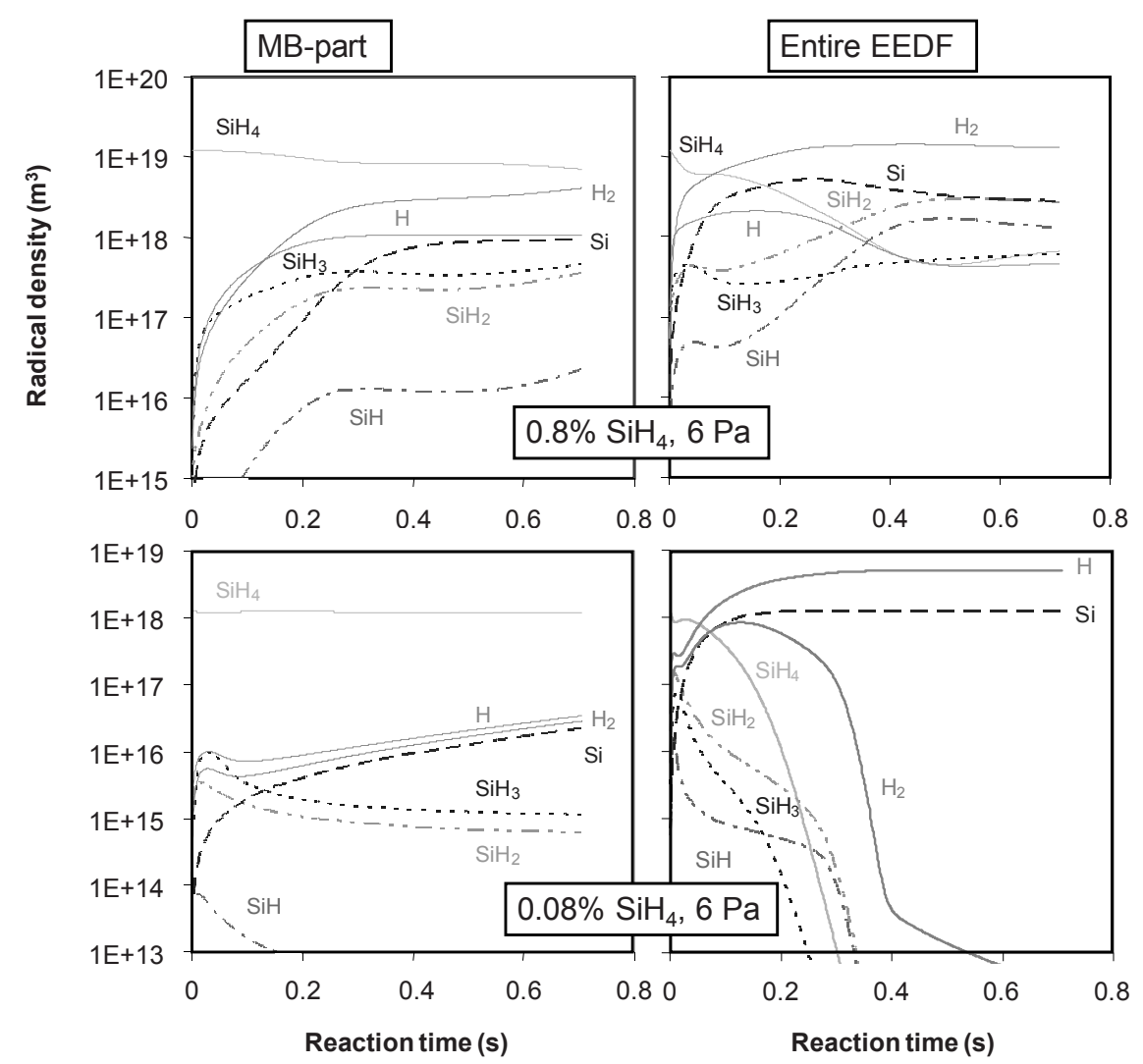

Figure 6.2: Densities of several silicon and hydrogen species as a function of time at typical process conditions. An $\mathrm{N}_{2} \mathrm{O}$ fraction of 18 vol.\% was used at all process conditions. Only some species are displayed for reasons of clarity (species were taken from section 6.2.1). 


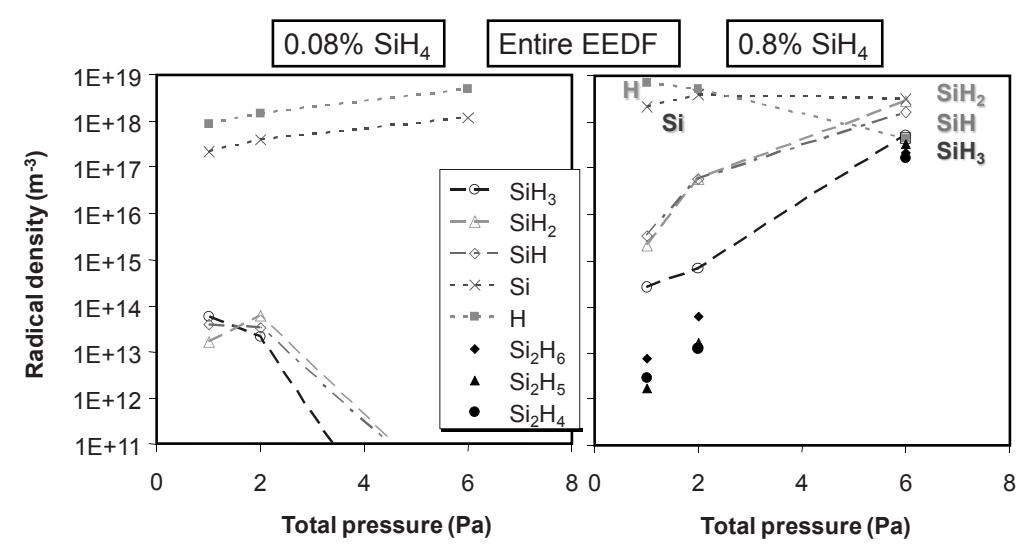

Figure 6.3: Radical densities, calculations based on residence time $\tau$ (eq. (6.1)), experimental EEDFs and electron concentrations for $\mathrm{Ar}-\mathrm{N}_{2} \mathrm{O}(18 \%)$ plasmas. Data taken from [155].

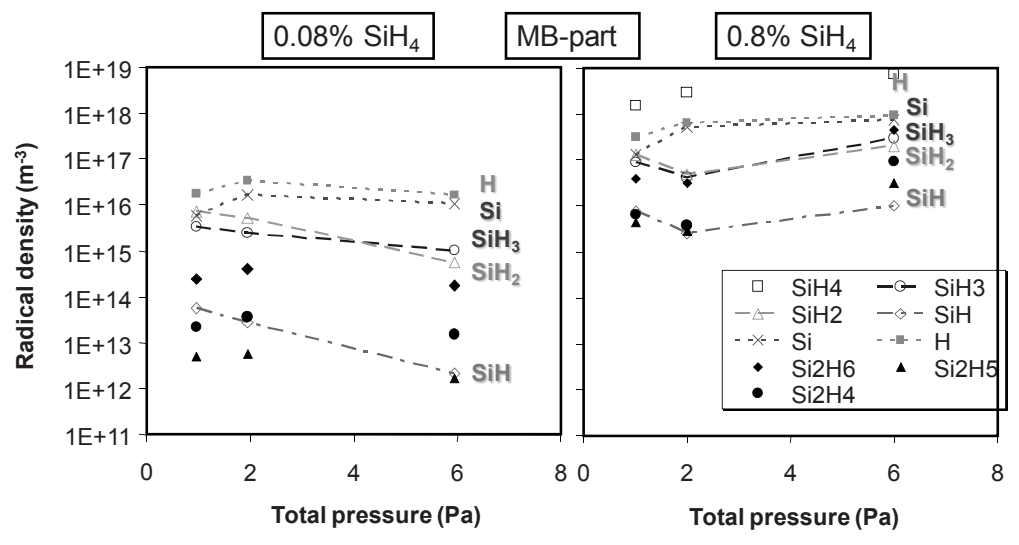

Figure 6.4: Radical densities, calculations based on residence time $\tau$ (eq. (6.1)), MB EEDFs and electron concentrations for $\mathrm{Ar}-\mathrm{N}_{2} \mathrm{O}(18 \%)$ plasmas. Data taken from [155]. 
trap passivation and increase the amount of $\equiv \mathrm{Si} \cdot$ dangling bonds and, thus, the positive charge in the oxide. Besides passivation and abstraction, atomic hydrogen can also etch silicon at low substrate temperatures $[167,168]$. Too much hydrogen might therefore lower the deposition rate.

\subsubsection{Silane fraction of $\mathbf{0 . 8} \%$}

Atomic silicon (sticking probability near unity) strongly dominates at $1-2 \mathrm{~Pa}$ (Figure 6.3, right). Due to the changing plasma conditions, the Si density slightly decreases at $6 \mathrm{~Pa}$ compared to $2 \mathrm{~Pa}$. At $6 \mathrm{~Pa}$, however, densities of the other $\mathrm{SiH}_{x}$ radicals increase dramatically and approach the level of atomic Si. A combination of both effects will lead to comparable deposition rates at 1, 2, and $6 \mathrm{~Pa}$.

Because of the lower relative density of atomic Si in plasma at $6 \mathrm{~Pa}$, the formation of electrically-better films is expected. This is due to a better surface migration of the other dominating $\mathrm{SiH}_{x}$ species and a lower flux of atomic $\mathrm{Si}$ towards the film surface, as discussed above. This will result the growth of denser films with lower leakage currents at $6 \mathrm{~Pa}$ (see Table 6.2).

The calculated density of atomic hydrogen at $6 \mathrm{~Pa}\left(4.4 \times 10^{17} \mathrm{~m}^{-3}\right)$ is significantly lower than that at $1 \mathrm{~Pa}\left(7.4 \times 10^{18} \mathrm{~m}^{-3}\right.$, see Figure 6.3 , right). This might cause a change in the balance between trap passivation and trap creation, as well as between Si deposition and Si etching. One could expect that the lower hydrogen density at $6 \mathrm{~Pa}$ will create films with less traps and less oxide charge. On the contrary, it could also be that these hydrogen densities still favor passivation mechanisms. If that is true, the films deposited at $1 \mathrm{~Pa}$ should show less traps and less oxide charge. This shows the limits of the predictive value of gas-phase modeling. To improve, surface reactions should be added.

A less strong relation between pressure and the plasma composition was found when MB-EEDFs were used. Figure 6.4 demonstrated only subtle changes in the (relative) densities of the several Si species at different pressures. Consequently, the predictive value of these simulations is lower in case of MBEEDFs.

\subsubsection{Silane fraction of $0.08 \%$}

Atomic silicon dominates in plasma for the entire pressure range (see Figure 6.3, left). At 1-2 $\mathrm{Pa}$, the other $\mathrm{SiH}_{x}$ species can still be noticed; however they disappear at $6 \mathrm{~Pa}$. Deposition in the entire pressure range is in this case mainly determined by the flux of atomic Si impinging on the film. This flux gradually increases with the pressure, this could cause a higher deposition rate in relation with pressure. Furthermore, it will also cause deposition of less-dense and electrically worse films at higher pressure. The best film quality is expected at 
Table 6.2: Predicted (based on modeling) electrical quality of $\mathrm{SiO}_{2}$ films deposited at several silane fractions and total pressures. An $\mathrm{N}_{2} \mathrm{O}$ fraction of 18 vol.\% was used at all process conditions. The adjectives "good" and "worse" may only be read in comparison with the film deposited at the same silane fraction but at a different total pressure.

\begin{tabular}{|c|c|c|}
\hline \multirow[b]{2}{*}{$1 \mathrm{~Pa}$} & $0.08 \% \mathrm{SiH}_{4}$ & $0.8 \% \mathrm{SiH}_{4}$ \\
\hline & "good" dielectric & "worse" dielectric \\
\hline \multirow{7}{*}{$6 \mathrm{~Pa}$} & mass density & mass density \\
\hline & traps & traps \\
\hline & leakage current & 喧 leakage current \\
\hline & "worse" dielectric & "good" dielectric \\
\hline & mass density & mass density \\
\hline & traps & traps \\
\hline & leakage current & leakage current \\
\hline
\end{tabular}

a pressure of $1 \mathrm{~Pa}$ due to the presence of small portions of the other $\mathrm{SiH}_{x}$ radicals. The calculated density of atomic hydrogen is lower at $1 \mathrm{~Pa}\left(8.5 \times 10^{17} \mathrm{~m}^{3}\right)$ than at $6 \mathrm{~Pa}\left(4.9 \times 10^{18} \mathrm{~m}^{-3}\right.$, see Figure 6.3). Again, this might cause a change in the balance between trap passivation and trap creation.

The predictions of film properties are summarized in Table 6.2. Silicon dioxide films were deposited at these process conditions, thereby relating to our work on plasma characterization and chemical modeling. The results are presented in Part II of this thesis. The aim is twofold: can we provide qualitative experimental proof for our modeling, and can we deposit silicon dioxide films with sound dielectric quality?

\subsection{Conclusions}

The composition in an $\mathrm{Ar}-\mathrm{SiH}_{4}-\mathrm{N}_{2} \mathrm{O}$ ICPECVD system was modeled. Both the experimental and MB EEDFs were used to calculate reaction rate constants for the electron-impact reactions. It was demonstrated that the use of an inappropriate EEDF, at first sight only slightly deviating form the experimental distribution, could cause strong disagreements between the actual plasma composition and the modeling.

Plasma densities of Si-containing radicals and atomic hydrogen were used to predict the electrical and physical properties of deposited $\mathrm{SiO}_{2}$ films. We explained their influence on deposition rate and mass density of the oxide, and on the concentration of charge-trapping centers in the material. 
PART II

THE SOLID STATE 


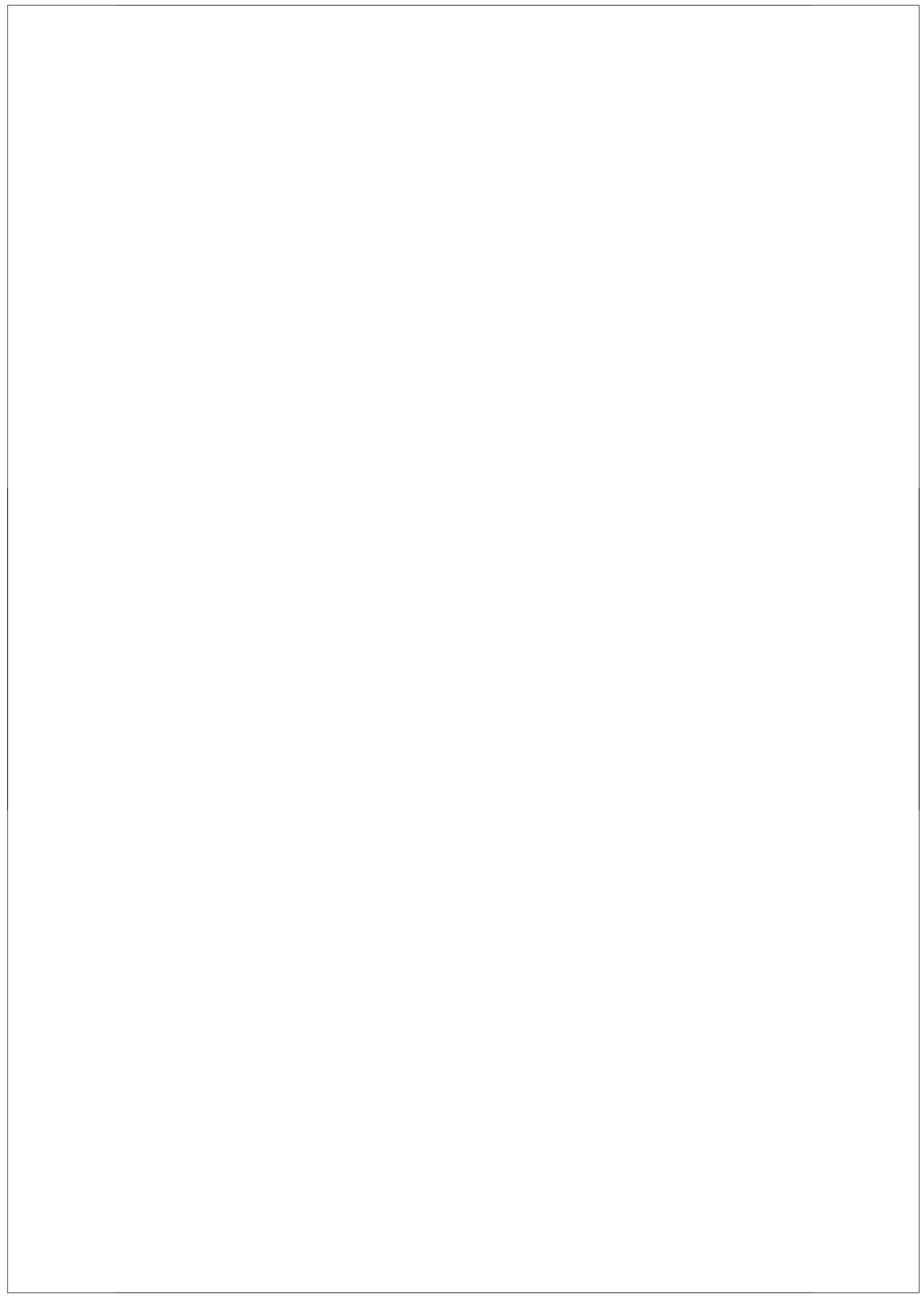




\section{Introduction}

Present-day semiconductor device manufacturing involves several hundred process steps. Many of these steps are carried out at high temperatures, between 400 and $1000^{\circ} \mathrm{C}$. Some of these steps are silicon oxidation, impurity activation, and thin film deposition. There is a great demand for lower temperature processing. A very wide range of electronic devices (e.g., thin film transistors (TFT), and non-volatile memory cells (NVM)) and MEMS/MOEMS can be improved significantly when lower temperatures $\left(20-400^{\circ} \mathrm{C}\right)$ are applied in the manufacturing process. One of the needs is to produce gate dielectrics at low substrate temperatures, as was already outlined in section 1.1. It was also sketched there that the temperature reduction has its price to some of the electrical and physical characteristics of the silicon dioxide layers: leakage currents, dielectric strength, fixed and mobile oxide charge, defect density at the interface with silicon, etch rate and index of refraction ( $c f$. Table 1.1. Selected properties of CVD and thermal silicon dioxide).

This chapter evaluates the electrical and physical characteristics of the silicon dioxide layers produced in our remote ICPECVD reactor at substrate temperatures of $150^{\circ} \mathrm{C}$. The characteristics of thermally grown silicon dioxide layers will act as the benchmark. Based on the preceding plasma characterization and chemical modeling, we could already predict the (electrical) properties of the deposited films (cf. Table 6.2). The validity of these predictions will be evaluated in this chapter.

Physical characteristics of thin $\mathrm{SiO}_{2}$ films can be obtained by a variety of $e x$ situ analytical techniques, such as scanning electron microscopy (SEM), transmission electron microscopy (TEM), infrared spectroscopy, spectroscopic 
ellipsometry (SE), X-ray reflectometry and diffraction (XRR and XRD), Rutherford backscattering spectroscopy (RBS), x-ray photoelectron spectroscopy (XPS), Auger electron spectroscopy (AES). These techniques provide information on step coverage, film thickness, microstructure, and atomic composition. However, recently more emphasis has been put on in situ diagnostics for online process monitoring and for control of the deposition process. In situ diagnostics allows, for example, fast determination of the incubation time in ALD processes [169]. For PECVD, one clear advantage of in situ monitoring, e.g. directly measuring the deposition rate will be illustrated in this thesis. In situ monitoring oxide growth by SE showed initially a non-linear fast growth regime that gradually transfered into a linear regime with a constant deposition rate. These two regimes would not have been distinguished without in situ monitoring. Infrared spectroscopy and ellipsometry can both be used for ex situ and in situ analysis.

Infrared spectroscopy has been employed in fundamental studies on the growth of $\mathrm{SiO}_{2}$ films and, in particular, provides the sensitivity to detect surface species [170]. Infrared spectroscopy can detect the minimal changes in transmission of the infrared light as caused by absorption of due to vibrations of certain groups of atoms in the film. The challenge is to obtain a sufficient signal-to-noise ratio on flat surfaces. Instead of infrared spectroscopy, spectroscopic ellipsometry was used in this study because of the much higher signalto-noise ratio (which makes it relatively more easy to implement), the wide photon energy range, and because it is more commonly applied to study films grown by physical and chemical vapor deposition techniques [171].

In addition to the monitoring and characterization of the PECVD process by (in situ) SE, ex situ spectroscopic analysis has been carried out to confirm the results obtained and, moreover, complement the film characterization of the deposited films with respect to thickness, step coverage (TEM), composition (XPS), and relevant electrical properties.

Charges in thin $\mathrm{SiO}_{2}$ films can be detected by various spectroscopic means: Photoluminescence (PL) [172] and cathodoluminescence (CL) [173] for example. CL has been employed to indicate defects in SiOx systems. Secondharmonic generation (SHG) allows for a contactless detection of electric fields in semiconductor space-charge regions arising from interfacial charge separation [174]. Electric field-induced SHG (EFISH) has been used to study charge trapping in the c-Si/ $\mathrm{SiO}_{2}$ system [175]. Infrared spectroscopy, internal photoemission, and electron spin resonance were applied to measure states at the $\mathrm{Si}-\mathrm{SiO}_{2}$ interface [176-178]. These spectroscopic techniques are relevant and valuable. They provide information on the (chemical) nature of the charges. 
However, since charges and traps derive their technological relevance from their electric activity, electrical characterization is applied in this thesis.

The charges in thin $\mathrm{SiO}_{2}$ films can be detected by measuring their field strengths towards the substrate $(C-V$ measurements) and towards the surface (Scanning Probe Microscopy $[179,180])$. C $-V$ measurements were applied in this thesis. These measurements are more sensitive to traps at the $\mathrm{Si}-\mathrm{SiO}_{2}$ interface than an SPM probe, and their results can be related more directly to the functioning of field-effect devices [181]. Furthermore, they can distinguish more easily between the different kinds of charges (mobile, ionic, interface, and fixed charge) $[182,183]$.

Besides the detection of charges, the dielectric needs to be analyzed on its leakage current, breakdown field and conduction mechanism. All these properties were extracted from current-voltage $(I-V)$ measurements. Other electrical techniques that were not used in this work are reviewed by Schroder [184]. 


\section{Experimental}

\subsection{Introduction}

This chapter contains an overview of various specific equipment used in this work. The PECVD deposition system was described in part I. A short description is given of the measurements and analysis techniques that are used to characterize the deposited films and to monitor the deposition process (i.e., spectroscopic ellipsometer, equipment and methods for the electrical measurements).

\subsection{Wafer preparation}

Films were deposited on H-terminated Si-wafers (n- or p-type $\langle 100\rangle$ ) that received standard cleaning. First, fuming nitric acid $\left(\mathrm{HNO}_{3} 100 \%\right)$ and boiling nitric acid $\left(\mathrm{NHO}_{3} 69 \%\right)$ were used in order to remove organic and metallic contaminants. The cleaning process was concluded by a $1 \%$ HF dip in order to remove the native silicon oxide. The substrates were rinsed with deionized water after all cleaning steps. The deposition process was monitored in situ using a J.A. Woollam M2000 spectroscopic ellipsometer (SE).

Additionally, metal-oxide-semiconductor (MOS) capacitors were implemented by sputtering $1-\mu \mathrm{m}$ Al over the oxide, followed by lithography and etching processes to define $0.06,0.1$, and $0.2 \mathrm{~mm}^{2}$ square capacitors. An $\mathrm{Al}$ layer was also sputtered on the backside of the Si wafer. Some wafers were subjected to post-metallization annealing (PMA) for $10 \mathrm{~min}$ at $400^{\circ} \mathrm{C}$ in humid, 


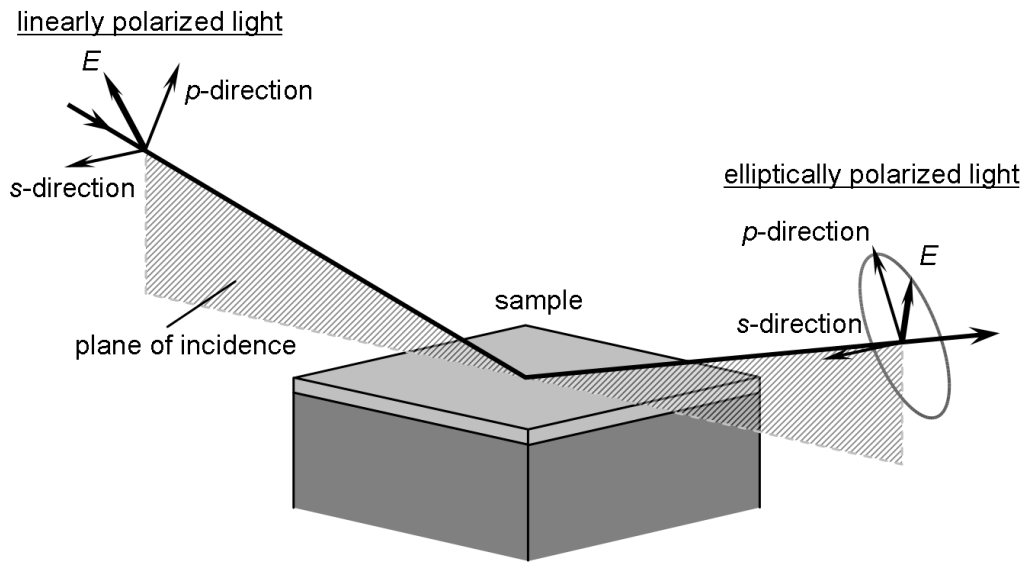

Figure 8.1: Interaction of polarized light with a sample during ellipsometric measurements. Figure reproduced from Brunets [185].

ambient $\mathrm{N}_{2}\left(\mathrm{~N}_{2}\right.$ bubbled through de-ionized water at room temperature). Selected wafers received a post-oxidation anneal (POA) in $\mathrm{N}_{2}$ at $900^{\circ} \mathrm{C}$ for $30 \mathrm{~min}$ prior to the Al metallization.

\subsection{Spectroscopic ellipsometry}

Ellipsometry is a very sensitive non-destructive surface and thin film optical measurement technique that uses polarized light and measures the polarisation state of the light beam reflected from the sample. Ellipsometry can be used to determine thin film thicknesses and thin film optical constants.

During the ellipsometric measurements, an input beam with known linear polarization state is focused onto the sample (as shown in Figure 8.1). The polarization state is described by specifying its components along two orthogonal axes, the so-called p- and s-directions, in the plane perpendicular to the direction of beam propagation. The p-direction is defined as lying in the plane of incidence (which is defined as the plane containing the incident and reflected beams and the vector normal to the sample surface). The s-direction (from the German word Senkrecht) lies perpendicular to the p-direction. The p-direction, s-direction, and direction of propagation define a right-handed Cartesian coordinate system.

The beam becomes elliptically polarized because of the reflection from 
the sample surface. The values for ellipsometric parameters $p s i(\Psi)$ and delta $(\Delta)$ are extracted by measuring the polarization state of the reflected beam. The change in polarization state, $\rho$, is commonly expressed with $\Psi$ and $\Delta$ and defined as

$$
\rho=\tan (\Psi) \mathrm{e}^{\mathrm{i} \Delta}=\frac{\tilde{R}_{p}}{\tilde{R}_{S}} .
$$

These values are related to the ratio of Fresnel reflection coefficients $\tilde{R}_{\mathrm{p}}$ and $\tilde{R}_{\mathrm{s}}$ for $\mathrm{p}$ - and s-polarized light, respectively [171]. In other words, $\tan (\Psi)$ is the magnitude of the reflectivity ratio, and $\Delta$ is the phase.

Psi and delta values are measured as a function of wavelength in Spectroscopic Ellipsometry (SE). This yields much more information about the sample compared to single-wave SE. Therefore, it is possible to analyze the data in specific spectral regions where the required model parameters are more sensitive.

The thickness of the deposited layers was determined by a Woollam M2000U spectroscopic ellipsometer with NIR (near-infrared) option. The highspeed CCD detector collects intensities of over 700 wavelengths simultaneously, covering the spectral range of 245-1700 nm. The M-2000U was used in "ex-situ" mode. Additionally, the ellipsometer was mounted on the process chamber to perform "in-situ" thin film monitoring and process control during PECVD.

The thickness of the film as well as the optical constants can be extracted from model-based analysis of the ellipsometry data. The ellipsometry data can be expressed in terms of the material optical constants $n$ (index of refraction) and $k$ (absorption coefficient), or, equivalently, the pseudo-dielectric function $\langle\varepsilon\rangle$, which is represented by a real $\left\langle\varepsilon_{1}\right\rangle$ and imaginary $\left\langle\varepsilon_{2}\right\rangle$ part. Prior to the film deposition, the pseudo-dielectric function of the substrate was determined. For the H-terminated c-Si substrates, the pseudo-dielectric function was modeled with a semi-infinite silicon substrate. The substrate modeling of the native and thermal oxide substrates required an additional Cauchy layer to describe the contribution of the transparent oxide film to the pseudo-dielectric function of the substrate [171].

\subsection{Electrical characterization}

\subsubsection{Capacitance-voltage $(C-V)$ characteristics}

Very important information can be extracted from the capacitance measurement of MOS structures, such as dielectric capacitance, equivalent (or electrical) film thickness, the silicon doping, interface trap density, fixed charge at 
the $\mathrm{Si}-\mathrm{SiO}_{2}$ interface, and the charge density situated in the dielectric's bulk volume.

The high-frequency capacitance of MOS structures was measured by superimposing a small ac signal $(10 \mathrm{kHz}-1 \mathrm{MHz})$ on a ramped dc bias between the $\mathrm{Al}$ gate and the substrate. The high frequency $C-V$ were carried out using a Hewlett-Packard 4275A multi-frequency meter. The quasi-static $C-V$ curve was measured with a Hewlett-Packard 4140B pA meter, by applying only a dc bias with a sweep rate of $0.1 \mathrm{~V} / \mathrm{s}$. The bias was applied to the metal gate. The measurements started in inversion through depletion to accumulation, and then swept back through depletion to inversion. The measurements started with a light flash and stabilization, which assured that the MOS device had reached the equilibrium inversion condition before the sweep began.

The gate bias applied to a MOS structure can be described [184] by

$$
V_{\mathrm{G}}=V_{\mathrm{FB}}+\Psi_{\mathrm{S}}+V_{\mathrm{ox}}=V_{\mathrm{FB}}+\Psi_{\mathrm{S}}+\frac{-Q}{C_{\mathrm{ox}}},
$$

where $Q$ is the (gate bias induced) substrate charge per unit area. The ratio of substrate charge and oxide capacitance, $C_{\mathrm{Ox}}$, is the potential difference responsible for the substrate charge. The flatband voltage, $V_{\mathrm{FB}}$, and surface potential, $\Psi_{S}$, are gate voltage components, required to balance the intrinsic charge in the semiconductor and the potential drop in the substrate, respectively. To be able to understand the observed shape of the $C-V$ characteristic, the response of the charge inside the structure to the ac-signal has to be considered for low and high probing-frequencies. The concepts of $C-V$ measurements are briefly introduced below. Unless otherwise specified, this material follows Nicollian and Sze $[181,186]$.

The MOS device is a voltage divider with voltage drops across the oxide, and silicon space charge region (2 capacitors in series). The voltage dependent capacitance of the silicon space charge region follows the free carrier response at the space charge-neutral region edge. In accumulation, the dc-state is characterized by a pileup of majority carriers near the oxide-semiconductor interface. In this case, only the majority carriers play a role. This means that the state of the device can change very rapidly (in the order $10^{-10}$ to $10^{-13}$ seconds). Therefore, the device is able to follow the applied ac-signal; a small charge $\partial Q$ will be added or subtracted on the two sides of the oxide. For either high or low probing-frequencies can therefore be concluded that the measured capacitance, $C_{\text {acc }}$, equals the oxide capacitance, $C_{o x}$ :

$$
C_{\text {acc }}=C_{\mathrm{ox}}=\frac{\varepsilon_{\mathrm{ox}} \varepsilon_{0} A_{\text {gate }}}{T_{\mathrm{ox}}},
$$


where $\varepsilon_{\mathrm{Ox}}$ is the relative permittivity of silicon dioxide and $\varepsilon_{0}$ is the permittivity of vacuum; $A_{\text {gate }}$ is the area under the gate (capacitor area) and $T_{o x}$ is the oxide thickness.

The capacitance will not rise back to $C_{\mathrm{ox}}$ in inversion for high probing frequencies, as the silicon space charge capacitance, $C_{S}$, will saturate near its minimum because minority carrier generation cannot keep up with the ac signal. These two capacitances in series give the following result:

$$
C_{\text {inv }}=\frac{C_{\mathrm{ox}} C_{\mathrm{S}}}{C_{\mathrm{ox}}+C_{\mathrm{S}}} .
$$

In depletion, $C_{S}$ can be found from the slope of the $C-V$ curve:

$$
C_{\mathrm{S}}=\frac{-\partial Q}{\partial \Psi_{\mathrm{S}}}
$$

For a low frequency measurement (e.g. quasi-static), $C=C_{\text {ox }}$ in strong accumulation or strong inversion. For strong accumulation, this is due to the small band bending resulting from the large majority density at the interface, i.e. the accumulation charge increases exponentially with band bending so that $C_{S} \gg C_{o x}$. For strong inversion, the inversion charge also increasing exponentially with band bending and the inversion layer screens the space charge region from the surface field so that there is little majority-carrier response to the gate voltage. Therefore, $C_{S} \gg C_{o x}$ in inversion and for a metal gate device,

$$
C_{\text {acc }}=C_{\text {inv }}=C_{\text {ox }} \text {. }
$$

\subsubsection{Effect of oxide fixed charge and interface traps}

Oxide fixed charges are charge states in the oxide that do not change occupancy during the $C-V$ sweep. Accordingly, oxide fixed charge induces a parallel shift in the $C-V$ curve at any applied bias (see Figure 8.2). The oxide charge shifts the flatband voltage $V_{\mathrm{FB}}$ :

$$
V_{\mathrm{FB}}=\varphi_{\mathrm{ms}}-\frac{1}{C_{\mathrm{ox}}} \int_{0}^{T_{\mathrm{ox}}} \frac{x}{T_{\mathrm{ox}}} \rho_{\mathrm{ox}}(x) \mathrm{d} x,
$$

where $\varphi_{\mathrm{ms}}$ is the metal-semiconductor work-function difference, $x$ is the distance from the gate, $T_{\mathrm{Ox}}$ is the thickness of the oxide, and $\rho_{\mathrm{ox}}(x)$ is the oxide charge density in a volume [182]. A determination of the charge distribution and its location in the oxide is needed to solve the integral. For thermally grown oxides, the flatband voltage can be calculated using the standard expression:

$$
V_{\mathrm{FB}}=\varphi_{\mathrm{ms}}-\frac{Q_{\mathrm{f}}}{C_{\mathrm{ox}}}
$$


where $Q_{\mathrm{f}}$ is the fixed charge near the $\mathrm{Si}-\mathrm{SiO}_{2}$ interface [182].

In contrast to fixed charges, interface traps change occupancy with voltage when the trap state is swept through the Fermi level. A trap that is an acceptor state is neutral when empty and negatively charged when occupied by an electron. A trap that is a donor state is neutral when occupied by an electron and positively charged when empty. When free carriers are present in numbers that are not significantly greater than the interface trap density (i.e. weak accumulation or weak inversion), interface traps add a measurable capacitance that is in parallel with the space charge capacitance. Eventually, the effect is a decreased slope of the $C-V$ curves when more interface traps are present (see Figure 8.2). One method to determine the interface trap density $\left(D_{\text {it }}\right)$ using quasi-static and high-frequency capacitance is the Castagné method [187]. The expression for $D_{\text {it }}$ at an applied gate voltage is:

$$
D_{\mathrm{it}}=\frac{1}{e}\left(\frac{1}{1 / C_{\mathrm{LF}}-1 / C_{\mathrm{ox}}}-\frac{1}{1 / C_{\mathrm{HF}}-1 / C_{\mathrm{ox}}}\right),
$$

where $C_{\mathrm{LF}}$ and $C_{\mathrm{HF}}$ are the quasi-static and the high-frequency capacitance at the applied voltage.

\subsubsection{Current-voltage $(I-V)$ characteristics}

Besides good interface properties, the dielectric needs to possess low leakage current, high breakdown field and preferably a conduction mechanism that is not trap-related. All these properties can be extracted from the current-voltage characteristics $(I-V)$ or current density-electric field $(J-E)$ curves.

The measurements were performed with a Hewlett-Packard 4156B parameter analyser. A positive voltage was applied on the gate, thus electrons were injected from the substrate and the silicon substrate was in accumulation. 


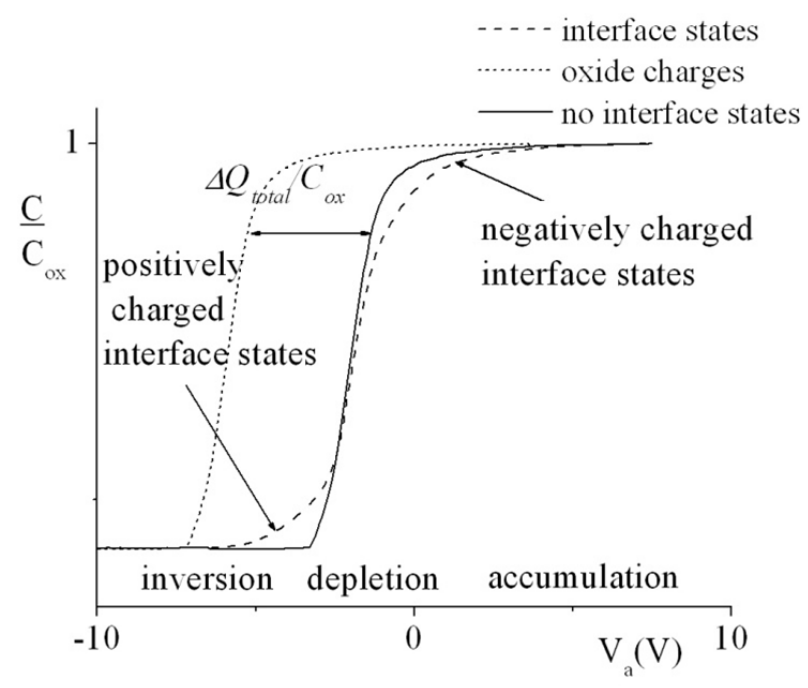

Figure 8.2: $\mathrm{C}-V$ plot demonstrating the effect of oxide charges and interface states. Regions of inversion, depletion and accumulation are assigned to a device without interface states. Reprinted from Bystrova [188]. 


\section{Characterization of the film properties}

The electrical and physical characteristics of the silicon dioxide layers produced in our remote ICPECVD reactor are evaluated. The experimentally found characteristics are compared with the predicted ones in section 6.5. This section summarizes the work published in $[156,189]$.

\subsection{Deposition rate and material density}

\subsubsection{Silane fraction of $0.8 \%$}

Table 9.1 summarizes the deposition rates and the optically measured film thicknesses at several process conditions. This table also contains the electrical film thicknesses and flatband voltages both obtained from $C-V$ measurements (see section 8.4).

The $\mathrm{SiO}_{2}$ deposition rate decreased from $4.7 \mathrm{~nm} / \mathrm{min}$ at $2 \mathrm{~Pa}$ to 3.5 $\mathrm{nm} / \mathrm{min}$ at $6 \mathrm{~Pa}$ (see Table 9.1), whereas one could expect similar deposition rates from the plasma modeling. The SE measurements additionally indicated a lower material density at 1-2 Pa compared to $6 \mathrm{~Pa}$. The measured refractive index $(n)$ at $632 \mathrm{~nm}$ was 1.42 at total pressures of 1 and $2 \mathrm{~Pa}$, whereas at $6 \mathrm{~Pa} n$ increased to its "normal" value of 1.46 for stoichiometric $\mathrm{SiO}_{2}$. The lower material density at 1-2 Pa compared to $6 \mathrm{~Pa}$ was confirmed by $C-V$ measurements. The use of the dielectric constant of $\mathrm{SiO}_{2}$ resulted in the electrical film thickness lower than the measured optical thickness for films deposited at 1 and 2 $\mathrm{Pa}$ (see Table 9.1). Both the electrical $(C-V)$ and optical (SE) methods measure the same thicknesses for films deposited at 6 StateplacePa. The lower density 
at 1-2 Pa could be caused by building an inappropriate chemical network resulting in nano- or micro-voids. This is in agreement with our modeling that indicated that atomic silicon strongly dominates at 1-2 $\mathrm{Pa}$ (Figure 6.3, right). At $6 \mathrm{~Pa}$, however, densities of the other $\mathrm{SiH}_{x}$ radicals increase dramatically and approach the level of atomic Si. The higher material density at $6 \mathrm{~Pa}$ is due to a better surface migration of the $\mathrm{SiH}_{3}$ species and a lower flux of atomic Si towards the film surface.

At a total pressure of $2 \mathrm{~Pa}$, the refractive index increases from 1.42 at $150^{\circ} \mathrm{C}$ to 1.46 at $400^{\circ} \mathrm{C}$, which indicates a stoichiometric $\mathrm{Si} / \mathrm{O}$ ratio and appropriate layer density.

\subsubsection{Silane fraction of $0.08 \%$}

The $\mathrm{SiO}_{2}$ deposition rate remained approximately $0.6 \mathrm{~nm} / \mathrm{min}$ for the entire pressure range 1-6 $\mathrm{Pa}$ at a substrate temperature of $150^{\circ} \mathrm{C}$ (see Table 9.1). A small increase of the deposition rate at 2 and $6 \mathrm{~Pa}$ compared to $1 \mathrm{~Pa}$ still can be seen. The measured $n$ was in this case 1.46 at total pressures of 1 and 2 $\mathrm{Pa}$, whereas at $6 \mathrm{~Pa} n$ decreased to 1.42 . This indicated the stoichiometric $\mathrm{Si} / \mathrm{O}$ ratio of the material and an appropriate density at 1-2 $\mathrm{Pa}$, and a lower material density at a pressure of $6 \mathrm{~Pa}$. Modeling also predicted a highert film density at 1 $\mathrm{Pa}$ (cf. Table 6.2). This was due to the presence of $\mathrm{SiH}_{x}$ radicals at this pressure, whereas atomic silicon was calculated to be the only silicon precursor at $6 \mathrm{~Pa}$.

Again, at $2 \mathrm{~Pa}$, denser layers are deposited at $400^{\circ} \mathrm{C}$ than at $150^{\circ} \mathrm{C}$ (based on the same observations as above).

\subsection{Leakage current}

Leakage currents were extracted from the corresponding $J-E$ curves. The potential drop across the oxide $\left(V_{\mathrm{ox}}\right)$ was calculated by equation (8.2) whereby the flatband voltages $\left(V_{\mathrm{FB}}\right)$ were derived from the corresponding $C-V$ measurements, and surface potentials $\left(\Psi_{S}\right)$ were calculated according to Pierret $[190,191]$. The oxide electric fields $\left(E_{\text {ox }}\right)$ were calculated from $V_{\text {ox }}$ and the measured optical film thicknesses.

\subsubsection{Silane fraction of $0.8 \%$}

Layers deposited at a pressure of 1-2 Pa exhibit a relatively high leakage current (Figure 9.1a). The current density saturates at a low electric field of 2 $\mathrm{MV} / \mathrm{cm}$, which indicates a high concentration of traps. The ledges between 2 and $8 \mathrm{MV} / \mathrm{cm}$, noticeable in Figure 9.1a, are probably related to a conduction 


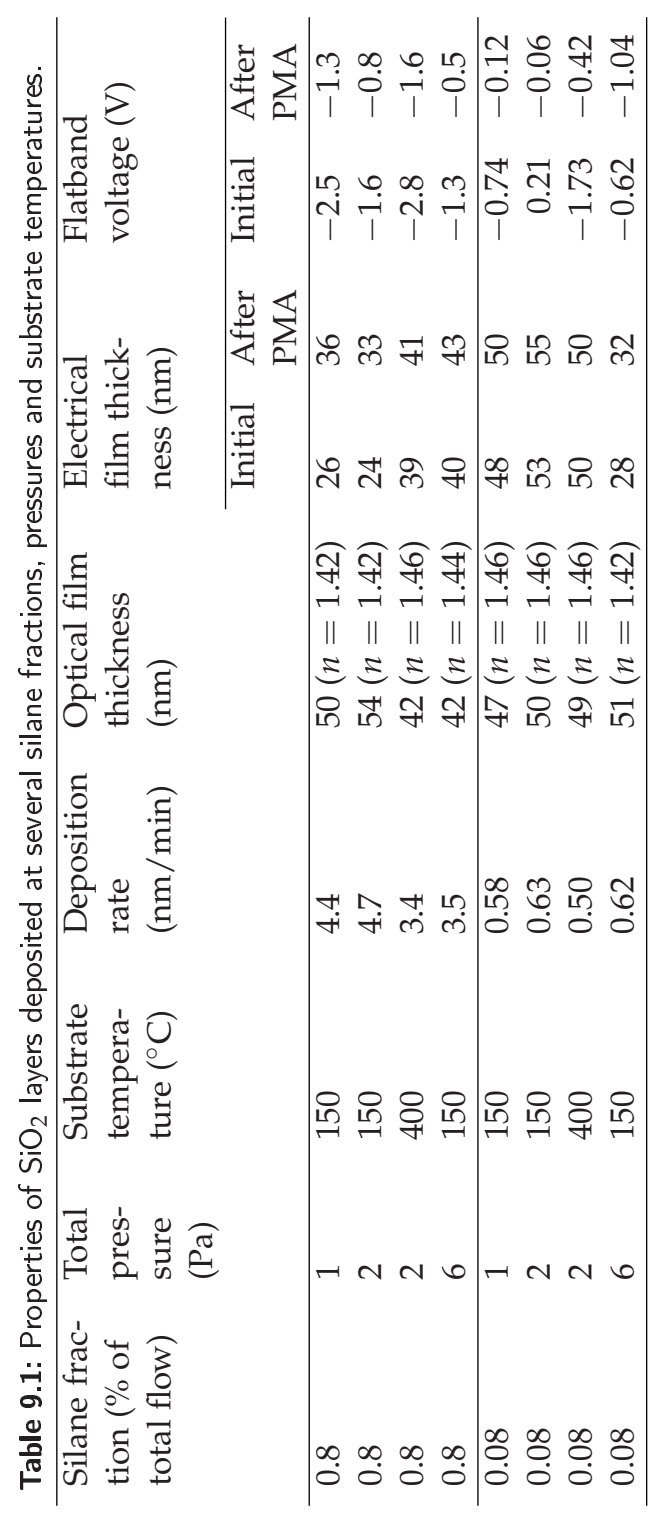


mechanism caused by bulk traps [192]. In other words, electrons are hopping from trap to trap until they reach the electrode. This is caused by the electric field we apply. However, the capacity of a single trap is limited. This means that the current should saturate at certain voltage and we see this effect as the flat part of the $J-E$ curve. In this regime, the current is limited by the transport of electrons to/from each trap, i.e., by the trap capture/escape time, not by the voltage. Such traps can be caused, for example, by extra silicon [193]. However, the refractive index of 1.42 (see Table 9.1) indicated a lower material density than the formation of such silicon-rich layers, since extra silicon should increase the refractive inde ${ }^{1}$. Therefore, traps are more likely to be caused by a lower material density because of building inappropriate chemical network (e.g., dangling bonds partially or fully satisfied with hydrogen instead of Si-O bonds). The leakage current decreases approximately two orders of magnitude when the pressure during deposition is increased to $6 \mathrm{~Pa}$. Please note that traps responsible for this conduction mechanism are less affected by the PMA (see Figure 9.1a).

The higher leakage current at $1 \mathrm{~Pa}$ compared to $6 \mathrm{~Pa}$ was predicted correctly by the modeling ( $c f$. Table 6.2). This prediction was based on two observations: (i) the total absence of $\mathrm{SiH}_{x}$ precursors in plasma at $1 \mathrm{~Pa}$, and (ii) the higher density of atomic hydrogen at $1 \mathrm{~Pa}$. Regarding (i), electrically better films were expected at $6 \mathrm{~Pa}$ due to a better surface migration of the other $\mathrm{SiH}_{x}$ species and a lower flux of atomic Si towards the film surface. Regarding (ii), one could expect that the higher hydrogen density at $1 \mathrm{~Pa}$ will enhance hydrogen abstraction, and hence will create films with more traps and therefore higher leakage currents.

\subsubsection{Silane fraction of $0.08 \%$}

Unlike the higher $\mathrm{SiH}_{4}$ fraction, the layers deposited at a pressure of $6 \mathrm{~Pa}$ exhibit a high leakage current (9.1b). The leakage current drops within approximately two orders of magnitude with decreasing the pressure to $2 \mathrm{~Pa}$, and another 2 orders of magnitude when the pressure is further decreased to $1 \mathrm{~Pa}$.

Again, the predictions by modeling (i.e., higher leakage currents at $6 \mathrm{~Pa}$ than at $1 \mathrm{~Pa}$ ) were in accordance with these results (cf. Table 6.2). The best film quality was expected at a pressure of $1 \mathrm{~Pa}$ due to the presence of small portions of the other $\mathrm{SiH}_{x}$ radicals. Furthermore, the density of atomic hydrogen is much lower at $1 \mathrm{~Pa}$ than at $6 \mathrm{~Pa}$ (see Figure 6.3). Again, the higher density at $6 \mathrm{~Pa}$

1. Adding for example 0.7 volume\% of extra silicon to a $\mathrm{SiO}_{2}$ layer increases $\mathrm{n}$ to 1.47 (at 632.8 $\mathrm{nm}$ ). Calculated (Bruggeman EMA approximation of a-Si and $\mathrm{SiO}_{2}$ ) using CompleteEASE code, version 3.60 by J.A. Woollam Co., Inc. 

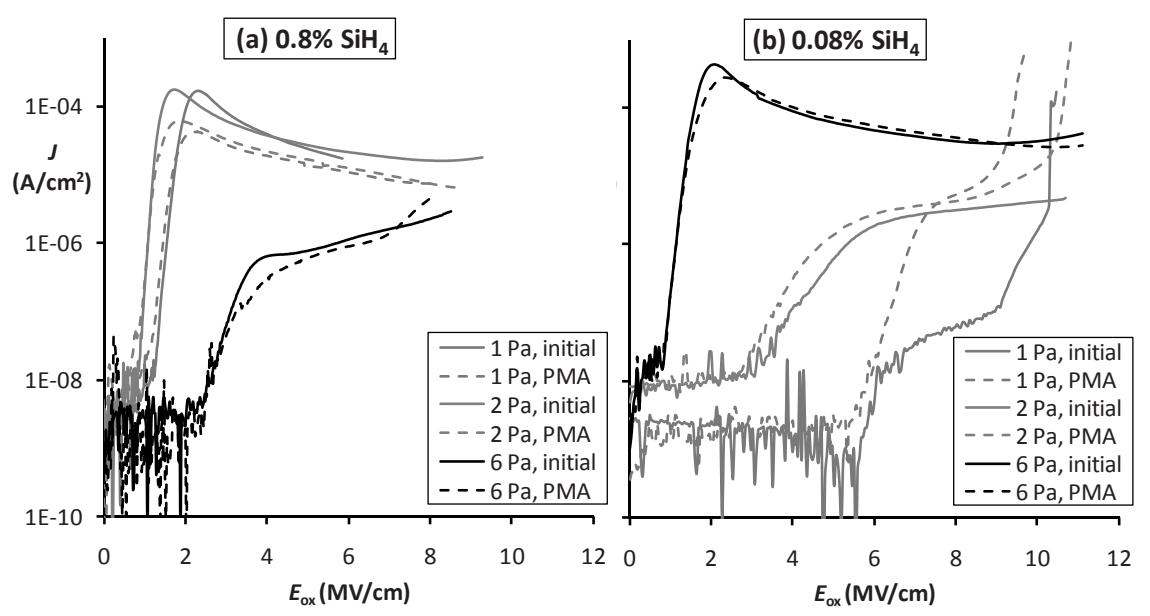

Figure 9.1: $J-E$ characteristics of silicon dioxide layers ( $\sim 50 \mathrm{~nm}$ thick).

might cause this might enhance hydrogen abstraction, and hence will create films with more traps and therefore higher leakage currents.

The J-E characteristic of the film deposited at $2 \mathrm{~Pa}$ and a substrate temperature of $400^{\circ} \mathrm{C}$ is not shown in Figure 9.1 because of clarity. As denser layers should be grown at higher temperatures due to enhanced surface migration and better hydrogen desorption, the $2 \mathrm{~Pa} / 400^{\circ} \mathrm{C}$-layers are expected to show lower leakage currents than the $2 \mathrm{~Pa} / 150^{\circ} \mathrm{C}$-layers. By comparing the 2 $\mathrm{Pa} / 150^{\circ} \mathrm{C}$-curve from Figure $9.1 \mathrm{~b}$ with the $2 \mathrm{~Pa} / 400^{\circ} \mathrm{C}$-curve from Figure 9.2, one can see that the film deposited at $400^{\circ} \mathrm{C}$ indeed shows lower leakage currents. The $J-E$ characteristics of the film deposited at $1 \mathrm{~Pa}$ and $150^{\circ} \mathrm{C}$ and the thermally grown oxide film are also shown in Figure 9.2. Both PECVD films exhibit very low leakage currents at the electric field strength of $6.5 \mathrm{MV} / \mathrm{cm}$, which is comparable to the leakage currents of thermally grown oxides. However, the $J-E$ characteristic of the film deposited at $1 \mathrm{~Pa}$ and $150^{\circ} \mathrm{C}$ exhibits remnants of the ledges (at 7-8 MV/cm) that are probably still related to a conduction mechanism caused by bulk traps. 


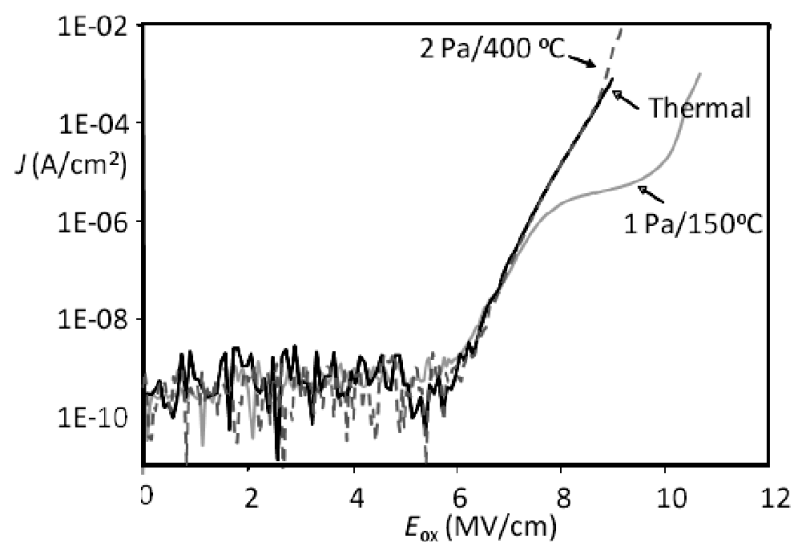

Figure 9.2: $J-E$ characteristics of the thermally grown oxide film and the films deposited at $1 \mathrm{~Pa} / 150{ }^{\circ} \mathrm{C}$ and $2 \mathrm{~Pa} / 400^{\circ} \mathrm{C}$ (all after PMA).

\subsection{Capacitance-voltage characteristics}

\subsubsection{Silane fraction of $0.8 \%$}

$C-V$ measurements of several silicon dioxide layers are shown in Figure 9.3. Figure 9.3a reveals a strong influence of total pressure on $C-V$ curves. Table 9.1 compares the oxide thickness calculated using the dielectric constant of stoichiometric $\mathrm{SiO}_{2}$ and the optical film thickness of the SE measurements. The use of the dielectric constant of $\mathrm{SiO}_{2}$ resulted in the electrical film thickness lower than the measured optical thickness for films deposited at 1 and $2 \mathrm{~Pa}$. Both the electrical $(C-V)$ and optical (SE) methods measure the same thicknesses for films deposited at $6 \mathrm{~Pa}$. Furthermore, Figure 9.3a shows that the PMA drastically decreases the oxide capacitance for the film deposited at $1 \mathrm{~Pa}$. Incorporation of water in these films could explain the low electrical thickness at $1-2 \mathrm{~Pa}$ before the PMA. The capacitance is $\sim 46 \%$ higher before the PMA; we can calculate that this accounts for approx. $2 \%$ of water in the layers (roughly approximated by: $\varepsilon_{\text {effective }}=x \cdot \varepsilon_{\mathrm{H}_{2} \mathrm{O}}+(1-x) \cdot \varepsilon_{\mathrm{SiO}_{2}}$, with $\varepsilon_{\text {effective }}$ as the effective dielectric constant of the oxide, $x$ as the volume fraction of water and $\varepsilon_{\mathrm{SiO}_{2}}=3.9$ and $\varepsilon_{\mathrm{H}_{2} \mathrm{O}}=80$ ). The presence of water can partly explain the somewhat low refractive index of 1.42 (since $n_{\mathrm{H}_{2} \mathrm{O}}=1.33$ ). During PMA, water is desorbed from these films (or reduced on the Al-surface) thereby reducing the oxide capacitance (see Figure 9.3a). However, Table 9.1 shows that also after PMA, the electrically obtained oxide thickness is still smaller than the optical 


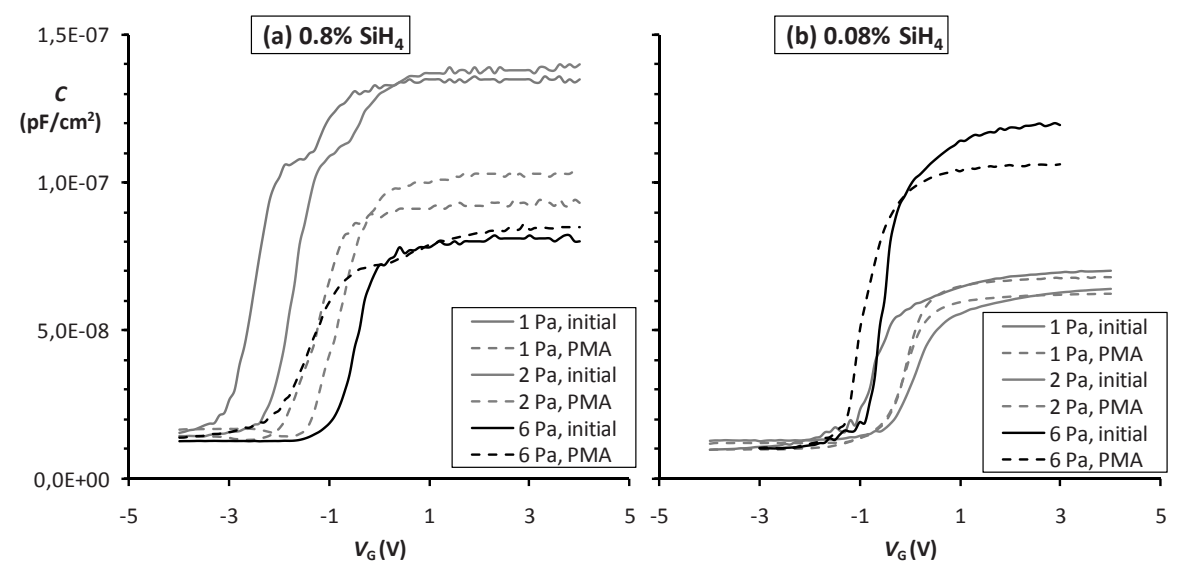

Figure 9.3: $C-V$ characteristics of silicon dioxide layers ( $\sim 50 \mathrm{~nm}$ thick).

thickness for films deposited at 1 or $2 \mathrm{~Pa}$. This indicates that the dielectric characteristics of these films do not equal those of thermally grown oxide ( $c f$. Table $6.2)$.

$\mathrm{C}-V$ measurements of the layers deposited at $2 \mathrm{~Pa}$ and $400^{\circ} \mathrm{C}$ showed however an excellent agreement of physical and electrical film thickness (see Table 9.1). This should point to the importance of surface reactions, surface migration and hydrogen desorption, enhanced at $400^{\circ} \mathrm{C}$, which resulted in a better dielectric quality. Nevertheless, the same dielectric quality was measured for layers deposited at $150^{\circ} \mathrm{C}$ and $6 \mathrm{~Pa}$, which can be attributed to an increased fraction of $\mathrm{SiH}_{3}$ radicals in the plasma, as well as to the lower hydrogen density at $6 \mathrm{~Pa}$ (see Figure 6.3, right).

The $C-V$ curves shown in Figure 9.3a also reveal a strong influence of total pressure on fixed oxide charge. The measured flatband voltage $\left(V_{\mathrm{FB}}\right)$ is about $-2.5 \mathrm{~V}$ for the film obtained at a deposition pressure of $1 \mathrm{~Pa}$ (without PMA). Using a value of $-0.2 \mathrm{~V}$ for the metal-semiconductor work-function difference $\left(\phi_{\mathrm{ms}}\right)$ between the $\mathrm{Al}$ contact and $n$-type Si substrate with a doping level of $1.5 \cdot 10^{15} \mathrm{~cm}^{-3}$ [194], one can calculate a positive oxide charge of $2 \cdot 10^{12} \mathrm{~cm}^{-2}$ from equation (8.8). The $V_{\mathrm{FB}}$ shifts to $-1.3 \mathrm{~V}$ with increasing the total pressure from 1 to $6 \mathrm{~Pa}$, which corresponds to a charge of $6 \cdot 10^{11} \mathrm{~cm}^{-2}$. Concluding, we observe a higher fixed charge at 1-2 Pa compared to $6 \mathrm{~Pa}$. This is probably due to enhanced hydrogen abstraction at 1-2 Pa compared to $6 \mathrm{~Pa}$. Abstraction desorbs hydrogen from the film surface thereby leaving 
Si-dangling bonds that were originally passivated by hydrogen. However, also other processes can create a higher fixed charge in the films. Charges can be caused by bombardment (likely by heavy $\mathrm{Ar}^{+}$ions) [195], and, probably, due to implantation of $\mathrm{H}^{+}$atoms (they can penetrate deeply and have less chance to be neutralized) [196]. At low pressures, the density of Ar ions is lower but their bohm velocities ( $c f$. equation (3.9)) are much higher. Therefore, they can probably create damage in the film (during the entire growth) and on the substrate (during nucleation) and create somehow a positive charge. At $6 \mathrm{~Pa}$, the Ar ion density increases but their bohm velocities significantly decrease, which means less severe bombardment.

In Table 9.1 one can see that $V_{\mathrm{FB}}$ of the layers deposited at $400^{\circ} \mathrm{C}$ and $2 \mathrm{~Pa}$ is more negative than that of the layers deposited at $150^{\circ} \mathrm{C}$ and the same pressure. It indicates a higher positive charge is incorporated in the layers at elevated temperatures. At the same time, the deposition rate measured by ellipsometer decreases from 4.7 to $3.4 \mathrm{~nm} / \mathrm{min}$ when the temperature is increased (see Table 9.1). As mentioned earlier, the refractive index also increases from 1.42 at $150^{\circ} \mathrm{C}$ to 1.46 at $400^{\circ} \mathrm{C}$, which indicates a stoichiometric $\mathrm{Si} / \mathrm{O}$ ratio and appropriate layer density.These two results lead to the conclusion that denser layers were deposited at $400^{\circ} \mathrm{C}$. Since the temperature of the wafer does not influence the supply of reactive species from the plasma, this densification is probably caused by the enhanced surface migration of the adsorbed species at elevated temperatures and enhanced hydrogen desorption. Hydrogen abstraction is probably also enhanced at $400^{\circ} \mathrm{C}$. This leaves a film that contains more Si-dangling bonds, and hence a higher positive charge.

The PMA significantly decreases the fixed oxide charge, as is well known from literature (see section 6.5 for references). Furthermore, the slope of the $C-$ $V$ curves increases when PMA is applied, which indicates less interface traps after PMA (as explained in section 8.4.2).

\subsubsection{Silane fraction of $0.08 \%$}

One can again notice a strong influence of total pressure on the $C-V$ curves, see Figure 9.3b. The effect of the pressure was, however, opposite compared to that at the higher silane fraction (compare with Figure 9.3a). Table 9.1 shows that the films deposited at total pressures of 1 and 2 Pa have an electrical film thickness which corresponds well to the optical thickness. Also hardly any difference in film thickness is observed after PMA. The film deposited at 6 Pa however showed a large difference in optical and electrical film thickness (51 and $28 \mathrm{~nm}$, resp.). After PMA the electrical thickness slightly increased, which could point to water desorption during PMA. 
The films deposited at $400^{\circ} \mathrm{C}$ show qualitatively similar behavior as the ones deposited with higher silane fraction of $0.8 \%$ (and otherwise the same deposition conditions). Shortly, good agreement between optical and electrical film thickness was observed, which indicates the deposition of dense and stoichiometric $\mathrm{SiO}_{2}$ films. Furthermore, a negative $V_{\mathrm{FB}}$ of $-1.73 \mathrm{~V}$ indicates a larger oxide charge density before PMA than for the films deposited at $150^{\circ} \mathrm{C}$. This higher charge density might be caused by enhanced hydrogen abstraction at $400^{\circ} \mathrm{C}$. This leaves a film that contains more Si-dangling bonds, and hence a higher positive charge.

It can be concluded that at a deposition temperature of $150^{\circ} \mathrm{C}$, the lowest leakage currents are obtained with films deposited at a pressure of $1 \mathrm{~Pa}$, and a silane fraction of $0.08 \%$ (see Figure 9.1). These oxides also show good $C-V$ curves with respect to the oxide's dielectric constant, and fixed charge. Consequently, these films are the most promising ones to be deployed as dielectric in low-temperature-fabricated electronic devices. This will be assessed in section 9.5. However, before we do so, their dielectric behavior will first be compared with thermally grown oxide.

The $C-V$ measurements of the thermally grown oxide film and those of the oxide film deposited at $1 \mathrm{~Pa}$ are compared in Figure 9.4. One can notice that, in the depletion region, the slope of the thermal oxide film is steeper than that of the 1-Pa film. This decreased slope indicates more interface traps (see section 8.4.2). As explained earlier, annealing of aluminum-gate devices in $\mathrm{H}_{2} \mathrm{O}$-containing ambient can provide hydrogen atoms for the trap passivation. Indeed, Figure 9.3b shows that the slope of the $C-V$ curves increases when PMA is applied to the 6-Pa film, which indicates less interface traps after PMA. However, Figure 9.4 indicates that the PMA does not passivate interface traps of the 1-Pa film entirely to the level of the thermally grown oxide. The PMA however significantly decreases the fixed oxide charge in the 1-Pa film (see Figure 9.3b and Table 4.6) as expected from literature. A very low midgap- $D_{\text {it }}$ of $3 \cdot 10^{10} \mathrm{eV}^{-1} \mathrm{~cm}^{-2}$ was obtained from the QS and HF measurements presented in Figure 9.5.

As a last characterization step, the etch rate of our $1 \mathrm{~Pa} / 150^{\circ} \mathrm{C}$-oxides in a $5: 1 \mathrm{BHF}$ solution (5 volume parts of 40\% NH4F mixed with 1 volume part of $49 \% \mathrm{HF}$ ) was measured. The etch rate of our $1 \mathrm{~Pa} / 150^{\circ} \mathrm{C}$-film is 95 $\mathrm{nm} / \mathrm{min}$; this value is close to that of thermally grown oxide $(80 \mathrm{~nm} / \mathrm{min})$ which confirms that this film not only electrically but also chemically resembles thermally grown oxide. 


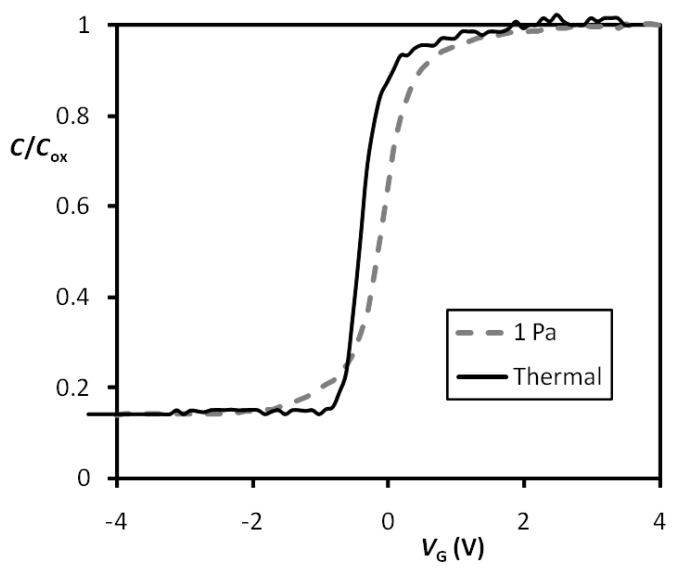

Figure 9.4: $C-V$ curves of the thermal grown oxide film and the film deposited at 1 $\mathrm{Pa}$ and $150^{\circ} \mathrm{C}$ (both after PMA).

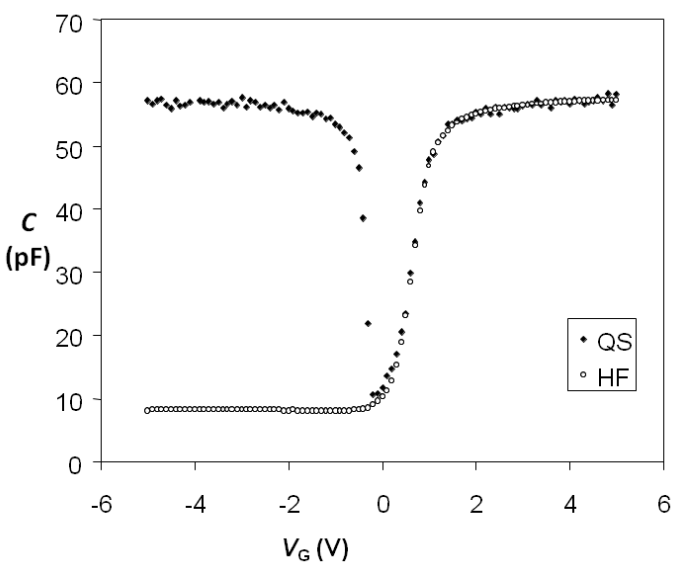

Figure 9.5: $\mathrm{C}-V$ curves of a $35 \mathrm{~nm} \mathrm{PECVD} \mathrm{SiO}_{2}$ layer deposited with an initial silane fraction of $0.08 \%$ at $1 \mathrm{~Pa}$ and $150^{\circ} \mathrm{C}$. 


\subsection{Summarizing the correlations between predicted and measured film properties}

The results were consistent with predictions of the chemical modeling. To summarize, the following effects were explained correctly by the modeling.

First, for a $\mathrm{SiH}_{4}$ fraction of $0.8 \%$ was observed:

1. Lower $\mathrm{SiO}_{2}$ density and more traps in the films deposited at a pressure of $1 \mathrm{~Pa}$ : this resulted in higher leakage currents;

2. close-to-normal $\mathrm{SiO}_{2}$ density and less traps in the films deposited at a pressure of $6 \mathrm{~Pa}$ : this led to the lower leakage current.

Second, for a $\mathrm{SiH}_{4}$ fraction of $0.08 \%$ :

1. close-to-normal $\mathrm{SiO}_{2}$ density and less traps in the films deposited at a pressure of $1 \mathrm{~Pa}$ : this led to the lower leakage current;

2. Lower $\mathrm{SiO}_{2}$ density and more traps in the films deposited at a pressure of $6 \mathrm{~Pa}$ : this resulted in the higher leakage current.

\subsection{Utilization in electronic devices}

Thin film transistors (TFTs) were fabricated in order to confirm the quality of our $0.08 \% \mathrm{SiH}_{4}-1 \mathrm{~Pa}-150^{\circ} \mathrm{C}$ silicon-dioxides. This oxide was used as the gate dielectric. This section summarizes the work published in $[197,198]$.

$100 \mathrm{~mm}, 5-10 \Omega \cdot \mathrm{cm}$ silicon wafers were thermally oxidized to grow a $0.9 \mu \mathrm{m} \mathrm{SiO}{ }_{2}$ layer. Subsequently, a 50 -nm thick a-Si film was deposited by LPCVD (from $\mathrm{SiH}_{4}$ ) at $550^{\circ} \mathrm{C}$, in which lines $\sim 0.5 \mu \mathrm{m}$ wide at a 4 micrometer distance were patterned. A second 100-nm a-Si layer was deposited with the same LPCVD process, resulting in an amorphous film with a periodically varied thickness (see Figure 9.6, left). This morphology was done to control the location of grain boundaries (i.e., create boundaries mostly parallel to this texturing) during laser annealing [198]. The crystallization process is illustrated in Figure 9.6a-c. During the laser crystallization (a), the periodically varied thickness locally results in non-molten lines deeply embedded in the molten silicon (b). These solid regions influence the temperature gradient in the lateral direction perpendicular to the laser scan direction and serve as the crystallization centres for super lateral crystal growth (c). Experimental proof of this concept was given in [198].

The transistors were orientated such that the amount of grain boundaries was minimized, i.e., parallel to the texture [198]. $\mathrm{BF}_{2}^{+}\left(1 \times 10^{15} \mathrm{~cm}^{-2}\right)$ and $\mathrm{As}^{+}$ $\left(4 \times 10^{15} \mathrm{~cm}^{-2}\right)$ ions were implanted at $55 \mathrm{keV}$ to form $\mathrm{p}$ - and n-type regions for 

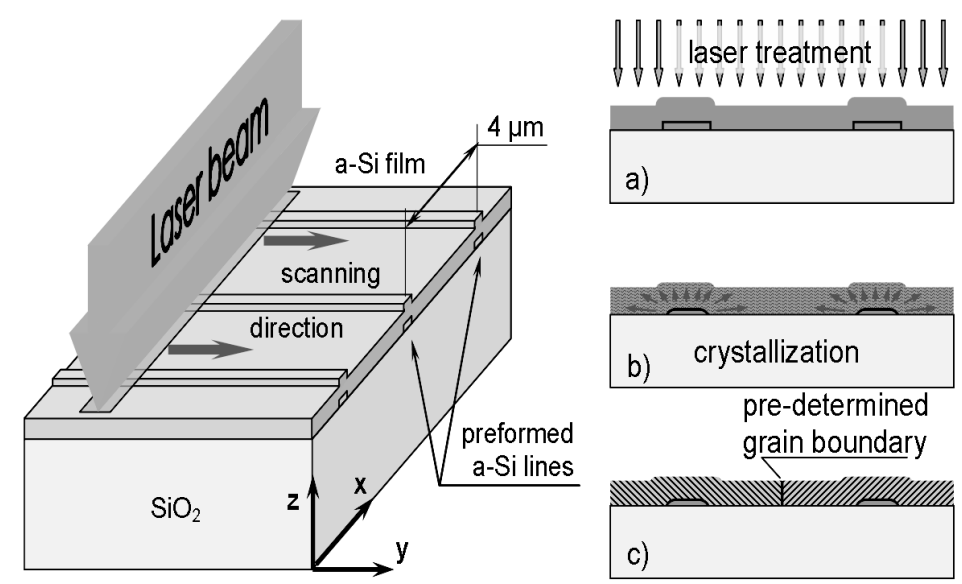

Figure 9.6: Crystallization process of a silicon film with preformed lines. Left: illustration of the crystallization step. Right: (a) laser treatment, melting the silicon film, (b) crystallization process - super lateral growth, (c) finally crystallized film with predefined grain boundaries.

the source and drain. Green-laser annealing was employed for crystallization and dopant activation.

A 100-nm thick $\mathrm{SiO}_{2}$ gate dielectric layer was deposited by means of ICPECVD in $\mathrm{Ar}-\mathrm{N}_{2} \mathrm{O}-\mathrm{SiH}_{4}$ plasma at $150^{\circ} \mathrm{C}$ and pressure $1 \mathrm{~Pa}$. The gas phase contained $0.08 \%$ of $\mathrm{SiH}_{4}$ and $18 \%$ of $\mathrm{N}_{2} \mathrm{O}$. This layer was chosen for its sound dielectric quality, see Figure 9.1, Figure 9.4, and Figure 9.5 in section 9. Finally, contact openings in the $\mathrm{SiO}_{2}$ were etched in a buffered hydrofluoric acid (BHF) solution. The front and back sides were metalized by sputtering a $1 \mu \mathrm{m}$ thick $\mathrm{Al}$ layer, patterned at the front side (defined by photolithography and wet etched) - providing metal contacts to the source/drain regions and forming the gate electrode. The PMA was done in $\mathrm{H}_{2} \mathrm{O} / \mathrm{N}_{2}$ ambient for 10 minutes at $400^{\circ} \mathrm{C}$ (see section 8.2).

The field effect mobility was calculated from the transconductance, $g_{\mathrm{m}}$, in the linear region at low source-drain voltage $\left(V_{\mathrm{DS}}\right)$ as described in [184], see Figure 9.7. The measurements were done by varying the Al-front gate voltage with the Si-bottom gate (i.e. the substrate) grounded. The device channels were undoped for both p- and n-channel TFTs. Figure 9.7 shows typical transfer characteristics obtained at a drain voltage of $-0.1 \mathrm{~V}$ for p-channel TFTs and $0.1 \mathrm{~V}$ for n-channel TFTs. The output characteristics (Figure 9.8) exhibit typi- 


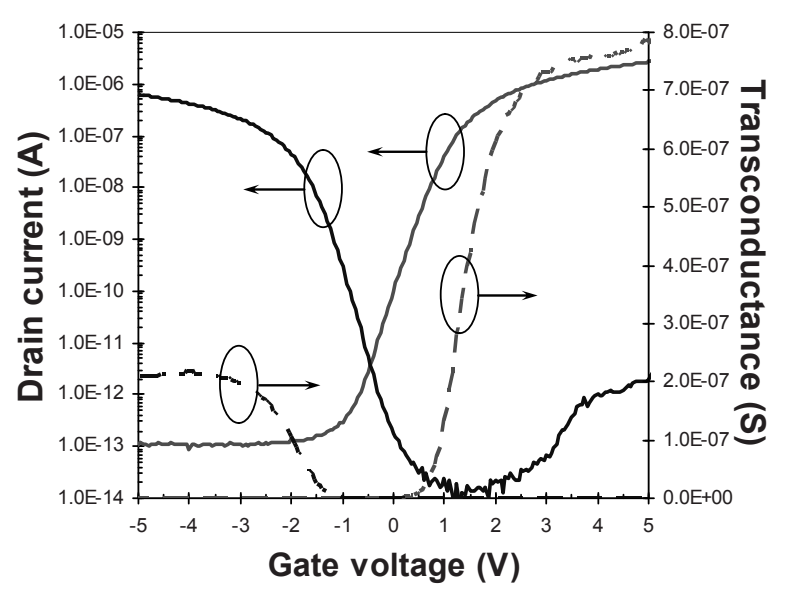

Figure 9.7: Transfer characteristics measured for $n$-channel and $p$-channel TFTs $(W / L=10 \mu \mathrm{m} / 20 \mu \mathrm{m})$ at a drain voltage of $0.1 \mathrm{~V}$ or $-0.1 \mathrm{~V}$. Data taken from [198].

cal linear-saturation curves for the drain current at various gate voltages. This demonstrates the dielectric strength of the gate oxide. This was confirmed by the low leakage current in Figure 9.7. The TFTs show competitive mobilities for both N- and P-MOS transistors, as listed in Table 9.2.

The mobility of the present TFTs using ICPECVD $\mathrm{SiO}_{2}$ is significantly higher than similar TFTs fabricated with ALD $\mathrm{Al}_{2} \mathrm{O}_{3}$ [199]. This mobility enhancement was attributed to the use of a thick high-quality $\mathrm{SiO}_{2}$ gate dielectric in contrast to the earlier used thin $\mathrm{Al}_{2} \mathrm{O}_{3}$ [199]. $\mathrm{Al}_{2} \mathrm{O}_{3}$ has also shown to yield lower mobilities in the case of TFTs on monocrystalline $\mathrm{Si}$ [200]. A comparison with thermal oxide is not straightforward in this case. First, thermal oxide is

Table 9.2: Performance of TFTs with $\mathrm{SiO}_{2}$ gate oxide.

\begin{tabular}{lrr}
\hline Symbol & N-MOS TFT & P-MOS TFT \\
\hline TFT channel sizes $(\mathrm{W} / \mathrm{L})$ & $10 / 20$ & $10 / 20$ \\
Threshold voltage, $V_{\mathrm{T}}(\mathrm{V})$ & 1.5 & -2.0 \\
On-current, $I_{\text {on }}(\mu \mathrm{A})$ & 35.1 & 7.6 \\
Typical $I_{\text {on }}$ full range variation $(\%)$ & 8.2 & 7.5 \\
Subthreshold slope, $S(\mathrm{mV} / \mathrm{dec})$ & 308 & 275 \\
Field effect mobility, $\mu \mathrm{FE}\left(\mathrm{cm}^{2} / \mathrm{Vs}\right)$ & 405 & 128 \\
\hline
\end{tabular}




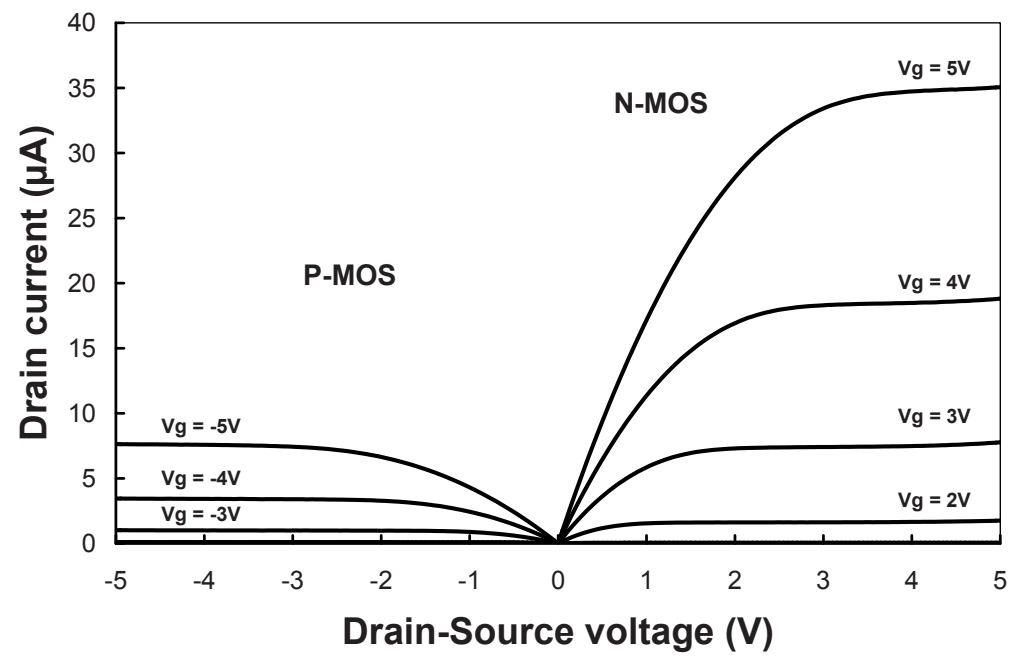

Figure 9.8: Output characteristics measured for $n$-channel and $p$-channel TFTs $(W / L=10 \mu \mathrm{m} / 20 \mu \mathrm{m})$ at different gate voltages. Data taken from [198].

not applicable to low-temperature TFT technology, and second, the quality of thermal oxide on polysilicon is not obvious at all [201-203].

\subsection{Conclusions}

Silicon dioxide films were deposited by means of remote inductively coupled plasma enhanced chemical vapor deposition (ICPECVD), from $\mathrm{SiH}_{4}$ and $\mathrm{N}_{2} \mathrm{O}$ precursors in Ar. Total gas pressure ranged between 1 and $6 \mathrm{~Pa}$ (7.5-45 mTorr), and the substrate temperature was either 150 or $400^{\circ} \mathrm{C}$. The gas phase contained 0.08 or 0.8 vol. $\%$ of $\mathrm{SiH}_{4}$ and $18 \mathrm{vol} \%$ of $\mathrm{N}_{2} \mathrm{O}$, and $\mathrm{rf}(13.56 \mathrm{MHz})$ source power was $300 \mathrm{~W}$. The electrical and physical characteristics of the silicon dioxide layers were evaluated.

At a silane fraction of $\mathbf{0 . 8 \%}$, there were two regimes of plasma operation. The first regime was achieved at relatively low pressures ( 1 and $2 \mathrm{~Pa}$ ) and resulted in the formation of oxide films with a lower density, a high concentration of charge trapping centers, and a significant amount of positive oxide charge. The second regime was realized at a higher pressure of $6 \mathrm{~Pa}$, and led to the deposition of close-to-stoichiometric silicon oxide films having a much 
lower concentration of charge traps and less positive oxide charge. The lowpressure oxides were further characterized by high-leakage current, while the high-pressure oxides had a much lower leakage current.

At a silane fraction of $\mathbf{0 . 0 8 \%}$, again, two regimes of plasma operation were observed. Films with the best dielectric characteristics were achieved at a total pressure of $1 \mathrm{~Pa}$. These films exhibited very low leakage currents at an electric field strength of $6.5 \mathrm{MV} / \mathrm{cm}$, comparable to the leakage currents known for thermally grown oxides. These films films exhibited a very low midgap- $D_{\text {it }}$ of $3 \cdot 10^{10} \mathrm{eV}^{-1} \mathrm{~cm}^{-2}$. The etch rate in BHF was comparable to that of thermally grown oxide. A second regime was realized at higher pressures (2-6 Pa), and resulted in the formation of oxide films with a higher density of electron traps and higher leakage currents.

The existence of the regimes and their influence on the film properties were predicted by chemical modeling. The electrical measurements, plasma characterization and modeling of chemical reactions in $\mathrm{Ar}-\mathrm{N}_{2} \mathrm{O}-\mathrm{SiH}_{4}$ plasmas are demonstrated to be valuable tools the in process control and optimization of ICP reactors.

The best quality oxides (realized at $0.08 \% \mathrm{SiH}_{4}, 1 \mathrm{~Pa}, 150^{\circ} \mathrm{C}$ ) were used as the gate dielectric in $\mathrm{p}$ - and n-channel poly-Si TFTs. This resulted in highperformance TFTs. 
Net negative charge in $\mathrm{SiO}_{2}$ dielectric layers

\subsection{Introduction}

The quality of our best oxides (realized at 0.08 vol. $\% \mathrm{SiH}_{4}, 1 \mathrm{~Pa}, 150^{\circ} \mathrm{C}$ ) was partially characterized in section 9 and 9.5 in the preceding chapter. In brief, these layers exhibited excellent $I-V$ characteristics and low interface state densities $\left(D_{\text {it }}\right)$. These oxides were applied as gate dielectrics in low-temperature TFTs and demonstrated competitive mobility values and low off-currents. However, an important characteristic of these layers has not been discussed yet. In contrast to thermally grown silicon dioxide, in our ICP-oxide the net charge appeared to be negative and a function of layer thickness. We devoted a separate chapter to this effect because of its novelty and the expected impact on the threshold voltage of field-effect devices affecting their performance. This fixed charge may be exploited in particular applications, e.g. the reduction of surface recombination losses in photovoltaic devices by electrostatically shielding the minority charge carriers using internal electric fields [204].

We demonstrate in this section that two mechanisms can contribute to the film growth and the formation of charges, namely plasma oxidation of the silicon substrate and chemical vapor deposition. We suggest that plasma oxidation mainly results in the growth of the first nm-range of oxide thickness, thereby offering a possible mechanism for the negative charge formation. This work has been published in [205]. 


\subsection{Results and discussion}

Silicon dioxide films were grown by means of remote inductively coupled plasma enhanced chemical vapor deposition (ICPECVD) in $\mathrm{Ar}-\mathrm{N}_{2} \mathrm{O}-\mathrm{SiH}_{4}$ plasmas at $150^{\circ} \mathrm{C}$ and at a total pressure of $1 \mathrm{~Pa}$ as described in chapter 8 . The gas phase contained 0.08 vol. $\%$ of $\mathrm{SiH}_{4}$ and 18 vol. $\%$ of $\mathrm{N}_{2} \mathrm{O}$.

In Figure 10.1, in situ SE measurements are presented from two experiments. The first experiment (open circles) represents the first few minutes of a typical deposition process in $\mathrm{Ar}-\mathrm{N}_{2} \mathrm{O}-\mathrm{SiH}_{4}$ plasma. Whereas the second experiment (solid triangles) was carried out without $\mathrm{SiH}_{4}$ in the gas phase, i.e., in $\mathrm{Ar}-\mathrm{N}_{2} \mathrm{O}$ plasma. Clearly, oxide growth was also observed without $\mathrm{SiH}_{4}$, i.e., by plasma oxidation (solid triangles). This leads to the conclusion that (at these process conditions) the formation of silicon dioxide films is due to two mechanisms: oxidation and CVD. The inset of Figure 10.1 shows the calculated growth rates of the oxidation and CVD components. Figure 10.1 shows a non-linear fast initial oxide growth regime that gradually transfers into a linear regime with a constant deposition rate of $0.6 \mathrm{~nm} / \mathrm{min}$ (cf. Table 9.1). One can conclude that initial oxide formation is due to oxidation of Si. Plasma oxidation dominates for the first 2.5 nanometers of oxide growth, followed by mainly CVD for the thicker layers.

Layer properties are probably different for layers formed by two different mechanisms. This difference can be observed particularly for very thin layers when thicknesses of the two differently formed sub-layers are comparable. Instead of a positive charge, as normally is observed in $\mathrm{SiO}_{2}$ films, a negative oxide charge of $5 \cdot 10^{11} \mathrm{~cm}^{-2}$ can be calculated from the high-frequency $C-V$ curve (see Figure 9.5).

This oxide charge was studied in more detail as a function of layer thickness. The extracted value of the oxide charge is largely dependent on the absolute value of $\varphi_{\mathrm{ms}}$ (see equations (8.7),(8.8)). There is however variation in published $\varphi_{\mathrm{ms}}$ values. As an example, for Al on n-type Si with a doping level of $1.5 \cdot 10^{1} 5 \mathrm{~cm}^{-3}$, Sze [181] published a value of $-0.2 \mathrm{~V}$ while Pierret [206] reported $-0.3 \mathrm{~V}$. To determine $\varphi_{\mathrm{ms}}$ for our system, $\varphi_{\mathrm{ms}}$ was measured on thermally grown oxide (dry oxidation at $950^{\circ} \mathrm{C}$ followed by 20 -min. POA). Rewriting equation (8.8) one gets

$$
V_{\mathrm{FB}}=\varphi_{\mathrm{ms}}-\frac{Q_{\mathrm{f}} T_{\mathrm{ox}}}{K_{\mathrm{ox}} \varepsilon_{0}},
$$

where $Q_{\mathrm{f}}$ is the fixed charge near the $\mathrm{Si}-\mathrm{SiO}_{2}$ interface, $T_{\mathrm{Ox}}$ is the oxide thickness, $K_{\mathrm{ox}}$ is the oxide dielectric constant and $\varepsilon_{0}$ is the permittivity of vacuum.

Figure 10.2a shows a plot of $V_{\mathrm{FB}}$ versus oxide thickness for thermally 


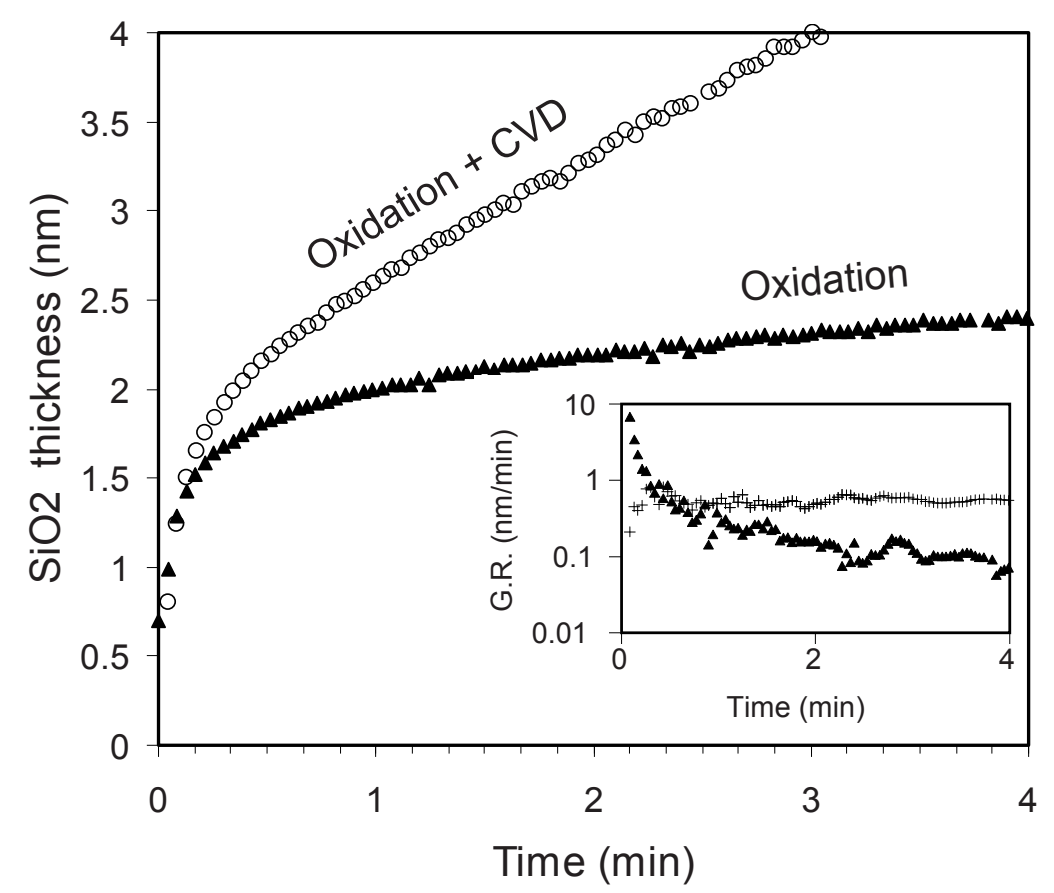

Figure 10.1: In situ thickness measurements without $(\mathbf{\Lambda})$ and with (o) silane $(0.08 \%)$; other conditions were the same. In the inset, the growth rates are shown for the CVD $(+)$ and oxidation $(\boldsymbol{\Delta})$ components. 

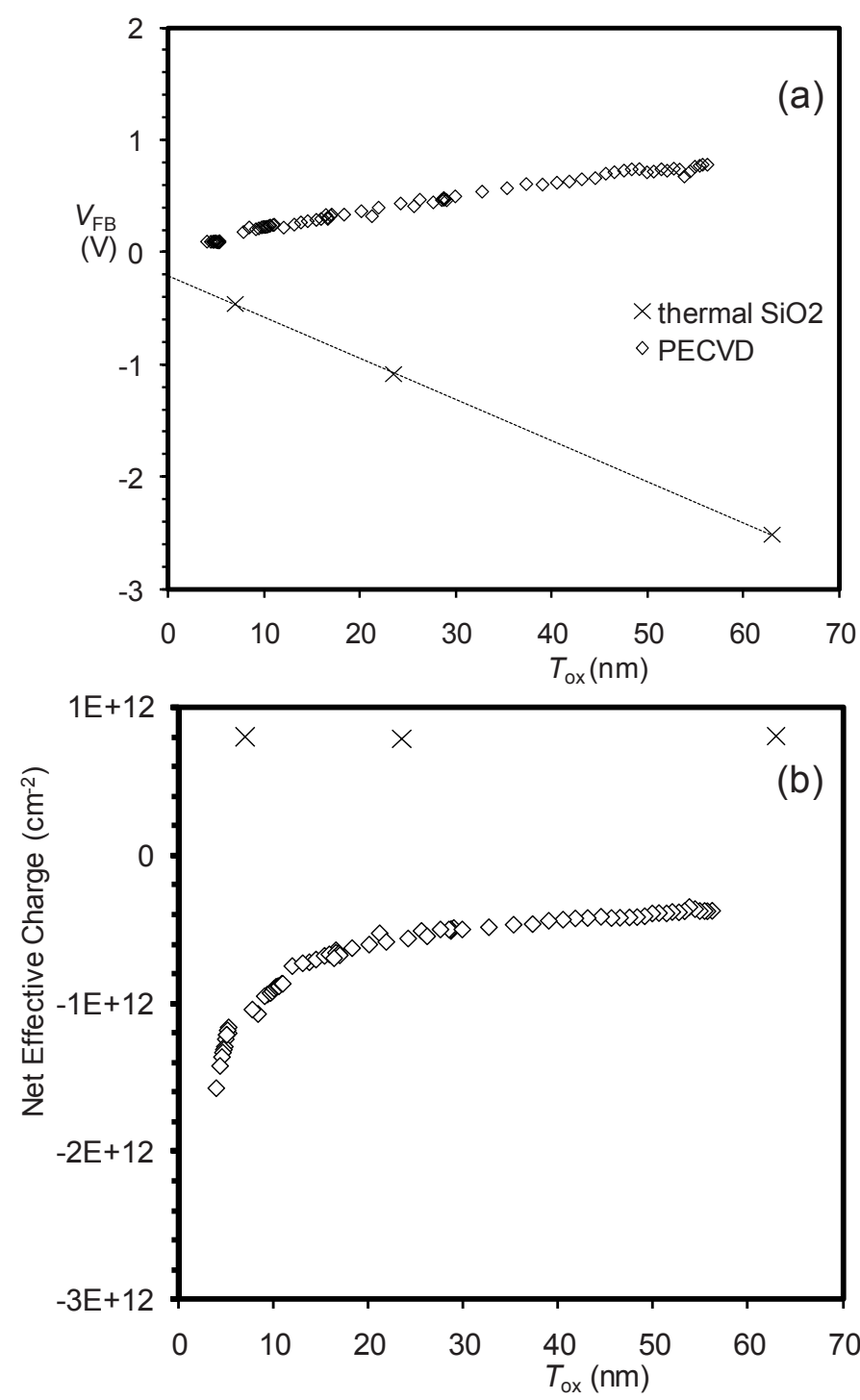

Figure 10.2: Flatband voltage (a) and Net Oxide charge (b) plotted versus oxide thickness; n-type substrates and Al-gates; after PMA. $(\times)$ Reference oxides grown by dry oxidation at $950^{\circ} \mathrm{C}$ followed by a POA (reference); $(\diamond)$ PECVD oxides. The slope of the dotted line is proportional to the fixed charge while the intercept equals $\varphi_{\mathrm{ms}}$. 


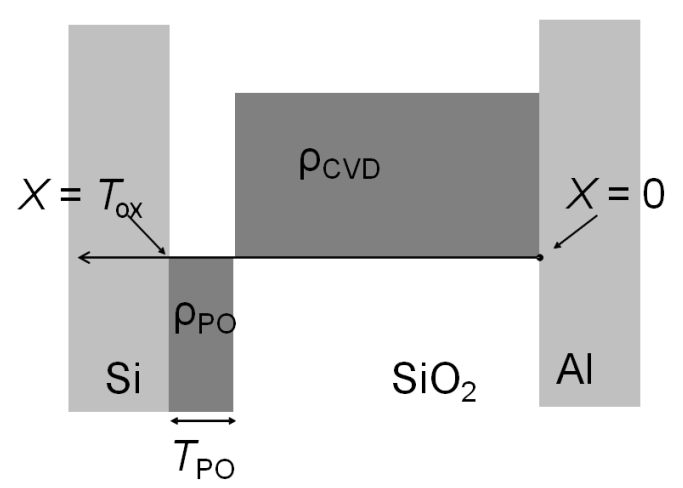

Figure 10.3: Graphical representation of the double-layer model.

grown oxides, and for our PECVD layers. If we compare the curves of thermal oxides and PECVD oxides in Figure 10.2a, we observe different signs of the slopes, indicating positive and negative net-charges. The data set of thermally grown oxide has a slope of $-Q_{\mathrm{f}} T_{\mathrm{Ox}} / K_{\mathrm{ox}} \varepsilon_{0}$ and an intercept on the $V_{\mathrm{FB}}$ axis of $\varphi_{\mathrm{ms}}=-0.214 \mathrm{~V}\left(Q_{\mathrm{f}}\right.$ is assumed to be the same and near the Si interface for all data points) [207]. The $\varphi_{\mathrm{ms}}$ thus obtained can be used to calculate $Q_{\mathrm{f}}$. These values are shown in Figure $10.2 \mathrm{~b}$. A positive charge of $8 \cdot 10^{11} \mathrm{~cm}^{-2}$ was calculated for the thermally grown oxide (see crosses in Figure 10.2b). If $Q_{\text {ox }}$ is calculated for the given PECVD oxides using the expression (8.8), one finds that thinner layers contain a higher amount of negative charge compared to thicker films (see open diamonds in Figure 10.2b). We attribute this important and novel result to the initial plasma oxidation step, which cannot be avoided. Thus, the plasma-oxidized region near the Si interface becomes dominant for thinner layers. This plasma oxide may therefore be responsible for the negative charge formation.

The effect of the negatively charged plasma oxide on flatband voltage can be described by equation (8.7). To solve the integral, we assume a homogeneous distribution of the negative charge density $\left(\rho_{\mathrm{PO}}\right)$ in oxide volume between the silicon interface, $x=T_{\mathrm{Ox}}$, and $x=T_{\mathrm{PO}}$, where $T_{\mathrm{PO}}$ is the thickness of the plasma-oxidized region (see Figure 10.3). Integrating reveals

$$
V_{\mathrm{FB}}^{\mathrm{PO}}=\frac{\rho_{\mathrm{PO}} T_{\mathrm{PO}}\left(2 T_{\mathrm{ox}}-T_{\mathrm{PO}}\right)}{2 C_{\mathrm{ox}} T_{\mathrm{ox}}}
$$

where $V_{\mathrm{FB}}^{\mathrm{PO}}$ is the flatband voltage change due to the plasma oxide. The second 
term simplifies to $Q_{\mathrm{PO}} / C_{\mathrm{ox}}$ when $T_{\mathrm{ox}}$ is much larger than $T_{\mathrm{PO}}\left(Q_{\mathrm{PO}}=\rho_{\mathrm{PO}}\right.$. $\left.T_{\mathrm{PO}}\right)$.

The pure CVD component adds the expected positive charge, thus compensating for the interface-located negative charge (the thicker the film, the more negative charge is compensated). To describe the effect of the positively charged CVD oxide on flatband voltage, we assume a homogeneous distribution of the positive charge density $\left(\rho_{\mathrm{CVD}}\right)$ in the volume between the gate, $x=0$, and $x=\left(T_{\mathrm{ox}}-T_{\mathrm{PO}}\right)$, see Figure 10.3. Integrating equation (8.7) reveals

$$
V_{\mathrm{FB}}^{\mathrm{CVD}}=\frac{\rho_{\mathrm{CVD}}\left(T_{\mathrm{ox}}-T_{\mathrm{PO}}\right)^{2}}{2 C_{\mathrm{ox}} T_{\mathrm{ox}}},
$$

where $V_{\mathrm{FB}}^{\mathrm{CVD}}$ is the flatband voltage change due to the CVD oxide. The second term simplifies to $Q_{\mathrm{CVD}} / 2 C_{\mathrm{ox}}$ for $T_{\mathrm{Ox}} \gg T_{\mathrm{PO}}\left(Q_{\mathrm{CVD}}=\rho_{\mathrm{CVD}} \cdot T_{\mathrm{Ox}}\right)$. Uniform charge distribution can occur if defects, such as silicon dangling bonds or plasma damage, are continuously added to the depositing oxide.

The effect of the two layers on the flatband voltage can now be calculated. The double layer model fits our data (open diamonds in Figure 10.4a) given a negative oxide charge density $\rho_{\mathrm{PO}}$ of $2.3 \cdot 10^{18} \mathrm{~cm}^{-3}$ within $T_{\mathrm{PO}}=2.5$ $\mathrm{nm}$, and a positive space charge density $\rho_{\mathrm{CVD}}$ of $9 \cdot 10^{16} \mathrm{~cm}^{-3}$.

We performed additional experiments to confirm our double layer model. These are presented in Figure 10.4. The results of thermally grown oxide and our standard PECVD process are included in Figure 10.4 for reasons of clarity. In a first experiment, initial oxide was grown by plasma oxidation only, i.e., in $\mathrm{Ar}-\mathrm{N}_{2} \mathrm{O}$ plasma without $\mathrm{SiH}_{4}$. After 15 minutes of oxidation, silane was introduced into the system and the PECVD mode was activated. The prolonged plasma oxidation increased the flatband voltage (see open triangles in Figure 10.4a). Our model described this effect by an increase in $T_{\mathrm{PO}}$ from 2.5 to $2.9 \mathrm{~nm}$ with the same negative $\rho_{\mathrm{PO}}$ of $2.3 \cdot 10^{18} \mathrm{~cm}^{-3}$, leaving the $\rho_{\mathrm{CVD}}$ unchanged (i.e. $9 \cdot 10^{16} \mathrm{~cm}^{-3}$ ). The latter indicated that the pure CVD mode was not influenced.

In a second experiment, we performed PECVD on a 7-nm thick thermally grown $\mathrm{SiO}_{2}$ to minimize the influence of the initial plasma oxidation step (see plus signs in Figure 10.4a and Figure 10.4b). We obtained much less negative-charge $\left(\rho_{\mathrm{PO}}\right.$ of $\left.5 \cdot 10^{17} \mathrm{~cm}^{-3}\right)$ again without changing $\rho_{\mathrm{CVD}}$, which reflected a similar trend of the flatband voltage in relation to oxide thickness. However, the net effective charge was still negative indicating that plasma oxidation could not be ruled out completely.

The negative charge can be reduced during POA (see solid circles in Figure 10.4b), resulting in a net effective positive charge for the thicker layers $(15-50 \mathrm{~nm})$. However, the charge for thinner layers remains negative. 

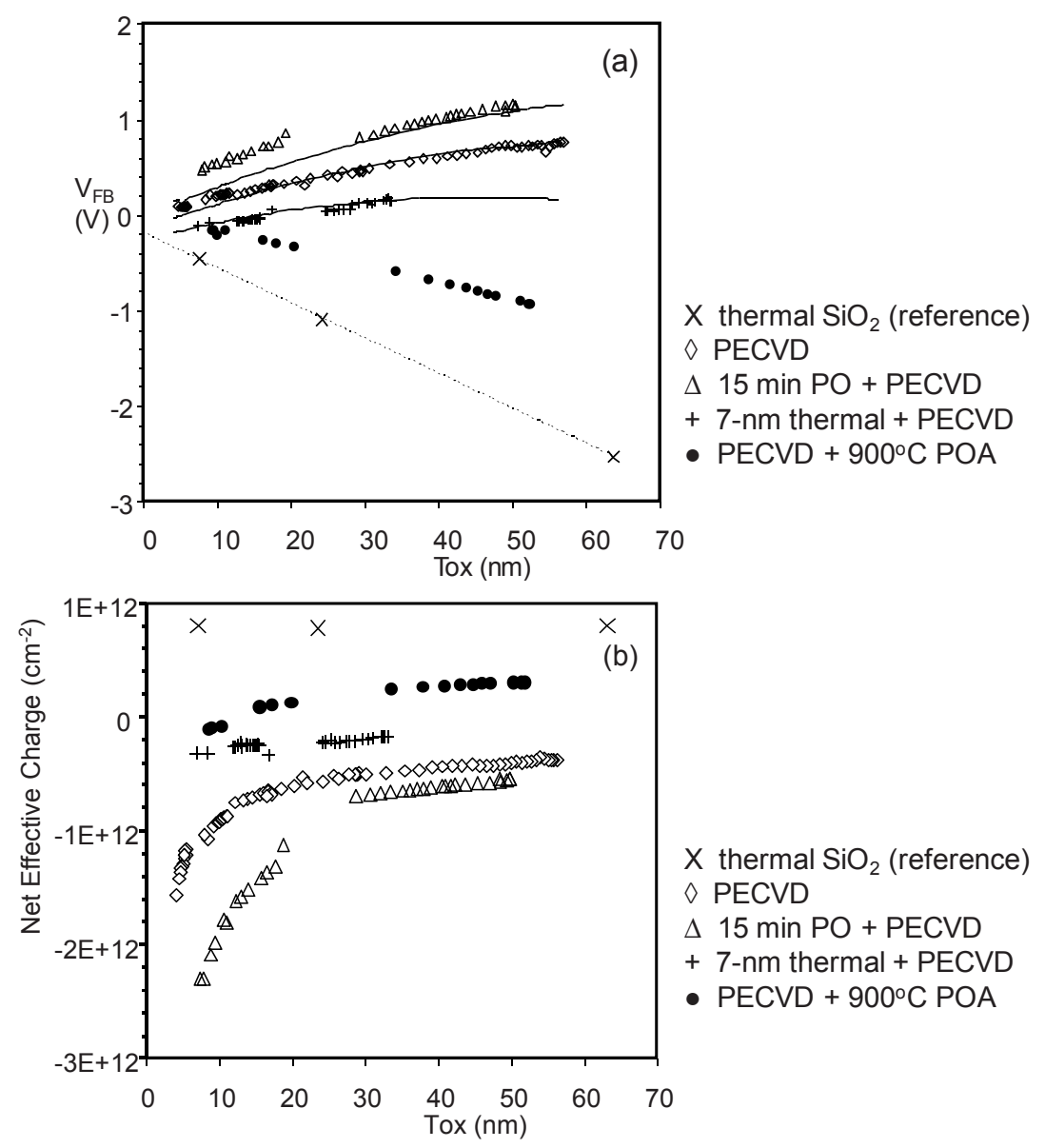

Figure 10.4: Flatband voltage (a) and Net Oxide charge (b) plotted versus oxide thickness; n-type substrates and Al-gates; after PMA. $(\times)$ Reference oxides grown by dry oxidation at $950^{\circ} \mathrm{C}$ followed by a POA (reference); $(\diamond)$ PECVD oxides; $(\triangle)$ 15 min plasma oxidation followed by PECVD; $(+)$ PECVD oxides deposited on 7-nm thick thermal $\mathrm{SiO}_{2} ;(\bullet) \mathrm{PECVD}$ oxides with POA and PMA. The slope of the dotted line is proportional to the fixed charge while the intercept equals $\varphi_{\mathrm{ms}}$. The solid lines represent fitting based on our double-layer model (see text). 
Negative effective charges were reported occasionally in PECVD silicon oxides when relatively thin layers $(10-50 \mathrm{~nm})$ were deposited in highly-diluted plasmas at low deposition rates [208-210]. Negative charges were also reported for silicon dioxide layers grown solely by plasma oxidation [211,212]. These publications support our conclusion on the influence of plasma oxidation on the oxide charge that always occurs parallel to deposition. With this in mind, a detailed study and model of oxidation mechanisms is needed. The majority of the PECVD oxide layers are deposited at much higher rates, and films are usually (considerably) thicker. These conditions are expected to minimize the influence of plasma oxidation, and a positive oxide charge is likely to be measured.

\subsubsection{The electronic nature of the negative charge}

Additional measurements showed that it was impossible to detrap the negative charge by applying negative voltage on the gate. This indicates that charge traps that accumulate negative charge in our material have energies in the $\mathrm{SiO}_{2}$ band gap anywhere below the Fermi level of a substrate, see Figure 10.5. For n-type $\mathrm{Si}$ (the experiments described above), the electron traps may lie in (i) the Si band gap (blue levels), or (ii) in the $\mathrm{Si} / \mathrm{SiO}_{2}$ valence band offset (red levels). So, the first thing one should do would be to narrow down this energy interval. If these traps were in (i), then no negative charge accumulation would be observed on p-type substrates. Figure 10.6 however indicates that the negative charge in $\mathrm{SiO}_{2}$ persists on p-type substrates. Therefore, one should conclude that the charge trap levels are in the $\mathrm{Si} / \mathrm{SiO}_{2}$ valence band offset region $\left(\Delta E_{\mathrm{V}}=4.4\right.$ $\mathrm{eV}$ [213]), i.e., below the Si valence band edge (red levels).

The valence-band offsets for $\mathrm{Si} / \mathrm{SiO}_{2}$ could be studied in more detail by various analytical techniques (see chapter 7 . Unfortunately, we had to leave that as future work.

\subsubsection{The physical nature of the negative charge}

Negatively charged oxygen atoms could cause the negative charge due to plasma oxidation. This assumption is addressed below.

During the last two decades, a number of theoretical models of silicon oxidation in the ultra-thin regime were constructed in order to surpass limitations of the commonly used Deal-Grove model. The Beck-Majkusiak model gives precise predictions even for ultrathin oxide thickness regime, for both classical oxidation in furnace, and for processing in rapid thermal oxidation (RTO) reactor, and is consistent with description of plasma oxidation processes [214]. The Beck-Majkusiak model assumes that the oxidation rate in 


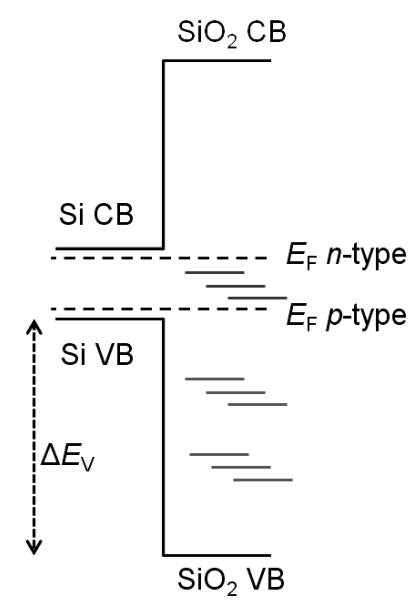

Figure 10.5: Energy band diagram of the $\mathrm{Si}-\mathrm{SiO}_{2}$ structure under flatband conditions.

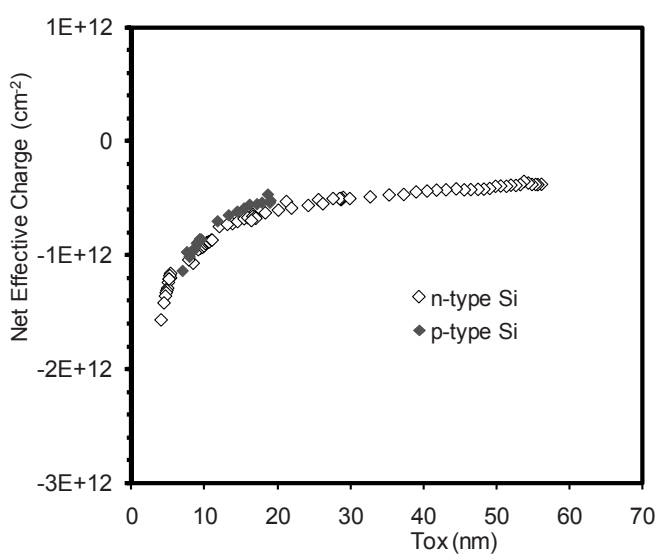

Figure 10.6: Net Oxide charge plotted versus oxide thickness; n-type and p-type substrates and Al-gates; after PMA. 
the first phases is limited by equilibrium between the flux of electron tunneling or thermo emission, which ionizes the oxygen atoms at the $\mathrm{SiO}_{2}$ surface, and the flux of the ionized species that flow through the already grown oxide. This means that the volume of $\mathrm{SiO}_{2}$ should be full of negatively ionized oxygen atoms. In the model, the charge of the negative ions is not included in the fitted $Q_{\text {eff }}$, it is included in the expression for the ion flux $F_{\mathrm{i}}(x)$. If one suddenly froze this distribution, the total effective charge density could then be negative. This cannot be confirmed experimentally for thermal oxidation since the high temperature will also act as an anneal that reduces the negative charge (this was also true for our PECVD layers, which showed positive charge after POA, see solid circles in Figure 10.4b). In our experiments without POA, however, the temperature is much lower, which might visualize this "reezing" effect.

The Beck-Majkusiak model relates existence of this negative charge to a surplus of oxygen in the PO layer. The question arises how this extra oxygen is incorporated in the PO layer. The surplus oxygen could be deposited as over-stoichiometric $\mathrm{SiOx}$ (with $x>2$ ). The extra oxygen can be incorporated e.g. in the form of a peroxy bridge $\left(\mathrm{O}_{3} \equiv \mathrm{Si}-\mathrm{O}-\mathrm{O}-\mathrm{Si} \equiv \mathrm{O}_{3}\right)$ [215], or non-bridging $\mathrm{O}$ atom (i.e., formation of two $\mathrm{O}_{3} \equiv \mathrm{Si}-\mathrm{O} \cdot \cdot \mathrm{O}-\mathrm{Si} \equiv \mathrm{O}_{3}$ dangling bonds instead of one $\mathrm{O}_{3} \equiv \mathrm{Si}-\mathrm{O}-\mathrm{Si} \equiv \mathrm{O}_{3}$ bond). It is known that over-stoichiometric SiOx does not exist in thermal oxidized layers [215], besides some peroxy bridges in very low concentration. Over-stoichiometric $\mathrm{SiOx}$ is not stable at typical temperatures for thermal oxidation. It could however exist in our experiments because of the lower temperatures. This is supported by earlier data in our laboratory on plasma deposition of $\mathrm{SiO}_{2}$ films at $100^{\circ} \mathrm{C}$, where the authors measured a correlation between the negative charge and the O/Si ratio (by XPS) [209]. Namely, the thin films mostly exhibited negative charge and the O/Si ratio was slightly higher than 2. This assumption is also in accordance with the work of Afanas'ev and Stesmans, who mentioned $\mathrm{O}_{3} \equiv \mathrm{Si}-\mathrm{OH}$ centers (created by irradiation with $10 \mathrm{eV}$ photons) as neutral traps for electrons [216].

The surplus oxygen could also form $\mathrm{O}_{2}^{-}$molecules. Computations of Ewig and Tellinghuizen showed that $\mathrm{O}_{2}^{-}$is stable against electron autodetachment in ionic crystals [217]. Although our oxide is amorphous, we speculate that the $\mathrm{O}_{2}^{-}$molecules are also stable within the silica network. They could remain from the diffusion of oxygen through $\mathrm{SiO}_{2}$ towards the interface of the Si substrate, in line with the Beck-Majkusiak model. Salh, Von Czarnowski, and Fitting have studied over-stoichiometric $\mathrm{SiOx}$ by cathodoluminescence (CL) $[173,218,219]$. To do so, they implanted dry oxidized $\mathrm{SiO}_{2}$ layers additionally by $\mathrm{O}^{+}$ions. The surprising feature of these samples appeared in their multiple regular-shaped spectra in the green-near IR (500-820 $\mathrm{nm}$ ) region. The sub-band 
positions corresponded to almost equidistant energy steps of about $120 \mathrm{meV}$. From [217] they associated their findings to absorption and emission spectra of $\mathrm{O}_{2}^{-}$molecules and discussed them thoroughly in [218]. Thus they could explain the multimodal luminescence spectra of $\mathrm{SiO}_{2}: \mathrm{O}$ by electronic-vibronic transitions of $\mathrm{O}_{2}^{-}$molecules in terms of a configuration coordinate model.

\subsection{3 $C-V$ measurements of $\mathrm{SiO}_{2}: \mathrm{O}$ layers}

Ideally, the presence of $\mathrm{O}_{2}^{-}$(or any other form of negatively charged oxygen) must induce a shift in flatband voltage as measured by $C-V$ measurements. Therefore, a cooperation was started between Salh and Fitting of Rostock University and the author of this thesis. The aim was to proof electrically that the implantation of oxygen could induce a negative charge in the bulk oxide (contrary to our results that showed a negative charge at the interface). The next phase should be to measure our oxides by CL, verifying the multimodal spectra in our oxides at the interface. The first part $\left(C-V\right.$ measurements of $\mathrm{SiO}_{2}: \mathrm{O}$ layers) is presented below. The second part was unfortunately not conducted so far. To perform the first part, Sahl delivered the oxygen-implanted samples, while the $C-V$ measurements were performed in our laboratory. The $\mathrm{SiO}_{2}-$ layers prepared by dry oxidation ( $214 \mathrm{~nm}$ thick) where implanted at $45 \mathrm{keV}$ with $\mathrm{O}^{+}$at doses of $10^{16} \mathrm{~cm}^{-2}, 5 \cdot 10^{16} \mathrm{~cm}^{-2}$ and $10^{17} \mathrm{~cm}^{-2}$. Afterwards, they were annealed in $\mathrm{N}_{2}$ at $1000^{\circ} \mathrm{C}$ for $30 \mathrm{~min}$. Processing at Philips Res. Labs. \& MESA+ involved: deposition of $\mathrm{Al}$ through shadow masks to define capacitors, and Post Metal Anneal at $400^{\circ} \mathrm{C} @ 10 \mathrm{~min}$ in humid $\mathrm{N}_{2}$ (cf. section 8).

We will start our discussion by describing the distribution of the implanted ions. The next step will be to formulate an expression that can be used to calculate the influence of the oxide charges on the measured flatband voltage, $V_{\mathrm{FB}}$. It should account for $Q_{\mathrm{f}}$ at $\mathrm{Si}-\mathrm{SiO}_{2}$ interface, and for $Q_{\text {bulk }}$ according to the implantation profile. finally, the measurements will be presented.

According to the Lindhard, Scharff, and Schiott (LSS) model [220], the ion concentration as a function of depth, $\rho_{\text {ion }}(x)$, in amorphous materials can be described by a Gaussian curve:

$$
\rho_{\text {ion }}(x)=\frac{\theta}{\sqrt{2 \pi} \Delta R_{\mathrm{p}}} \exp \left(-\frac{\left(x-R_{\mathrm{p}}\right)^{2}}{2 \Delta R_{\mathrm{p}}^{2}}\right),
$$

where $\theta$ is the implantation dose (ions $/ \mathrm{cm}^{2}$ ), $\Delta R_{\mathrm{p}}$ is the standard deviation of the Gaussian distribution (also known as projectred straggle), and $R_{\mathrm{p}}$ is the projected range (see Figure 10.7). $R_{\mathrm{p}}$ and $\Delta R_{\mathrm{p}}$ can be calculated from the mass of the implanted ions and the target atoms, and the mass density of the target material [220]. The results of these calculations (using Silvaco TCAD tools) 
Table 10.1: Calculated parameters of the implantation distribution of $\mathrm{O}^{+}$in amorphous $\mathrm{SiO}_{2}$.

\begin{tabular}{llll}
\hline Implantation energy & $45 \mathrm{keV}$ & & \\
\hline$\theta$ (ions $\left./ \mathrm{cm}^{2}\right)$ & $10^{16}$ & $5 \cdot 10^{16}$ & $10^{17}$ \\
$R_{\mathrm{p}}(\mathrm{nm})$ & 100 & & \\
$\Delta R_{\mathrm{p}}(\mathrm{nm})$ & 36 & & \\
$\rho_{\text {ion }}\left(x=R_{\mathrm{p}}\right)\left(\mathrm{cm}^{-3}\right)$ & $1.1 \cdot 10^{21}$ & $5.6 \cdot 10^{21}$ & $1.1 \cdot 10^{22}$ \\
\hline
\end{tabular}

are summarized in Table 10.1, and plotted in Figure 10.7. This table and figure show that the maximum of the distribution can be found at approx. half the oxide thickness. Please note that the $\mathrm{SiO}_{2}-\mathrm{Si}$ interface, located at $0.214 \mu \mathrm{m}$, is not included in the simulation. Oxygen atoms will be implanted at substantial concentrations into the $\mathrm{Si}$, but their exact distribution in the silicon is not relevant for the considerations we make. It is important however to be aware of the effect, because such concentrations (around $5 \cdot 10^{19} \mathrm{~cm}^{-3}$ at the $\mathrm{SiO}_{2}-\mathrm{Si}$ interface) are likely to deteriorate the interface and quality of $\mathrm{Si}$, and might thus affect $C-V$ measurements. On the other hand, the crystalline damage and the interface will be restored by the anneal at $1000^{\circ} \mathrm{C}$ for $30 \mathrm{~min}$ [1]. Nevertheless, the results would have been more straightforward if a lower implantation energy (e.g., $20 \mathrm{keV}$ ) would have been chosen.

The expression used to calculate the influence of the oxide charges on $V_{\mathrm{FB}}$ should account for $\rho_{\mathrm{f}}\left(Q_{\mathrm{f}}=\rho_{\mathrm{f}} \cdot T_{\mathrm{f}}\right)$ at $\mathrm{Si}-\mathrm{SiO}_{2}$ interface, and for $\rho_{\text {bulk }}\left(Q_{\text {bulk }}=\right.$ $\left.\rho_{\text {bulk }} \cdot T_{\mathrm{ox}}\right)$ according to the implantation profile. The effect of the fixed charge, $\rho_{\mathrm{f}}$, on the flatband voltage can be calculated using equation (10.2). To describe the effect of the negatively charged CVD oxide, $\rho_{\text {bulk }}$, on flatband voltage, we insert the implantation profile (equation (10.4)) of the oxygen atoms $\left(\rho_{\text {bulk }}\right)$ into equation (8.7) (see Figure 10.8). Integrating reveals

$$
\begin{aligned}
& V_{\mathrm{FB}}^{\text {bulk }}=-\frac{1}{2} \theta\left[-\sqrt{2} \Delta R_{\mathrm{p}} \mathrm{e}^{-R_{\mathrm{p}}^{2} / \Delta R_{\mathrm{p}}^{2}}-\sqrt{\pi} R_{\mathrm{p}} \operatorname{erf}\left(\frac{\sqrt{2} R_{\mathrm{p}}}{2 \Delta R_{\mathrm{p}}}\right)\right. \\
& \left.+\sqrt{2} \Delta R_{\mathrm{p}} \mathrm{e}^{-\left(R_{\mathrm{p}}-T_{\mathrm{ox}}\right)^{2} / \Delta R_{\mathrm{p}}^{2}}+\sqrt{\pi} R_{\mathrm{p}} \operatorname{erf}\left(\frac{\sqrt{2}\left(R_{\mathrm{p}}-T_{\mathrm{ox}}\right)}{2 \Delta R_{\mathrm{p}}}\right)\right] / \sqrt{\pi} C_{\mathrm{ox}} T_{\mathrm{ox}}
\end{aligned}
$$

However, it is assumed that only a fraction, $f(\theta)$, of the implanted ions is electrically active as $\mathrm{O}_{2}^{-}$. So, a small change in the expression is necessary: $\theta$ is to be replaced by $f(\theta) \times \theta$.

The $C-V$ curves are shown in Figure 10.9. The figure reveals that $V_{\mathrm{FB}}$ 


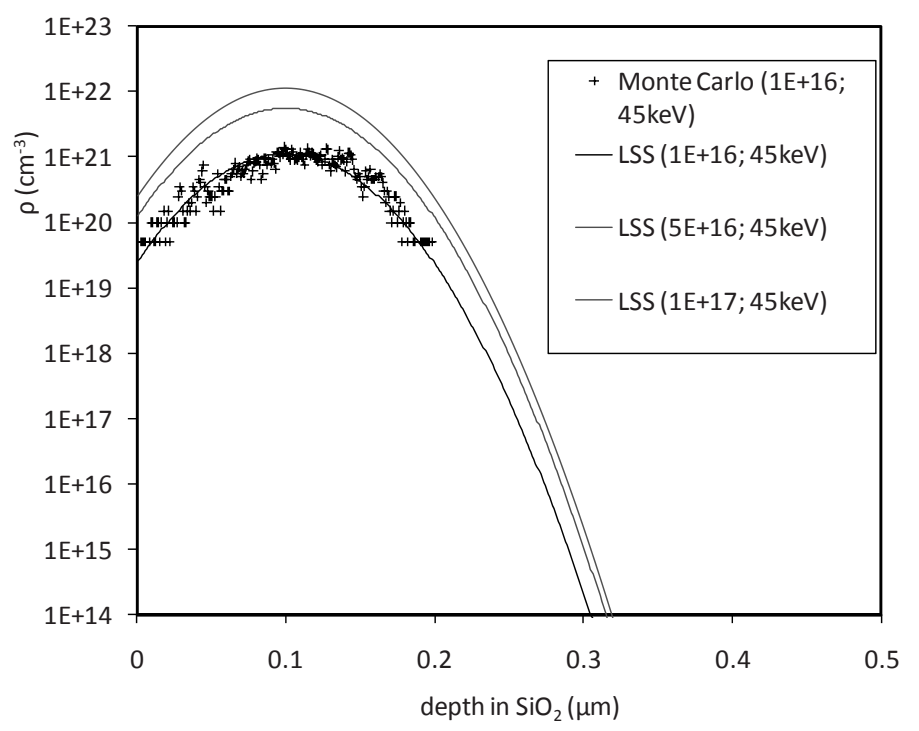

Figure 10.7: The implantation profile of $\mathrm{O}^{+}$in amorphous $\mathrm{SiO}_{2}$. Profiles are shown for both LSS model and Monte Carlo simulations.

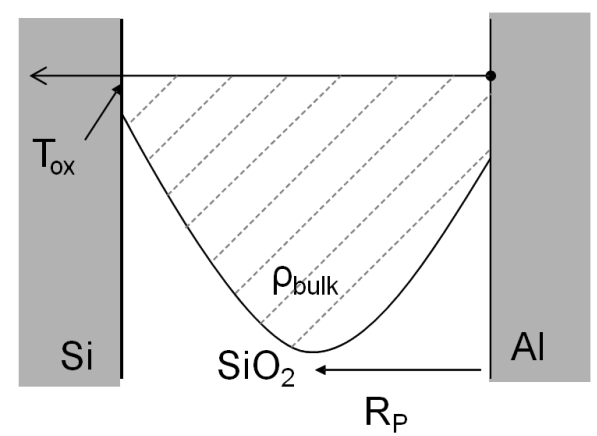

Figure 10.8: Graphical representation of the charge distribution in the $\mathrm{Si}_{2} \mathrm{O}: \mathrm{O}$ layer. 


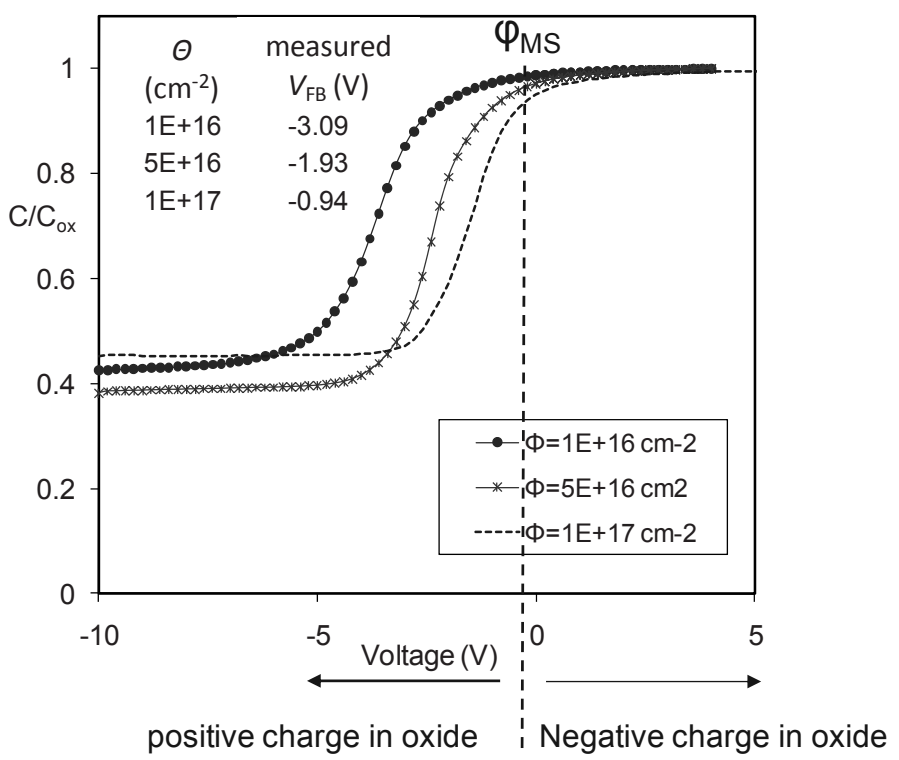

Figure 10.9: $\mathrm{C}-V$ curves of $\mathrm{SiO}_{2}: \mathrm{O}$ layers.

of the $\mathrm{SiO}_{2}$ : O films is more negative than $\varphi_{\mathrm{m}} \mathrm{s}$. This means that these films have a positive effective charge (cf. equation (8.8) and Figure 10.9). However, $V_{\mathrm{FB}}$ shifts towards $\varphi_{\mathrm{ms}}$ for higher implantation doses. This means that the effective positive charge is reduced when the implantation dose is increased. This observation can be explained easily. Thermal $\mathrm{SiO}_{2}$ adds a positive charge $\left(Q_{\mathrm{f}}\right)$ near the $\mathrm{Si}-\mathrm{SiO}_{2}$ interface. This $Q_{\mathrm{f}}$ is partly compensated by a negative charge of oxygen molecules in the bulk oxide. A higher implantation dose will reduce the effective positive charge to lower values.

The effect of the implantations on the flatband voltage can also be calculated (by combining equations (10.2) and (10.5). The results are shown in Table 10.2. Our model can describe the measured flatband voltages by a constant $Q_{\mathrm{f}}$ of $3.2 \cdot 10^{11} \mathrm{~cm}^{-2}$ for all samples. This indicates that the anneal equally restored the $\mathrm{Si}-\mathrm{SiO}_{2}$ interface for all samples. This $Q_{\mathrm{f}}$ is partly compensated by negatively charged oxygen atoms. According to our model, only a small fraction (about $5 \cdot 10^{-6}$ ) of the implantation dose is electrically activated. The negative-charge distribution can easily be found from Figure 10.7 by multiplying $\frac{1}{2} \cdot \rho_{\text {bulk }}$ by the activation fraction from Table $10.2\left(\frac{1}{2}\right.$ is added because 
Table 10.2: Calculated $V_{\mathrm{FB}}$ and $Q_{\mathrm{f}}$ as a function of the oxygen implantation dose in amorphous $\mathrm{SiO}_{2}$.

\begin{tabular}{lrrr}
\hline$\theta\left(\right.$ ions $\left./ \mathrm{cm}^{2}\right)$ & $1 \cdot 10^{16}$ & $5 \cdot 10^{16}$ & $1 \cdot 10^{17}$ \\
\hline measured $V_{\mathrm{FB}}(\mathrm{V})$ & -3.09 & -1.93 & -0.94 \\
modelled $V_{\mathrm{FB}}(\mathrm{V})$ & -3.08 & -1.92 & -0.93 \\
$f(\theta)(-)$ & $6.3 \cdot 10^{-6}$ & $5.3 \cdot 10^{-6}$ & $4.4 \cdot 10^{-6}$ \\
$Q_{\mathrm{f}}\left(\mathrm{cm}^{-2}\right)$ & & $3.2 \cdot 10^{11}$ & \\
\hline
\end{tabular}

2 implanted oxygen atoms are needed to form one $\mathrm{O}_{2}^{-}$molecule). The highest concentration (according to our model) of $\mathrm{O}_{2}^{-}$is $2 \cdot 10^{16} \mathrm{~cm}^{-3}$ (at $R_{\mathrm{p}}$ for $\theta=1 \cdot 10^{17}$ ions $/ \mathrm{cm}^{2}$ ). This is just below the maximum solubility of $\mathrm{O}_{2}$ in $\mathrm{SiO}_{2}$, $5 \cdot 10^{16} \mathrm{~cm}^{-3}$ [221], and is therefore a physically feasible and relevant number.

\subsubsection{Preliminary conclusions and recommendations}

Silicon dioxide films were implanted with oxygen. Net oxide charge was positive, and was a function of implantation dose. A model that assumes a positive fixed charge at the interface $\left(3.2 \cdot 10^{11} \mathrm{~cm}^{-2}\right)$ and a distribution of the negative charge similar to the implantation profile of the bulk oxide layer can describe the results. Further verification steps remain to be done. These should include a series of experiments performed at similar conditions, but at lower implantation energies; inclusion of non-implanted samples into the experiments (to verify the modeled $Q_{\mathrm{f}}$ ). Lastly, perform CL-measurements on our PECVD oxides to verify that the multimodal peaks in CL spectra also appear at locations where we assume a negative charge. Another topic that would be interesting to study via $I-V$ measurements: can implanted oxygen create a trap-related conduction mechanism, similar to those observed in Figure 9.1?

\subsection{Conclusions}

The net charge of our $0.08 \% \mathrm{SiH}_{4}-1 \mathrm{~Pa}-150^{\circ} \mathrm{C}$ silicon dioxides was found to be negative, and was a function of layer thickness. This was explained by plasma oxidation (PO) of silicon, which added a negative charge $\left(2.3 \cdot 10^{18} \mathrm{~cm}^{-3}\right)$ to the interfacial oxide layer ( $2.5 \mathrm{~nm}$ thick), while the CVD component added a nearly homogeneous distribution of positive charge $\left(9 \cdot 10^{16} \mathrm{~cm}^{-3}\right)$ to the bulk oxide.

The charge traps of the negative-charged PO layer are located in the $\mathrm{Si} / \mathrm{SiO}_{2}$ valence band offset region. 
The implantation of oxygen into thermally grown $\mathrm{SiO}_{2}$ adds a negative charge to the bulk oxide layer. The effect of the negative charge on the flatband voltage can be described by the implantation profile. 


\subsection{Conclusions}

We have deposited $\mathrm{SiO}_{2}$ gate dielectric layers at $150^{\circ} \mathrm{C}$ by remote plasmaenhanced CVD. The aim of this work was to contribute to the fabrication of electronic devices on top of a complete integrated circuit, which follows the contemporary ideas of 3-D integration. To keep the circuit intact, the substrate temperature should remain below $400^{\circ} \mathrm{C}$.

A remote ICPECVD system was build for the deposition of thin $\mathrm{SiO}_{2}$ dielectric films. The plasma was characterized by a Langmuir probe and optical emission spectroscopy. We obtained electron densities and electron energy distribution functions (EEDF) by a Langmuir probe for $\mathrm{Ar}, \mathrm{Ar}-\mathrm{N}_{2} \mathrm{O}$ and $\mathrm{Ar}-\mathrm{N}_{2}$ plasmas at several probe locations in the chamber. The results of these measurements were used to model plasma processes.

Measured EEDFs can be divided in two parts: a large part of "slow" electrons, which exhibit Maxwell-Boltzmann energy distributions, and a smaller part $\left(\sim 10^{-3}\right)$ of "fast" electrons in the energy range of 20 to $50 \mathrm{eV}$, deviating from Maxwell-Boltzmann distribution.

Addition of small amounts of $\mathrm{N}_{2}(5-10 \%)$ or $\mathrm{N}_{2} \mathrm{O}(18 \%)$ to the Ar plasma did not change the shape of the fast-electron tail of the EEDF, but decreased the densities of slow electrons by approximately a factor of two. Furthermore, adding silane to an argon plasma did not affect the fast-electron tail when the $\mathrm{SiH}_{4}$ concentration was below 8 vol. $\%$, this also not significantly affected the slow electrons when the $\mathrm{SiH}_{4}$ concentration was kept below 0.8 vol.\%.

Optical emission spectroscopy (OES) was used to verify the EEDF mea- 
surements as obtained by the Langmuir probe. It was demonstrated that line intensity ratios, as measured with OES, could only be explained when the measured EEDF was used. Therefore, it was concluded that the EEDFs were measured correctly, including the fast-electron tail. The tail significantly influences the plasma composition; ignoring the tail would cause strong disagreements between the actual and the modeled plasma processes.

Furthermore, the composition of $\mathrm{Ar}-\mathrm{N}_{2} \mathrm{O}-\mathrm{SiH}_{4}$ plasmas was modeled in the search for optimum process conditions in our ICP reactor. The measured EEDFs and electron densities were used to model plasma processes. The plasma composition was calculated for several total gas pressures and silane fractions in $\mathrm{Ar}-\mathrm{N}_{2} \mathrm{O}-\mathrm{SiH}_{4}$ plasmas. 173 reactions were considered. The process conditions were chosen such that large variations in plasma composition were achieved. The density of atomic oxygen was fairly constant for all process conditions. The density of several silane radicals and atomic hydrogen however changed radically for the different conditions. Relations were drawn between plasma-densities of Si-containing radicals and atomic hydrogen, and the expected physical and electrical properties of the films.

Silicon dioxide films were deposited at the same plasma conditions as were used for the modeling. The films were characterized by spectroscopic ellipsometry (SE), and by current-voltage $(I-V)$ and capacitance-voltage $(C-V)$ measurements. We obtained a good correlation of measured film properties with the simulated radical fluxes towards the substrate. These results indicated that our model is capable of predicting trends in some relevant film growth parameters. As such, the combination of plasma characterization, plasma modeling, and electrical measurements was demonstrated to be valuable tools for studying, predicting and optimizing the PECVD technology.

The optimization led to the deposition of films with high dielectric quality at a deposition temperature as low as $150^{\circ} \mathrm{C}$. The films exhibited very low leakage currents at the electric field strength of $6.5 \mathrm{MV} / \mathrm{cm}$, which was comparable to the leakage currents of thermally grown oxides. A very low midgap$D_{\text {it }}$ of $3 \cdot 10^{10} \mathrm{eV}^{-1} \mathrm{~cm}^{-2}$ was obtained. We showed their applicability to lowtemperature TFTs by demonstrating competitive mobility values and low offcurrents. The described low-temperature PECVD oxide can be further applied to low-temperature CMOS post-processing. This CMOS process can be employed for large-area TFTs as well as in 3-D electronics due to its low thermal budget.

The net charge of our best-quality oxides appeared to be negative and a function of layer thickness. It was demonstrated that two mechanisms contributed to the film growth and the formation of charges, namely plasma ox- 
idation of the silicon substrate and chemical vapor deposition. We suggested that the first nm-range of oxide thickness was formed by plasma oxidation that added a negative charge to the interfacial oxide layer, while the CVD component added a positive charge to the bulk oxide.

\subsection{Recommendations for further research}

The properties of thin dielectrics remain very important for the reliability of semiconductor devices. In line with this research, we think of the gate oxide in TFTs. It is well known that oxide defects are hazardous for a sufficient lifetime of the TFTs. Therefore, there is a continuous interest in the properties of thin dielectrics in general and the breakdown phenomenon in particular. The latter is the determining factor in oxide lifetime and was not treated this thesis. Further research should study the oxide breakdown and degradation in general by different test methods.

The negative fixed charge, located in the interfacial oxide layer, could be exploited in particular applications, e.g., the reduction of recombination losses in photovoltaic devices by electrostatically shielding the minority charge carriers using internal electric fields.

The negative charge could also be exploited to enhance the trade-off between the breakdown voltage and the on-resistance of lateral high-voltage devices. High-voltage devices usually require a thick and low-doped epitaxial layer, which makes them difficult to integrate with low-voltage circuitry. Because of the high-resistivity epitaxial layer, the on-state resistances of such devices is large. It has been shown that a lateral diode with a thin n-type epitaxial layer on a lowly doped p-substrate can give a higher breakdown voltage than a conventional lateral diode [222]. It is worthwhile to study if our plasma oxidation, thereby exploiting the negative fixed charge, can replace this n-type epitaxial layer. This would simplify and reduce the costs of the production process of these devices significantly. 
[1] S. Wolf and R.N. Tauber, "Thermal oxidation of single-crystal silicon," in Silicon processing for the VLSI Era. Vol. 1. Process technology, 1st ed Sunset Beach: Lattice Press, 1986, p. 198.

[2] S. Wolf and R.N. Tauber, "Chemical vapor deposition of amorphous and polycrystalline films," in Silicon processing for the VLSI Era. Vol. 1. Process technology, 1st ed Sunset Beach: Lattice Press, 1986, p. 161.

[3] S. Sedky, A. Witvrouw, H. Bender, and K. Baert, "Experimental determination of the maximum post-process annealing temperature for standard CMOS wafers," Electron Devices, IEEE Transactions on, vol. 48 (2), pp. 377-385, 2001.

[4] D.L. Smith, Thin-film deposition: principles and practice, 1st ed. New York: McGraw-Hill, 1995.

[5] F.S. Becker, D. Pawlik, H. Anzinger, and A. Spitzer, "Low-pressure deposition of high-quality $\mathrm{SiO}_{2}$ films by pyrolysis of tetraethylorthosilicate," Journal of Vacuum Science \& Technology B: Microelectronics and Nanometer Structures, vol. 5 (6), pp. 1555-1563, 1987.

[6] V.-A. Dao, V.-D. Nguyen, J. Heo, H. Choi, Y. Kim, N. Lakshminarayan, and J. Yi, "Effect of $\mathrm{N}_{2} \mathrm{O} / \mathrm{SiH}_{4}$ flow ratios on properties of amorphous silicon oxide thin films deposited by inductively-coupled plasma chemical vapor deposition with application to silicon surface passivation," Vacuum, vol. 84 (3), pp. 410-414, 2009.

[7] B.E. Deal and A.S. Grove, "General Relationship for the Thermal Oxida- 
tion of Silicon," Journal of Applied Physics, vol. 36 (12), pp. 3770-3778, 1965.

[8] http://www.cfdrc.com/;

http://www.fluent.com/solutions/examples/x128.htm;

http://www.semitech.us/consulting/pvt_growth/models;

http://uva.computationalscience.nl/;

http://www.eu-CrossGrid.org/;

http://www.lpp.fr/;

http://uigelz.eecs.umich.edu/.

[9] J. van Dijk, G.M.W. Kroesen, and A. Bogaerts, "Plasma modelling and numerical simulation," Journal of Physics D-Applied Physics, vol. 42 (19), Oct 2009 (special issue with a state of the art overview in plasma modeling and numerical simulation).

[10] W.N.G. Hitchon, D.J. Koch, and J.B. Adams, "An efficient scheme for convection-dominated transport," Journal of Computational Physics, vol. 83 (1), pp. 79-95, 1989.

[11] M. Surendra and D.B. Graves, "Particle simulations of radio-frequency glow discharges," IEEE Transactions on Plasma Science, vol. 19 (2), pp. 144-157, 1991.

[12] U. Kortshagen and L.D. Tsendin, Electron kinetics and applications of glow discharges vol. 367. New York [etc.]: Kluwer Academic Publishers, 2002, p. http:/ /dx.doi.org/10.1007/b115089.

[13] M.W. Kiehlbauch and D.B. Graves, "Modeling argon inductively coupled plasmas: The electron energy distribution function and metastable kinetics," Journal of Applied Physics, vol. 91 (6), pp. 3539-3546, 2002.

[14] C.K. Birdsall and A.B. Langdon, Plasma physics via computer simulation. Bristol [etc.]: IOP Publishing Ltd., 1991.

[15] M.J. Kushner, "Monte-carlo simulation of electron properties in rf parallel plate capacitively coupled discharges," Journal of Applied Physics, vol. 54 (9), pp. 4958-4965, 1983.

[16] F.F. Chen, Introduction to plasma physics and controlled fusion, 2nd ed. vol. 1: Plasma physics. New York [etc.]: Plenum press, 1984, p. 3.

[17] D.A. Gurnett and A. Bhattacharjee, Introduction to Plasma Physics. Cambridge: Cambridge University Press, 2005, p. 2.

[18] J.P. Goedbloed and S. Poedts, Principles of Magnetohydrodynamics with applications to laboratory and astrophysical plasmas, 1st ed. Cambridge [etc.]: Cambridge University Press, 2004, p. 3.

[19] O.A. Popov, high density plasma sources. Park Ridge Noyes, 1995.

[20] R. Engeln, S. Mazouffre, P. Vankan, I. Bakker, and D.C. Schram, “Plasma 
expansion: fundamentals and applications," Plasma Sources Science and Technology, vol. 11 (3A), p. A100, 2002.

[21] H. Conrads and M. schmidt, "Plasma generation and plasma sources," Plasma Sources Science and Technology, vol. 9 (4), pp. 441-454, 2000.

[22] M.A. Lieberman and R.A. Gottscho, "Design of high-density plasma sources for materials processing," in Plasma sources for thin film deposition and etching. vol. 18, M.H. Francombe and J.L. Vossen, Eds., ed San Diego: Academic Press, 1994, pp. 19, 104.

[23] I. Langmuir, "Oscillations in Ionized Gases," Proceedings of the National Academy of Sciences of the United States of America, vol. 14 (8), pp. 627-637, August 1928.

[24] G. Schmidt, Physics of high temperature plasmas. London, UK: Academic, 1979, p. xii, 408.

[25] C.K. Birdsall, "Particle-in-cell charged-particle simulations, plus Monte Carlo collisions with neutral atoms, PIC-MCC," Plasma Science, IEEE Transactions on, vol. 19 (2), pp. 65-85, 1991.

[26] W. Greiner, L. Neise, and H. Stöcker, Thermodynamics and statistical mechanics. New York [etc.]: Springer, 1995.

[27] B. Chapman, "Impingment flux," in Glow discharge processes, 1st ed New York [etc.]: Wiley-Interscience, 1980, p. 8.

[28] B. Chapman, "Sheath formation at a floating substrate," in Glow discharge processes, 1st ed New York [etc.]: Wiley-Interscience, 1980, pp. 53-57.

[29] M.A. Lieberman and A.J. Lichtenberg, Principles of plasma discharges and materials processing 1st ed. New York: John Wiley \& Sons, Inc., 1994, pp. 40-42, 46-48, 91, 92, 129-132, 157-161, 278, 279.

[30] N. Pottier, "Interaction between two charged particles," in Nonequilibrium statistical physics: linear irreversible processes, 1st ed Oxford [etc.]: Oxford University Press, 2010, p. 99.

[31] W.J.M. Brok, "Modelling of transient phenomena in gas discharges," Ph.D. thesis, Applied Physics, Technische Universiteit Eindhoven, Eindhoven, 2005, pp. 12, 13, 99, 100.

[32] G.G. Raju, Gaseous electronics: Theory and practice. Boca Raton: Taylor \& Francis, 2006, p. 109.

[33] A.J. v. Roosmalen, J.A.G. Baggerman, and S.J.H. Brader, Dry Etching for VLSI. New York: Plenum Press, 1991, pp. 62, 92.

[34] B. Chapman, Glow discharge processes, 1st ed. New York [etc.]: WileyInterscience, 1980. 
[35] B. Chapman, "Debye shielding," in Glow discharge processes, 1st ed New York [etc.]: Wiley-Interscience, 1980, pp. 59, 60.

[36] P.N. Wainman, M.A. Lieberman, A.J. Lichtenberg, R.A. Stewart, and C. Lee, "Characterization at different aspect ratios (radius/length) of a radio frequency inductively coupled plasma source," Journal of Vacuum Science and Technology A: Vacuum, Surfaces, and Films, vol. 13 (5), pp. 2464-2469, 1995.

[37] S. Ashida, C. Lee, and M.A. Lieberman, "Spatially averaged (global) model of time modulated high density argon plasmas," Journal of Vacuum Science \& Technology A: Vacuum, Surfaces, and Films, vol. 13 (5), pp. 2498-2507, 1995.

[38] Y.P. Raizer, Gas discharge physics. Berlin [etc.]: Springer-Verlag, 1991.

[39] G. Crolly and H. Oechsner, "Comparative determination of the electron temperature in Ar- and $\mathrm{N}_{2}$-plasmas with electrostatic probes, optical emission spectroscopy OES and energy dispersive mass spectrometry EDMS," European Physical Journal, Applied Physics, vol. 15 (1), pp. 49-56, 2001.

[40] J.A.G. Baggerman, R.J. Visser, and E.J.H. Collart, “Power dissipation measurements in a low-pressure $\mathrm{N}_{2}$ radio-frequency discharge," Journal of Applied Physics, vol. 76 (2), pp. 738-746, 1994.

[41] R. Fischer and V. Dose, "Electron energy distribution reconstruction in low-pressure helium plasmas from optical measurements," Plasma Physics and Controlled Fusion, vol. 41 (9), pp. 1109-1123, 1999.

[42] A.J.A.J. Christlieb, W.N.G. Hitchon, J.E. Lawler, and G.G. Lister, “Integral and Lagrangian simulations of particle and radiation transport in plasma," Journal of Physics D: Applied Physics, vol. 42 (19), p. 194007, 2009.

[43] C.G. Mitchell and M.W. Zemansky, Resonance radiation and excited atoms vol. XVI. Cambridge: Cambridge University Press, 1971.

[44] D.J. Griffiths, "section 9.3: Spontaneous emission," in Introduction to quantum mechanics, 1st ed Upper Saddle River: Prentice Hall, 1995, p. 318.

[45] A.J. van Roosmalen, J.A.G. Baggerman, and S.J.H. Brader, Dry Etching for VLSI. New York: Plenum Press, 1991, pp. 40, 49, 62.

[46] T. Makabe and I. Petrovic, Plasma Electronics: Applications in Microelectronic Device Fabrication. Boca Raton: Taylor \& Francis, 2006, pp. 127-134.

[47] I.M. Podgornyi, Topics in Plasma Diagnostics. New York: Plenum Press, 1971, p. 39.

[48] T.I. Cox, V.G.I. Deshmukh, D.A.O. Hope, A.J. Hydes, N.S.J. Braithwaite, 
and N.M.P. Benjamin, "The use of Langmuir probes and optical emission spectroscopy to measure electron energy distribution functions in RFgenerated argon plasmas," Journal of Physics D: Applied Physics, vol. 20 (7), pp. 820-831, 1987.

[49] A.A. Shatas, Y.Z. Hu, and E.A. Irene, "Langmuir Probe and OpticalEmission Studies of $\mathrm{Ar}, \mathrm{O}_{2}$, and $\mathrm{N}_{2}$ Plasmas Produced by An ElectronCyclotron Resonance Microwave Source," Journal of Vacuum Science \& Technology A-Vacuum Surfaces and Films, vol. 10 (5), pp. 3119-3124, 1992.

[50] A. Hasper, J.E.J. Schmitz, J. Holleman, and J.F. Verwey, “Heat-Transport in Cold-Wall Single-Wafer Low-Pressure Chemical-Vapor-Deposition Reactors," Journal of Vacuum Science \& Technology A: Vacuum, Surfaces, and Films, vol. 10 (5), pp. 3193-3202, Sep-Oct 1992.

[51] A.M. van Graven and R.A.M. Wolters, "Wafer temperature measurement in PVD systems using the Co-Si reaction," Microelectronic Engineering, vol. 50 (1-4), pp. 495-499, 2000.

[52] I. Lin, D.C. Hinson, W.H. Class, and R.L. Sandstrom, "Low-energy high flux reactive ion etching by rf magnetron plasma," Applied Physics Letters, vol. 44 (2), pp. 185-187, 1984.

[53] H. Okano, T. Yamazaki, and Y. Horiike, "High rate reactive ion etching using a magnetron discharge," Solid State Technology, vol. 25 (April), pp. 166-170, 1982.

[54] A. Goede, G.J. Brakenhoff, H.J. Hopman, and P. Massmann, "Production of a Very Quiescent Plasma in "Skipping" Magnetic Fields," Physical Review Letters, vol. 27 (16), pp. 1044-1047, 1971.

[55] K.N. Leung, N. Hershkowitz, and K.R. MacKenzie, "Plasma confinement by localized cusps," Physics of Fluids, vol. 19 (7), pp. 1045-1053, 1976.

[56] K.N. Leung, T.K. Samec, and A. Lamm, "Optimization of permanent magnet plasma confinement," Physics Letters A, vol. 51 (8), pp. 490-492, 1975.

[57] M.A. Lieberman and A.J. Lichtenberg, "5.5 magnetic multipole confinement," in Principles of plasma discharges and materials processing 1st ed New York: John Wiley \& Sons, Inc., 1994, pp. 146-150.

[58] B. Andrieu, H. Beaujon, N. Launay, L. Popin, and M. Puech, "Personal communication at Alcatel Micro Machining Systems in Annecy," France, 2006-03-13.

[59] M.P.J. Tiggelman, "Thin film barium strontium titanate capacitors for tunable RF front-end applications," PhD. thesis, Twente University, Enschede, 2009, p. 163. 
[60] A.J. v. Roosmalen, J.A.G. Baggerman, and S.J.H. Brader, "Matching networks," in Dry Etching for VLSI, 1st ed New York: Plenum Press, 1991, pp. 77-79.

[61] J. Stefan, “Über die Beziehung zwischen der Wärmestrahlung und der Temperatur," Sitzungsberichte der Mathematischnaturwissenschaftlichen Classe der Kaiserlichen Akademie der Wissenschaften. Abt. 2, Mathematik, Physik, Chemie, Physiologie, Meteorologie, vol. 79 pp. 391-428, 1879.

[62] L. Boltzmann, “Ableitung des Stefan'schen Gesetzes, betreffend die Abhängigkeit der Wärmestrahlung von der Temperatur aus der electromagnetischen Lichttheorie," Annalen der Physik und Chemie, vol. 22 pp. 291294, 1884.

[63] M. Csele, Fundamentals of light sources and lasers. Hoboken: John Wiley \& Sons, 2004, pp. 2, 3.

[64] CRC handbook of chemistry and physics [Online]. Available: http://www.hbcpnetbase.com/.

[65] R.J. Visser, "Determination of the Power and Current Densities in Argon and Oxygen Plasmas by Insitu Temperature-Measurements," Journal of Vacuum Science \& Technology A-Vacuum Surfaces and Films, vol. 7 (2), pp. 189-194, 1989.

[66] S. Dushman and J.M. Lafferty, Scientific foundations of vacuum technique, 2nd rev. ed. New York: John Wiley \& Sons, 1966.

[67] S.C. Saxena and R.K. Joshi, Thermal Accommodation and Adsorbtion Coefficients of Gases vol. 2-1. New York: MacGraw-Hill, 1981.

[68] A. Ghatak, "Reflection and refraction of electromagnetic waves," in Optics, 1st ed Dubuque, IA [etc.]: McGraw-Hill, 2008, pp. 21-27.

[69] M. Born and E. Wolf, Principles of optics: electromagnetic theory of propagation, interference and diffraction of light, 6th ed. Oxford [etc.]: Pergamon, 1980.

[70] H.C.M. Ricken, Fysische chemie, deel 1A: Chemische thermodynamika, 3rd ed. Oss: Ricomp, 1991.

[71] M.A. Lieberman and A.J. Lichtenberg, "7.2 Energy and enthalpy," in Principles of plasma discharges and materials processing 1st ed New York: John Wiley \& Sons, Inc., 1994, pp. 192-197.

[72] F.F. Chen, "Langmuir probe analysis for high density plasmas," Physics of Plasmas, vol. 8 (6), pp. 3029-3041, 2001.

[73] E.H. Hirsch, "Plasma Probes and Langmuir Paradox," International Journal of Electronics, vol. 19 pp. 537-548, 1965.

[74] A.P. Paranjpe, J.P. McVittie, and S.A. Self, “A tuned Langmuir probe for 
measurements in rf glow discharges," Journal of Applied Physics, vol. 67 (11), pp. 6718-6727, 1990.

[75] V.A. Godyak, R.B. Piejak, and B.M. Alexandrovich, “Electron energy distribution function measurements and plasma parameters in inductively coupled argon plasma," Plasma Sources Science and Technology, vol. 11 (4), pp. 525-543, 2002.

[76] R.R.J. Gagné and A. Cantin, "Investigation of an rf Plasma with Symmetrical and Asymmetrical Electrostatic Probes," Journal of Applied Physics, vol. 43 (6), pp. 2639-2647, 1972.

[77] F.F. Chen, "Langmuir probes in RF plasma: surprising validity of OML theory," Plasma Sources Science and Technology, vol. 18 (3), p. 035012, 2009.

[78] S.C.M. Luijendijk and J. Van Eck, "Comparison of three devices for measuring the second derivative of a langmuir probe curve," Physica, vol. 36 (1), pp. 49-60, 1967.

[79] D. Herrmann and S. Klagge, “Untersuchungen zur Lage des Raumpotentials in der 2. Ableitung der Sondencharakteristik mit Hilfe von Glühsonden," Beiträge aus der Plasmaphysik, vol. 12 (1), pp. 17-25, 1972.

[80] M.B. Hopkins and W.G. Graham, "Langmuir probe technique for plasma parameter measurement in a medium density discharge," Review of Scientific Instruments, vol. 57 (9), pp. 2210-2217, 1986.

[81] J. Laframboise, in Rarefied gas dynamics. Proceedings of the fourth International Symposium, Toronto, 1966, p. 22.

[82] R.M. Clements, "Plasma diagnostics with electric probes," Journal of Vacuum Science and Technology, vol. 15 (2), pp. 193-198, 1978.

[83] V.A. Godyak and R.B. Piejak, "In situ simultaneous radio frequency discharge power measurements," Journal of Vacuum Science \& Technology A: Vacuum, Surfaces, and Films, vol. 8 (5), pp. 3833-3837, 1990.

[84] N. Kang, S.-g. Oh, and A. Ricard, "Determination of the electron temperature in a planar inductive argon plasma with emission spectroscopy and electrostatic probe," Journal of Physics D: Applied Physics, vol. 41 (15), p. 155203, 2008.

[85] N.C.M. Fuller, M.V. Malyshev, V.M. Donnelly, and I.P. Herman, “Characterization of transformer coupled oxygen plasmas by trace rare gasesoptical emission spectroscopy and Langmuir probe analysis," Plasma Sources, Science and Technology, vol. 9 (2), pp. 116-127, 2000.

[86] M.J. Druyvesteyn and F.M. Penning, "The Mechanism of Electrical Discharges in Gases of Low Pressure," Reviews of Modern Physics, vol. 12 (2), pp. 87-176, 1940. 
[87] M.J. Druyvesteyn and F.M. Penning, "Errata: Mechanism of Electrical Discharges in Gases of Low Pressure," Reviews of Modern Physics, vol. 13 (1), p. 72, 1941.

[88] V.A. Godyak, R.B. Piejak, and B.M. Alexandrovich, "Probe diagnostics of non-Maxwellian plasmas," Journal of Applied Physics, vol. 73 (8), pp. 3657-3663, 1993.

[89] M.J. Druyvesteyn, “Der Niedervoltbogen," Zeitschrift für Physik A Hadrons and Nuclei, vol. 64 (11, 12), pp. 781-798, 1930.

[90] D. Herrmann, S. Pfau, and H. Kagel, "Untersuchungen zur Frage des Plasmapotentials in der positiven Säule von Niederdruckentladungen," Beiträge aus der Plasmaphysik, vol. 12 (1), pp. 1-8, 1972.

[91] I. Langmuir and H. Mott-Smith, "Studies of Electric Discharges in Gases at Low Pressures, part I," General Electric Review, vol. 27 (7), pp. 449-455, 1924.

[92] I. Langmuir and H. Mott-Smith, "Studies of Electric Discharges in Gases at Low Pressures, part II," General Electric Review, vol. 27 (7), p. 538, 1924.

[93] I. Langmuir and H. Mott-Smith, "Studies of Electric Discharges in Gases at Low Pressures, part III," General Electric Review, vol. 27 (7), p. 616, 1924.

[94] I. Langmuir and H. Mott-Smith, "Studies of Electric Discharges in Gases at Low Pressures, part IV," General Electric Review, vol. 27 (7), p. 762, 1924.

[95] I. Langmuir and H. Mott-Smith, "Studies of Electric Discharges in Gases at Low Pressures, part V," General Electric Review, vol. 27 (7), p. 810, 1924.

[96] M.B. Hopkins, W.G. Graham, and T.J. Griffin, “Automatic Langmuir probe plasma diagnostic," Review of Scientific Instruments, vol. 58 (3), pp. 475-476, 1987.

[97] J.V. Scanlan and M.B. Hopkins, "Langmuir probe measurements of the electron energy distribution function in radio-frequency plasmas," Journal of Vacuum Science \& Technology A: Vacuum, Surfaces, and Films, vol. 10 (4), pp. 1207-1211, 1992.

[98] J. Richter, "Radiation of hot gases," in Plasma diagnostics, W. LochteHoltgreven, Ed., 1st ed Amsterdam: North-Holland publishing company, 1968, p. 3.

[99] E.U. Condon and G.H. Shortley, The theory of atomic spectra, 16th ed. Cambridge [etc.]: Cambridge University Press, 1999, pp. 188-191, 257264. 
[100] T. Makabe and I. Petrovic, Plasma Electronics: Applications in Microelectronic Device Fabrication. Boca Raton: Taylor \& Francis, 2006, p. 78.

[101] G.G. Raju, Gaseous electronics: Theory and practice. Boca Raton: Taylor \& Francis, 2006, p. 647.

[102] R.C. Elton, "Corona equilibrium model," in Methods of experimental physics, Plasma physics. vol. 9, part A, H.R. Griem and R.H. Lovberg, Eds., 1st ed London [etc.]: Academic Press, 1970, pp. 123, 124.

[103] H.R. Griem, "Corona-equilibrium relations," in Plasma spectroscopy, 1st ed New York [etc.]: McGraw-Hill, 1964, p. 159.

[104] H.R. Griem, "Boltzmann factors and Saha equations," in Plasma spectroscopy, 1st ed New York [etc.]: McGraw-Hill, 1964, p. 131.

[105] J.K. Ballou, C.C. Lin, and F.E. Fajen, "Electron-Impact Excitation of the Argon Atom," Physical Review A, vol. 8 (4), pp. 1797-1807, 1973.

[106] J.E. Chilton, J.B. Boffard, R.S. Schappe, and C.C. Lin, “Measurement of electron-impact excitation into the $3 p^{5} 4 p$ levels of argon using Fouriertransform spectroscopy," Physical Review A, vol. 57 (1), p. 267, 1998.

[107] S. Tsurubuchi, T. Miyazaki, and K. Motohashi, "Electron-impact emission cross sections of Ar," Journal of Physics B: Atomic, Molecular and Optical Physics, vol. 29 (9), pp. 1785-1801, 1996.

[108] T. Weber, J.B. Boffard, and C.C. Lin, "Electron-impact excitation cross sections of the higher argon $3 \mathrm{p}^{5} n \mathrm{p}(n=5,6,7)$ levels," Physical Review A, vol. 68 (3), p. 032719, 2003.

[109] H.R. Griem, Plasma spectroscopy. New York [etc.]: McGraw-Hill, 1964, p. 159.

[110] A.R. Filippelli, C.C. Lin, L.W. Anderson, and J.W. McConkey, "Principles and Methods for Measurement of Electron Impact Excitation Cross Sections for Atoms and Molecules by Optical Techniques," Advances in Atomic, Molecular, and Optical Physics, vol. 33 pp. 1-62, 1994.

[111] A. Chutjian and D.C. Cartwright, "Electron-impact excitation of electronic states in argon at incident energies between 16 and $100 \mathrm{eV}$," Physical Review A, vol. 23 (5), p. 2178, 1981.

[112] J.E. Chilton and C.C. Lin, "Measurement of electron-impact excitation into the $3 \mathrm{p}^{5} 3 \mathrm{~d}$ and $3 \mathrm{p}^{5} 5$ s levels of argon using Fourier-transform spectroscopy," Physical Review A, vol. 60 (5), pp. 3712-3721, 1999.

[113] J.B. Boffard, G.A. Piech, M.F. Gehrke, L.W. Anderson, and C.C. Lin, "Measurement of electron-impact excitation cross sections out of metastable levels of argon and comparison with ground-state excitation," Physical Review A, vol. 59 (4), p. 2749, 1999.

[114] G.A. Piech, J.B. Boffard, M.F. Gehrke, L.W. Anderson, and C.C. Lin, “Mea- 
surement of Cross Sections for Electron Excitation out of the Metastable Levels of Argon," Physical Review Letters, vol. 81 (2), pp. 309-312, 1998.

[115] H.M. Katsch and et al., "The influence of metastables on the plasmainduced emission in a helium rf discharge," Plasma Physics and Controlled Fusion, vol. 38 (2), pp. 183-192, 1996.

[116] N.K. Bibinov, D.B. Kokh, N.B. Kolokolov, V.A. Kostenko, D. Meyer, I.P. Vinogradov, and K. Wiesemann, "A comparative study of the electron distribution function in the positive columns in $\mathrm{N}_{2}$ and $\mathrm{N}_{2}$ / He dc glow discharges by optical spectroscopy and probes," Plasma Sources Science and Technology, vol. 7 (3), pp. 298-309, 1998.

[117] J.B. Boffard, C.C. Lin, and C.A.D. Jr, "Application of excitation cross sections to optical plasma diagnostics," Journal of Physics D: Applied Physics, vol. 37 (12), pp. R143-R161, 2004.

[118] J.B. Boffard, R.O. Jung, C.C. Lin, and A.E. Wendt, "Measurement of metastable and resonance level densities in rare-gas plasmas by optical emission spectroscopy," Plasma Sources Science and Technology, vol. 18 (3), p. 035017, 2009.

[119] I.P. Zapesochnyi, A.I. Imre, A.I. Dashchenko, V. Vukstich, S, F.F. Danch, and V.A. Kel'man, Soviet Physics: Journal of experimental and theoretical physics, vol. 36 pp. 1056-1060, 1973.

[120] N.C.M. Fuller, I.P. Herman, and V.M. Donnelly, “Optical actinometry of $\mathrm{Cl}_{2}, \mathrm{Cl}, \mathrm{Cl}^{+}$, and $\mathrm{Ar}^{+}$densities in inductively coupled $\mathrm{Cl}_{2}-\mathrm{Ar}$ plasmas," Journal of Applied Physics, vol. 90 (7), pp. 3182-3191, 2001.

[121] K. Behringer, "Diagnostics and modelling of ECRH microwave discharges," Plasma Physics and Controlled Fusion, vol. 33 (9), pp. 997-1028, 1991.

[122] R.L. Rhoades and S.M. Gorbatkin, "Characterization of Ar/Cu electroncyclotron-resonance plasmas using optical emission spectroscopy," Journal of Applied Physics, vol. 80 (5), pp. 2605-2613, 1996.

[123] J. Musielok, K.H. Finken, and U. Ackermann, "Simultaneous ionization and excitation of argon in an ionization wave," Physica B+C, vol. 125 (3), pp. 361-368, 1984.

[124] D.A.O. Hope, T.I. Cox, and V.G.I. Deshmukh, "Langmuir probe and optical emission spectroscopic studies of $\mathrm{Ar}$ and $\mathrm{O}_{2}$ plasmas," Vacuum, vol. $37(3,4)$, pp. 275-277, 1987.

[125] H. Suzuki and K. Nobata, "Measurements of hydrogen atom density in a Z-discharge plasma using intensities of $\mathrm{H} \alpha$ and $\mathrm{H} \beta$ lines," Japanese Journal of Applied Physics, Part 1 (Regular Papers \& Short Notes), vol. 25 (10), pp. 1589-1593, 1986. 
[126] T.M. Desai, S.V. Gogawale, A.B. Shukla, N.K. Joshi, H.S. Salgaonkar, and G.L. Bhale, "Electron temperature measurements in UHV systems by spectroscopic and Langmuir probe techniques," Vacuum, vol. 46 (3), pp. 223-226, 1995.

[127] J. Felts and E. Lopata, "Measurements of Electron-Temperature in A Capacitively Coupled Plasma Using Emission-Spectroscopy," Journal of Vacuum Science \& Technology A-Vacuum Surfaces and Films, vol. 6 (3), pp. 2051-2053, 1988.

[128] V.M. Donnelly, M.V. Malyshev, M. Schabel, A. Kornblit, W. Tai, I.P. Herman, and N.C.M. Fuller, "Optical plasma emission spectroscopy of etching plasmas used in Si-based semiconductor processing," Plasma Sources Science and Technology, vol. 11 (3A), pp. A26-A30, 2002.

[129] K.S. Fancey and A. Matthews, "Determination of electron temperature in ion plating discharges by optical emission spectroscopy," Vacuum, vol. 42 (15), pp. 1013-1015, 1991.

[130] V.M. Donnelly, "Plasma electron temperatures and electron energy distributions measured by trace rare gases optical emission spectroscopy," Journal of Physics D: Applied Physics, vol. 37 (19), pp. R217-R236, 2004.

[131] B.C. Smith, A. Khandelwal, and H.H. Lamb, "Ar/ $\mathrm{N}_{2} \mathrm{O}$ remote plasmaassisted oxidation of $\mathrm{Si}(100)$ : Plasma chemistry, growth kinetics, and interfacial reactions," Journal of Vacuum Science \& Technology B (Microelectronics and Nanometer Structures), vol. 18 (3), pp. 1757-1763, 2000.

[132] K.D. Bayes and G.B. Kistiakowsky, "On the Mechanism of the LewisRayleigh Nitrogen Afterglow," The Journal of Chemical Physics, vol. 32 (4), pp. 992-1000, 1960.

[133] S.E. Alexandrov and A.Y. Kovalgin, "Remote plasma-enhanced CVD of silicon nitride films: Effects of diluting nitrogen with argon. Part I: Effect on nitrogen plasma parameters studied by emission spectroscopy," Advanced Materials for Optics and Electronics, vol. 8 (1), pp. 13-22, 1998.

[134] T. Welzel, I. Dani, and F. Richter, "Determination of radical densities by optical emission spectroscopy during the ECR plasma deposition of SiC-N:H films using TMS as a precursor," Plasma Sources, Science and Technology, vol. 11 (3), pp. 351-359, 2002.

[135] N. Kosku, F. Kurisu, M. Takegoshi, H. Takahashi, and S. Miyazaki, “Highrate deposition of highly crystallized silicon films from inductively coupled plasma," Thin Solid Films, vol. 435 (1, 2), pp. 39-43, 2003.

[136] F. Perry, B. Stauder, G. Henrion, and P. Pigeat, "Plasma diagnostics for the control of reactive magnetron deposition process," Surface and Coatings Technology, vol. 74, 75 (Part 1), pp. 575-579, 1995. 
[137] A. Boogaard, A.Y. Kovalgin, A.A.I. Aarnink, R.A.M. Wolters, J. Holleman, I. Brunets, and J. Schmitz, "Langmuir-probe Characterization of an Inductively-Coupled Remote Plasma System intended for CVD and ALD," ECS Transactions, vol. 2 (7), pp. 181-191, 2007.

[138] A. Boogaard, A.Y. Kovalgin, I. Brunets, A.A.I. Aarnink, J. Holleman, R.A.M. Wolters, and J. Schmitz, "On the Verification of EEDFs in Plasmas with Silane using Optical Emission Spectroscopy," ECS Transactions, vol. 6 (1), pp. 259-270, 2007.

[139] J.A. Bittencourt, Fundamentals of plasma physics, 3rd ed. New york: Springer-Verlag, 2004, p. 168.

[140] A.D. MacDonald and S.J. Tetenbaum, in Gaseous Electronics vol. 1: electrical discharges, M.E. Hirsch and H.J. Oskam, Eds., 1st ed New York: Academic Press, 1978, p. 173.

[141] H.R. Koenig and L.I. Maissel, "Application of RF discharges to sputtering," IBM Journal of Research and Development, vol. 14 pp. 168-171, 1970.

[142] L.I. Maissel, in Handbook of Thin Film Technology, L.I. Maissel and R. Clang, Eds., 1st ed New York: McGraw-Hill, 1970, pp. 4-32.

[143] G.N. Jackson, “R.F. sputtering," Thin Solid Films, vol. 5 (4), pp. 209-246, 1970.

[144] V.A. Godyak and A.S. Khanneh, "Ion bombardment secondary electron maintenance of steady RF discharge," IEEE Transactions on Plasma Science, vol. 14 (2), pp. 112-123, 1986.

[145] O.A. Popov and V.A. Godyak, "Power dissipated in low-pressure radiofrequency discharge plasmas," Journal of Applied Physics, vol. 57 (1), pp. 53-8, 1985.

[146] J.H. Keller and W.B. Pennebaker, "Electrical properties of RF sputtering systems," IBM Journal of Research and Development, vol. 23 (1), pp. 3-15, 1979.

[147] G.G. Raju, Gaseous electronics: Theory and practice. Boca Raton: Taylor \& Francis, 2006, p. 97.

[148] B. Chapman, "Self-bias of rf electrodes," in Glow discharge processes, 1st ed New York [etc.]: Wiley-Interscience, 1980, pp. 143-146.

[149] J.B. Boffard, B. Chiaro, T. Weber, and C.C. Lin, "Electron-impact excitation of argon: Optical emission cross sections in the range of 300-2500 nm," Atomic Data and Nuclear Data Tables, vol. 93 (6), pp. 831-863, 2007.

[150] R.W.S. Pfau, "ELectron Collision Rates and Transport Coefficients of a Weakly Ionized dc Plasma in $\mathrm{ArSiH}_{1-4}$-Mixtures," Contributions to Plasma Physics, vol. 30 (5), pp. 587-597, 1990. 
[151] R. Nagpal and A. Garscadden, "Low energy electron collision cross sections for silane," Journal of Applied Physics, vol. 75 (2), pp. 703-709, 1994.

[152] M. Kurachi and Y. Nakamura, "Electron swarm parameters in $\mathrm{SiH}_{4}$-rare gas mixtures and collision cross sections for monosilane molecules," IEEE Transactions on Plasma Science, vol. 19 (2), pp. 262-269, 1991.

[153] V.M. Donnelly and M.J. Schabel, "Spatially resolved electron temperatures, species concentrations, and electron energy distributions in inductively coupled chlorine plasmas, measured by trace-rare gases optical emission spectroscopy," Journal of Applied Physics, vol. 91 (10), pp. 6288-6295, 2002.

[154] A.Y. Kovalgin, A. Boogaard, I. Brunets, J. Holleman, and J. Schmitz, "Chemical modeling of a high-density inductively-coupled plasma reactor containing silane," Surface and Coatings Technology, vol. 201 (22, 23), pp. 8849-8853, 2007.

[155] A. Kovalgin, A. Boogaard, and R. Wolters, "Impact of Small Deviations in EEDF on Silane-based Plasma Chemistry," ECS Transactions, vol. 25 (8), pp. 429-436, 2009.

[156] A. Kovalgin, A. Boogaard, I. Brunets, T. Aarnink, and R. Wolters, "Electrical Properties of Plasma-Deposited Silicon Oxide Clarified by Chemical Modeling," ECS Transactions, vol. 25 (8), pp. 23-32, 2009.

[157] R.J. Buss, P. Ho, and M.E. Weber, "Laser studies of the reactivity of SiO with the surface of a depositing film," Plasma Chemistry and Plasma Processing, vol. 13 (1), pp. 61-76, 1993.

[158] J. Perrin, M. Shiratani, P. Kae-Nune, H. Videlot, J. Jolly, and J. Guillon, "Surface reaction probabilities and kinetics of $\mathrm{H}, \mathrm{SiH}_{3}, \mathrm{Si} 2 \mathrm{H} 5, \mathrm{CH} 3$, and C2H5 during deposition of a-Si:H and a-C:H from $\mathrm{H}_{2}, \mathrm{SiH}_{4}$, and $\mathrm{CH} 4$ discharges," Jounal of Vacuum Science and Technology A, vol. 16 (1), pp. 278-289, 1998.

[159] J. Robertson, "Deposition mechanism of hydrogenated amorphous silicon," Journal of Applied Physics, vol. 87 (5), pp. 2608-2617, 2000.

[160] R. Dewarrat and J. Robertson, "Binding and surface diffusion of $\mathrm{SiH}_{3}$ radicals on a growing a-Si:H surface," Journal of Non-Crystalline Solids, vol. 299-302 (Part 1), pp. 48-52, 2002.

[161] D.A. Doughty, J.R. Doyle, G.H. Lin, and A. Gallagher, "Surface reaction probability of film-producing radicals in silane glow discharges," Journal of Applied Physics, vol. 67 (10), pp. 6220-6228, 1990.

[162] M.D. Allendorf and R.J. Kee, "A Model of Silicon Carbide Chemical Va- 
por Deposition," Journal of The Electrochemical Society, vol. 138 (3), pp. 841-852, 1991.

[163] L.D. Thanh and P. Balk, "Elimination and Generation of $\mathrm{Si}_{-} \mathrm{SiO}_{2}$ Interface Traps by Low Temperature Hydrogen Annealing," Journal of The Electrochemical Society, vol. 135 (7), pp. 1797-1801, 1988.

[164] M.L. Reed and J.D. Plummer, "Chemistry of $\mathrm{Si}_{-} \mathrm{SiO}_{2}$ interface trap annealing," Journal of Applied Physics, vol. 63 (12), pp. 5776-5793, 1988.

[165] K. Sinniah, M.G. Sherman, L.B. Lewis, W.H. Weinberg, J.J.T. Yates, and K.C. Janda, "Hydrogen desorption from the monohydride phase on Si(100)," The Journal of Chemical Physics, vol. 92 (9), pp. 5700-5711, 1990.

[166] D.D. Koleske, S.M. Gates, and B. Jackson, "Atomic H abstraction of surface H on Si: An Eley-Rideal mechanism?," The Journal of Chemical Physics, vol. 101 (4), pp. 3301-3309, 1994.

[167] C.M. Chiang, S.M. Gates, S.S. Lee, M. Kong, and S.F. Bent, “Etching, Insertion, and Abstraction Reactions of Atomic Deuterium with Amorphous Silicon Hydride Films," The Journal of Physical Chemistry B, vol. 101 (46), pp. 9537-9547, 1997.

[168] G.V. Bianco, M. Losurdo, M.M. Giangregorio, P. Capezzuto, and G. Bruno, "Real time monitoring of the interaction of $\mathrm{Si}(100)$ with atomic hydrogen: The "h-insertion/Si-etching" kinetic model explaining Si surface modifications," Applied Physics Letters, vol. 95 (16), p. 161501, 2009.

[169] M. Ritala and M. Leskelä, "Atomic layer deposition," in Deposition and processing of thin films. vol. 1, N. Hari Singh, Ed., 1st ed Burlington: Academic Press, 2002, pp. 103-159.

[170] V.P. Tolstoy, I.V. Chernyshova, and V.A. Skryshevsky, "6.6.1. Monitoring of $\mathrm{CVD}$ of $\mathrm{SiO}_{2}$," in Handbook of infrared spectroscopy of ultrathin films, 1st ed Hoboken: John Wiley \& Sons, Inc., 2003, pp. 502-504.

[171] H.G. Tompkins and W.A. McGahan, Spectroscopic Ellipsometry and Reflectometry: A User's Guide. New York [etc.]: John Wiley \& Sons, Inc., 1999.

[172] L.X. Yi and et al., "Phase separation of thin SiO layers in amorphous $\mathrm{SiO} / \mathrm{SiO}_{2}$ superlattices during annealing," Journal of Physics: Condensed Matter, vol. 15 (39), pp. S2887-S2895, 2003.

[173] H.J. Fitting, T. Barfels, A.N. Trukhin, B. Schmidt, A. Gulans, and A. von Czarnowski, "Cathodoluminescence of $\mathrm{Ge}^{+}, \mathrm{Si}^{+}$, and $\mathrm{O}^{+}$implanted $\mathrm{SiO}_{2}$ layers and the role of mobile oxygen in defect transformations," Journal of Non-Crystalline Solids, vol. 303 (2), pp. 218-231, 2002.

[174] J.J.H. Gielis, B. Hoex, M.C.M. v.d. Sanden, and W.M.M. Kessels, “Negative charge and charging dynamics in $\mathrm{Al}_{2} \mathrm{O}_{3}$ films on Si characterized by 
second-harmonic generation," Journal of Applied Physics, vol. 104 (7), p. 073701, 2008.

[175] G. Lüpke, "Characterization of semiconductor interfaces by secondharmonic generation," Surface Science Reports, vol. 35 (3, 4), pp. 75-161, 1999.

[176] A. Stesmans and V.V. Afanas'ev, "Electrical activity of interfacial paramagnetic defects in thermal (100) $\mathrm{Si} / \mathrm{SiO}_{2}$," Physical Review B, vol. 57 (16), pp. 10030-10034, 1998.

[177] V.P. Tolstoy, I.V. Chernyshova, and V.A. Skryshevsky, "6.5 IR-spectroscopy of surface states at $\mathrm{SiO}_{2}-\mathrm{Si}$ interface," in Handbook of infrared spectroscopy of ultrathin films, 1st ed Hoboken: John Wiley \& Sons, Inc., 2003, pp. 497-501.

[178] V.V. Afanas'ev, Internal Photoemission Spectroscopy, Principles and Applications, 1st ed. Oxford [etc.]: Elsevier Ltd., 2008.

[179] H.J. Fitting, P. Magdanz, W. Mehnert, D. Hecht, and T. Hingst, "Charge Trap Spectroscopy in Single and Multiple Layer Dielectrics," Physica Status Solidi a - Applied Research, vol. 122 (1), pp. 297-309, Nov 1990.

[180] R. Ludeke and E. Cartier, "Imaging of trapped charge in $\mathrm{SiO}_{2}$ and at the $\mathrm{SiO}_{2}-\mathrm{Si}$ interface," Applied Physics Letters, vol. 78 (25), pp. 3998-4000, 2001.

[181] S.M. Sze and K.K. Ng, "Chapter 4. Metal-insulator-semiconductor capacitors," in Physiscs of Semiconductor Devices, third edition ed New York: Wiley-Interscience, 2007, pp. 197-238.

[182] D.K. Schroder, "6.2 Fixed, oxide trapped, and mobile oxide charge," in Semiconductor material and device characterization, 3rd ed Hoboken: John Wiley and Sons, Inc., 2006, pp. 321-342.

[183] J.S. Kang and D.K. Schroder, "The Pulsed Mis Capacitor - a CriticalReview," Physica Status Solidi a - Applied Research, vol. 89 (1), pp. 13-43, 1985.

[184] D.K. Schroder, Semiconductor material and device characterization, 3rd ed. Hoboken: John Wiley and Sons, Inc., 2006.

[185] I. Brunets, "Electronic devices fabricated at CMOS backend-compatible temperatures," Ph.D. thesis, Universiteit Twente, Enschede, 2009.

[186] P.E. Nicollian, "Effect of interface traps and oxide fixed charge," Ph.D. thesis, Physics of Trap Generation and Electrical Breakdown in Ultra-thin $\mathrm{SiO}_{2}$ and $\mathrm{SiON}$ Gate Dielectric Materials, University of Twente, Enschede, 2007, pp. 23, 24.

[187] R. Castagné and A. Vapaille, “Description of the $\mathrm{SiO}_{2}-\mathrm{Si}$ interface prop- 
erties by means of very low frequency MOS capacitance measurements," Surface Science, vol. 28 (1), pp. 157-193, 1971.

[188] S. Bystrova, "Diffusion barriers for Cu metallisation in Si integrated circuits: deposition and related thin film properties," Ph.D. thesis, Enschede, 2004, p. 28.

[189] A. Boogaard, A.Y. Kovalgin, I. Brunets, A.A.I. Aarnink, J. Holleman, R.A.M. Wolters, and J. Schmitz, "Characterization of $\mathrm{SiO}_{2}$ films deposited at low temperature by means of remote ICPECVD," Surface and Coatings Technology, vol. 201 (22, 23), pp. 8976-8980, 2007.

[190] R.F. Pierret, "MOS fundamentals," in Field-effect devices. vol. IV, 2nd ed Amsterdam [etc.]: Addison-Wesley, 1996, pp. 33-40.

[191] R.F. Pierret, "Appendix B. Semiconductor electrostatics — Exact solution," in Field-effect devices. vol. IV, 2nd ed Amsterdam [etc.]: AddisonWesley, 1996, pp. 165-168.

[192] B.L. Yang, H. Wong, and Y.C. Cheng, "Study of process-dependent electron-trapping characteristics of thin nitrided oxides," Solid-State Electronics, vol. 37 (3), pp. 481-486, 1994.

[193] D.J. DiMaria, R. Ghez, and D.W. Dong, "Charge trapping studies in $\mathrm{SiO}_{2}$ using high current injection from Si-rich $\mathrm{SiO}_{2}$ films," Journal of Applied Physics, vol. 51 (9), pp. 4830-4841, 1980.

[194] S.M. Sze, Physics of Semiconductor devices, 2nd ed. New York: WileyInterscience, 1981, p. 397.

[195] Z. Wang, "Detection of and protection against plasma charging damage in modern ic technology," Ph.D. thesis, Enschede, 2004, p. 131.

[196] J.G.G. Ackaert, "Dielectric engineering: characterization, development and proces damage minimization of various silicon oxides," Ph.D. thesis, Enschede, 2004.

[197] I. Brunets, A. Boogaard, S. Smits, H. De Vries, A.A.I. Aarnink, J. Holleman, A. Kovalgin, and J. Schmitz, "Low temperature silicon TFTs with poly-stripes," in International Thin Film Transistor Conference (ITC'09), Palaiseau, France, 2009, pp. 62-65.

[198] I. Brunets, J. Holleman, A.Y. Kovalgin, A. Boogaard, and J. Schmitz, "Low-Temperature Fabricated TFTs on Polysilicon Stripes," IEEE Transactions on Electron Devices, vol. 56 (8), pp. 1637-1644, Aug 2009.

[199] I. Brunets, J. Holleman, A.Y. Kovalgin, and J. Schmitz, "Poly-Si stripe TFTs by grain-boundary controlled crystallization of amorphous-Si," in 38th European Solid-State Device Research Conference (ESSDERC 2008), Edinburgh, Schotland, 2008, pp. 87-90.

[200] S.-I. Saito, K. Torii, Y. Shimamoto, O. Tonomura, D. Hisamoto, T. Onai, 
M. Hiratani, S. Kimura, Y. Manabe, M. Caymax, and J.W. Maes, “Remotecharge-scattering limited mobility in field-effect transistors with $\mathrm{SiO}_{2}$ and $\mathrm{Al}_{2} \mathrm{O}_{3} / \mathrm{SiO}_{2}$ gate stacks," Journal of Applied Physics, vol. 98 (11), pp. 113706-113709, 2005.

[201] L. Faraone, R.D. Vibronek, and J.T. McGinn, "Characterization of thermally oxidized n+ polycrystalline silicon," IEEE Transactions on Electron Devices, vol. 32 (3), pp. 577-583, 1985.

[202] L. Faraone, "Thermal $\mathrm{SiO}_{2}$ films on $\mathrm{n}+$ polycrystalline silicon: Electrical conduction and breakdown," IEEE Transactions on Electron Devices, vol. 33 (11), pp. 1785-1794, 1986.

[203] S.L. Wu, C.Y. Chen, T.Y. Lin, C.L. Lee, T.F. Lei, and M.S. Liang, "Polyoxides grown on n+ polysilicon," IEEE Transactions on Electron Devices, vol. 44 (1), pp. 153-159, 1997.

[204] B. Hoex, J.J.H. Gielis, M.C.M. v.d. Sanden, and W.M.M. Kessels, “On the c-Si surface passivation mechanism by the negative-charge-dielectric $\mathrm{Al}_{2} \mathrm{O}_{3}$," Journal of Applied Physics, vol. 104 (11), p. 113703, 2008.

[205] A. Boogaard, A.Y. Kovalgin, and R.A.M. Wolters, "Net negative charge in low-temperature $\mathrm{SiO}_{2}$ gate dielectric layers," Microelectronic Engineering, vol. 86 (7-9), pp. 1707-1710, 2009.

[206] R.F. Pierret, "18.2 Oxide Charges " in Semiconductor Device Fundamentals, ed Amsterdam: Addison Wesley Longman, 1996, p. 649.

[207] A.S. Grove, "12.4 Barrier energies," in Physics and technology of semiconductor devices, ed New York: John Wiley and Sons, Inc., 1967, pp. $345,346$.

[208] S.S. Iyer, P.M. Solomon, V.P. Kesan, A.A. Bright, J.L. Freeouf, T.N. Nguyen, and A.C. Warren, "A gate-quality dielectric system for SiGe metal-oxidesemiconductor devices," Electron Device Letters, IEEE, vol. 12 (5), pp. 246-248, 1991.

[209] A.Y. Kovalgin, G. Isai, J. Holleman, and J. Schmitz, "Low-Temperature $\mathrm{SiO}_{2}$ Layers Deposited by Combination of ECR Plasma and Supersonic Silane/Helium Jet," Journal of The Electrochemical Society, vol. 155 (2), pp. G21-G28, 2008.

[210] M.J. Hernandez, J. Garrido, J. Martinez, and J. Piqueras, “Electrical properties of electron cyclotron resonance plasma-deposited silicon dioxide: effect of the oxygen to silane flow ratio," Semiconductor Science and Technology, vol. 11 (3), pp. 422-426, 1996.

[211] T. Yasuda, Y. Ma, S. Habermehl, and G. Lucovsky, "Low-temperature formation of device-quality $\mathrm{SiO}_{2} / \mathrm{Si}(100)$ interfaces by a two-step remote 
plasma-assisted oxidation/deposition process," Applied Physics Letters, vol. 60 (4), p. 434, 1992.

[212] M. Tabakomori and H. Ikoma, "Low-Temperature Si Oxidation Using Inductively Coupled Oxygen-Argon Mixed Plasma," Japanese Journal of Applied Physics Part 1-Regular Papers Short Notes \& Review Papers, vol. 36 (9A), pp. 5409-5415, 1997.

[213] J. Robertson, "Band offsets of high dielectric constant gate oxides on silicon," Journal of Non-Crystalline Solids, vol. 303 (1), pp. 94-100, 2002.

[214] R.B. Beck, "Formation of ultrathin silicon oxides-modeling and technological constraints," Materials Science in Semiconductor Processing, vol. 6 (1-3), pp. 49-57, 2003.

[215] C.R. Helm and B.E. Deal, Eds., The Physics and Chemistry of $\mathrm{SiO}_{2}$ and the $\mathrm{Si}_{-} \mathrm{SiO}_{2}$ Interface. New York: Plenum, 1993.

[216] V.V. Afanas'ev and A. Stesmans, "H-complexed oxygen vacancy in $\mathrm{SiO}_{2}$ : Energy level of a negatively charged state," Applied Physics Letters, vol. 71 (26), pp. 3844-3846, 1997.

[217] C.S. Ewig and J. Tellinghuisen, "Ab initio study of the electronic states of $\mathrm{O}_{2}^{-}$in vacuo and in simulated ionic solids," The Journal of Chemical Physics, vol. 95 (2), pp. 1097-1106, 1991.

[218] H.J. Fitting, R. Salh, T. Barfels, and B. Schmidt, "Multimodal luminescence spectra of ion-implanted silica," physica status solidi (a), vol. 202 (13), pp. R142-R144, 2005.

[219] R. Salh, A. von Czarnowski, and H.J. Fitting, "Cathodoluminescence of non-stoichiometric silica: The role of oxygen," Journal of Non-Crystalline Solids, vol. 353 (5-7), pp. 546-549, 2007.

[220] J. Lindhard and M. Scharff, "Energy Dissipation by Ions in the kev Region," Physical Review, vol. 124 (1), pp. 128-130, 1961.

[221] N. Mott, "Mechanisms for the Oxidation of Silicon and the Formation of Charged Defects," Proceedings of the Royal Society of London. Series A, Mathematical and Physical Sciences, vol. 376 (1765), pp. 207-215, 1981.

[222] J. Appels and H.M.J. Vaes, "High voltage thin layer devices (RESURF devices)," in IEEE Int. Electron Devices Meeting, 1979, pp. 238-241. 


\section{Summary}

The fabrication of electronic devices at relatively low temperatures $\left(100-150^{\circ} \mathrm{C}\right)$ is becoming increasingly important. It is possible to fabricate devices on glass or polymer substrates at low temperatures, or one can add extra functionality to standard ICs through post-processing. In order to realize good devices, we need to fabricate high-electrical quality dielectrics and semiconductors, which is a challenge at such low temperatures. Plasma-enhanced chemical vapor deposition allows $\mathrm{SiO}_{2}$ films to deposit at temperatures of around $400^{\circ} \mathrm{C}$ and lower. It is an experimental fact, however, that a low deposition temperature results in a degradation of the film properties. This degradation can be minimized partly by using high-density remote plasma (HDRP) reactors, as these reactors are capable of operating at lower pressures, thereby reducing gas-phase nucleation. They can also maintain higher plasma densities, thus enabling a higher rate of electron-stimulated reactions.

Our first objective was to contribute to PECVD technology by exploring its capability to fabricate high-quality silicon dioxide gate dielectrics at low substrate temperatures. Our second objective was to contribute to a more exact understanding of PECVD technology by demonstrating that an accurate description of plasma parameters and chemistry can help researchers find optimal process conditions.

We built a remote ICPECVD system to deposit thin $\mathrm{SiO}_{2}$ dielectric films. The plasma was determined using a Langmuir probe and optical emission spectroscopy. Furthermore, we started modeling chemical reactions in $\mathrm{Ar}-\mathrm{N}_{2} \mathrm{O}-$ 
$\mathrm{SiH}_{4}$ plasmas in our search for optimum process conditions in our ICP reactor. Electron density and electron energy distribution function (EEDF) were measured using a Langmuir probe. The EEDF of Ar plasma in the reactor could largely be described by the Maxwell-Boltzmann distribution function, but it also contained a fraction $\left(\sim 10^{-3}\right)$ of electrons which were much faster $(20-50$ $\mathrm{eV})$. We demonstrated that ignoring this tail would result in significant modeling and interpretation errors.

Because of the Langmuir probe method's limitations, these experimental EEDFs could only be obtained without silane in the plasma reactor. To overcome these limitations, we developed a method to obtain EEDFs for depositing plasmas with silane using a combination of the Langmuir probe method and optical emission spectroscopy (OES). We demonstrated that EEDFs in our reactor, as measured by the Langmuir probe in $\mathrm{Ar}-\mathrm{N}_{2}$ and $\mathrm{Ar}-\mathrm{N}_{2} \mathrm{O}$ plasmas, resemble EEDFs in plasmas with small additions of silane, provided that silane fractions in the plasma are below $2 \%$ at total pressures of between 1-6 Pa. As such, the EEDF measured without silane can be used as input for chemical modeling and optimizing deposition processes in plasmas containing silane.

We modeled the composition of an $\mathrm{Ar}-\mathrm{SiH}_{4}-\mathrm{N}_{2} \mathrm{O}$ ICPECVD system. We used both the experimental and MB EEDFs to calculate reaction rate constants for the electron impact reactions. We demonstrated that the use of an inappropriate EEDF, which at first sight only deviates slightly from the experimental distribution, could cause strong disagreements between the actual plasma composition and the model. We then used our simulations to predict qualitatively (in terms of concentrations of the reactive species) the influence of the $\mathrm{SiH}_{4} / \mathrm{N}_{2} \mathrm{O}$ gas-flow ratio and total gas pressure on the film's electrical properties and deposition rate.

We deposited silicon dioxide films in plasma conditions similar to those in the model. It appeared that the predictions we made through the model could be verified in the experiment. As such, we proved the combination of electrical measurements, plasma characterization and plasma modeling to be valuable tools in ICP reactor process control and optimization.

The optimization led to the deposition of films with high dielectric quality at a deposition temperature of only $150^{\circ} \mathrm{C}$. The films exhibited very low leakage currents at electric field strength of $6.5 \mathrm{MV} / \mathrm{cm}$, which was comparable to the leakage currents of thermally grown oxides. We obtained a very low midgap- $D_{\text {it }}$ of $3 \cdot 10^{10} \mathrm{eV}^{-1} \mathrm{~cm}^{-2}$. We showed the applicability of our silicon dioxide films to low-temperature TFTs by demonstrating their competitive mobility values and low off-currents. The described low-temperature PECVD oxide can also be developed for low-temperature CMOS post-processing. This 
CMOS process can be employed for large-area TFTs as well as in 3-D electronics due to its low thermal budget.

The net charge of our ICPECVD oxides appeared to be negative and a function of layer thickness. We demonstrated that two mechanisms contributed to the film growth and charge formation, namely plasma oxidation of the silicon substrate and chemical vapor deposition. We suggest that the first nm-range of oxide thickness is formed by plasma oxidation that added a negative charge to the interfacial oxide layer, while the CVD component added a positive charge to the bulk oxide.

We conclude that measuring actual plasma parameters in combination with an accurate description of plasma chemistry can help researchers reach optimal conditions for the process. In this way researchers are not left with the sole approach of trial and error. The described method enables the fabrication of high-quality silicon dioxide gate dielectrics using ICPECVD technology. 


\section{Samenvatting}

De productie van elektronische schakelingen bij relatief lage temperaturen (100$150^{\circ} \mathrm{C}$ ) wordt steeds belangrijker. Het is mogelijk om bij lage temperaturen schakelingen te produceren op substraten van glas of polymeer, of om achteraf extra functionaliteit toe te voegen aan standaard IC's. Om goede schakelingen te verkrijgen dien je diëlektrica en halfgeleiders te produceren met goede elektrische eigenschappen, wat niet gemakkelijk is bij zulke lage temperaturen. Met PECVD (door plasma versterkte chemische dampdepositie) is depositie mogelijk van $\mathrm{SiO}_{2}$ lagen bij temperaturen van $400^{\circ} \mathrm{C}$ of lager. Bij onderzoek is echter vastgesteld dat een lage depositietemperatuur resulteert in degradatie van de filmeigenschappen. Deze degradatie kan deels geminimaliseerd worden door HDRP-reactoren (hoge-dichtheid remote plasma) te gebruiken, aangezien die bij een lagere druk kunnen functioneren waardoor kiemvorming in de gasfase afneemt. HDRP-reactoren kunnen ook een hogere plasmadichtheid behouden en daardoor een groter aantal door elektronen gestimuleerde reacties mogelijk maken.

Ons eerste doel was om bij te dragen aan de PECVD-technologie door middel van onderzoek naar haar vermogen om hoge-kwaliteit siliciumdioxide voor metaal-oxide-silicium (MOS) toepassingen te produceren bij lage substraattemperaturen. Ons tweede doel was om bij te dragen aan een nauwkeuriger inzicht van de PECVD-technologie door te demonstreren dat een juiste beschrijving van plasmaparameters en plasmachemie onderzoekers kan bijstaan bij het vinden van optimale procesomstandigheden. 
We hebben een remote ICPECVD-systeem gebouwd voor depositie van dunne $\mathrm{SiO}_{2}$ diëlektrische lagen. De eigenschappen van het plasma werden vastgesteld met een Langmuirsonde en met optische emissiespectroscopie. Verder modelleerden we chemische reacties in $\mathrm{Ar}-\mathrm{N}_{2} \mathrm{O}-\mathrm{SiH}_{4}$ plasma's in onze zoektocht naar optimale procesomstandigheden in onze ICP-reactor.

De elektronendichtheid en de snelheidsverdelingsfunctie van de elektronen (EEDF) werden gemeten met een Langmuir-sonde. De EEDF van Arplasma in de reactor kon grotendeels worden beschreven met de MaxwellBoltzmann verdelingsfunctie, maar het bevatte ook een weinig $\left(\sim 10^{-3}\right)$ elektronen die veel sneller waren $(20-50 \mathrm{eV})$. We hebben laten zien dat het negeren van die snelle elektronen resulteert in aanzienlijke modelleer- en interpretatiefouten.

Vanwege de beperkingen van de Langmuir-sonde konden deze experimentele EEDF's alleen worden bepaald zonder silaan in de plasmareactor. Om deze beperkingen te kunnen overwinnen, hebben we een methode ontwikkeld om EEDF's te verkrijgen voor depositieplasma's met silaan met behulp van een combinatie van de Langmuir-sondemethode en optische emissiespectroscopie (OES). We hebben laten zien dat de EEDF's in onze reactor, gemeten met de Langmuir-sonde in Ar- $\mathrm{N}_{2}$ en $\mathrm{Ar}-\mathrm{N}_{2} \mathrm{O}$ plasma's, lijken op EEDF's in plasma's met kleine hoeveelheden silaan, ervan uitgaande dat de silaanhoeveelheden in het plasma minder zijn dan $0.8 \%$ bij een totale druk van tussen de 1 en 6 $\mathrm{Pa}$. Zo kan de EEDF gemeten zonder silaan gebruikt worden als uitgangspunt voor chemisch modelleren en om het depositieproces in plasma's met silaan te optimaliseren.

Wij modelleerden de samenstelling van een $\mathrm{Ar}-\mathrm{SiH}_{4}-\mathrm{N}_{2} \mathrm{O}$ ICPECVDsysteem. We gebruikten zowel de experimentele EEDF's als die van de MB om de reactieconstantes te berekenen voor de botsingsreacties van de elektronen. We hebben laten zien dat gebruik van een misplaatste EEDF, die in eerste instantie slechts enigszins afwijkt van de experimentele verdeling, sterke afwijkingen kan veroorzaken tussen de eigenlijke plasmasamenstelling en het model. De simulaties hebben we gebruikt om een kwantitatieve voorspelling te doen van de invloed van de $\mathrm{SiH}_{4} / \mathrm{N}_{2} \mathrm{O}$ gasstroomverhouding en totale gasdruk op de elektrische eigenschappen en depositiesnelheid van de laag.

We deponeerden siliciumdioxidelagen bij plasmaomstandigheden vergelijkbaar met die van het model. Onze voorspellingen met het model konden we experimenteel verifiëren. Zo hebben we bewezen dat de combinatie van elektrische metingen, plasmakarakterisering en plasmamodellering waardevolle instrumenten zijn voor processturing en procesoptimalisatie bij ICP-reactors.

De optimalisering leidde tot depositie van lagen met goede diëlektrische 
eigenschappen bij een depositietemperatuur van slechts $150^{\circ} \mathrm{C}$. De lagen vertoonden heel lage lekstromen bij een elektrische veldsterkte van $6,5 \mathrm{MV} / \mathrm{cm}$, wat vergelijkbaar is met de lekstromen bij thermisch gegroeide oxides. We verkregen een heel lage midgap- $D_{\text {it }}$ van $3 \cdot 10^{10} \mathrm{eV}^{-1} \mathrm{~cm}^{-2}$. We hebben de toepasbaarheid van onze siliciumdioxidelagen bij lage-temperatuur gemaakte dunne-laag-transistoren (TFT's) aangetoond door hun concurrerende mobiliteitswaarden en lage lekstromen te demonstreren. De beschreven lage-temperatuur PECVD-oxide zou ook ontwikkeld kunnen worden voor bij lage temperatuur geproduceerde CMOS-schakelingen. Dit CMOS-proces kan gebruikt worden voor een groot deel van de TFT- en 3D-elektronica dankzij de lage substraattemperatuur tijdens fabricage.

De netto lading van onze ICPECVD-oxides bleek negatief en bovendien een functie van de laagdikte te zijn. We hebben gedemonstreerd dat twee mechanismen bijdragen aan de opbouw van de film en de ladingvorming, namelijk plasmaoxidatie van het siliciumsubstraat en chemische dampdepositie. Onze resultaten wezen erop dat de eerste paar nanometers van de oxidedikte wordt gevormd door plasmaoxidatie die een negatieve lading toevoegt aan het grenslaagoxide, terwijl de CVD-component een positieve lading toevoegt aan het bulkoxide.

We concluderen dat onderzoekers gebaat zijn bij het meten van de feitelijke plasmaparameters in combinatie met een juiste beschrijving van de plasmachemie om optimale procesomstandigheden te bereiken. Onderzoekers hoeven dat nu dus niet meer alleen proefondervindelijk vast te stellen. De beschreven methode maakt het mogelijk om hoge-kwaliteits siliciumdioxide in MOS-structuren te fabriceren door middel van ICPECVD-technologie. 
Arjen Boogaard was born on September 10th, 1971 in Koudekerke. He received the Bachelor of Applied Sciences degree from Zeeland Polytechnic, Vlissingen. He joined Philips Research in 1996 where he co-developed a portable electrostatic air cleaner and studied the adhesion of polymers and phosphors on TV screens. He then became involved in thin film technology. He contributed to the development of a transceiver IC for cellular telephones, which was successfully transferred to a production facility. He also worked on electron beam lithography and on ASML lithography systems. He currently holds eight US patents. From 2004 to 2009 he was employed by the MESA+ Institute for Nanotechnology, chair of Semiconductor Components, University of Twente. This thesis is the result of research carried out during that period. Currently, he is employed at ASML. Arjen Boogaard is a Senior Member of the IEEE. 
[1] B. Rajasekharan, C. Salm, R.A.M. Wolters, A.A.I. Aarnink, A. Boogaard, and J. Schmitz, "Metal contacts to lowly doped Si and ultra thin SOI," in Proceedings of Fifth Workshop of the Thematic Network on Silicon on Insulator Technology, Devices and Circuits, 19-21 Jan 2009, Gotheburg, Sweden, 2009, pp. 29, 30.

[2] A. Kovalgin, A. Boogaard, and R. Wolters, "Impact of Small Deviations in EEDF on Silane-based Plasma Chemistry," ECS Transactions, vol. 25 (8), pp. 429-436, 2009.

[3] A. Kovalgin, A. Boogaard, I. Brunets, T. Aarnink, and R. Wolters, "Electrical Properties of Plasma-Deposited Silicon Oxide Clarified by Chemical Modeling," ECS Transactions, vol. 25 (8), pp. 23-32, 2009.

[4] I. Brunets, J. Holleman, A.Y. Kovalgin, A. Boogaard, and J. Schmitz, "LowTemperature Fabricated TFTs on Polysilicon Stripes," IEEE Transactions on Electron Devices, vol. 56 (8), pp. 1637-1644, Aug 2009.

[5] I. Brunets, A. Boogaard, S. Smits, H. De Vries, A.A.I. Aarnink, J. Holleman, A. Kovalgin, and J. Schmitz, "Low temperature silicon TFTs with poly-stripes," in International Thin Film Transistor Conference (ITC'09), Palaiseau, France, 2009, pp. 62-65.

[6] A. Boogaard, A.Y. Kovalgin, and R.A.M. Wolters, "Net negative charge in low-temperature $\mathrm{SiO}_{2}$ gate dielectric layers," Microelectronic Engineering, vol. 86 (7-9), pp. 1707-1710, 2009.

[7] I. Brunets, A.W. Groenland, A. Boogaard, A.A.I. Aarnink, and A.Y. 
Kovalgin, "A study of thermal oxidation and plasma-enhanced oxidation/reduction of ALD TiN layers," in Proceedings of the 18th International Conference on Atomical Layer Deposition ALD, Bruges, Belgium, 2008, pp. P-54.

[8] A.Y. Kovalgin, A. Boogaard, I. Brunets, J. Holleman, and J. Schmitz, "Chemical modeling of a high-density inductively-coupled plasma reactor containing silane," Surface and Coatings Technology, vol. 201 (22, 23), pp. 8849-8853, 2007.

[9] I. Brunets, A.A.I. Aarnink, A. Boogaard, A.Y. Kovalgin, R.A.M. Wolters, J. Holleman, and J. Schmitz, "Low-temperature LPCVD of Si nanocrystals from disilane and trisilane (Silcore $R$ ) embedded in ALD-alumina for nonvolatile memory devices," Surface and Coatings Technology, vol. 201 (22, 23), pp. 9209-9214, 2007.

[10] A. Boogaard, A.Y. Kovalgin, I. Brunets, J. Holleman, and J. Schmitz, “Optical and Electrical Characterization of $\mathrm{SiO}_{2}$ films deposited at low temperature by means of remote ICPECVD “ Proceedings of the 10th Annual Workshop on Semiconductor Advances for Future Electronics and Sensors, pp. 404-407, 2007.

[11] A. Boogaard, A.Y. Kovalgin, I. Brunets, A.A.I. Aarnink, J. Holleman, R.A.M. Wolters, and J. Schmitz, "Characterization of $\mathrm{SiO}_{2}$ films deposited at low temperature by means of remote ICPECVD," Surface and Coatings Technology, vol. 201 (22, 23), pp. 8976-8980, 2007.

[12] A. Boogaard, A.Y. Kovalgin, I. Brunets, A.A.I. Aarnink, J. Holleman, R.A.M. Wolters, and J. Schmitz, "On the Verification of EEDFs in Plasmas with Silane using Optical Emission Spectroscopy," ECS Transactions, vol. 6 (1), pp. 259-270, 2007.

[13] A. Boogaard, A.Y. Kovalgin, A.A.I. Aarnink, R.A.M. Wolters, J. Holleman, I. Brunets, and J. Schmitz, "Langmuir-probe Characterization of an Inductively-Coupled Remote Plasma System intended for CVD and ALD," ECS Transactions, vol. 2 (7), pp. 181-191, 2007.

[14] I. Brunets, J. Holleman, A.Y. Kovalgin, T. Aarnink, A. Boogaard, P. Oesterlin, and J. Schmitz, "Green Laser Crystallization of a-Si Films Using Preformed a-Si Lines," ECS Transactions, vol. 3 (8), pp. 185-191, 2006.

[15] J. van Beek, M. van Delden, A. Jansman, A. Boogaard, A. Kemmeren, N. Pulsford, and A. den Dekker, "The integration of RF passives using thinfilm technology on high-ohmic Si in combination with thick-film interconnect," in International Symposium on Microelectronics, 9-11 Oct. 2001, Baltimore, MD, USA, 2001, pp. 467-470.

[16] A. Boogaard and J.J. van den Broek, “Crystallisation and electrical resis- 
tivity of sputter-deposited aluminium-germanium alloy films," Thin Solid Films, vol. 401 (1, 2), pp. 1-6, 2001.

[17] J.J. Van den Broek, A. Boogaard, and M. Verheijen, "Resistance switching and downtrim resistors," in 14th European Passive Components Symposium Brussels, Belgium, 2000.

[18] N.J. Pulsford, J.T.M. Van Beek, M.H.W.M. Van Delden, A. Boogaard, and R.F. Milsom, "Passive integration on Si for RF circuits in wireless applications," in Microwave Symposium Digest, 1999 IEEE MTT-S International, 1999, pp. 1897-1900.

[19] R.F. Milsom, N.J. Pulsford, A. Boogaard, and M. de Samber, “EM simulation of MCM-D implementation of PA with integrated passive components," in Radio and Wireless Conference, 1999. RAWCON 1999, pp. 255-258. 


\section{US patents}

[1] US Patent 6,251,171, Air cleaner (2001).

[2] US Patent 6,509,650, Electronic device, and method of patterning a first layer (2003).

[3] US Patent 6,529,116, Passive component (2003).

[4] US Patent 6,764,953, Electronic device, and method of patterning a first layer (2004).

[5] US Patent 7,184,122, Lithographic apparatus and device manufacturing method (2007).

[6] US Patent 7,450,217, Exposure apparatus, coatings for exposure apparatus, lithographic apparatus, device manufacturing method, and device manufactured thereby (2008).

[7] US Patent 7,557,901, Lithographic apparatus and device manufacturing method (2009).

[8] US Patent 7,746,375, Digital camera with panorama or mosaic functionality (2010). 
Appendices 


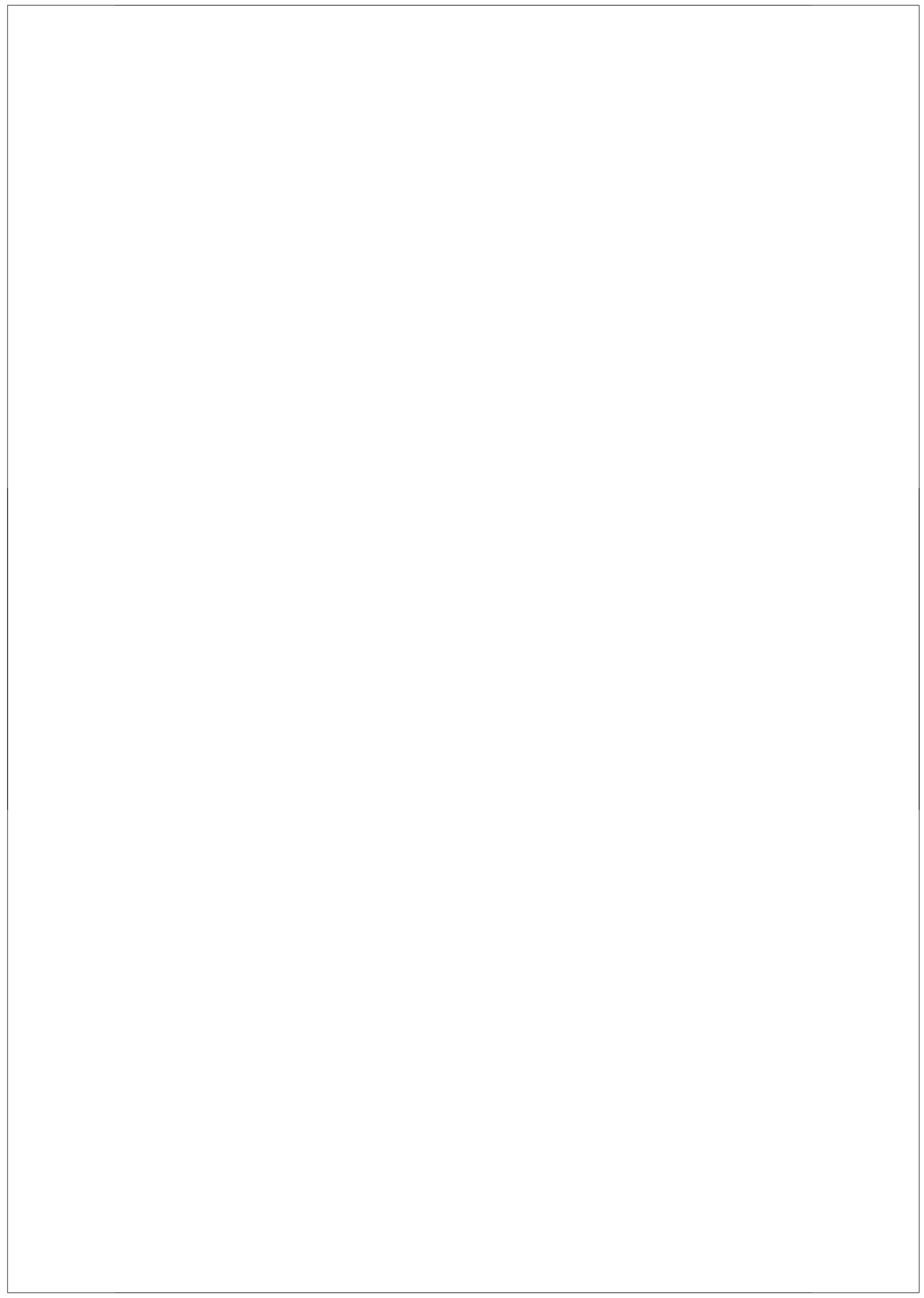




\section{Calculation of line intensity ratios}

Emission line intensity ratios were calculated according to the guide lines sketched in section 4.3. In brief, emission line intensities of ionized argon $\mathrm{Ar}^{+*}$ $\left(I_{1-2}\right.$ in Table 5.2) are divided over emission line intensities of excited argon $\operatorname{Ar}^{*}\left(I_{3-5}\right.$, see Table 5.2 for details). Two-step excitation has to be accounted for when an ion line intensity $I_{1-2}$ is calculated, while emissions $I_{3-5}$ from $\mathrm{Ar}^{*}$ can be described by one-step excitation only (please note that this is not a general rule, but a consequence of choosing the proper transition lines $I_{3-5}$, as explained in section 4.3.3).

It was derived in section 4.3.2 that the line intensity, $I_{i j}$, for a particular $i \rightarrow j$ emission line, incident on the detector is equal to

$$
I_{i j}=C h v_{i j} N_{0} N_{\mathrm{e}} \sqrt{\frac{2}{m_{\mathrm{e}}}} \int \sigma_{i j}^{\mathrm{Opt}}(E) \sqrt{E} f_{\mathrm{E}}(E) \mathrm{d} E,
$$

where $C$ is the sensitivity of the optical system at the observed wavelength, $h v_{i j}$ is the energy of transition $i \rightarrow j\left(h v_{1}=2.58 \mathrm{eV}\right.$ and $h v_{4}=2.75 \mathrm{eV}$ for $I_{1}$ and $I_{4}$, respectively), $N_{0}$ is the density of ground state atoms, $N_{\mathrm{e}}$ is the electron density, $m_{e}$ is the electron mass, $\sigma_{i j}^{\text {Opt }}$ is the $i \rightarrow j$ optical emission cross section, and $f_{\mathrm{E}}(E)$ is the electron energy distribution function.

This equation is appropriate to calculate emission lines of excited argon $\mathrm{Ar}^{*} I_{3-5}$ (see section 4.3.3):

$$
I_{3-5}=C h v_{3-5} N_{0} N_{\mathrm{e}} \sqrt{\frac{2}{m_{\mathrm{e}}}} \int \sigma_{3-5}^{\mathrm{Opt}}(E) \sqrt{E} f_{\mathrm{E}}(E) \mathrm{d} E .
$$

The modeled signal for the ion lines however should include the two- 


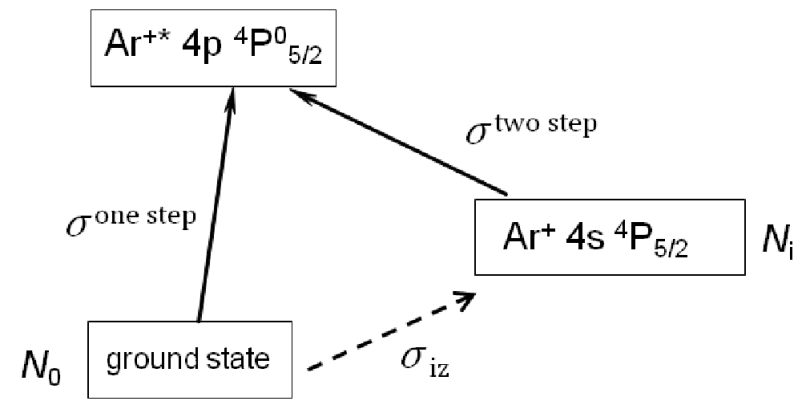

Figure A.1: Schematic of processes that populate the $\mathrm{Ar}^{+*} 4 \mathrm{p}^{4} \mathrm{P}_{5 / 2}^{0}$ level.

step contribution,

$$
I_{1-2}=I_{\mathrm{Ar}^{+}}^{\text {one step }}+I_{\mathrm{Ar}^{+}}^{\text {two step }},
$$

where $I_{1-2}$ is the calculated line intensity, $I_{\mathrm{Ar}}^{\text {one step }}$ and $I_{\mathrm{Ar}}^{+}{ }^{\text {two step }}$ are the calculated intensities using the one-step and two-step excitation mechanisms, respectively. The two excitations mechanisms needed to calculate $I_{1}$ are sketched in Figure A.1.

This leads to

$$
\begin{aligned}
I_{1-2}=\operatorname{Ch}_{1-2} N_{0} & N_{\mathrm{e}} \sqrt{\frac{2}{m_{\mathrm{e}}}} \int \sigma_{1-2}^{\text {one step }}(E) \sqrt{E} f_{\mathrm{E}}(E) \mathrm{d} E \\
& +\operatorname{Ch}_{1-2} N_{\mathrm{i}} N_{\mathrm{e}} \sqrt{\frac{2}{m_{\mathrm{e}}}} \int \sigma_{1-2}^{\text {two step }}(E) \sqrt{E} f_{\mathrm{E}}(E) \mathrm{d} E .
\end{aligned}
$$

Equation (3.1) in section 3 can be simplified to $\alpha_{\mathrm{iz}} \cong N_{\mathrm{i}} / N_{0}$ for low degrees of ionization. Inserting this into the above equation yields

$$
\begin{aligned}
I_{1-2}=C h v_{1-2} N_{0} N_{\mathrm{e}} \sqrt{\frac{2}{m_{e}}}\left(\int \sigma_{1-2}^{\text {one step }}(E) \sqrt{E} f_{\mathrm{E}}(E) \mathrm{d} E\right. \\
\left.\quad+\alpha_{\mathrm{iz}} \int \sigma_{1-2}^{\mathrm{two} \text { step }}(E) \sqrt{E} f_{\mathrm{E}}(E) \mathrm{d} E\right) .
\end{aligned}
$$

The ratio is given by

$$
\frac{I_{1-2}}{I_{3-5}}=\frac{h \nu_{1-2}\left[\int \sigma_{1-2}^{\text {one step }}(E) \sqrt{E} f_{\mathrm{E}}(E) \mathrm{d} E+\alpha_{\mathrm{iz}} \int \sigma_{1-2}^{\text {two step }}(E) \sqrt{E} f_{\mathrm{E}}(E) \mathrm{d} E\right]}{h \nu_{3-5} \int \sigma_{3-5}^{\mathrm{Opt}}(E) \sqrt{E} f_{\mathrm{E}}(E) \mathrm{d} E},
$$


assuming that the sensitivity of the optical system $C$ has the same value for both transitions.

The ionization degree $\alpha_{\mathrm{iz}}$ in equation (A.6) was calculated from the LP measurements by combining the simplified equation (3.1) with equation (4.19), and assuming $N_{\mathrm{i}} \approx N_{\mathrm{e}}$ :

$$
\alpha_{\mathrm{iz}} \cong \frac{N_{\mathrm{i}}}{N_{0}} \cong \frac{N_{\mathrm{e}}}{N_{0}} \cong \frac{\int_{0}^{\infty} N_{\mathrm{e}} f_{\mathrm{E}}(E) \mathrm{d} \varepsilon}{N_{0}} .
$$

The use of equation (A.6) is explained by showing a typical example, $I_{1} / I_{4}$. The ratio $I_{1} / I_{4}$ is calculated by

$$
\frac{I_{1}}{I_{4}}=\frac{2.58 \mathrm{eV}\left[\int \sigma_{480.6 \mathrm{~nm}}^{\text {one step }}(E) \sqrt{E} f_{\mathrm{E}}(E) \mathrm{d} E+\alpha_{\mathrm{iz}} \int \sigma_{480.6 \mathrm{~nm}}^{\mathrm{two} \text { step }}(E) \sqrt{E} f_{\mathrm{E}}(E) \mathrm{d} E\right]}{2.75 \mathrm{eV} \int \sigma_{451.1 \mathrm{~nm}}^{\mathrm{Opt}}(E) \sqrt{E} f_{\mathrm{E}}(E) \mathrm{d} E},
$$

where $f_{\mathrm{E}}(E)$ can be either the entire experimental EEDF or the MB-part only (see Figure A.2); $\alpha_{\mathrm{iz}}=2 \cdot 10^{-04}$, and the appropriate cross sections can be found in Figure 5.6 and in $[108,119,149]$. These cross sections sometimes have large energy intervals between the data points. Analytical functions were interpolated to increase the accuracy [149].

Several calculation steps can be found in Figure A.2 and in Table A.1. The first step is to calculate the excitation rates, $\int \sigma(E) \sqrt{E} f_{\mathrm{E}}(E) \mathrm{d} E$, for the reactions under consideration, i.e., one-step and two-step excitation of $\mathrm{Ar}^{+}$and ground state excitation of Ar. The partial excitation rates, $\sigma(E) \sqrt{E} f_{\mathrm{E}}(E) \mathrm{d} E$, are calculated by multiplying the values of the cross section and $\sqrt{E} f_{\mathrm{E}}$ in a specific energy interval $d E$, see Figure A.2. The numerical integrals obtained from Figure A.2 are shown in Table A.1. The next step is to multiply the excitation rates of the two-step processes by the ionization degree, $\alpha_{\mathrm{iz}}$, see equation (A.8) and Table A.1. Intensity value are obtained if we multiply the excitation rates by the energy per photon: $h v_{i j}$, see Table A.1.

Some observations concerning the fast-electron tail can be made from Figure A.2 and Table A.1. First, rates are 2-4 orders of magnitude larger for those calculated by the entire EEDF than those calculated by MB-part only. Clearly, this is due to the presence of the fast-electron tail of the measured EEDF. Second, line intensities are completely determined by two-step excitation for MB-EEDFs, whereas one-step excitation is the dominant process when the fast electrons are considered as well. 

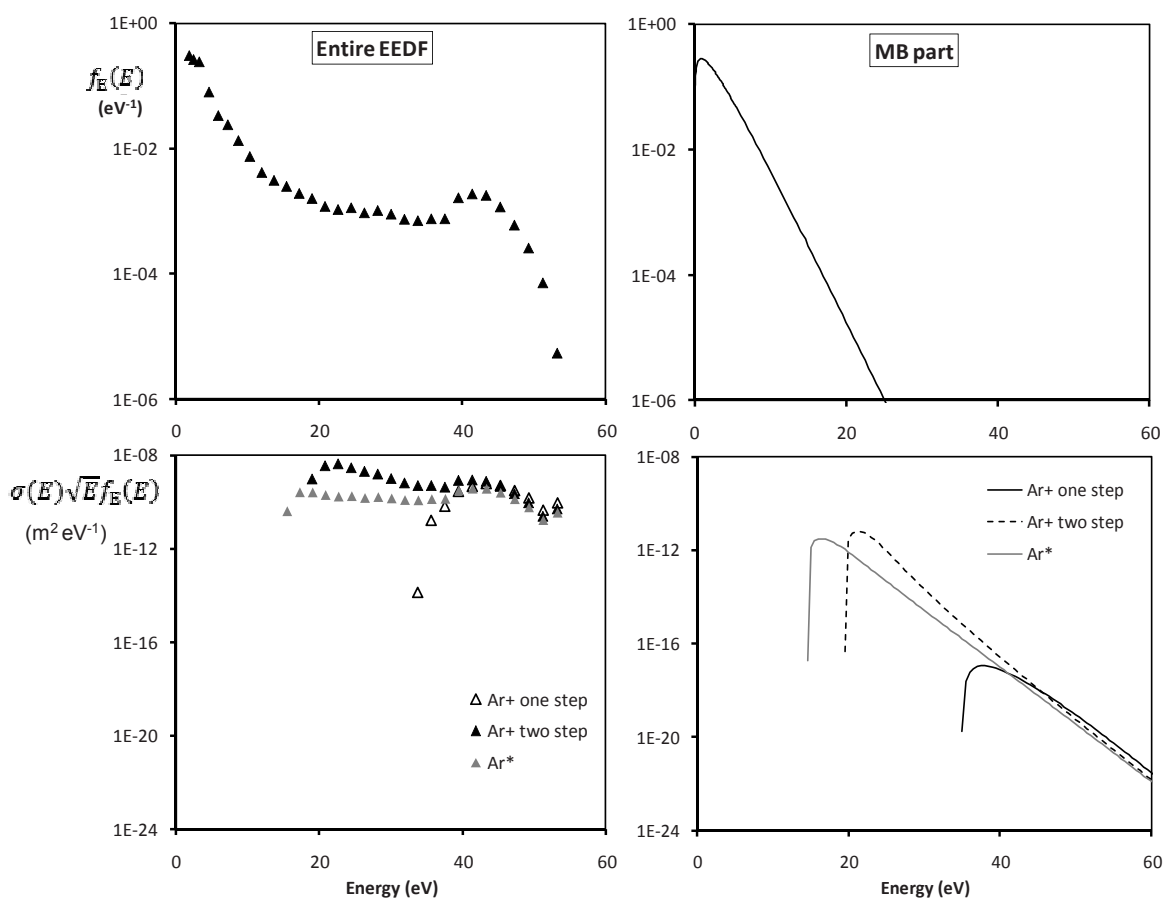

Figure A.2: EEDF and partial excitation rates, $\sigma(E) \cdot \sqrt{E} \cdot f_{E}(E) E$, for argon plasma at $1 \mathrm{~Pa}$. EEDF was taken at $240 \mathrm{~mm}$ (see Figure 4.1). Cross sections were taken from $[108,119,149]$. 


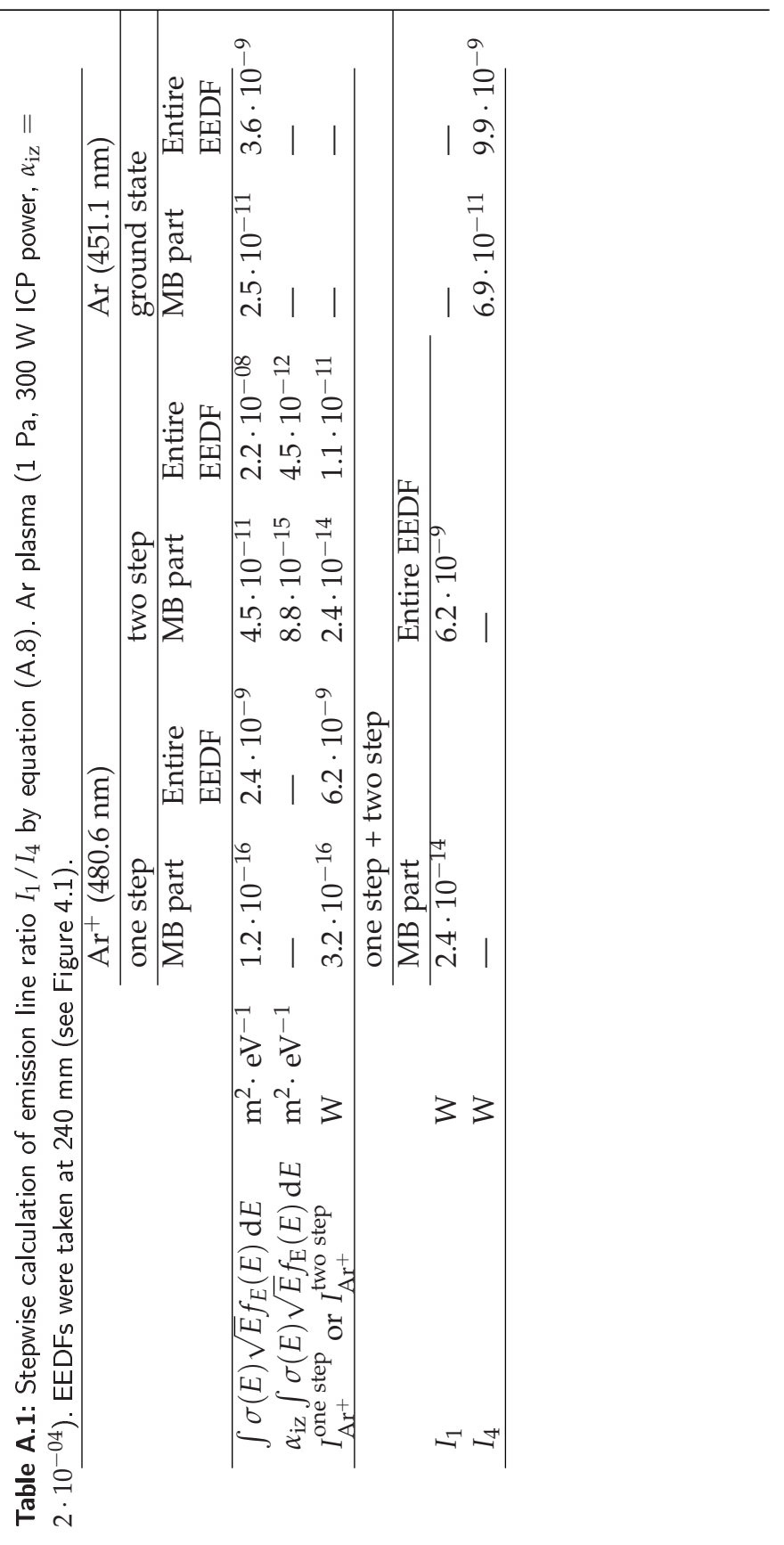




\section{Line intensities caused by $\operatorname{Ar} 1 \mathrm{~s}_{5}$-metastables}

The density of metastables can be estimated by the $I_{\mathrm{Ar}^{+}} / I_{\mathrm{Ar}}$ line ratio method as described in Appendix A. The analysis should then include emission of $2 p_{9}$ level (811.5-nm emission line, $I_{6}$, see Table 5.2) since this level is partly populated from the $1 \mathrm{~s}_{5}$ metastable level, see Figure B.1.

The derivation follows the one in Appendix A, but both levels (i.e., $I_{1-2}$ and $\left.I_{6}\right)$ should include two-step excitation. The line intensity $I_{6}$ can be de-

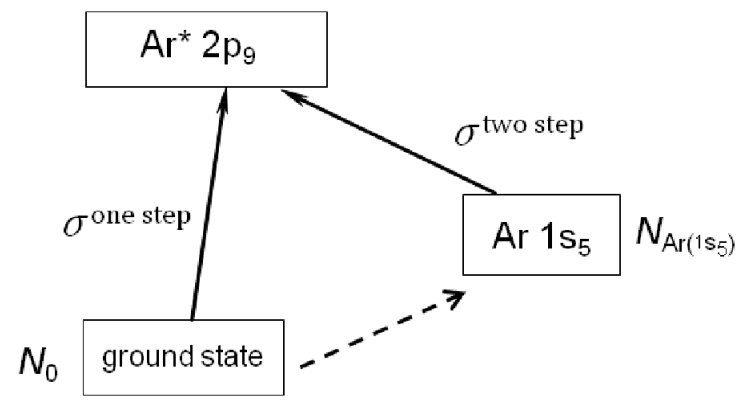

Figure B.1: Schematic of processes that populate the $\mathrm{Ar}^{*} 2 \mathrm{p}_{9}$ level. 
scribed similarly to equation (A.5) of the $\mathrm{Ar}^{+*}$ level:

$$
\begin{aligned}
I_{6}=C h v_{6} N_{0} N_{\mathrm{e}} \sqrt{\frac{2}{m_{e}}}\left(\int \sigma_{6}^{\text {one step }}\right. & (E) \sqrt{E} f_{\mathrm{E}}(E) \mathrm{d} E \\
& \left.+\alpha_{\mathrm{ms}} \int \sigma_{6}^{\mathrm{two} \mathrm{step}}(E) \sqrt{E} f_{\mathrm{E}}(E) \mathrm{d} E\right),
\end{aligned}
$$

where $I_{6}$ is the calculated line intensity, $\sigma_{6}^{\text {one step }}$ is the ground-state excitation cross section, and $\sigma_{6}^{\text {two step }}$ is the metastable-state excitation cross section to the same level (see Table 5.2 and Figure B.1). $\alpha_{\mathrm{ms}}$ is defined as the ratio of the number of metastables to the number of ground state atoms, i.e., $\alpha_{\mathrm{ms}}=N_{\mathrm{Ar}\left(1 \mathrm{~s}_{5}\right)} / N_{0}$.

The line intensity ratio is given by

$$
\frac{I_{1-2}}{I_{6}}=\frac{h v_{1-2}\left[\int \sigma_{1-2}^{\text {one step }}(E) \sqrt{E} f_{\mathrm{E}}(E) \mathrm{d} E+\alpha_{\mathrm{iz}} \int \sigma_{1-2}^{\text {two step }}(E) \sqrt{E} f_{\mathrm{E}}(E) \mathrm{d} E\right]}{h v_{6}\left[\int \sigma_{6}^{\text {one step }}(E) \sqrt{E} f_{\mathrm{E}}(E) \mathrm{d} E+\alpha_{\mathrm{ms}} \int \sigma_{6}^{\text {two step }}(E) \sqrt{E} f_{\mathrm{E}}(E) \mathrm{d} E\right]}
$$

The ratio $I_{1-2} / I_{6}$ can be fitted to the measured ratio by adjusting $\alpha_{\mathrm{ms}}$, since $f_{\mathrm{E}}(E)$ and $\alpha_{\mathrm{iz}}$ can be measured, and the appropriate cross sections are known (see Figure 5.6). Finally, $N_{\mathrm{Ar}\left(1 \mathrm{~s}_{5}\right)}$ can be calculated from the obtained $\alpha_{\mathrm{ms}}$.

Using equation (B.2) yielded $\alpha_{\mathrm{ms}}=1.7 \cdot 10^{-5}$ for Ar plasma at $1 \mathrm{~Pa}$ (see Figure B.2 for the EEDF and the calculated excitation rates; $h v_{1}=2.58 \mathrm{eV}$, $\left.h v_{6}=1.53 \mathrm{eV}, \alpha_{\mathrm{iz}}=2.1 \cdot 10^{-4}\right)$. Therefore, $N_{\mathrm{Ar}\left(1 \mathrm{~s}_{5}\right)}$ was $4 \cdot 10^{15} \mathrm{~m}^{-3}$.

Figure 5.10 showed that the electron tail was not significantly affected by the addition of $\mathrm{SiH}_{4}$ below 8 vol.\%. It was calculated by Pfau [150] that the number of slow electrons increases when one adds silane to argon plasmas, and this effect is missed by regular OES methods. At this point, the reader should recall that these slow electrons do affect the emission of the Ar 811.5 $\mathrm{nm}$ line, $I_{6}$, because this level is also populated by $1 s_{5}$-metastables ( $c f$. open circles in Figure B.2). Thus, an analysis of the entire EEDF (including the MBpart) should include emission line $I_{6}$.

To estimate the influence of $\mathrm{SiH}_{4}$ additions on emission line $I_{6}$, we split our entire-range experimental EEDF (see upper part of Figure B.2) in two parts, (i) a Maxwellian part, and (ii) an electron tail. These two distributions are assigned with a different electron density, i.e., $N_{\mathrm{e}}^{\mathrm{MB}}$ to the MB-part, and $N_{\mathrm{e}}-$ the original, undisturbed, electron density — to the high-energy tail. The line 

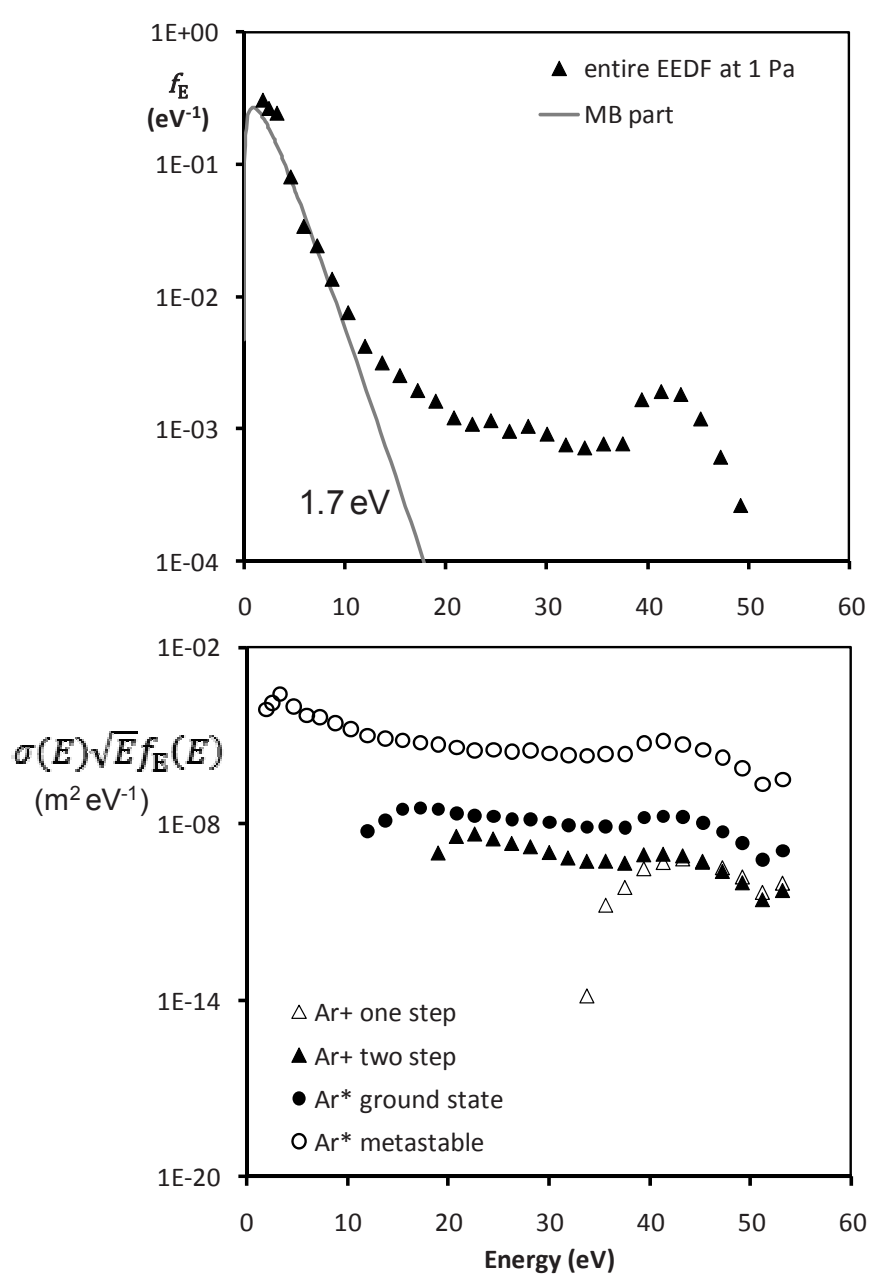

Figure B.2: EEDF and partial excitation rates, $\sigma(E) \cdot \sqrt{E} \cdot f_{E}(E) E$, for argon plasma at $1 \mathrm{~Pa}$. EEDF was taken at $240 \mathrm{~mm}$ (see Figure 4.1). Cross sections were taken from $[113,119,149]$. 
intensity of a transition is then given by

$$
\begin{aligned}
I_{i j}=C h v_{i j} N_{0}\left[N_{\mathrm{e}}^{\mathrm{MB}}\left(k T_{\mathrm{e}}\right)^{-3 / 2}\right. & \int_{0 \mathrm{eV}}^{12 \mathrm{eV}} \sigma_{i j}^{\mathrm{Opt}}(E) \exp \left(\frac{-E}{k T_{\mathrm{e}}}\right) E \mathrm{~d} E \\
& \left.+N_{\mathrm{e}} \sqrt{\frac{2}{m_{\mathrm{e}}}} \int_{12 \mathrm{eV}}^{\infty} \sigma_{i j}^{\mathrm{Opt}}(E) \sqrt{E} f_{\mathrm{E}}(E) \mathrm{d} E\right]
\end{aligned}
$$

By equation (B.3), it is possible to calculate emission line intensities for situations where silane addition will influence the density of slow electrons (i.e., $N_{\mathrm{e}}^{\mathrm{MB}}$ will be varied), while the fast-electron tail can be kept constant (hence $N_{\mathrm{e}}=$ constant).

In principle, equation (B.3) could be used for every excitation process under investigation (in our case: $I_{1-6}$ ). However, the optical cross sections of most excitation processes ( $I_{1-5}$ in this study) are zero in the interval between 0 and $12 \mathrm{eV}$, and hence varying $N_{\mathrm{e}}^{\mathrm{MB}}$ does not influence their intensities. This mimics exactly what we measured in Figure 5.10, i.e., line intensities were not affected by the addition of $\mathrm{SiH}_{4}$ below 8 vol.\% because the electron tail remained constant. However, varying $N_{\mathrm{e}}^{\mathrm{MB}}$ does influence $I_{6}$ of the $\operatorname{Ar}\left(2 \mathrm{p}_{9}\right)$ level since it is partly populated via the $\operatorname{Ar}\left(1 \mathrm{~s}_{5}\right)$ metastable state. One of its cross sections, $\sigma_{6}^{\text {two step }}$, is larger than zero in this interval, and the intensity caused by this transition has to be described by equation (B.3). Inserting equation (B.3) in equation (B.1) yields

$$
\begin{aligned}
& I_{6}=C h v_{6} N_{0}\left\{\sqrt{\frac{2}{m_{e}}} N_{\mathrm{e}} \int_{0 \mathrm{eV}}^{\infty} \sigma_{6}^{\text {one step }}(E) \sqrt{E} f_{\mathrm{E}}(E) \mathrm{d} E\right. \\
& +\alpha_{\mathrm{ms}}\left[N_{\mathrm{e}}^{\mathrm{MB}}\left(k T_{\mathrm{e}}\right)^{-3 / 2} \int_{0 \mathrm{eV}}^{12 \mathrm{eV}} \sigma_{6}^{\text {two step }}(E) \exp \left(\frac{-E}{k T_{\mathrm{e}}}\right) E \mathrm{~d} E\right. \\
& \left.\left.+N_{\mathrm{e}} \sqrt{\frac{2}{m_{\mathrm{e}}}} \int_{12 \mathrm{eV}}^{\infty} \sigma_{6}^{\text {two step }}(E) \sqrt{E} f_{\mathrm{E}}(E) \mathrm{d} E\right]\right\} .
\end{aligned}
$$

We will now estimate the influence of $\mathrm{SiH}_{4}$ additions on emission line $\mathrm{I}_{6}$ for the particular case depicted in Figure B.2 (i.e., Ar plasma at $1 \mathrm{~Pa} ; h v_{6}=1.53$ $\mathrm{eV}, N_{0}=2.4 \cdot 10^{20} \mathrm{~m}^{-3}, N_{\mathrm{Ar}\left(1 \mathrm{~s}_{5}\right)}=4 \cdot 10^{15} \mathrm{~m}^{-3}, \alpha_{\mathrm{ms}}=1.7 \cdot 10^{-5}, N_{\mathrm{e}}=5 \cdot 10^{16}$ $\mathrm{m}^{-3}, \alpha_{\mathrm{iz}}=2.1 \cdot 10^{-4}$, the MB part is characterized by a $k T_{\mathrm{e}}$ of $\left.1.7 \mathrm{eV}\right)$. Pfau [150] calculated (based on MB-statistics and collision rate data) a two-times higher density of slow MB-electrons in a $\mathrm{Ar}-5 \% \mathrm{SiH}_{4}$ plasma compared to a pure argon plasma (i.e., $N_{\mathrm{e}}^{\mathrm{MB}}=2 N_{\mathrm{e}}$ ). Inserting this $N_{\mathrm{e}}^{\mathrm{MB}}$ into the calculation of $I_{6}$ (by equation (B.4)), would increase its intensity by about $20 \%$. One can estimate that the addition of $2 \mathrm{vol} . \%$ of silane to argon plasma would increase $I_{6}$ by only 
$8 \%$. This increase was not measurable since the experimental error of the OES measurements was already $40 \%$. To be on the safe side, 0.8 vol. $\%$ was used as the highest silane fraction in this thesis. It increases the experimental error by only $3 \%$. Hence, the original full-range experimental EEDF obtained without silane was considered as valid for the silane-containing plasma as well.

N.B. Gas-phase reactions can also be modeled at silane fractions between 0.8 and 8 vol.\% by adjusting the MB-part of the experimentally measured EEDF to the desired silane fraction, as described above. This adjusted EEDF could be used to calculate the electron-stimulated reactions in $\left(\mathrm{Ar}-\mathrm{N}_{2} \mathrm{O}-\mathrm{SiH}_{4}\right)$ plasmas. 
"Hoe sprong mijn hart hoog op in mij

toen Rob mij zeide: 'gord $u$ aan,

om naar het Mesa+ te gaan,

kom ga met ons en doe als wij'."

En zo mocht ik, voor het krieken van de dag, een keertje met Rob Wolters mee op zijn wekelijkse reis naar Enschede. Rob introduceerde mij daar met ongeveer deze woorden: "Ik breng jullie deze knaap. Hij kan al wat, maar heeft nog wel slaag nodig." Vervolgens werd ik genadiglijk aangenomen als promovendus.

$\mathrm{Nu}$ ik in het kader van dit proefschrift de gelegenheid krijg een dankwoord uit te brengen is dit de plaats om mensen te bedanken die in het heden en verleden invloed hebben gehad op mijn loopbaan en dus (indirect) ook op dit proefschrift.

En dit zijn de namen van hen aan wie ik dierbare herinneringen heb:

- Onderzoekers op het Nat.Lab. die rechtstreeks invloed hebben gehad op mijn ontwikkeling: Joost van Beek, Jan van den Broek, Falco van Delft, Johan Marra, Richard van der Rijt, Marc de Samber, Rob Wolters.

- Promotor Rob Wolters en co-promotor Alexey Kovalgin. Ze legden de breidel op mijn schrijfsel. Zij waren uitstekende reisgenoten, zowel letterlijk als figuurlijk.

- Jurriaan Schmitz dank ik voor het vertrouwen. Terwijl iedereen dacht "Wanneer gaat Arjen nu eens stoppen met plasmakoekeloeren en eindelijk eens laagjes deponeren?", bleef hij (althans naar mij toe) geduldig 
wachten op de afloop van de zaak.

- Jisk Holleman en Tom Aarnink roem ik tezamen. Zij verbaasden mij telkens met hun brede kennis van de dunnefilmtechnologie. Hun nietaflatende inzet voor studenten en promovendi zij alle mensen bekend.

- Niemand is onmisbaar, maar Annemiek Janssen is veel onmisbaarder dan anderen.

- Ihor Brunets was a great colleague. He contributed to this thesis in many ways. Thank you!

- Jos Smeets bedank ik voor het proeflezen en opmaken van de tekst, en voor het ontwerpen van de kaft.

- I thank my office mates and colleagues with whom I could share the ups and downs of doing research, or just talk about something else for a change: Radko Bankras, Boni Boksteen, Victor Blanco Carballo, Max Chefdeville, Erik Faber, Alfons Groenland, Tom van Hemert, Rodolf Herfst, Tu Hoang, Andre Hof, Ray Hueting, Gratiela Isai, Sumy Jose, Buket Kaleli, Naveed Kazmi, Jay Kolhatkar, Jiwu Lu, Phuong LeMinh, Joost Melai, Giulia Piccolo, Vidhu Puliyankot Palackavalapil, Bijoy Rajasekharan, Balaji Rangarajan, Cora Salm, Guido Sasse, Jan-Laurens van der Steen, Hao Van Bui, Sander Smits, Natalie Tiggelman. Sincere thanks to all of you.

- The author gratefully acknowledges the time and effort of the entire doctorate committee in evaluating this work.

Terwijl het nog niet eens in mij was opgekomen zette Esther Janse mij op het promotiespoor. "Wat een vrouw niet kan doen" (H. van der Lubbe).

Aan allen die (in wisselende bewoordingen) vroegen: "Wordt dat nog wat, met dat proefschrift van jou?", ligt hier mijn antwoord. Al had ik wel wat slaag nodig. 